\title{
A REALIST EVALUATION OF A RECOVERY-ORIENTED INPATIENT SERVICE.
}

Submitted to Swansea University in fulfilment of the requirements for the Degree of Doctor of Philosophy.

Amy Pritchard BSc Msc 


\section{ABSTRACT}

Background: Finding effective means of supporting individuals with a mental illness in their recovery is essential. A new recovery-oriented inpatient service in South Wales, "Woodlands", aimed to provide an environment that would support individuals with severe and enduring mental illness in their recovery. Woodlands focuses on providing a staffing group that were recovery-oriented and deliver high levels of therapeutic engagement, as well as promoting choice and responsibility to develop service-users' skills and confidence for living in the community in the future. This thesis aimed to explain which parts of Woodlands worked, for whom and in what circumstances.

Design: Three areas of Woodlands' service delivery were evaluated. This included Woodlands trying to establish itself as a new service, how the staffing model supported individuals in their recovery, and how service-user choice and responsibility was promoted and supported by the service. Realist evaluation and ecological systems theory were used to guide the analysis of multiple data strands. This included quantitative data routinely collected at Woodlands and qualitative research interviews. The qualitative research interviews were conducted with senior figures involved in the design of the service, staff members, service-users and commissioners who were involved in referring individuals to Woodlands.

Findings: The findings of this study are multifaceted and focus on the conditions of successful or unsuccessful implementation and delivery of a new recovery-oriented inpatient service. Such conditions included there being a market demand for this type of service and having the ability to quantifiably evidence the effectiveness of the service in order to secure referrals. Several service-user and staff characteristics were identified as conditions for the successful or unsuccessful engagement with key resources at Woodlands. These individual-level conditions included service-users and staff having the confidence, skills and desire to engage or deliver these resources. The congruence model (Nadler \& Tushman, 1980) was used to provide explanatory power to the findings of this thesis, focusing specifically upon Woodlands challenge of establishing itself as a provider. The findings highlight that the four facets of organisational effectiveness (the people, tasks, culture and structure), were not congruent with the service's inputs, nor were they congruent when the service was forced to adapt its service-user criterion.

Discussion: The findings are presented in a nuanced middle-range theory which uses the underpinnings of the congruence model (Nadler \& Tushman, 1980). The nuanced theory captures the challenges of establishing a new mental health service, which has translatability for other services trying to establish themselves within the competitive and commercial arena of healthcare. The findings of this thesis raise the question of whether recovery-oriented care can ever truly be achieved within the confines of inpatient care and it is argued that perhaps what services are really doing is a form of contemporary rehabilitation but dressed up in the clothes of recovery. 


\section{DECLARATIONS AND STATEMENTS}

\section{Declaration}

This work has not previously been accepted in substance for any degree and is not being concurrently submitted in candidature for any degree.

Signed__ Date_02/09/2020__

\section{Statement One}

This work is the result of my own independent study/investigation, except where otherwise stated. Other sources are acknowledged by footnotes giving explicit references. A bibliography is appended.

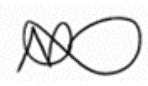

Signed Date_02/09/2020

\section{Statement Two}

I hereby give my consent for my work, if relevant and accepted, to be available for photocopying and for inter-library loan, and for the title and summary to be made available to outside organisations.

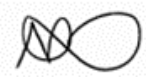

Signed

Date_02/09/2020 


\section{ACKNOWLEDGEMENTS}

Firstly, I would like to express my sincere gratitude to my supervisory team Professor Jaynie Rance and Professor Michael Coffey. Thank you for your continuous support, patience, motivation and knowledge. I am appreciative of you allowing me the freedom to learn while also keeping me on track when navigating the challenges that arose during the research. I have learnt a lot throughout the process and could not have asked for better mentors. I would also like to thank all the individuals who participated in this project for sharing their insight and experiences; and specifically, Helen Bennett for her support and guidance throughout.

I also want to extend my appreciation to my family and friends who have supported me throughout. Mum, who would have thought that the little girl who threw books at you and refused to learn would go on to dedicate three years of her life to reading, learning and writing! Thank you for never giving up on me, being my shoulder to cry on and for inspiring me to follow my dreams. Dad, who would have thought those endless conversations about me achieving my dream of becoming a Doctor would eventually come true. Thank you for always pushing me to achieve my best, for your hard work and resilience to put me in a position where success, university and this PhD were possible. David, despite claiming to be the 'most intelligent' child and the 'Uni of' digs, I think this PhD firmly places me as not only the 'most intelligent' child, but also as the 'favourite' child. Thank you for showing me that regardless of what life throws at us, I always have you by my side. Despite the ups and downs we have experienced as a family; this thesis represents that together we can achieve anything. "I am a Pritchard, I am the best, whatever I do, I do my best, whatever I do, I am the best." I would also like to thank Kuly for inspiring me to apply for the PhD and believing in me when I did not believe in myself.

Lastly, I would like thank Todd for your love, kindness and patience. Despite living hundreds of miles apart I would not have been able to achieve this without you. Our endless laughter, you coping with my tantrums when things did not go to plan with the research and being my best friend meant this thesis was completed, even when I thought it was not possible. Thank you for ensuring I look after myself physically and mentally with your endless programming so completing the $\mathrm{PhD}$, and some powerlifting competitions along the way, was possible.

I dedicate this thesis to my family and those who are no longer with us. 


\section{CONTENTS}

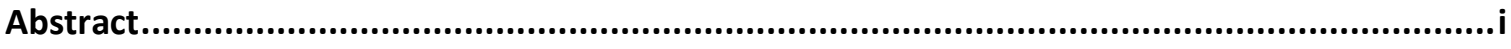

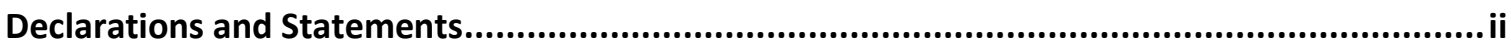

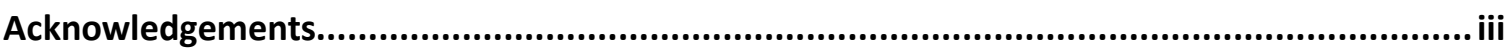

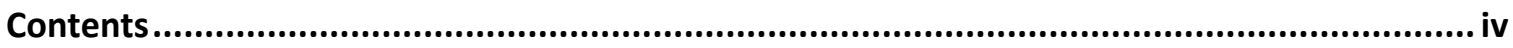

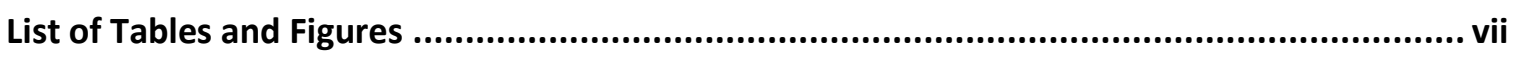

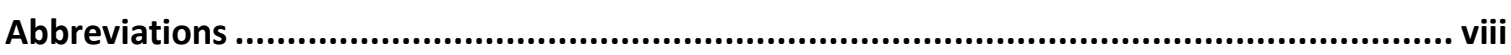

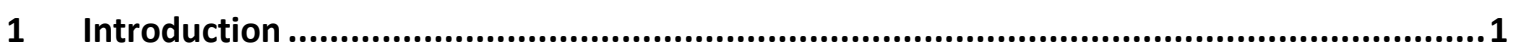

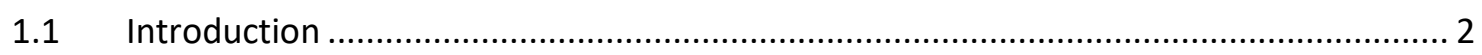

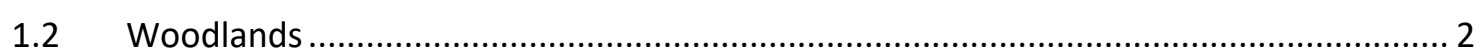

$1.3 \quad$ The Context in which Woodlands Operates ............................................................ 3

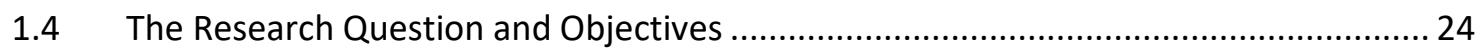

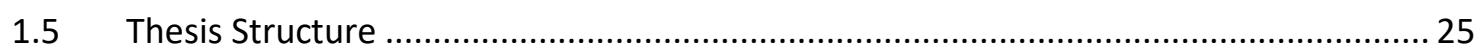

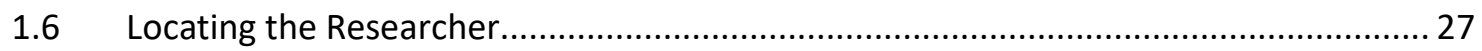

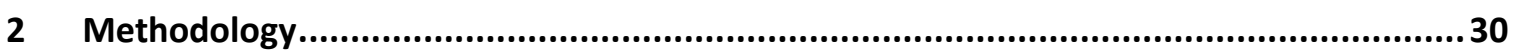

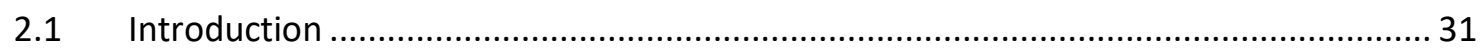

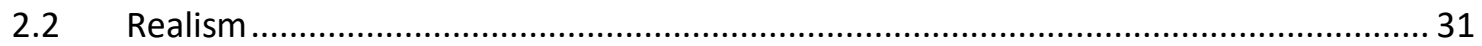

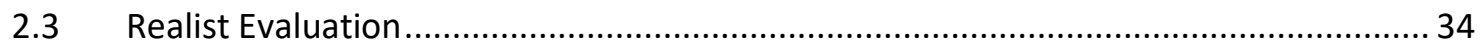

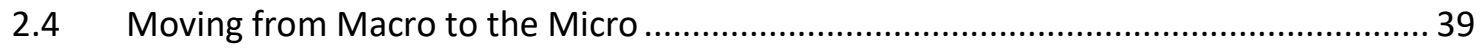

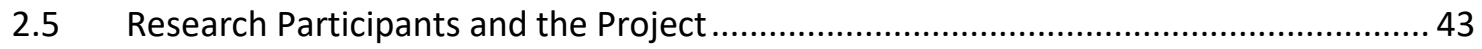

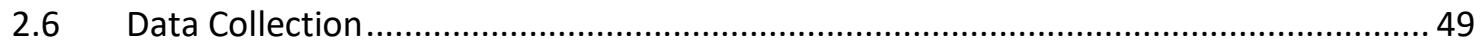

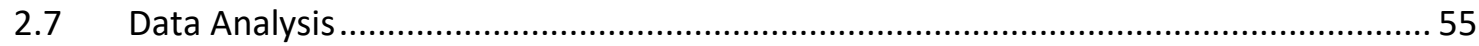

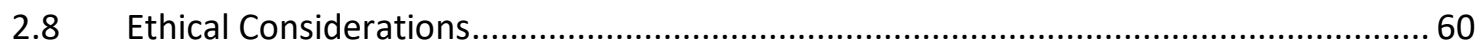

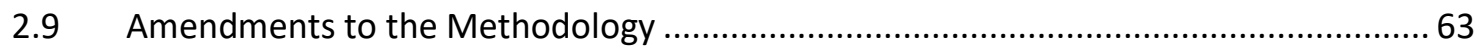

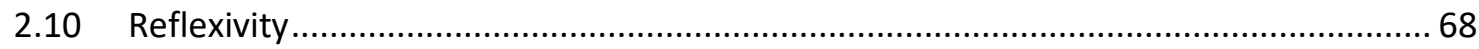

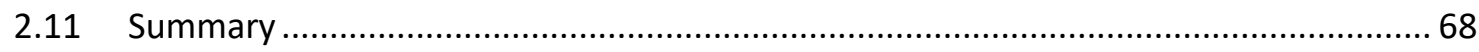

3 Recovery-Oriented Inpatient Care - Literature Review .................................................69

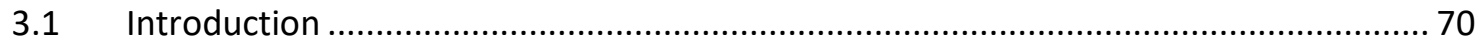

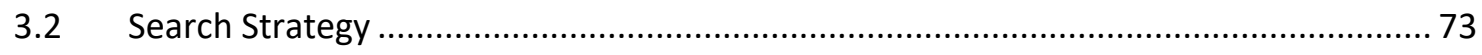

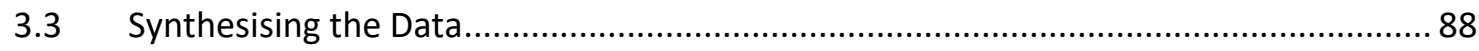

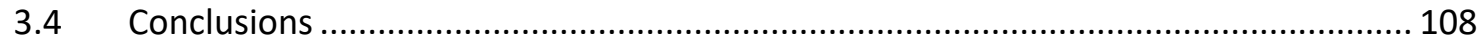

$4 \quad$ Findings - The Wider Mental Health System .............................................................. 115

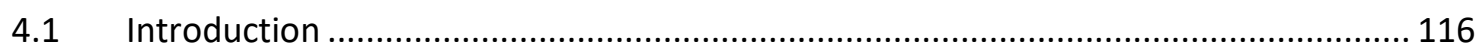

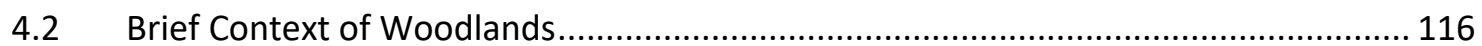


4.3 Interview Participant Details .................................................................. 117

4.4 Who was Woodlands Intended to Support? ...................................................... 120

4.5 The Troubles of Securing Referrals............................................................. 122

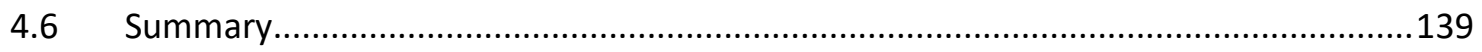

$5 \quad$ Findings - The Staffing Model: Therapeutic Engagement ..................................... 145

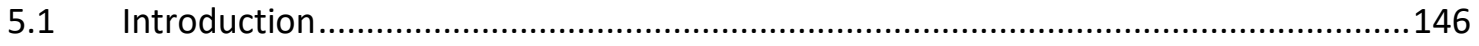

5.2 The Intended Staff Qualities .................................................................. 147

5.3 The Realities of Staff Qualities ............................................................... 149

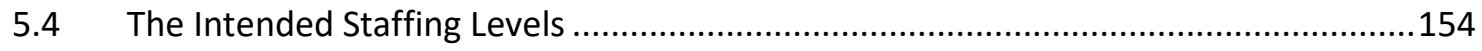

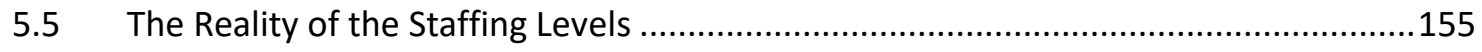

5.6 The Intention for Including Peer Mentors .................................................... 160

$5.7 \quad$ The Realities of the Role of Peer Mentors .................................................. 163

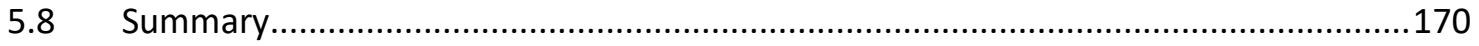

6 Findings - Service-user Choice and Responsibility......................................... 174

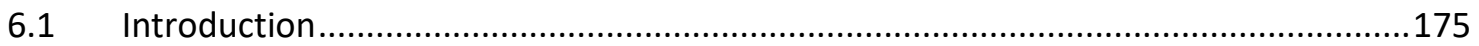

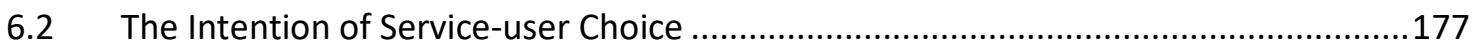

6.3 The Realities of Service-user Choice............................................................... 181

6.4 The Intention for Service-users to Take Responsibility for Daily Living Tasks.............192

6.5 The Reality of Daily Living Tasks .................................................................. 194

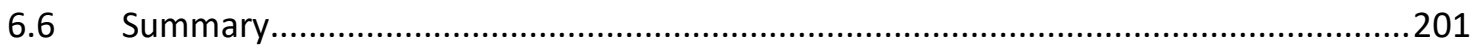

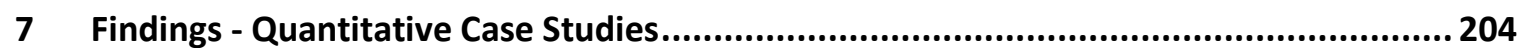

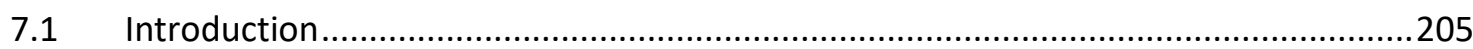

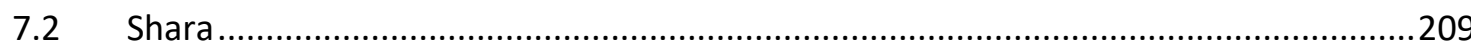

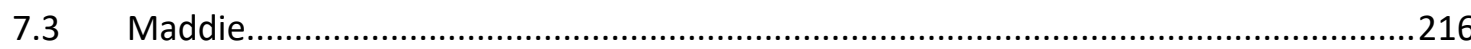

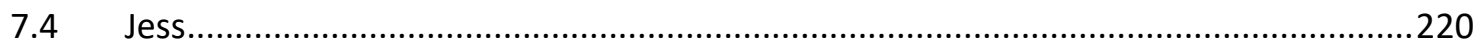

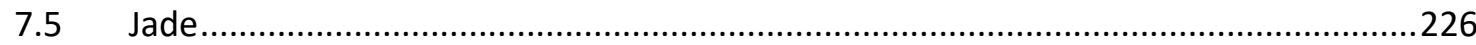

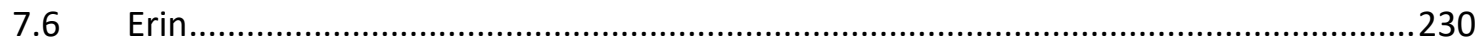

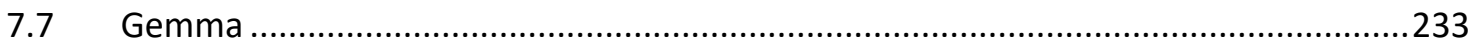

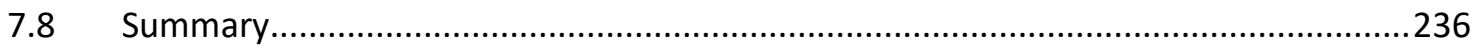

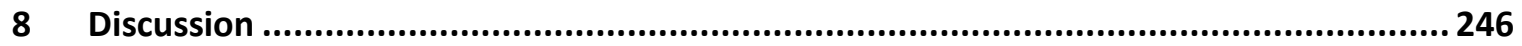

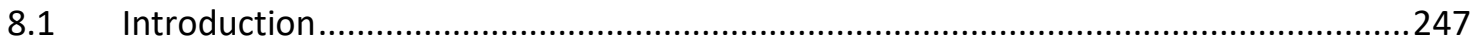

8.2 Recapitulation of the Study's Purpose .........................................................247

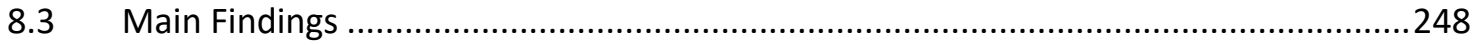

8.4 Findings in Relation to Recovery-Oriented Inpatient Care Literature.......................269

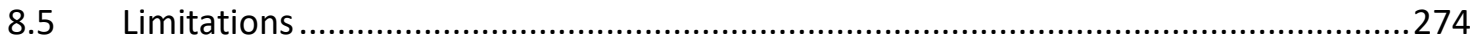

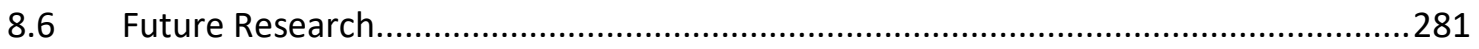




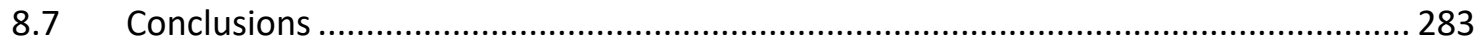

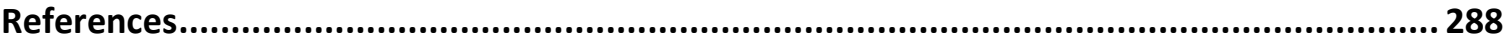

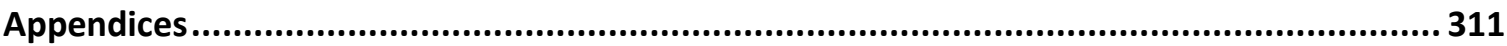




\section{LIST OF TABLES AND FIGURES}

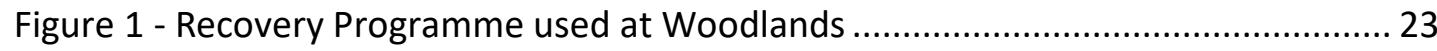

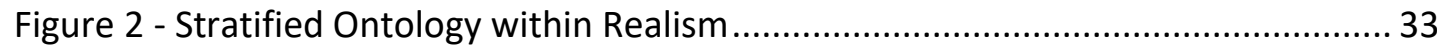

Figure 3 - The Levels of Bronfenbrenner's Ecological Systems Theory ........................... 41

Figure 4 - Adapted Framework Analysis Process (Ritchie \& Spencer, 1994)................... 57

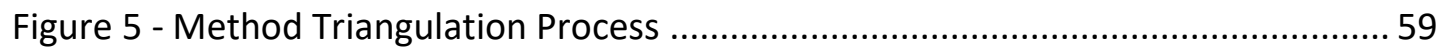

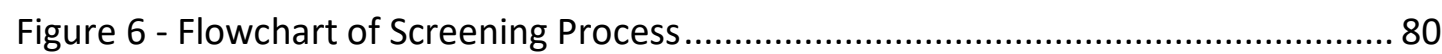

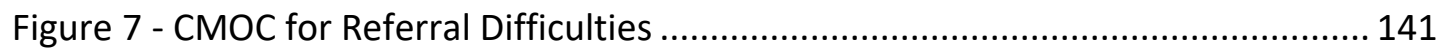

Figure 8 - Congruence Model (Nadler \& Tushman, 1980) .......................................... 144

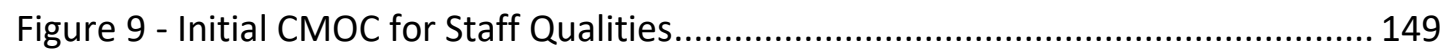

Figure 10 - Revised CMOC for Staff Qualities ............................................................. 153

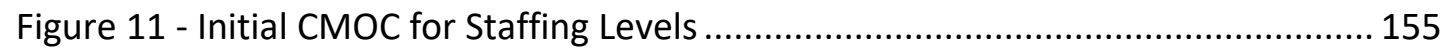

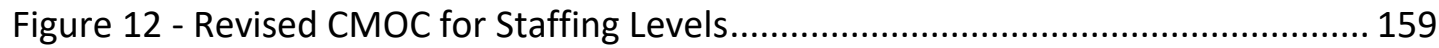

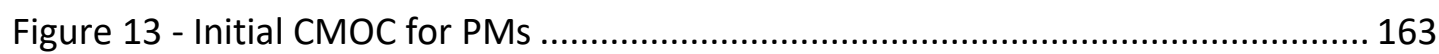

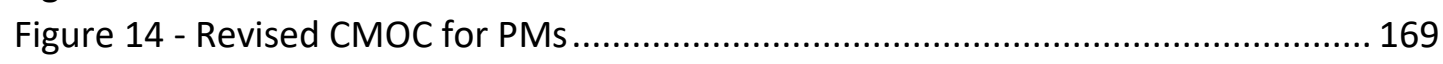

Figure 15 - Initial CMOC for Service-user Choice...................................................... 181

Figure 16 - Revised CMOC for Service-user Choice .................................................... 191

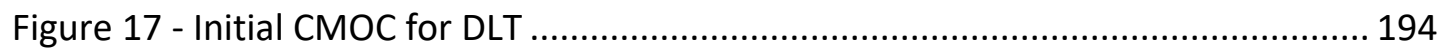

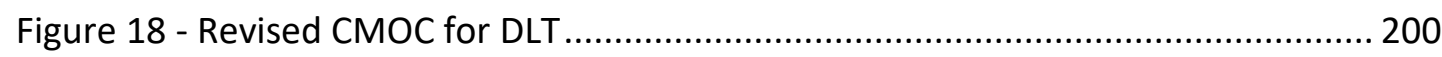

Figure 19 - Proposed Middle-Range Theory (Nasler \& Tushman, 1980) ......................... 268

Figure 20 - Using the EST to understand the contextual layers relevant to Woodlands 273

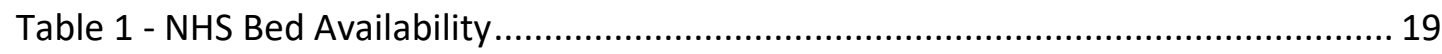

Table 2 - Independent Mental Health Hospitals in Wales ............................................. 20

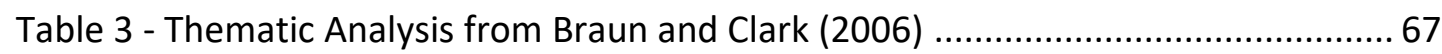

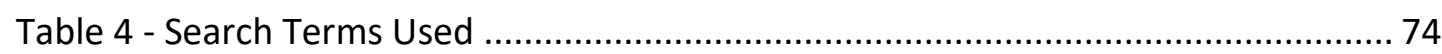

Table 5 - Papers included within the review .............................................................. 88

Table 6 - CORE-OM Scores relating to Severity of Service-User Psychological Distress 207

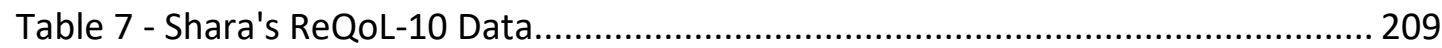

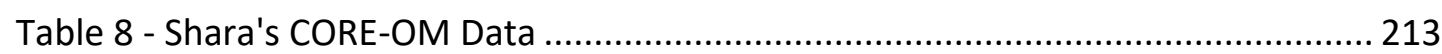

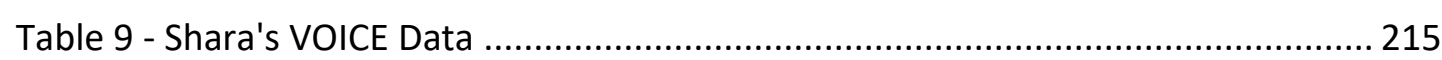

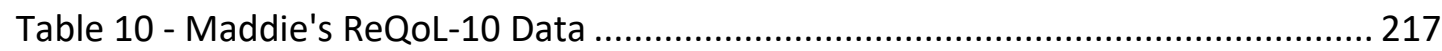

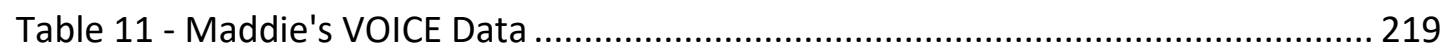

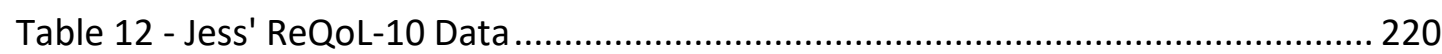

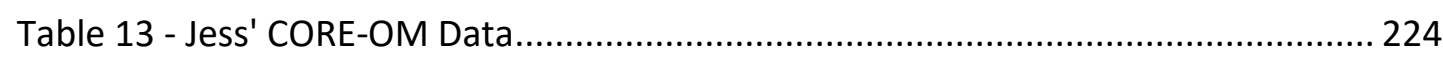

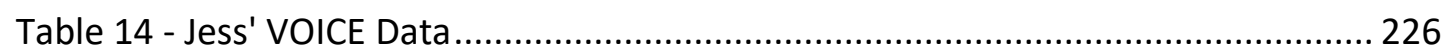

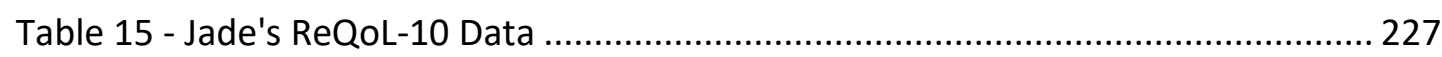

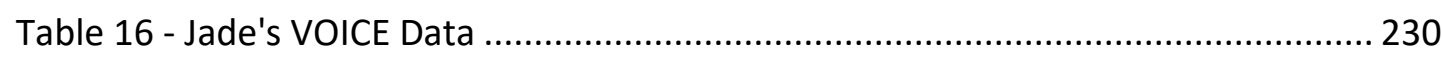

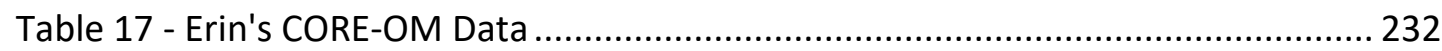

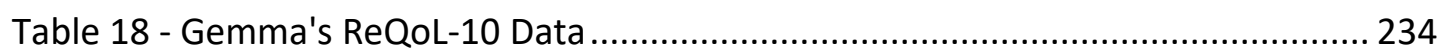

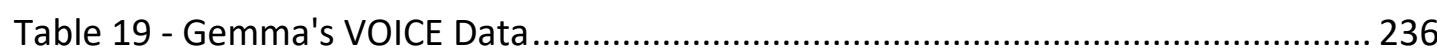




\section{ABBREVIATIONS}

ACTfP - Acceptance and Commitment Therapy Group for Psychosis

CARES - Centre for Advancement in Realist Evaluation and Synthesis

CASP - Critical Appraisal Skills Programme

CORE-OM - Clinical Outcomes in Routine Evaluation Outcome Measure

CMOC - Context-Mechanism-Outcome Configuration

DLT - Daily Living Tasks

EST - Ecological Systems Theory

GDPR - General Data Protection Regulation

LHB - Local Health Board

HRA - Health Research Authority

IPA - Interpretative Phenomenological Analysis

MCA - Mental Capacity Act

MMAT - Mixed Methods Appraisal Tool

NHS - National Health Service

PA/s - Programme Architect/s

PD - Personality Disorder

PHE - Physical Health Education

PMs - Peer mentor/s

PPCT - Process-Person-Context-Time

RE - Realist Evaluation

QoL - Quality of Life

REC - Research Ethics Committee

ReQoL-10 - Recovering Quality of Life - 10

ROC - Recovery-oriented care

UK - United Kingdom

USA - United States of America

VOICE - Views on Inpatient Care 


\section{INTRODUCTION}




\subsection{Introduction}

Woodlands is a mental health inpatient facility that opened in 2017 in South Wales, with the aim of supporting individuals with severe and enduring mental illness in their recovery. This thesis reports on an evaluation of Woodlands, which was conducted using Realist Evaluation (RE) (see section 2.3). The evaluation will address what works, for whom and in what circumstances in relation to Woodlands establishing itself as a new service within the mental health system. The evaluation will then focus upon two areas of the service's philosophy - the staffing group and service-user choice and responsibility, as well as analysis of routinely collected data from the service.

In this chapter, I briefly introduce the research project, the setting, the need for the project and the research question and objectives. I present an overview of mental health recovery and what this means for service delivery and then detail the ecological perspective that will underpin the overall structure and analysis of this thesis. I conclude this chapter with the structure for the overall thesis and what can be expected within each chapter.

Woodlands, the service under evaluation, approached Swansea University with regards to the possibility of a research project being conducted to evaluate the service. This meant that the idea for the research and part of the funding for this project came from Woodlands. In a reflection article I published during my PhD, I discuss the challenges of conducting research for an organisation when they are providing funding for the project (Pritchard, 2018). These challenges included individuals not understanding the research process, resistance towards the research and organisations having unrealistic expectations of what can be achieved within the timeframe.

\subsection{Woodlands}

I will use the pseudonym Woodlands throughout this thesis to protect the anonymity of the service, staff and service-users accessing the service. Whilst I note that the uniqueness of Woodlands may reveal the identity of the service, it is not possible to omit these factors 
because they provide contextual insights that are relevant to the findings and overall discussion of this research.

Over ten years ago, Members and Trustees of a mental health charity formulated a vision for an in-patient mental health service. Through campaigning and the financial support of Big Lottery and the Welsh Government, this was realised through the development of Woodlands Recovery Centre (Woodlands, 2019). Woodlands is the United Kingdom's (UK) first not-for-profit in-patient service delivered by a third-sector organisation. The service provides recovery-oriented care (ROC) for adults with serious mental illness, usually with a primary diagnosis of schizophrenia, who are subject to certain provisions of the Mental Health Act 1983. Woodlands has the following exclusion criteria:

- Persons under the age of 18 or over the age of 65 ;

- Persons with a primary diagnosis of personality disorder;

- Persons primarily presenting with alcohol or drug dependency;

- Persons with a primary diagnosis of Learning Disability;

- Persons with an Organic illness such as Alzheimer's Disease.

The design, implementation and delivery of Woodlands was influenced by numerous consultations held over a period of three years with service-users and carers across Wales. The overall aim of Woodlands was to enable service-users to lead an independent life, and where possible, support these individuals to move successfully back into the community. Woodlands aimed to achieve this through promoting choice, responsibility, self-determination, independence and recovery within a safe and supportive environment (Woodlands, 2019).

\subsection{The Context in which Woodlands Operates}

In this section I will consider the wider context in which Woodlands was operating. I will discuss the different conceptualisations of mental health recovery and how neoliberal ideology may be influencing these conceptualisations and how mental health recovery is being operationalised within practice. The current policy landscape for mental health and 
the demand for inpatient care in Wales will also be noted as this provides important context to some wider influences relevant to the new recovery-oriented service, Woodlands.

\subsubsection{Different Conceptualisations and Models of Recovery}

Recovery from serious mental illness is not a straightforward concept as it has been conceptualised in many ways. Recovery has been identified as both an internal and/or an external process, an outcome and/or a journey, and a clinical and/or a socio-political goal (Watson, 2012; Jacob et al., 2015). Recovery is, therefore, a nebulous construct (Watson, 2012); due to its complexity, there is not one accepted way of conceptualising it. However, different perspectives have been proposed and developed, within two main schools of thought, clinical and personal recovery (Davidson \& Roe, 2007), which I will now consider in some detail and the models of care associated with these conceptualisations.

\subsubsection{Clinical Recovery and the Medical Model of Care}

Clinical recovery refers to the amelioration of symptoms, whilst working towards what might be deemed normal functioning, or a cure. A clinical understanding of recovery emanates from a branch of medicine, psychiatry. Recovery is located within an illness framework of understanding and links recovery with a pervasive reduction or complete removal of symptomology (Slade et al., 2008). There are four key features of clinical recovery:

- "Recovery is an outcome or a state, generally dichotomous - a person is either 'in recovery' or 'not in recovery'

- It is observable - in clinical language, it is objective, not subjective

- It is rated by the expert clinician, not the patient

- The definition of recovery does not vary between individuals" (Slade \& Longden, 2015, pg. 3)

Clinical recovery comprises full symptom remission and although not a term used in the definition, this could be summarised as being 'normal' (Slade \& Longden, 2015). As the clinical model locates problems of mental ill-health largely within the individual, clinical 
endeavours focus on changing people through treatment so that they "fit in", i.e., become "normal" and "independent" of support and services (Slade et al., 2014).

The medical model assumes that mental illnesses are brain diseases, caused by abnormalities in the brain, therefore biological treatment is necessary (Andreasen, 1985). The pharmacological treatment of mental ill-health is based on the premise that medications can restore chemical imbalances in the brain, making recovery possible. This approach therefore focuses upon the problems and failures in people with mental illnesses.

Clinical recovery is associated with a model of care where there is a hierarchy and power imbalances, where those trained within medicine are seen to the experts and serviceusers are passive recipients (Cornuz et al., 2011). This has resulted in a paternalistic approach to decision-making where professional staff make decisions on behalf of the service-user and individuals are not involved in decisions about their care. Power imbalances are also reflected within Mental Health Act (2007) legislation in England and Wales as health professionals have the power to detain, assess and treat individuals with mental ill-health, in the interests of their own health or safety, or for the protection and safety of others. The assessment and management of risk is therefore a key component of the mental health system within the UK. The formalisation of risk has resulted in service users becoming defined in terms of the risk they present, rather than in terms of their needs and rights (Langan \& Lindow, 2004). Furthermore, it could be argued that mental health policies encourage a risk-averse approach, as professionals are concerned with the potential consequences of supporting positive risk-taking. This approach to risk serves to undermine the recovery approach as it has been criticised as encouraging coercion and containment (Boardman \& Roberts, 2014; Perkins \& Repper, 2016).

Traditionally, clinical recovery and the medical model of recovery has been adopted by mental health services and professionals within the field, with some suggesting it still dominates current practice (Slade et al., 2014). The outcomes of importance are clinical focused upon symptoms, health service utilisation, functioning and health outcomes. The challenge associated with clinical recovery and the medical model of recovery is that clinicians pathologise what is deemed symptoms of mental ill-health, and service-users and other aspects of their life are placed on the periphery (Slade \& Longden, 2015). 


\subsubsection{Personal Recovery and the Recovery Model of Care}

More contemporary understandings of recovery focus on a service user perspective which conceptualises that symptomatology can still be present, as recovery is about overcoming difficulties to the extent that the person feels they have regained control over their life. The recovery movement of the 1970s was an advocacy approach by 'survivors' of mental health services, who argued that they, and others like them, were entitled to a life beyond the stigmatising label of 'mental patient' (Davidson \& Roe, 2007). The recovery movement therefore afforded an alternative to the psychiatric, medical model that dominated the mental health sphere. Personal recovery is conceptualised as:

- "a process or a continuum

- is subjectively defined by the person themselves

- is 'rated' by the person experiencing the mental health difficulties, who is considered the expert on their recovery.

- Recovery means different things to different people, although there are aspects that many people share" (Slade \& Longden, 2015).

In contrast to clinical recovery, personal recovery is not about a cure but is instead focused upon recovering a life (Slade et al., 2014). The notion that individuals with mental ill-health need to be offered treatment so that they fixed or changed to fit in and become normal is not consistent with the values underpinning personal recovery. Personal recovery challenges how mental health practice are organised and delivered by ensuring individuals with a mental illness have opportunities and resources to lead a meaningful and purposeful live (Davidson et al., 2005), not just a focus upon symptoms and a cure.

The vision of this recovery was for individuals with severe and enduring mental illness to have greater independence and control as well as develop a life and identity beyond their mental illness (Slade, 2009). It recognised that there was more to an individual than their illness, and service-users can and should determine the direction of their recovery journey (Oades et al., 2005). Conceptualising recovery in this way means that it is highly individualised and therefore difficult to establish a definitive view of personal recovery (Leamy et al., 2011); meaning embedding this concept into mental health practice has become a complex task, and could be a contributing factor as to why some argue this 
understanding of recovery remains rhetoric rather than routine (Slade et al., 2014). Despite the challenges of operationalising personal recovery, this model of care attempts to:

- Establish equal partnership working which are compassionate, respectful, hopeful and future-focused (Repper \& Perkins, 2012)

- Shift away from a dominance of pharmaceutical treatments and diagnostic labelling to valuing the individual as a person with wishes, hopes, fears and beliefs and who is an expert in their own life (Thornton, Crepaz-Keay, Birch, \& Verhaegh, 2017)

- Focus on strengths rather than deficits (Davidson et al., 2009)

- Professionals adopt a supporter role rather than a role of 'fixer' or 'expert' (Repper \& Perkins, 2012)

- Provide a broad, holistic range of community-oriented services (including, but not limited to, housing, education, employment, support in everyday living, drug treatments and talking therapies), and promoting social inclusion and human rights (Slade et al., 2014)

- Support individuals to live a meaningful and purposeful life, by promoting care predicated on human rights and a social model of exclusion. Inclusion and citizenship are not about becoming 'normal' or 'independent', but about creating inclusive communities that accommodate everyone and ensuring that support is available for individuals to have a full and equal participation for all in society (Slade et al., 2014).

Deegan (1988) suggested that traditional Western values, such as self-sufficiency, individualism and personal achievement are projected onto individuals with a mental illness. She argued that for some, especially those who relapse frequently, these traditional values are oppressive and are invitations for failure.

"For these persons, "independent living" amounts to the loneliness of four walls in the corner of some rooming house. For these persons, "individual vocational achievement" amounts to failing one vocational program after another until they come to believe they are worthless human beings with nothing to contribute. For these persons, an alternative type of rehabilitation program, and even lifestyle, should be available as an option. 
Instead of competitive vocational training based on individual achievement, a cooperative work setting stressing group achievement could be established." (Deegan, 1988)

This highlights for individuals with severe and enduring mental illness, an alternative way of living and treatment, that is not grounded in Western ideals, is necessary to support recovery. Anthony (1993) built upon Deegan's (1988) work, which was based upon her lived experience, captured the individualised nature of recovery:

"Recovery is a deeply personal, unique process of changing one's attitudes, values, feelings, goals, skills and/or roles. It is a way of living a satisfying, hopeful, and contributing life even within the limitations caused by illness. Recovery involves the development of new meaning and purpose in one's life as one grows beyond the catastrophic effects of mental illness." (Anthony, 1993)

Anthony's (1993) conceptualisation of recovery was considered in relation to individuals with severe and enduring mental illness, such as those labelled schizophrenia and bipolar conditions, however this concept has since been extended to include diagnoses such as first-episode psychosis and depression (Stickley \& Wright, 2011). Anthony's (1993) definition of recovery acknowledges the lived experience of each individual and emphasises the personal nature of the recovery journey. However, one criticism of this definition is that it does not capture the influence of social and political factors, nor does it emphasise the stigma, exclusion and discrimination that hinders individual's recovery journey (Pilgrim, 2009). Anthony's (1993) definition could further be criticised for locating the responsibility for recovery with the individual, and if this is not achieved, this may leave room for implicit blame. Additionally, a narrow focus upon the individual serviceuser, may have the potential to limit recovery, and overlooks our knowledge that social processes can influence recovery (Ungar, 2013; Recovery in the Bin, 2019). According to the personal recovery approach, it is society that needs to change, not people (Slade et al., 2014). This view of recovery aligns with a social model of disability (Oliver, 2004), which service-user activist group "Recovery in the Bin" (2019) identify as the model that should be underpinning recovery for those with severe and enduring mental illness.

The social model of disability is concerned with the impact the wider context has upon a person's health and participation in society (Helman, 2007). Pilgrim (2009) described how 
contextual factors such as government policy, poverty, poor housing, and discrimination are influential in health and recovery. The model advocates that the root causes and consequences of an illness are important and need to be addressed (Blaxter, 2010). This is important because some research suggests that it can be more difficult to recover from the consequences of the mental illness, such as loss of social roles, poverty and poor housing (Borg et al., 2005; Davidson et al., 2005), than the symptoms associated with the illness (Anthony, 1993). This highlights the importance of wider contextual factors upon recovery, and how these could shape individual's recovery journey, and their ability to live a life beyond the stigmatising label of 'mental patient' (Davidson \& Roe, 2007), which is central to the recovery movement (Deegan, 1988; Anthony, 1993).

Despite the appeal of a conceptualisation of recovery that originates from service-users, similarly to clinical recovery, it is not without its difficulties. One criticism is that it is difficult to identify a shared definition because it may mean different things to different people (Slade \& Wallace, 2017), meaning the concept of personal recovery is vague (Beresford, 2015). This vagueness therefore makes researching the concept difficult and complicates its practical application within services (Slade, 2009).

\subsection{Recovery and Rehabilitation}

Rehabilitation and recovery are often used interchangeably, however there have been calls for these terms to be differentiated from one another (Slade et al., 2014). Serviceusers rarely refer to rehabilitation, but instead talk about a non-linear, personal process of getting well and getting on with their lives (Andresen et al., 2003). Rehabilitation, on the other hand, involves targeted interventions to assist individuals to acquire, relearn and apply skills as well as access support and resources required to live a meaningful life in the community (Mitchell, 2003). It can therefore be said that recovery is the subjective experience, beliefs, wishes and desires of the individual, whereas rehabilitation is the services provided to the individual. According to Anthony (1993) services cannot create recovery for an individual but can provide means that can facilitate recovery. This raises the question as to whether services, even if designed and developed with service-user and carer input, can ever be recovery-oriented, as this is relates to an individual experience. It also highlights that there are already services aimed at supporting those 
with complex mental ill-health to support them to gain independence and a life outside of services, which have also attempted to incorporate recovery principles into practice, therefore it raises the question of how Woodlands considers itself to be providing something unique and different to these services.

\subsubsection{Recovery as Policy}

Policy may be an important context, and even a catalyst, for trends towards recoveryvalues, but should be considered an attribute of the paradigm, rather than constitutive of it. Whilst this study is not about the impact of mental health policies upon Woodlands, the policies are an important context and background for this research focus. Due to this, I will provide details of the relevant mental health policies operating within Wales and will be influencing the operations at Woodlands.

Many countries, including the United Kingdom, have adopted the concept of personal recovery into policy to organise treatment and care of mental ill-health in a recoveryoriented way (Ramon et al., 2007). As the concept of recovery has become a prominent feature of both mental health research and policy, there has been an increasing expectation for major reform within the mental health system to deliver recoveryoriented care (ROC). It is now widely considered that mental health services should be recovery-oriented and guided by personal recovery. This is despite literature which reports that a medicalised view of recovery still prevails within mental health services (Morera, Pratt, \& Bucci, 2017) and recovery-oriented services remain sporadic (Le Boutillier et al., 2015).

In the UK, recovery and ROC is supported within various government policies that promote self-management and choice for long-term conditions (Department of Health, 2001; 2006; 2008a; 2008b; 2011). In 2007, the Department of Health published a 'commissioning framework for health and well-being' which emphasised the importance of mental health services providing support to help people integrate into their communities (Department of Health, 2007). These policies identified many factors that could support individuals in their recovery from mental ill-health and help them to have a good quality of life. These factors included, but where not limited to, better employment rates, improved changes in education, a greater sense of purpose and stronger social 
relationships (Department of Health, 2011). A government programme was established in 2008 to guide services in being supportive of recovery for service-users (Shepherd et al., 2014). The initiative focused on enhancing hope and optimism, promoting a sense of agency, and introducing shared decision-making, person-centred care and selfmanagement into practice.

The incorporation of recovery ideology into policy and services required a shift away from paternal models of care to a more collaborative approach where service-users were involved in decisions about their care and how they lived their lives. Instead of focusing on stabilisation, symptom relief and clinical goals largely dictated by professionals, ROC is based upon equal partnerships, person-orientation, person involvement, the promotion of hope and self-determination (Slade et al., 2014). The overall aim of ROC is to support individuals in gaining meaning and purpose in their lives through social inclusion, supportive relationships and attaining personal goals (Farkas, 2007). Forms of support such as developing self-help and coping skills to promote recovery and peer support. However, embedding recovery into policies in this way has become increasing contested over recent years (Recovery in the Bin, 2019).

\subsubsection{Challenges to Recovery as Policy}

Self-management and independence from services have become central to recent mental health policy within the UK (Department of Health, 2007;2011). This reflects the dominant approach within the field of mental health which focused primarily upon encouraging individuals to better integrate themselves into society. It has been argued that government attempts to incorporate recovery into policies has resulted in them taking ownership of the ideas and insights brought about by service-user activists (McWade, 2016).

In these policies individualistic narratives where distress is framed as illness remain, which serves to continue to empower psychiatry rather than service-users (McWade, 2016). Instead models that are humanistic, holistic and do not reduce individuals to a set of symptoms are needed; and individuals need to be understood within the social and economic context of the society in which they live (Menzies et al., 2013). Whilst the inclusion of recovery has been advantageous for some individuals as they are not 
dependent upon services; for others it has resulted in premature discharge and subsequent relapse (Slade et al., 2014). This shows that recovery cannot be a one size fits all approach, despite policymakers and services attempts to respond in this way. This highlights that attempts to operationalise personal recovery and incorporate it within policy to guide service delivery may in and of themselves be clear deviations of the true meaning of personal recovery which strives to support the individual experience of the service-user. This raises the question as to whether any service can truly provide ROC, as the values of the recovery movement cannot be neatly broken down into a generalisable set of principles, nor do those involved in the service-user movement want this (Deegan, 1988). This poses a challenging, potentially impossible, task for policymakers and service providers who are trying to incorporate recovery and ROC within policy and practice.

These policies have been developed when mental health services were facing significant issues with underfunding and austerity; leading to welfare cuts, undermining the security of those experiencing mental health difficulties and potentially increasing stigma, anxiety and exclusion (O'Hara, 2014). The context of austerity and recovery is important as it raises the question as to whether recovery is possible within the current socio-economic climate and arguably suggests that neoliberal ideology may have significantly influenced the incorporation of recovery in policy and continues to govern the wider mental health system influencing the way in which recovery is fulfilled within services.

\subsection{Recovery and Neoliberal Ideology}

The emergent issue of divergent views of mental health recovery, its inclusion within policy and its operationalisation might be explained by wider ideologies that operate in the background of service delivery. The mental health system continues to evolve and change as a result of various social, political and economic influences (Frank \& Glied, 2006). Due to this, mental health and service provision must always be understood within the current social climate as mental health policies are highly influenced by the values and concerns of the society in which they originate (Scheid \& Horwitz, 1999).

According to some, current policy and service provision have become deeply embedded within the ideals of neoliberalism (Morrow, 2013; Rose, 2014). This is an unsurprising critique as neoliberalism has been the overarching political driver since the 1970s (Wynne, 
2004) and remains the dominant ideology influencing policy development. Policy implementations of recovery emphasise a reduction in service dependency and provision (Ramanuj et al., 2015). Some view this as a veiled attempt to ration and dilute services (McKeown et al., 2017), and renders the concept of recovery as easily co-opted by a neoliberalist agenda (Recovery in the Bin, 2019). The Recovery in the Bin group (2019) campaign that this neoliberalist co-option is a cover for coercion, victim blaming and removal of services under a drive towards austerity, which they refer to as 'neorecovery'. It is therefore important I consider neoliberalism as an important backdrop to the context in which Woodlands was operating, and how this may implicitly, or explicitly influence Woodlands ability to fulfil its ambition of providing ROC. Although I will not specifically be researching neoliberalism within this project, it does provide an insight into the wider context in which Woodlands operates.

Neoliberalism is an ideology that emphasises an individual's responsibility, selfmanagement and their economic independence regardless of their social circumstances (Morrow, 2013). As a school of thought is characterised by privatisation, community run agencies and an emphasis upon the individual and the family to take responsibility for the vulnerable (Henderson, 2005). The imperative of neoliberalism is for individuals to selfregulate; which tasks those with a mental illness as responsible for constant selfimprovement and self-management of their health and wellbeing, removing the role of the state in supporting those in need. Under the welfare state it was argued that individuals were becoming dependent upon the system and therefore fostering selfsufficiency naturally reduces the dependency upon the welfare state and the associated financial burden (Brijnath \& Antoniades, 2016). Promoting mental health recovery using individualistic approaches, such as self-management as advocated in recent policies (Department of Health, 2007; 2011), present ways to reduce costs by providing rationale for the cutting of welfare benefits, services, and skilled staff, neatly fitting with austerity measures (Rose, 2014).

Mental health conditions are often treated as self-contained ailments that can be resolved individually, typically through pharmaceutical means, as opposed to being considered byproducts of a neoliberal society that promotes personal gain, competition and social alienation (Morrow, 2013; McWade, 2016). The promotion of these neoliberal ideals is a 
distinct deviation from service-user views of recovery which advocate for alternatives to self-management, individualism and competition (Deegan, 1988). This suggests that the progressive and alternative narrative that was borne out of the recovery movement has been adapted to promote neoliberal agenda of the UK government, under the guise of ROC. Some have suggested that the colonisation of recovery has been used to cover up service reduction, reduce welfare support and inappropriately shifts the burden of responsibility for recovery onto service-users (Recovery in the Bin, 2019; Aston \& Coffey, 2012). It raises the question if recovery is a personal experience what role is there for the state; further reinforcing a neoliberal agenda.

Mental health problems and the treatments used to support individuals in their recovery are largely divorced from social, economic and political contingencies and instead are positioned as personal pathologies that can be diagnosed and treated. Locating mental illness as an individual problem, that requires an individual solution negates the contribution of macro-level factors, such as poverty, work inequities and class (Peacock et al., 2014; Teghtsoonian, 2009). The shift towards individualism, albeit often presented under the guise of collaboration, co-productive and partnership, enables responsibility to be shifted away from the government, and onto the individual, or their family (Sullivan, 1994). Individuals with a mental illness are therefore responsible for modifying their behaviours, attitudes and addressing their problems to fit a normative pattern which promotes productive behaviour, happiness and a fulfilling life. If people fail to accept this personal responsibility this serves to reinforce the pathology of the individual, and position them as irrational, unproductive and deviant (Giroux, 2008). These neoliberal values shape what is regarded as responsible, productive and rational forms of human agency and pathologises thoughts and behaviours that deviate from these ideals (Soss et al., 2009). Government policies ally productive and economic contribution with mental health recovery through prioritising service outcomes such as securing employment over the wellbeing of the individual. This moralistic position leads to understandings of mental ill-health as a failure of personal accountability, rather than a failure of the state (Ayo, 2012), which serves to alienate individuals with mental ill-health, rather than support them to establish a meaning and purpose within society. 
The marketisation of a mental health system that promotes the ideals of selfmanagement, individual autonomy and self-sufficiency works against the collectivist and social justice enactments of recovery that underpin service-user narratives of recovery (Deegan, 1988; Recovery in the Bin, 2019). Instead of the social change sought by recovery and service-user activists, it has been suggested that existing practices have been coopted and rebranded as recovery-oriented (Rose, 2014; Recovery in the Bin, 2019). Mental distress is not understood in terms of a response to social injustice and relative deprivation, but instead as individual pathology (Friedli, 2009), which continues to marginalise, medicalise and exclude individuals with mental ill-health. The overall message of neoliberalism is that there is no place in society for unsuccessful people and that a zero-tolerance approach to welfare dependency is necessary (Johnson, 1990). Suggesting that there is a division between who is a deserving and undeserving person, largely based upon their earning power (Beresford, 2005). These ideological underpinnings work against the recovery movement and shows the conflict surrounding what recovery and ROC are. It can therefore be asserted that recovery remains a site of contested meaning, which muddies the landscape in which Woodlands operates.

It could be argued that the enactment of recovery-as-policy, in Wales and the UK, is a form of neoliberal state-making that is designed to fail some more than others, most notably individuals living in poverty, or from ethnic minorities (Tyler, 2010). This may be contributing to the reported discriminatory and unjust mental health system that some service-user advocates are experiencing (Recovery in the Bin, 2019).

The influence of neoliberal ideology upon the conceptualisation and operationalisation of ROC within policy appears to be fuelling the divergent views on recovery, and ongoing conflict of whether recovery should be operationalised, and how this should be achieved. Undoubtedly, the development, design and delivery of Woodlands will have been influenced by the current dominant ideology, neoliberalism. Even if the service's intentions were to deliver a service informed by service-user narratives, the way in which policies have conflated self-management, self-sufficiency and independency with recovery and $\mathrm{ROC}$, seems to be a deviation from the ideals sought after within the recovery movement. Furthermore, even if mental health services are encouraged to consider personal and social outcomes, this will always come with limits; for example, it 
is unlikely that service-users who aspire to go to bed for a month will be supported or accepted by even the most recovery-focused services (Rose, 2014). This shows that there is no easy, or simple, way to operationalise personal recovery, and shows a real challenge that Woodlands is likely to face.

\subsubsection{The Ongoing Challenge}

Recovery as a conceptual frame, and as a set of policy orientations, encapsulates on-going debates concerning mental ill-health and its management. Whilst I have shown the multiple threads of recovery, its conceptualisation is still in process; and therefore, cannot be understood to belong to any particular moment or movement in the mental health sphere. These varying conceptualisations of recovery highlight that at present there is a lack of homogeneity in what we mean by recovery. This has created a landscape that is forever shifting, meaning our understanding of recovery, and subsequent attempts to capture it within policy, and operationalise it within practice, have become complex and muddy. The fact that there a several different models of care still operating within the mental health sphere is unexpected seeing as there a lack of consensus of what recovery is persists. In the current landscape clinical recovery remains ingrained within services, this, combined with the vagueness of personal recovery, means that there are inevitable misunderstandings about what recovery means for services users (Tickle et al., 2014). Despite these complexities, policymakers have still introduced recovery as a central tenant of current policy, and it remains an expectation of service providers.

\subsubsection{Mental Health Policies in Wales}

Mental health services in the UK have seen, a policy shift from institutional care characterised by paternalism, harm and disenfranchisement, to community-based services and partnership working. This has been reflected within policy which promotes least restrictive practice, prudent healthcare and early intervention within primary care to prevent admission to inpatient services.

In Wales, the Government has chosen to use legislation to drive improvements in mental health delivery, recovery and care continuity (Glasby \& Tew, 2015). The Mental Health (Wales) Measure 2010 has focused on augmenting primary mental health services whilst 
enhancing care coordination and planning with specialist, secondary services through using the mandatory Care and Treatment Plan (Welsh Government, 2011, 2015). The Measures has some guiding principles at the core of its design and are expectations all services will be held to. These principles are: service-users and carers being involved in the planning, development and delivery of care and treatment, clear communication to ensure service-users and their carers are involved in their care, care is holistic and personfocused, care should be integrated and coordinated, care should be proportionate to need and risk, and lastly care is characterised by equality, dignity and diversity. These underlying principles appear to be in keeping with the personal recovery narrative. These principles position service-users as active and equal participants within their care and treatment, rather than passive and powerless recipients; and care is focused upon all aspects of the individual, not just their mental illness which supports the notion of serviceusers being treated as a person, not just a set of symptoms. The operationalisation of how these principles are going to be achieved is divided into four main parts which I will now discuss.

- Part 1 - improve local primary mental health services through introducing enhanced practices, such as comprehensive mental health assessments, short term psychological treatment and greater provision of information about treatment and signposting to other services (Welsh Government, 2011). There is an emphasis placed upon self-management and independence through increasing treatment in the community, as well as reducing dependency upon secondary services.

- Part 2 - a mandatory requirement for all those accessing secondary services and have a serious mental illness must have a Care and Treatment Plan and be assigned a care coordinator (Welsh Government, 2011). Several individuals with a serious mental illness were discharged to primary care (Gofal, 2015), whilst a more select group of people with serious mental illness remained at the secondary level, for whom this level of care was deemed appropriate. This can be considered to correspond with prudent healthcare principles that also govern Wales, as there is a focusing of resources on individuals with the greatest need (Aylward et al., 2013). 
This suggests that those accessing specialist, secondary services have the greatest level of need and therefore may have more complex and severe mental ill-health.

- Part $\mathbf{3}$ (which is not of particular relevance for this study) - provide a means for individuals to refer themselves back to secondary services within three years of discharge (Welsh Government, 2011).

- Part $\mathbf{4}$ (which is not of particular relevance for this study) - provide a statutory scheme of mental health advocacy (Welsh Government, 2011).

This legislation represents a shift away from traditional service models for individuals with serious mental illness, which has been delivered by secondary care services (Gilburt et al., 2014). Previously primary care operated as a gatekeeper to specialist secondary services, whereas now it is argued that primary care can take a lead role in mental health service delivery (World Health Organisation, 2008; Currid et al., 2012). Whilst the emphasis upon the role of care reflected within policy and legislation has been associated with a recovery approach, it also has engendered financial savings as specialist service provision have reduced and primary care offers a cheaper alternative to hospitalisation (Ramanuj et al., 2015). This raises the question as to whether the shift to primary care, under the auspices of recovery, will improve services, service-user's quality of life and their recovery, or whether the latest cost-saving transformation may paradoxically result in poor quality care and treatment that deviates from adopting the personal recovery narrative, and instead promotes 'neorecovery' (Recovery in the Bin, 2019).

The Mental Health (Wales) Measure 2010 emphasis upon self-sufficiency, selfmanagement, and independence through increased treatment within primary care, could therefore be situated within the neoliberal consensus of recovery, and can be seen as an instance where personal recovery has been colonised and its true value distorted.

\subsubsection{Service Provision and Current Demand for Inpatient Care}

The current study is centred upon an inpatient service within Wales. The provision of inpatient care has evolved greatly in the UK and has moved through phases of mass institutionalisation to the current drive which is to provide more care in the community. 
In the 19th century, the UK constructed large-scale asylums designed for the care of the mentally and physically unwell. By the 1900s large numbers of people who were considered unable to function in the community for physical or mental health reasons were held and treated in the asylums (Fakhoury \& Priebe, 2007). However, poor conditions and levels of care in these institutions led to their close in the 1950s (Fakhoury \& Priebe, 2007), and in the following decades smaller discrete units were established. The reduction in bed numbers pathed the way for an increased focus upon treating individuals in their own home through community treatment, driven by the 'Caring for People: Community care in the next decade and beyond' government document. This has resulted in numerous changes to the inpatient landscape for mental health.

In 2018-19, there were 8315 admissions to mental health facilities in Wales. 97\% ( $n=8098)$ of admissions in 2018-19 were to NHS facilities in Wales, with the remainder admitted to independent hospitals ( $n=217)$. The number of admissions in Wales has steadily fallen in the past ten years between 2009-2010 and 2018-2019 to stand at 8315, a decrease of 3041 (27\%). This decrease was largely driven by informal admissions which fell from 9904 in 2009-2010 to 6339 in 2018-2019, a decrease of 35\%. In contrast, formal admissions rose from 1452 in 2009-10 to 1916 in 2018-19, an increase of 32\%. It appears that inpatient care is being reserved for those persons who are sectioned under the Mental Health Act, suggesting inpatient services are supporting those most at need, aligning with the prudent principles in Wales and a neoliberal agenda on healthcare. The overall decrease in the use of inpatient settings is largely attributed to individuals accessing support in the community, or in primary care. The decrease in the number of individuals accessing inpatient care is coupled with a decrease in the number of NHS beds available over the past 20 years (see Table 1 ).

\begin{tabular}{|l|c|c|c|}
\hline \multirow{2}{*}{ Year } & \multicolumn{3}{|c|}{ Mental Health NHS Bed Availability } \\
\cline { 2 - 4 } & $\begin{array}{c}\text { Average daily } \\
\text { available beds }\end{array}$ & $\begin{array}{c}\text { Average Daily } \\
\text { Occupied Beds }\end{array}$ & $\begin{array}{c}\text { Percentage } \\
\text { Occupancy }\end{array}$ \\
\hline $1998-1999$ & 1169.9 & 1051.0 & 89.8 \\
\hline $2008-2009$ & 880.9 & 756.5 & 85.9 \\
\hline $2018-2019$ & 722.4 & 672.6 & 93.1 \\
\hline
\end{tabular}

Table 1 - NHS Bed Availability 


\subsubsection{Independent Services}

21 independent hospitals are registered with Healthcare Inspectorate Wales as of October 2020, of which Woodlands is one. I will now show the current service provision of independent services in relation to categorisation of service, whether it provides services to males or females, and who provides the service. One service was excluded as this provided services to children/young people, as opposed to adult mental health. I have put an asterisk in the table next to the categorisation that is relevant to Woodlands (see Table 2).

\begin{tabular}{|c|c|}
\hline Independent Hospital & Total \\
\hline \multicolumn{2}{|l|}{ Categorisation } \\
\hline Medium Secure & 0 \\
\hline Medium and Low Secure & 1 \\
\hline Low Secure (including forensic services) & 4 \\
\hline Low Secure and Rehabilitation & 1 \\
\hline Locked Rehabilitation* & 11 \\
\hline Open Rehabilitation & 1 \\
\hline Open, Rehabilitation and Low Secure & 1 \\
\hline Specialist Service for Organic Brain Disorder, Dementia or Acquired Brain Injury & 1 \\
\hline \multicolumn{2}{|l|}{ Sex of service-users } \\
\hline Male & 7 \\
\hline Female & 3 \\
\hline Both (either in the same service, or in separate services) * & 10 \\
\hline \multicolumn{2}{|l|}{ Provider } \\
\hline Elysium & 6 \\
\hline Priory & 4 \\
\hline Ludlow Street Healthcare & 3 \\
\hline Mental Health Care (UK) Ltd & 2 \\
\hline Cygnet & 1 \\
\hline Coed Du Hall Ltd & 1 \\
\hline Regis Healthcare & 1 \\
\hline Rushcliffe Care Group & 1 \\
\hline Third-sector charity * & 1 \\
\hline
\end{tabular}

Table 2 - Independent Mental Health Hospitals in Wales 
These tables show that independent hospitals typically are classified as providing rehabilitation services to individuals with a mental illness and it typically caters for both sexes either at the same service, or on separate male and female wards. Woodlands is, however, the only service to be provided by a third-sector charity organisation. The NHS Wales Quality Assurance Improvement Service promotes that NHS-run services are prioritised above outsourcing to independent hospitals.

Inpatient facilities are therefore reserved for those at greatest risk and who have the greatest needs. A freedom of information request to the local health boards in Wales revealed that individuals with a personality disorder, self-harm and autism were the current demographic being referred to locked rehabilitation services, however due to the limited number of service-users in locked rehabilitation for the protection of these individuals the local health boards were only able to provide minimal information.

\subsubsection{Why is the Current Political and Policy Landscape Important to this Project?}

Understanding the sphere in which Woodlands is operating is of great importance. Whilst it is not the focus of this project, it provides context to the expectations, challenges and complexity that exists within mental health service delivery and recovery. I have shown that there is a lack of clarity surrounding the concept of recovery which adds to the difficulty of services fulfilling the policy rhetoric of ROC. The limited consensus of what recovery means, or how it can be achieved in practice, makes Woodlands ambition and drive to deliver a service underpinned by a concept that remains contested interesting. I have also shown that the current political drive of austerity and neoliberalism has resulted in recovery being conflated with self-management, self-sufficiency and independence from services. As this is the current dominant ideology guiding policymaking in the UK, it will be interesting to see if Woodlands has implicitly or explicitly been influenced by this interpretation of recovery. I have noted that there has been a shift towards communitybased care rather than inpatient care, therefore it will be of interest to see whether Woodlands, which is a locked service, is able to establish itself as a provider and that there is a current need for this type of service within South Wales. 
If Woodlands can overcome these wider socio-political challenges then the findings of this project could be meaningful for other inpatient services who should also be providing ROC.

The current policy landscape and inpatient provision shows that there has been a focus towards achieving recovery focused services, which are guided by the personal recovery narrative borne out of the service-user movement. Therefore, an evaluation of a service like Woodlands is of relevance as the service is attempting to fulfil the policy rhetoric of ROC, and will help to develop our knowledge of how services are trying to do this and what is, or is not, working in inpatient settings when trying to achieve this policy expectation. There is a trend which shows there has been a decline in the number of NHS inpatient beds available; coupling this with the knowledge that there is a policy drive to treat individuals in the community (where appropriate) it will be interesting to see whether there is a need for an inpatient service like Woodlands. Especially when NHS mental health inpatient beds are not being used at full capacity, community-based services are being increasingly used and a number of other independent services are classified as providing the same categorisation of care as Woodlands.

\subsubsection{How Does Woodlands Claim to be Different?}

According to Woodlands' website and brochures they have identified several areas of service delivery that they believe make the service 'unique' or 'pioneering' (Woodlands, 2019), which I will now highlight:

- This is the first inpatient setting to be delivered by a third-sector organisation, as opposed to services which are delivered by the NHS or large private providers;

- Service-users and carers were consulted during the development and design of Woodlands;

- Woodlands claims that other services are not fulfilling policy expectations of ROC and that is what makes them different as they have a clear intention to put recovery values and what service-users want into practice;

- Service-users make decisions about the day-to-day running of the service (e.g. what activities there will be and what they will eat and make for dinner), rather 
than using a predefined structure of activities, or meals being made for them at set times;

- A team of peer mentors are employed, and they form a key component of the multidisciplinary team;

- The approach to risk and choice is not risk-adverse, and supports service-users in all their choices to provide opportunities for individuals to learn from their choices;

- The organisation operating Woodlands provides 'step-up' accommodation and numerous community-based services so that service-users can move on stage-bystage towards independent living. 'Step up' accommodation refers to a house which is opposite to Woodlands where service-users would be responsible for the running of the house (e.g. paying bills, cooking, cleaning, budgeting);

- "We do not maintain our Guests in their condition; instead we work with them to make progress towards goals in all areas of life";

- Woodlands is service-users home, not a clinical, hospital environment;

- The service is underpinned by a "unique Recovery Programme", which promotes self-management and a holistic approach to recovery.

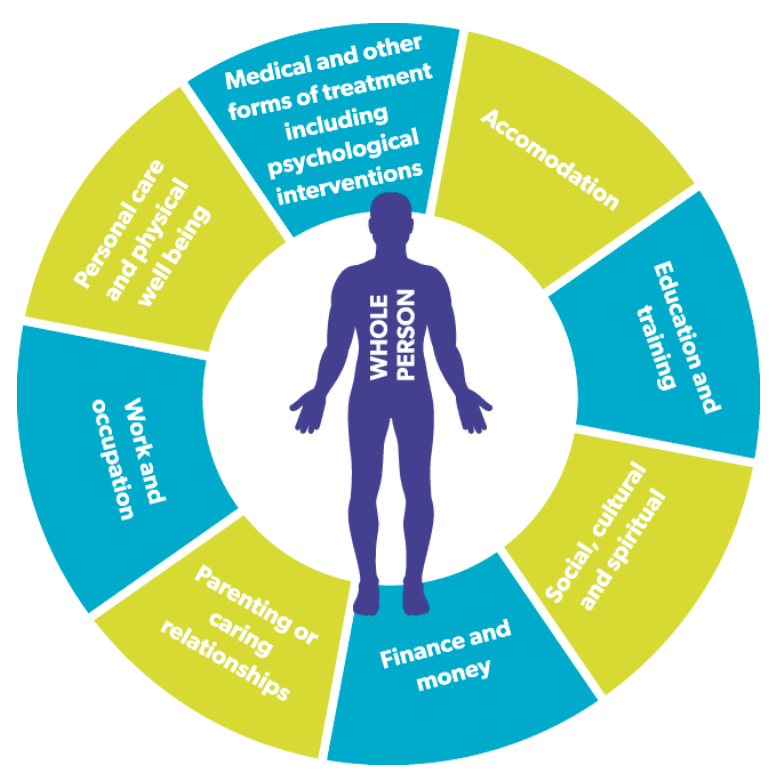

Figure 1 - Recovery Programme used at Woodlands

Woodlands suggested that although there are policies and expectations in place that all services will operate in this way, this is not being achieved, whereas it is Woodlands 
intention from the outset to design and deliver the service in a recovery-oriented way. However, it is important to note that although the organisation who operate Woodlands are credited for the Recovery Programme, the uniqueness of it within mental health practice can be contested as it is now used as the mandatory Care and Treatment Plan within the Mental Health (Wales) Measure 2010 (Welsh Government, 2011). This suggests that all services within Wales will be expected to providing care which covers the above areas and may not position Woodlands as unique as they claim.

\subsubsection{The Need for the Project}

Research into ROC in inpatient contexts remains in its infancy. The research available has focused primarily on ROC in community and outpatient settings (Salyers et al., 2007), with little attention given to its implementation and effect in inpatient contexts (Davidson et al., 2016). It is, therefore, imperative that the policy shift towards ROC is researched in inpatient settings to understand whether, and how, these settings are achieving ROC and whether this is having an influence on service-users' recovery. Developing our knowledge of how, why and for whom ROC in inpatient settings works, could begin to build an understanding of what contexts are conducive with the successful working and delivery of ROC and lay the foundations for much needed future research. In Chapter 2, I discuss the available research relating to ROC in inpatient settings.

\subsection{The Research Question and Objectives}

The aim of this research was to evaluate a recovery-oriented inpatient service and to understand how and in what circumstances the service supported individuals in their mental health recovery. The research question of a realist evaluation is "What works, for whom and in what circumstances at Woodlands in supporting service-user recovery?". To address this and increase knowledge surrounding how ROC works in inpatient settings, I will address several more specific research objectives:

1. To identify the key resources (mechanisms) used at Woodlands and to identify how these resources are working to support service-users in their recovery? (see Chapter 5 and 6). 
2. To identify the contexts that are, or are not, conducive for these resources to work as intended in supporting individuals in their recovery (see Chapter 4, 5 and 6).

3. To develop programme theories which lead towards un/successful delivery of recovery-oriented care at Woodlands (see Chapter 4,5 and 6).

4. To develop transferable theory of wider value to the mental health sector (see Chapter 8).

An additional research objective was included in the project once I had conducted several interviews in which participants claimed Woodlands had trouble in securing referrals. As Woodlands was a new service trying to establish itself within the wider mental health system, I decided to consider Woodlands as a resource that was being embedded in a preexisting context, the mental health referrals system. Therefore, I considered it necessary to collect and analyse data specifically relating to Woodlands as a resource being introduced into the competitive and commercial marketplace for mental health service provision. The following research objective was therefore added:

5. To identify why Woodlands faced challenges when trying to establish itself as a new service within the mental health referrals system? (See Chapter 4).

\subsection{Thesis Structure}

This thesis is divided into eight chapters, I will now present an overview of the 7 chapters that remain.

In Chapter 2, I outline the method and design utilised to answer the research question and address the research objectives. This includes an introduction to realist evaluation (RE), including the process, key terminology, and why RE was considered appropriate for this thesis. I also provide an overview of the process surrounding data collection, data analysis and the ethical considerations relevant to the project. The decision was made to 
put the methodology chapter before the literature review, because the literature review was informed by the underpinnings of realist evaluation and therefore, I wanted to ensure these concepts were presented before using them to guide my work.

In Chapter 3, I present a review of the available literature on ROC in inpatient services, in which I consider how services are attempting to deliver ROC, current barriers, as well as contextual factors that are, or are not, conducive to the delivery of ROC in inpatient settings.

In Chapter 4, I present my analysis of participant accounts relating to how Woodlands operated within the wider context of the mental health system. This chapter relates specifically to the exosystem of the EST (see Figure 3).

In Chapter 5, I present my analysis of participants' accounts relating to the staffing model that was intended to be delivered at Woodlands. This chapter relates specifically to the interrelations between the micro-level and the service-user, as well as the influence of the exosystem on service delivery at the micro-level (see Figure 3).

In Chapter 6, I present my analysis of participants' accounts concerning how service-user choice and responsibility was promoted at Woodlands. This chapter relates specifically to the interrelations between the individual and the micro-level, as well as the interactions between the macro-level and the micro-level (see Figure 3).

In Chapter 7, I present my analysis of the routinely collected outcome data that was made available to me from Woodlands. I present six clinical case studies to highlight patterns in the data that display a range of outcomes.

Finally, in Chapter 8, I discuss my findings and attempt an integration of the different data sets, and consider what this means for Woodlands, practice and the current evidence base. I position these findings in relation to the research objectives (see section 1.4), as well as considering limitations and directions for future research. 


\subsection{Locating the Researcher}

The researcher should always locate themselves within the study (Etherington, 2004). Study findings will be affected by the values, perspectives and cultural biases brought by the researcher to the inquiry (Guba \& Lincoln, 1994). The researcher is, therefore, an active agent in producing meaning and knowledge about the world in the process of coconstructing it with participants (Denzin, 1994). The researcher should therefore identify themselves as an important facet of the research inquiry, and practice reflexivity. Knowledge is a reflection of the researcher's social, political and cultural context and this can influence the focus and direction of the study. Reflexivity is understood as an ongoing process whereby researchers reflect upon their role in contributing to data collection and analysis through critical self-examination (May \& Perry, 2013). However, the notion of reflexivity is contested as being ambiguous and subjective (Lynch, 2000). I will now consider some personal and professional experiences that I reflected upon prior to data collection and analysis.

I am a white, British, middle class woman. I undertook this PhD because of an interest in mental health recovery, and my desire to understand the role of services in supporting individuals in their recovery journey. Close family members of mine have had difficulties with their mental health and I took an active role in supporting them through these challenges both in my child and adult life. This had meant I have encountered first-hand the difficulties people can experience when trying to overcoming these challenges.

Due to this I have always wanted to be in a position where I can support others in their recovery. This led me to complete an BSc in Psychology and a MSc in Forensic Psychology and eventually employment within a Psychology department in a secure, forensic mental health service where individuals were sectioned under the MHA. In my clinical role as an assistant psychologist, I encountered resistance from colleagues when working therapeutically with service-users and would be asked why I would want to help him/her based on their offences, or when acutely unwell. From these experiences, I became more interested in what was meant by the term recovery and how this was supposed to be supported by staff and services. I became interested in what role I could play in supporting others with their mental ill-health. I reflected that my personal experiences of family 
mental ill-health and the therapeutic pessimism I encountered in inpatient services may influence my approach to data collection and analysis when evaluating a service attempting to support individuals in their recovery.

During my role as an assistant psychologist I became aware of the Hearing Voices Group and advocated for the service to support service-users to establish this group. It was at this point I was exposed to the role of lived experience, and service-user defined recovery, which resonated with my experiences of mental ill-health in my own family. I was aware that I often aligned with the service-user narrative of recovery, given my experiencing of mental ill-health within my own family, so I was aware of the need to not favour this conceptualisation of recovery above others.

My clinical experience as a healthcare worker and assistant psychologist also gave me an insight of what it is like being part of a team delivering mental health services. I feel like this grounding in service delivery gave me an understanding of the day-to-day running of mental health services, as well as the pressures, tensions and priorities that are prevalent in these contexts. This enabled me to consider the logistics of conducting research within the confines of a mental health inpatient service as time was going to be a limited resource, Due to this the project needed to be flexible to ensure as little disruption to the service as possible, and to ensure potential participants were aware of what the research was and why it was being conducted to ensure transparency throughout.

Being reflexive and reflective became extremely important during my research journey. During my PhD journey I learned that subjecting my own thoughts and conceptualisations of recovery and service delivery to critical analysis was essential. I employed strategies to minimise the risk of researcher bias such as engaging in regular supervision and writing a reflective research log.

I was drawn to RE (Pawson \& Tilley, 1997) for several reasons. Firstly, RE embraces that the fate of an intervention lies in the reasoning of participants (Pawson, 2013). My academic and clinical background meant I was aware of individual differences, the uniqueness of individuals and the varying responses to interventions. To see RE embrace these variations and seek explanations for these differences was very attractive to me as a researcher, particularly within the context of mental health. Secondly, my 
undergraduate and Masters training taught me that scientific data is often interlaced with ambiguity. RE draws upon the notion that the accumulation of evidence to support a claim can be falsified by new evidence in the future, thus meaning nothing is absolute truth (Popper, 1959); which aligned with my philosophical standpoint. Lastly, RE aims to include and explain confounding variables, rather than remove them, which lends itself to the complexities of real-life issues, such as mental health recovery.

In summary, I faced two new challenges when I started this PhD: being aware of my personal experiences of mental ill-health and recovery, and RE, both of which I was drawn to and both of which I have been lucky enough to enjoy learning about in my research journey.

In the next chapter, I present the methodology chapter which discusses the philosophical underpinnings of this project, the participants, data collection and data analysis. 
2 Methodology 


\subsection{Introduction}

In this chapter, I present the ontological and epistemological underpinnings of this project. I detail what realist evaluation (RE) is, and its logic of inquiry, in terms of context, mechanism and outcome. I provide a detailed account of what I did in terms of selecting and recruiting participants, how I collected and analysed the data and the ethical considerations relevant to this thesis. As the research evolved due to unexpected circumstances, the project was amended, therefore I will discuss the rationale for the amendment as well as what amendments were made.

\subsection{Realism}

How we think the social world is constituted, or what we think reality is (our ontology), shapes how we think we can know about our reality (the epistemology).

Realism is a broad logic of inquiry that is grounded in the philosophy of science and social science. It is not a method, but rather a methodological orientation; used for developing and selecting research methods. Realism is gaining increased attention as an alternative philosophy to positivism and interpretivism for evaluations in social sciences (Maxwell, 2008; Sayer, 2000).

\subsubsection{Ontology}

Ontology questions the form and nature of reality and therefore what can be known about it (Guba \& Lincoln, 1994). For realists, all human action is embedded within a wider range of social processes (Pawson \& Tilley, 1997); meaning even the most common actions are only understandable because they contain innate assumptions about a wider set of rules (Pawson \& Tilley, 1997). This means the real world and reality is complex. It consists of stratified layers of individual, group, institutional, and societal levels (Pawson \& Tilley, 1997). Realism, therefore, explains the real world through considering how social structures and contexts affect mechanisms, processes, and actions that lead to observable phenomena (see Figure 2). 
As reality is largely independent of our mind realist inquiry is the explanation of the underlying structures and mechanisms that generate a response in the subject matter. For realists, causation is not understood on the model of regular successions of events (Sayer, 2000; Pawson, 2006). Generative causation looks for causal powers within the objects, agents or structures under investigation (Pawson, 2006). Generative explanations hold that there is a real connection between events which we understand to be connected causally - one happening may well trigger another, but only if it is in the right conditions and in the right circumstances (Pawson \& Tilley, 1997). This means that it is not programmes that 'work', but the generative mechanisms that they release by way of providing reasons and resources to change behaviour' (Pawson \& Tilley, 1997, p. 36). Realist inquiry, therefore, aims to understand the conditions that are required for change to occur and to identify the causal mechanisms involved.

Our knowledge is not linear and is understood in terms of its location within different layers of social reality. Thus, unobservable structures cause observable events and reality can only be understood if we understand the structures that generate the observable events. Due to this there is a proposed layered and stratified ontology of the social world (Pawson \& Tilley, 1997) (see Figure 2). 


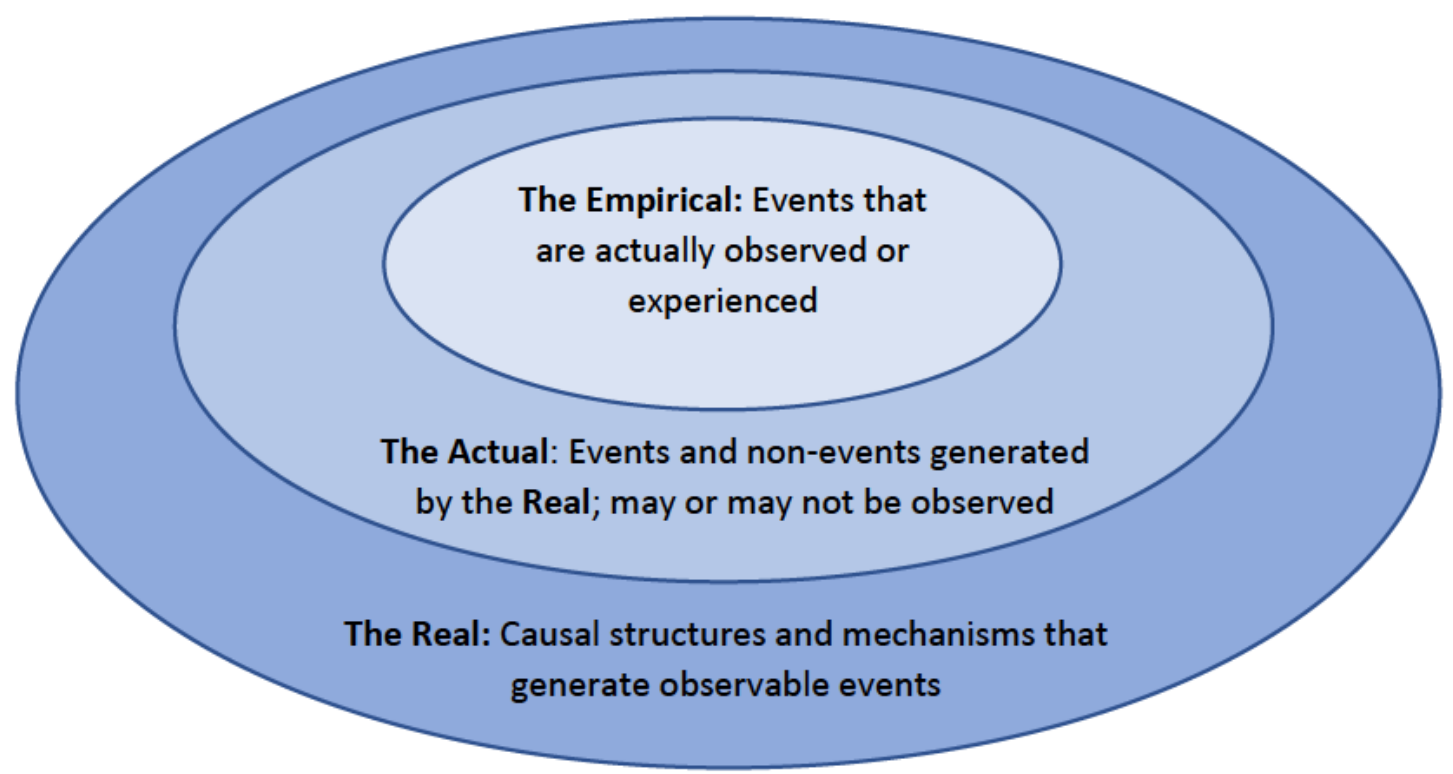

Figure 2 - Stratified Ontology within Realism

The first and most superficial layer is the 'empirical' which is what can be observed or experienced. Underneath the empirical level is the 'actual' which is what is going on that may not be observed but which is regulating the empirical. The final layer is the 'real'; this is the generative mechanisms that contribute to our understanding of the 'actual'.

\subsubsection{Epistemology}

Epistemology considers the relationship between the knower and what can be known (Guba \& Lincoln, 1994). Realism suggests that there is a real world in which people interact and they construct meaning in this world. There is a real world and that our knowledge of it is processed through human senses, brains, language and culture (Wong et al., 2012). Whilst these constructions may generate a valid understanding of the phenomena, it cannot and does not exhaust it. Reality exists independent of our understanding of it (ontology), and thus our knowledge is partial and contextual, meaning alternative constructions of the world are always possible (Altheide \& Johnson, 2011). For example, two individuals who experience mental health treatment may have differing reactions which is mediated by their understanding and experience. Therefore, what can be known about any phenomena is not definitive. 
The task of realist inquiry is to state theories about these underlying mechanisms. These theories are always fallible because direct access cannot be gained to test if they are true or not. There is an acknowledgement there can be more than one scientifically correct way to understand reality (Lakoff, 1987), as theories are grounded in a worldview, relative to place and time (Riege, 2003). For realists, facts are not universal truths but are conceived as theory-laden and conceptually relative (Searle, 1995).

Realist inquiry aims to produce true descriptions of the observable and unobservable world, whilst accepting that these descriptions are fallible, likely to only be approximately true and may prove erroneous.

\subsection{Realist Evaluation}

$\mathrm{RE}$ is a theory-based evaluation that aims to test and refine the theory that informed the development and implementation of the intervention being evaluated (Pawson \& Tilley, 1997). Theory-based evaluations are not only interested in measuring the outcomes of the intervention, but also in identifying the contexts and mechanisms that support these outcomes (Hansen, 2005). RE helps to explore what it was about an intervention that works, for whom, and in what circumstances by understanding the relationship between intervention participants and the intervention rather than evaluating the impact the intervention had on the person.

Tilley (1998) outlined three investigative areas that need to be addressed when evaluating an intervention using realism - context, mechanism and outcomes. I will now discuss each of these components separately.

\subsubsection{Context}

Context describes the pre-existing conditions that an intervention is embedded within (Marchal et al., 2012). It would be impossible to establish a direct, straightforward relationship between the intervention and outcomes without considering the contextual constraints the intervention sits within. According to realism, intervention recipients will only act upon the resources offered to them if they are within a conducive setting; 
therefore, the successes of an intervention are always influenced by the contextual conditions (Pawson \& Tilley, 1997; Pawson, 2013).

Within realism, context does not solely relate to the geographical location in which an intervention is implemented, but it considers interrelationships, social norms and values (Pawson \& Tilley, 1997). Pawson (2006) identified four levels that could influence the implementation and success of an intervention:

- Individual capabilities of key actors;

- Interpersonal relationships that develop in the locality within which the intervention is implemented;

- Institutional settings such as culture, rules, routines;

- Infrastructure and wider contexts such as national policies, guidelines, social rules.

These four categories were used to help me identify the contexts relevant to Woodlands, and helped to distinguish whether something was contextually, or mechanistically contributing to the service supporting individuals in their recovery. These categories also aligned with the ecological perspective guiding this thesis (see section 2.4.1), and enabled me to ensure that the stratified layers of social systems, central to realist ontology, were considered.

\subsubsection{Mechanisms}

Mechanisms capture the interaction of people with the intervention resources and 'pinpoint[s] the way in which the resources on offer may permeate into the reasoning of the subjects' (Pawson \& Tilley, 2004, p. 7). Investigating mechanisms allowed me to go further than asking whether a programme worked, to understand specifically what it was about Woodlands that led to an outcome pattern (Pawson \& Tilley, 1997). 


\subsubsection{Outcomes}

Outcomes were the intended or unintended consequences of the service offered by Woodlands that emerged from an interaction between the context and mechanism (Pawson \& Tilley, 1997).

\subsubsection{Context-Mechanism-Outcome Configuration (CMOC)}

A programme theory is a statement or a hypothesis about how an intervention is, or is not, working. It provides the logic, and often the assumptions that are made about the intervention, which includes the contexts and mechanisms that led to outcomes. This is presented in a context-mechanism-outcome configuration (CMOC).

Dalkin et al (2015) built upon the work of Pawson and Tilley (1997) and proposed an alternative form of the CMOC:

\section{Mechanism (Resources) + Context $\rightarrow$ Mechanism (Reasoning) = Outcome}

This revised $\mathrm{CMOC}$ acknowledges that intervention resources are introduced in a context, in a way that creates change in recipient's reasoning, leading to outcomes.

Explicitly disaggregating the components of the mechanism helped me address some of the challenges reported within other REs.

It has been reported that REs often emphasised either resource or reasoning, at the expense of the other (Pawson \& Manzano Santaella, 2012). By capturing both within the $\mathrm{CMOC}$, it meant that one component of the mechanism could not be favoured over the other. Secondly, there has also been variation within RE as to what constitutes a mechanism (Marchal et al., 2012). Some research has positioned mechanism as the processes responsible for change (Rycroft-Malone et al 2010; Ogrinc \& Batalden 2009), others considered mechanisms in terms of barriers and facilitators (Tolson et al., 2007), and Evans and Killoran (2000) simply identified the intervention as the mechanism. However, using the revised CMOC proposed by Dalkin et al (2015) I was able to adhere to Pawson and Tilley's (1997) conceptualisation of mechanisms as both reasoning and 
response. Thirdly, ambiguity has been reported when distinguishing between whether an element contributes mechanistically or contextually to the explanation of how the intervention works (Astbury, 2013; Williams et al., 2013; Dalkin et al., 2015). Byng et al (2005) stated that this ambiguity made it difficult to translate findings into a context, mechanism and outcome configuration (CMOC). However, using Dalkin's revised CMOC helped me to distinguish between what was contextually or mechanistically contributing to how the Woodlands service worked, as mechanisms were teased into two components. Dalkin et al's (2015) revised CMOC also provided a starting point when analysing the data, as I could start with identifying the key resources at Woodlands and begin working backwards and unpacking conducive or unconducive contexts, service-user or staff responses and expected or unexpected outcomes.

\subsubsection{Procedure}

$\mathrm{RE}$ is a set of principles as opposed to a set of prescriptive, methodological steps (RycroftMalone et al., 2010). RE is, therefore, not a strict technical procedure, but a general research strategy that requires creative innovation (Pawson, 2013). Pawson and Tilley (1997) identified three key stages of RE, which I have used to guide my approach to conducting the research reported in this thesis:

\section{- Stage One - Identification of Programme Theories}

The first stage involved the identification and generation of ideas about the context, mechanisms and outcome patterns that were important to Woodlands (Byng et al., 2005). This stage aimed to determine how, in theory, Woodlands was supposed to work in supporting individuals in their mental health recovery. This was achieved through conducting research interviews with individuals responsible for the design and development of Woodlands. I will refer to these individuals as programme architects (PA) for the remainder of this thesis. The data from stage one will be analysed to produce initial CMOCs of how Woodlands would work in supporting individuals in their recovery.

\section{- Stage Two - Testing the Programme Theories}

This stage involved consultations with other participant groups, these included intervention facilitators (staff), recipients (service-users) and after an amendment to the 
project design, commissioners. This stage tested the initial CMOCs from stage one and determined whether the theories about how Woodlands should work translated into practice.

\section{- Stage Three - Refining the Programme Theories}

The final stage of the RE was to refine and update the CMOCs relating to how Woodlands was supporting service-users in their recovery.

\subsubsection{Rationale for Realist Evaluation}

RE was considered the most appropriate framework for this project as it provided me with a grounding in how to consider the complexity of social interventions (Pawson \& Tilley, 1997). Using realist may also be beneficial in managing the tension in the diverging views of what ROC is which is an ongoing challenge in this research area. This was relevant when investigating the complexity often associated with contemporary healthcare systems and services. Greenhalgh et al (2015) proposes that the interventions associated with the health needs of modern society are complex, with multiple, interconnected components. Evaluating these types of interventions is therefore challenging. There are also multiple processes operating at individual, interpersonal, institutional and infrastructural levels, that influence human action (see section 2.3.1). RE is, therefore, designed to unpack the multiple, interconnected components, and stratified layers of reality to be able to consider how, why, for whom, and in what circumstances interventions work.

RE provides an approach for which the success of interventions depends on the response of individuals and the wider context in which these interactions occur (Wong et al., 2015). This enabled me to capture that what works for service-user A, may not work for serviceuser B, but also to explore reasons for these differences. This was particularly attractive when evaluating a mental health service, due to the individual nature of service-user's recovery journey. RE enabled me to steer away from the failed 'one-size-fits-all' approach to problems and interventions, and focusing solely upon characteristics of service-users to consider other contextual factors that can influence recovery.

This context-sensitive approach to evaluation may lead to context-sensitive solutions which account for the complex and dynamic systems operating in healthcare (Marchal et 
al., 2012; Greenhalgh et al., 2015). This was considered valuable for the area of mental health, and particularly ROC, as there have been numerous challenges to embedding ROC within inpatient settings. Therefore, using an approach which may lead to contextsensitive solutions is necessary to find practical ways of delivering ROC in these settings.

RE can also be applied in situations where gaining knowledge and insight about the workings of a programme is the aim, and where a programme is being implemented in a new context with no previous evidence of how it might work. This was advantageous for this project as there is currently limited knowledge of how ROC can be delivered successfully within inpatient settings (see Chapter 3 ), and because Woodlands is the UK's first charity run mental health inpatient service it has a unique context. RE appeared to be the most appropriate means of ascertaining and providing evidence of how Woodlands does, or does not work, as well as considering its unique context.

\subsection{Moving from Macro to the Micro}

In this section, I describe how I will use an ecological perspective to structure the overall thesis, as well as my analysis, so I consider the multiple connected levels that make up the contextual topography in which Woodlands operates.

\subsubsection{Bronfenbrenner's Ecological Systems Theory (EST)}

An ecological approach provides a framework that allows for the incorporation of the individual, their environment, and the interaction between the two. This therefore provides a crucial theoretical lens to investigate the contextual factors that are, or are not, conducive to ROC within inpatient contexts.

I will use Bronfenbrenner's $(1975 ; 1977)$ EST to guide the structure of this thesis and my approach to analysis so that the different levels of the system are considered. The theory states that human development is influenced by the different types of environmental systems, in the form of micro-, exo- and macro-level systems (see Figure 3). The theory, therefore, allows for the recognition of the broader interplay of factors beyond the person and demonstrates the interplay between, and interdependence among, the individual, immediate, indirect and social environments. Each of these ecological systems inevitably 
interact with and influence each other in all aspects of an individuals' lives. These systems include anything from political systems, health services, family and personality, which all work together to influence and affect health (Bronfenbrenner, 1994; Rosa \& Tudge, 2013; Tudge et al., 2016).

This thesis will categorise the different levels of the system Woodlands was operating within as follows (see Figure 3):

- Individual - this relates to the service-user accessing Woodlands.

- Microsystem - this contains relations between the service-user and the immediate environment surrounding them (Bronfenbrenner, 1977). In this thesis the microsystem was Woodlands and its approach to care. Interactions within the microsystem involved personal relationships with staff and other service-users and how these interactions influenced recovery progress.

- Exosystem - this level embraces the social structures which impinge upon the immediate settings in which service-users are found and as such influence what occurs in these settings (Bronfenbrenner, 1977). In this thesis, the exosystem relates to the mental health referrals system that operates, as well as external agencies, such as education providers, who influenced whether individuals could access certain community-based opportunities.

- Macrosystem - this consists of the blueprints of a society, such as unwritten rules, norms, laws and regulations (Bronfenbrenner, 1978). In this thesis, the macrosystem relates to societal attitudes and social determinants of mental health. 


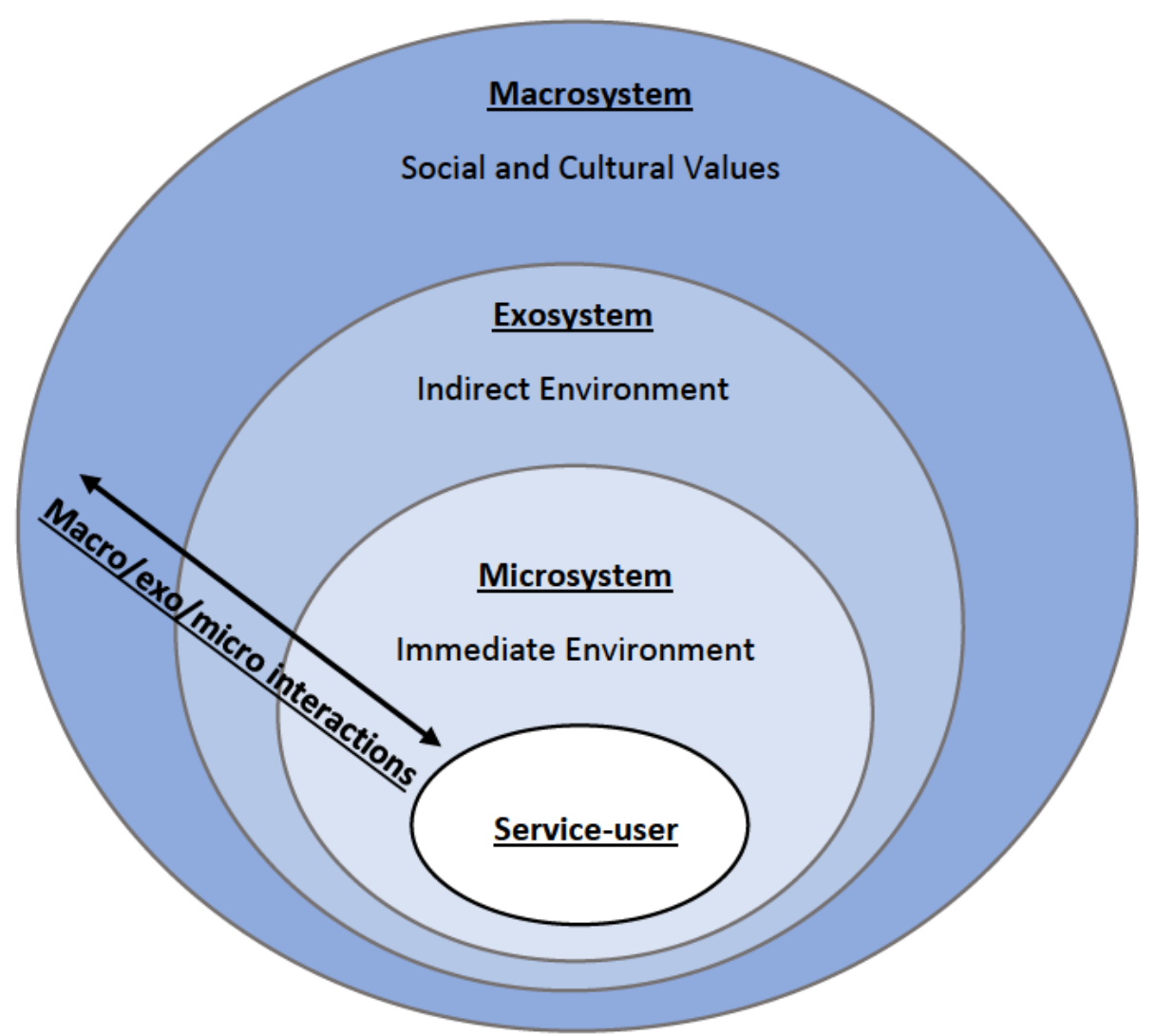

Figure 3 - The Levels of Bronfenbrenner's Ecological Systems Theory

The EST (1977) will inform the overall structure of the findings presented within this thesis.

In Chapter 4, I present my analysis of the commissioning and referrals process within Wales (the exosystem). I argue that Woodlands encountered challenges due to being a new provider of inpatient care and attempting to provide an alternative model of care for recovery (see Chapter 4). I also argue that numerous system-level factors meant that there was a limited need for a locked service providing recovery in the current market.

I then proceed to present my analysis of the microsystem of Woodlands in the form of the staffing model (see Chapter 5) and how service-user choice and responsibility was supported by the service (see Chapter 6). In Chapter 5,1 argue that the exosystem of the referrals process influenced how the staffing levels worked at Woodlands. In Chapter 6, I argue that the macrosystem of wider society and experiences of rejection and stigma influenced how Woodlands operated in terms of supporting individuals in acting upon their choices. These chapters both highlight that the that the stratified layers of social systems were particularly influential to the resources at the microsystem of Woodlands. 
I also consider the interaction between, and within, these systems. This will mean that the different levels of the system will be discussed within each chapter to allow me to consider how these different levels interacted and influenced individual's recovery at Woodlands. This will ensure that the interactions between the different systems are considered both within and between the findings chapters. The decision to structure the qualitative findings in this way was made so that I could focus on the broader aspects of the context and then become more specific as the finding's chapters progressed. Discussion of the individual and macro elements are interwoven into Chapters 4, 5 and 6.

\subsubsection{Compatibility of Realist Evaluation and Ecological Systems Theory}

Ecological perspectives have been increasingly used in public health and health promotion literature (Porta \& Alvarez-Dardet, 1998; Susser, 1998). The use of ecological thinking within public health acknowledges the complexity of public health problems and interventions and construes causality as context dependent (Koopman \& Longini, 1994). An ecological perspective considers context-level variables which surround the variable under investigation alongside variables that operate at the individual-level (Diez Roux, 2001). This interest and focus on context aligns with one of the chief concerns of RE.

Realist philosophy understands 'reality as comprising multiple, nested, open systems in which change is generative, context dependent and time irreversible' (Westhorp, 2012, p. 406). Westhorp $(2012 ; 2013)$ advocates for realist evaluators to understand different levels of systems to conceptualise and analyse the multiple processes of causation that contribute to outcomes. RE identified four levels that could influence the implementation and success of an intervention (Pawson \& Tilley, 1997; Pawson, 2006):

- Individual capabilities of key actors;

- Interpersonal relationships that develop in the locality within which the intervention is implemented;

- Institutional settings such as culture, rules, routines;

- Infrastructure and wider contexts such as national policies, guidelines, social rules. 
These align with the individual, micro, exo and macrosystems promoted in the EST (Bronfenbrenner, 1975; 1977). I therefore considered Bronfenbrenner's (1977) EST to be a complimentary theory to ensure that the multiple layers of reality are considered when evaluating how Woodlands operates within these open systems.

I note that Bronfenbrenner developed his EST theory and conceptualised this as the Process-Person-Context-Time (PPCT) model (Bronfenbrenner \& Evans, 2002). Bronfenbrenner and Evans (2002) reported that the context component of the new PPCT model was underpinned by the EST. As I was using the EST to focus upon the context in which Woodlands operated, and to capture the multiple layers of reality, the addition of process, person and time would have been a deviation from the focus of this evaluation. For this reason, I decided to use Bronfenbrenner's earlier work on the EST to focus upon the micro, exo and macrosystems that were relevant to Woodlands as this is very similar to how Pawson and Tilley (1997) conceptualised context within RE.

\subsection{Research Participants and the Project}

The specific form of relationship a prospective investigator has or will develop with the people and/or setting of interest generates a set of challenges in the process of gaining entry, which often involves some negotiation (Lofland et al., 2006). Although I was in the position of the 'outside' participant researcher role (Lofland et al., 2006), negotiating access to Woodlands was aided by the fact that the service approached Swansea University and part-funded the PhD studentship. This meant that there was already an established link between myself and the service. As it is typical for an outside researcher to gain acess to persons or settings through an already established connection, I was able to use this connection to identify a gatekeeper who could support access to the service and potential participants. During the negotiations regarding gaining and maintaining access to the service certain factors were decided:

- Contact with the service could be via telephone or email and did not always need to include the gatekeeper (for example when arranging interviews with staff members to maintain anonymity); 
- Access to the service at times that were convenient with service-users and staff, for example not conducting interviews at times when service-users had prearranged leave or would mean they had to end their leave prematurely and did not clash with therapeutic interventions inside or outside of the service, the daily morning meeting or clinical team meetings;

- Provide the service with sufficient notice of when the interview was being conducted so that they could factor this in with the staff rota so that the service was not short-staffed;

- I made myself aware of the visitor policies, for example signing the vistor book when arriving at Woodlands, call the service so that interview dates and times were included within the diary, wearing my university name badge, and informing staff of when and where I was when at the service;

- I maintained contact with the gatekeeper throughout the lifetime of the project, either via email or in person, and would invite the gatekeeper to attend supervisory meetings during the development of the project design;

- I discussed service-user capacity and any safeguarding or safety issues with the clinical team prior to the interview to maintain researcher, service-user and staff safety - this was not extended to staff interviews;

- I attended some morning meetings so I could introduce myself to potential participants and explain about the project within the meeting, but also have discussions individually with service-users if necessary;

- It was agreed that I would be provided access to an anonymised database that contained the routinely collected quantitative data at the end of the data collection period, which was December 2019. This would be provided on a password protected USB stick that I supplied and then the data was transferred to a password protected university computer. 
The gatekeeper to the service was advantageous in the negotiating access to Woodlands and potential participants.

\subsubsection{Gatekeeper}

Gatekeepers have been generally recognised as playing an important role in social research (de Laine, 2000) as they can assist in gaining access to the research field, the organisation and potential participants (Crowhurst, 2013).

The advantages of using a gatekeeper in this project were to: speed up the recruitment process (de Laine, 2000); help identify individuals who fulfilled the criteria as PAs; to act as a guarantor for my legitimacy as a researcher (Whyte, 1993); and to inform the strategic planning for realistic and achievable recruitment and data collection based on their knowledge of Woodlands. These benefits had the overarching aim of ultimately saving me time throughout the project. For this project, the Development Consultant, who oversaw the delivery and development of Woodlands, acted as the gatekeeper.

I was aware that by using a gatekeeper the selection of potential participants could be biased as a means of protecting the gatekeeper's own interests and those of Woodlands (Emmel et al., 2007). To address this, I provided the gatekeeper with specific inclusion and exclusion criteria for potential participants, which I hoped would help mitigate any potential biases.

I was also mindful that the gatekeeper held a senior position at Woodlands, and I wanted to make sure that their authority did not overrule the autonomy of potential participants to refuse to participate (Wanat, 2008). I regularly reminded the gatekeeper that although their role was to identify potential participants, the ultimate decision of participation rested with the individual being approached and I emphasised that all participants could withdraw at any point. The gatekeeper was not informed of who did or did not participate in the research and pseudonyms were used when referring to individuals when reporting the findings. 


\subsubsection{Participant Selection}

Pawson and Tilley (1997) recommend that participant selection should be based upon their 'CMO investigation potential'. I informed the gatekeeper of the need to identify an appropriate participant sample who could provide information about context, mechanisms and/or outcomes relevant to Woodlands (Greenhalgh et al., 2017). The following criteria were provided to support the gatekeeper in their identification of participants.

\subsubsection{Programme Architects (PA)}

I considered that PAs would be able to provide rich causal insights to help develop initial CMOCs that would be tested within practice at Woodlands. Due to this the following inclusion criterion was developed:

- Was involved in the development and design of Woodlands;

- Still worked at the organisation;

- Consented to participate.

\subsubsection{Staff}

Pawson \& Tilley (1997) suggested that staff have a broad range of experience relating to intervention successes and failures and hold specific ideas on what works within an intervention and who it works for. As professionals have the experience of working with a range of people, they will be able to provide an account of contexts and mechanisms. I considered that staff would also be able to provide information about conducive and nonconducive contexts, barriers, facilitators and outcomes of Woodlands. The following inclusion criterion was therefore developed for staff:

- Played an active role in service delivery or monitoring of Woodlands;

- Still worked at Woodlands;

- Consented to participate. 


\subsubsection{Service-users}

The intervention recipients were service-users accessing Woodlands. Key authors have stated that intervention recipients are less proficient at identifying contexts and mechanisms of interventions, as they only have their own idiosyncratic experiences to draw from (Pawson \& Tilley, 1997). Despite this, I believed that service-users may be able to discuss their response to resources at Woodlands, and outcomes. All service-users were given the opportunity to participate, unless they met any of the exclusion criteria:

- Unable to speak English;

- Unable to provide consent for themselves as outlined in the Mental Capacity Act (MCA) (2005).

\subsubsection{Carers}

Involving carers has become a central tenet of contemporary psychiatric policy (Coulter \& Collins 2011). However, carer involvement in clinical practice and research has resulted in barriers, such as feeling excluded from the treatment process and power imbalances between carers and staff (Askey et al., 2009; Cree et al., 2015). Due to these noted difficulties I put in provisions to try and make carer involvement in the project as easy and accessible as possible.

I was flexible as to when interviews were conducted, such as scheduling interviews for the same day they were at Woodlands, offering telephone interviews and ensuring the interviews were not overly time-consuming. The following inclusion criterion was used for carer participants:

- The service-user had nominated and consented to their carer being contacted;

- The carer was over the age of 18;

- The carer consented to participate.

\subsubsection{Recruitment Process}

The recruitment process differed between the individual participant groups for this thesis. 


\subsubsection{Programme Architects and Staff}

The gatekeeper identified and approached all the individuals that could be potential PA or staff participants. The gatekeeper asked these individuals if I could contact them directly using their work email address. All potential PA and staff participants agreed for me to contact them on their work email. I sent these individuals an email which included the participant information sheet (PIS) (see Appendix A) and provided them with the options of either contacting me via email with questions, declining to participate, or to arrange an interview.

All PA and staff interviews were conducted face-to-face. PAs were given the option of the interview being conducted at the organisation's Head Office, or at Swansea University in a private conference room. All staff interviews were conducted in the private family room at Woodlands when the staff member was on shift to reduce any potential burden to the staff member, clinical team or service delivery at Woodlands. All PA and staff participants completed consent forms (see Appendix B) and were given debrief sheets at the end of their interviews (see Appendix C).

\subsubsection{Service-users}

The gatekeeper made initial contact with all service-users at Woodlands and provided them with a letter inviting them to a briefing session to be held at Woodlands, (see Appendix D). The briefing session was designed as an opportunity for me to introduce myself, provide a project overview and answer any questions. The briefing session was not compulsory and if individuals did not attend, or were unable to attend the briefing session, this did not impact their ability to participate.

The briefing session was poorly attended - the reasons for this were low service-user occupancy and individuals being on leave or asleep at the time of the meeting. Due to this information about the research was instead delivered in a one-to-one conversation between myself and the service-user, at a time convenient to them. During these one-toones I provided service-users with a written PIS, (see Appendix E) and went through this with them verbally. All potential participants were given time to consider whether they wished to participate and could change their mind at any time. 
Service-users could express their interest to the clinical team, post a note in a sealed and confidential box I placed at Woodlands or talk to me in person when I was at the service. If they consented to participate, then an interview date and time was arranged that was convenient with them. All service-user research interviews were conducted in the private family room at Woodlands. All service-user participants completed consent forms (see Appendix F) and were given debrief sheets at the end of their interviews (see Appendix G).

\subsubsection{Carers}

Carers were nominated by service-users at Woodlands (see Appendix $\mathrm{H}$ ). If consent was given, carers were sent a letter informing them of the project and how they could express interest in participating in the project, (see Appendix I), and a participant information sheet (see Appendix J). Carers would complete a consent form (see Appendix K) and be given debrief sheets at the end of their interviews (see Appendix $L$ ).

\subsection{Data Collection}

In this section I provide an overview of the data collection methods used within this project.

\subsubsection{Realist Interviews}

The purpose of the realist interviews was to inspire, validate, falsify and modify hypotheses about how Woodlands worked and to build knowledge of what happened in a natural setting (Pawson, 1996). Realism is based upon the notion that 'nothing works unconditionally in all circumstances' (Tilley, 2000 p. 126), therefore questioning within a realist interview should allow for heterogeneity to emerge. The objective of these interviews was to elicit the intervention's story, which was achieved by capturing participants' experiences that highlighted the processes of change and outcomes that occurred (Patton, 2003).

I noted that there appeared to be little guidance on how to operationalise the principles of realism in an interview, and how researchers conducted realist interviews. Some 
researchers, such as Etheridge et al (2013) simply stated that their topic guide was based upon the principles of RE but provided no explanation of how they achieved this. I used the Manzano's (2016) and Westhorp \& Manzano (2017) papers to inform the development of my topic guides and how to influence my questions based upon realist principles. Topic guides were developed for each participant group (PAs, staff and serviceusers) as the focus of the interview changed for each group (Dalkin et al., 2015), (see Appendix M, Appendix N, Appendix O and Appendix P).

I also reflected upon my experiences of conducting realist interviews to provide transparency of how I maintained realist underpinnings throughout.

\subsubsection{Teacher-Learner Cycle}

Realist interviews adopt a teacher-learner cycle. Researchers are expected to take an active role in directing the questioning and conversation, so it remains on the specific topic under evaluation (Manzano, 2016), but the role of teacher and learner are interchangeable between the participant and researcher. This meant I needed to give participants time and space to share their own understanding and experience of how the intervention worked in practice, as well as teach the interviewee the initial programme theories derived from literature and interviews with PAs.

This interview approach differed from my previous interview experience where I felt I had to deliberatively act naive to avoid data contamination or influencing participant's narratives. I was aware of the potential criticism that conducting an interview using a teacher-learner cycle could have, such as leading participants, therefore I tried to put processes in place to manage this during data collection.

Initially I would leave space for participants to share their understanding and experience. This allowed for participants to not be influenced by me presenting alternative theories or experiences. Once the participant had shared their understanding, I would then present alternative theories or experiences. This created further discussion, and often participants shared their perspective towards the alternative theories. 
If participants had difficulties answering the realist-informed questions relating to how, why, for whom or in what circumstances Woodlands worked supporting individuals in their mental health recovery, I would use some of the following phrasing:

- "I see from the brochure for Woodlands that you aim to do... but from being at the service it appears that..." (with the aim of referencing my observations)

- "I have read some studies that say..." (with the aim of referencing literature)

- "In some other interviews I have conducted they thought this... is this similar to your experience..." (with the aim of referencing other participant responses)

When I presented information based upon my observations, literature or previous participant data there was often agreement with what I had proposed. I would then ask participants if they could expand upon this using their own words and experiences of Woodlands. By using these types of phrases, I wanted to prevent participants from feeling pressured to answer in a certain way, or that there were right or wrong responses. I hoped this phrasing would also mean I was not attaching my own understanding to the programme theories being presented, which I hoped would prevent participants feeling there was a right or wrong response. I also achieved this by using terms such as 'some people think it works by', 'whereas others think this', to show there can be more than one understanding of the intervention. In interviews where I was using this phrasing more regularly, I found myself adopting more of the teacher role and was unable to strike a balance between being the teacher and learner roles. I found this was typically occurring in service-user interviews.

\subsubsection{Audio Recording and Transcription}

At the beginning of the interviews I asked all participants whether they consented to me audio recording the interview, and no concerns were raised. I recorded all interviews using a digital audio recorder. I informed participants verbally when the audio recorder was on, and then again when it was turned off. Following each interview, I downloaded the audio 
file onto a password protected computer at the university for transcription and deleted the audio file off the recorder.

It has been widely acknowledged that transcription is time-consuming (Hammersley \& Atkinson, 2007) but I found it provided me with an opportunity to reflect not only upon the data but also on my interview technique. This was beneficial given that I was new to realist interviews and struggled with finding the balance between teacher and learner. I decided to transcribe the data as soon as I completed each interview, so I could use any knowledge learnt in future interviews.

Throughout all the qualitative findings chapters I have used the following the notations. (.) to represent when a participation paused, and ... to represent where I have omitted some parts of their speech with the data extracts presented.

\subsubsection{Quantitative Outcome Measures}

Woodlands provided me with permission to access their routinely collected data via a centralised and anonymised database, which formed part of the discussions relating to negotiating access to the service. This access to data was agreed with the gatekeeper and data collection would end on the $31^{\text {st }}$ December 2019. This date was agreed to allow sufficient time for analysis and the write up of the results. In this section, I will detail the agreed protocol for this access and will provide the psychometric properties for the measures used within this thesis.

\subsubsection{Agreed Protocol for Routinely Collected Data}

As the quantitative data was routinely collected at Woodlands I was not involved in the collection of this data. The agreed protocol was that the ReQoL-10 (Keetharuth et al., 2017) and CORE-OM (Evans et al., 2002) data would be collected upon admission, and every month thereafter, and the VOICE (Evans et al., 2012) measure would be collected one month into admission and every 6 months thereafter. I would then be given electronic access to an anonymised database where I could analyse for changes over time. I was given access to the routinely collected data electronically by a senior nurse at Woodlands who was responsible for the management of the database. The database was 
electronically transferred to a password protected university computer for analysis using an encrypted USB stick.

In the following section I will review the psychometric properties of each measure, which show that the measures were valid and reliable for use within the research.

\subsubsection{Recovering Quality of Life - 10 (ReQoL-10)}

The ReQoL-10 (Keetharuth et al., 2017) was developed to assess the quality of life (QoL) of people with different mental health conditions (see Appendix Q). It offers a brief 10item patient reported outcome measure focusing on the process of recovery for serviceusers. The questions covered areas of QoL that service-users identified as important. These were, activities (meaningful), belonging and relationships, choice, control and autonomy, hope, self-perception, well-being and physical health.

Scoring - Each question was scored on a 5-item scale from 0 'none of the time' to 4 'most or all of the time'. An overall score was calculated by summing the numbers for each question, which meant scores could range between 0-40; with a higher score indicating better QoL.

Reliability - The ReQoL-10 has reported good internal reliability $(\alpha=0.85)$, and test-retest reliability $(\rho=0.70)$ in both the general population and a clinical sample (Keetharuth et al., 2018).

Validity-As the development of the measure was informed by service-user perspectives, the domains captured in the measure were considered to have high face validity and content validity. The ReQoL-10 measure has previously reported good convergent validity with the Short Warwick-Edinburgh Mental Well-Being Scale (Tennant et al., 2007) and the Clinical Outcome in Routine Evaluation-10 (Keetharuth et al., 2018), which are used in research and practice.

\subsubsection{Views on Inpatient Care (VOICE)}

The VOICE (Evans et al., 2012) measures service-user's perspectives of therapeutic contact, care, trust and respect they receive from staff (see Appendix R). The VOICE is a 19-item measure that was developed using a participatory methodology to ensure 
service-user involvement throughout the design and development of the measure. Repeated focus groups were conducted, and this data was thematically analysed by service-user researchers to draft a measure which was further refined in service-user consultations. The focus groups included a broad range of ethnicities, diagnoses and ages. The items in the measure relate to admission, care and treatment, medication, staff, therapy and activities, the environment and diversity.

Scoring - Individual item scores range from 1 'strongly agree' to 6 'strongly disagree'. An overall total was obtained by summing up the individual item scores, with the possible total scores ranging from 19 to 114; higher scores indicated more negative perceptions of the inpatient facility and care.

Reliability - The internal consistency of the scale has been reported as high $(\alpha=0.92)$ (Evans et al., 2012), which suggests the items measure the same underlying construct. The test-retest reliability has also been reported as high $(\rho=0.88)$ (Evans et al., 2012).

Validity - As the measure was informed by service-users, the domains were considered to have high face and content validity. A significant association has been reported between the VOICE and the Service Satisfaction Scale (SSS) (Greenfield \& Attkisson, 1989; 2004) which evaluates residential settings for individuals with serious mental illness, indicating high convergent validity (Evans et al., 2012).

\subsubsection{Clinical Outcomes in Routine Evaluation Outcome Measure (CORE-OM)}

The CORE-OM (Evans et al., 2002), a 34-item scale, was developed to assess the efficacy and effectiveness of therapeutic input in relation to service-user distress (see Appendix S). The items covered four domains; subjective well-being, problems/symptoms, life functioning and risk. Well-being depicts the affective tone, and the QoL of the serviceuser. Problems/symptoms relate to symptoms of anxiety and depression, the aftermath of trauma, and physical correlates of psychological health. Functioning relates to functioning in daily life as well as in social and close relations. Risk covers self-harm and suicidal ideation, as well as threats of violence, and perpetrated violence against others.

Scoring - Each of the 34 items were scored on a 5-point scale ranging from 0 'not at all' to 4 'most of the time'. Scores were reversed for the positively framed items. A total score 
was calculated by summing up all the items, dividing by 34 (the total number of items) and $\times 10$ to provide a number on the $10-40$ scale. A higher score indicated higher psychological distress, and the clinical cut-off point when using the 10-40 scale is a score of 10. Cut-offs are statistically derived points which differentiate between what are known as clinical and non-clinical populations. If a service-user scores above 10 on the CORE-OM, their score is said to be more representative of a clinical population. These population norms were derived from large samples of service-users receiving therapy or mental health intervention, and wider population samples.

Reliability - The internal consistency of the measure has reportedly ranged between $(\alpha=$ $0.75-0.95)$ and the test-retest reliability was classified as excellent (Evans et al., 2002).

Validity - When compared to other conceptually related measures, such as the General Health Questionnaire (Goldberg, 1972), Symptom Checklist-90 (Derogatis, 1992) and the Becks Anxiety Inventory (Beck et al., 1988), good convergent validity has been reported (Evans et al., 2002). When compared to clinicians' ratings of service-user risk good convergent validity has also been reported (Evans et al., 2002).

\subsection{Data Analysis}

\subsubsection{Framework Approach (Qualitative Analysis)}

Framework approach (Ritchie \& Spencer, 1994) was used to guide data analysis of programme architect, staff and service-user research interview data (see Figure 4).

The initial stage involved immersing myself in the PA data through the processes of transcribing, reading, rereading and writing notes about initial themes. The development of the framework was based upon the data from the PAs, as this would allow for data to be compared with staff and service-user accounts. At this stage, an inductive approach was adopted, which allowed for descriptive categories of data to be identified from within the dataset.

Information specific to the resources of Woodlands and how these were intended to support service-users in their recovery were recorded in NVivo. I highlighted each 
separate location when a key resource of Woodlands was identified to provide me with a point of reference for all the relevant sources within the dataset which related to that specific resource. The categories from the PA data were then used to develop a coding framework, which was used to analyse staff and service-user transcripts (see Appendix T). The coding framework was based upon resources (mechanism) that PAs identified as important at Woodlands to support individuals in their recovery. Staff and service-user data was analysed using the coding framework developed in stage 2 from the PA data (see Figure 4). The framework was piloted on two staff transcripts, and 1 service-user transcript to see if the framework categories required refinement, which was not necessary. This was mainly due to the staff and service-user interviews being tailored to focus upon the areas of service delivery that PA identified as important to how Woodlands supported individuals in their recovery.

Once all the data was coded using the framework developed in stage 2 these individual data extracts were reviewed again to identify data that related to the context, reasoning (mechanism) and outcomes for each specific resource. I found identifying the resources (mechanism) first, allowed for easier identification of context, reasoning and outcomes. This process relied upon my judgment and inferences; therefore, it is possible that a different analyser could have identified different aspects. To address this, I engaged in discussion with the supervisory team who provided feedback with regards to my data analysis.

All data extracts that provided rich causal insights into the workings of Woodlands were considered for their inclusion within the write-up of this thesis. The criteria used to determine if a data extract provided rich causal insights into the contexts, mechanisms and outcomes relevant to Woodlands was informed by the philosophical principles of realism and the RAMESES standards (Greenhalgh et al., 2016) (see Appendix U).

The process whereby I systematically applied the framework to all the data, involved my judgement about the meaning and significance of the data. Judgement is, however, transparent within the framework approach, and therefore adds to the replicability and robustness of the analysis. 


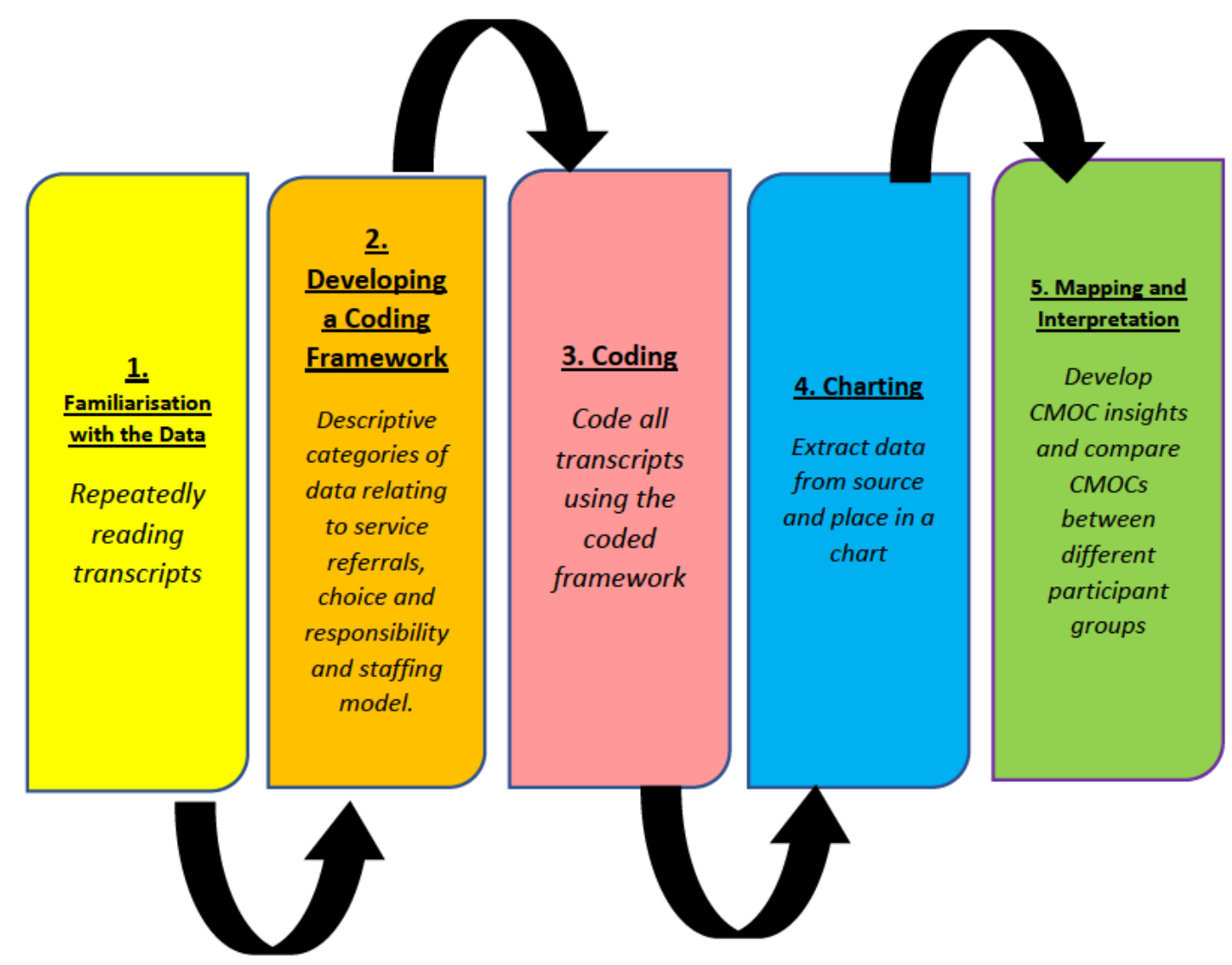

Figure 4 - Adapted Framework Analysis Process (Ritchie \& Spencer, 1994)

\subsubsection{Using NVivo}

As stated, NVivo was used throughout my qualitative data analysis. Electronic techniques of data management and coding, such as NVivo, have been argued to ensure 'that the user is working more methodically, more thoroughly, more attentively' (Bazeley \& Jackson 2013 p. 3). NVivo allows for assigning multiple categories to the same chunk of data (Hilal \& Alabri, 2013), which was particularly useful for this project and the challenge of determining whether something was contextually or mechanistically contributing to the intervention.

I was mindful that I had not used NVivo before and therefore had to factor in time to learn it, however I considered this a necessary use of my time to manage the dataset. Additionally, I had read that some researchers had expressed caution with the use of software programmes as researchers failed to interpret the data adequately and instead just code the data (Denzin \& Lincoln, 2005). To avoid this, I also used flipchart paper to 
form links and associations between the categories and to move from coding to interpretation and used the writing up process as part of the data analysis process. I found through the writing up process that I could begin to see categories within the data and could really begin to understand and link what different participants were saying about a topic of interest. At this stage I was also able to position what participants were suggesting about service delivery alongside the current literature.

\subsubsection{Quantitative Analysis}

The quantitative data was analysed using a case study approach. This enabled me to document changes to service-user ratings over time and identify patterns across and between service-user participants. It should be noted that I am aware that data derived from a single subject requires interpretive caution; thus, I do not attempt to offer firm conclusions based on my data. Rather I emphasise the suggestive implications of my results, see Chapter 7. Due to the unforeseen problems with data collection (see section 7.1), I focused my analysis on the individual items of the measures, as opposed to the overall total score to identify patterns in this data.

As I collected both qualitative and quantitative data, I needed to integrate the data therefore in the next section I discuss how I attempted to do this.

\subsubsection{Method Triangulation}

Method triangulation was considered appropriate as RE by nature is method neutral (Pawson \& Tilley, 1997). Realism does however value the use of mixed methods and states the selection of how to collect data should be based on how informative it is for the study. The perception that the principles of quantitative and qualitative methods are distinct, competing and separate paradigms, has resulted in some feeling these differences mean the paradigms are incommensurable (Kuhn, 1970). However, my view is that any concerns relating to the incommensurability of philosophical positions, can be set aside if the mixing of methods effectively addresses the research question. Furthermore, Rogers (2008) stated that complex social issues, such as those seen within healthcare, tend to be unforgiving to rigid, inflexible researchers who insist on an epistemological stance that ignores the realities of practice. If presented clearly, mixed methods can hold an 
important place in progressing knowledge and enable researchers to adequately address the complex, multifaceted issues of social interventions (Raven et al., 2011). For this project I used method triangulation in an attempt to integrate my two datasets (see Figure $5)$.

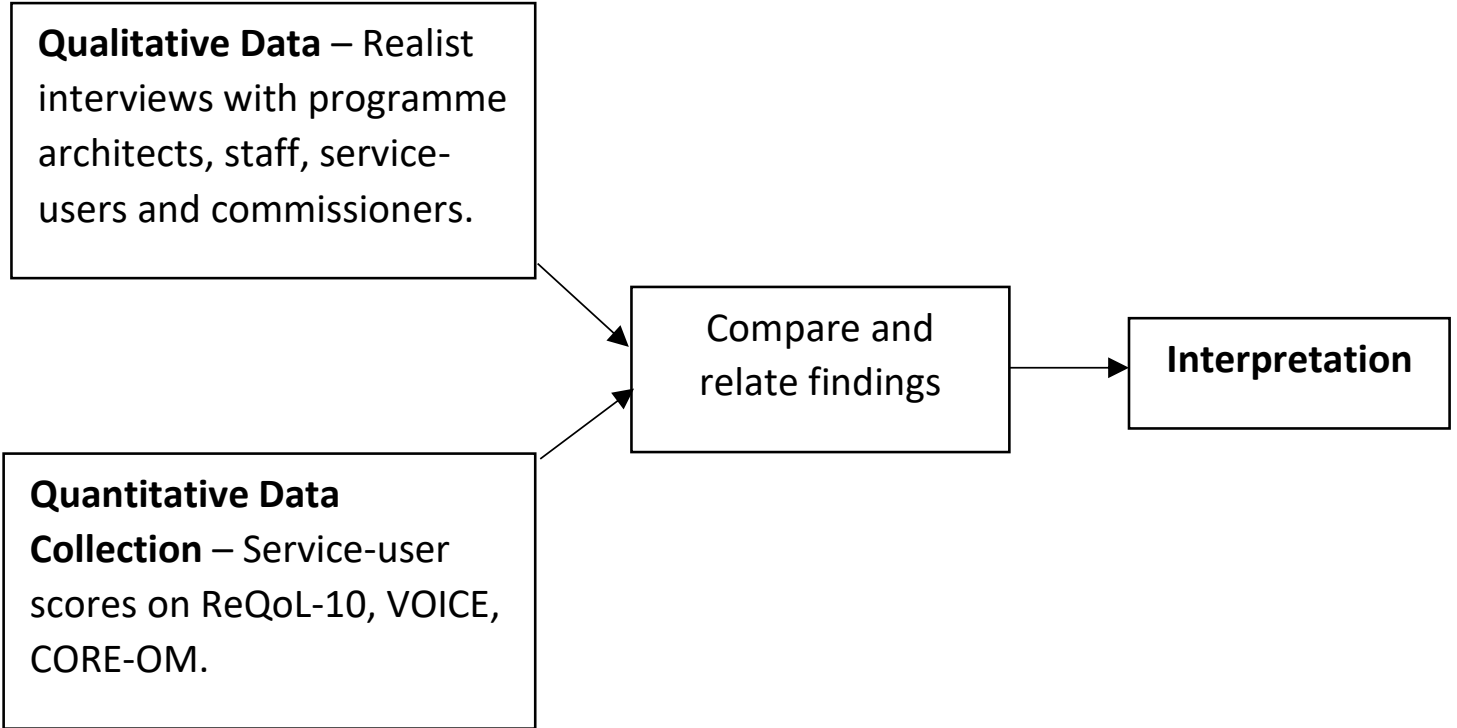

Figure 5 - Method Triangulation Process

\subsubsection{Triangulation Protocol}

A triangulation protocol (Farmer et al., 2006) was applied to interpret and integrate the qualitative findings from programme architect, staff, service-user and commissioner interviews. The findings from the qualitative chapters were then interpreted and integrated with the results from the routinely collected quantitative data in order to identify areas of convergence, divergence and complimentary findings between the datasets. I triangulated the data using a convergence coding scheme:

- 'Agreement' indicated that the key finding was identified across the participant groups, or across both the methods;

- 'Partial agreement' meant the finding was partially covered across the participant groups, or across both methods;

- 'Disagreement' evidenced contradictory findings across the participant groups, or between the two methods. 
- 'Not Addressed' meant that the qualitative or quantitative data did not cover this specific research area.

See Appendix $V$ for how I used this protocol specifically for the data from this project.

\subsection{Ethical Considerations}

Socially sensitive research has been defined as 'research which potentially poses a substantial threat to those who are or have been involved in it' (Lee, 1993, p. 4). Participants could potentially interpret the research as a threat as there were potential social consequences or implications for some participants (Sieber \& Stanley, 1988; Lee \& Renzetti, 1993); such as staff talking about their current employer, or service-users talking about their current treatment provider. Due to this several ethical considerations where necessary for this project. In this section, I will outline the ethical considerations relevant to this project.

\subsubsection{Ethical Approvals}

The National Standards for Public Involvement in Research (2019) suggest that research should provide opportunities for inclusion, working together to build and maintain productive relationships, promotes support and learning that builds confidence and skills for those involved, provides two-way targeted communication, drives improvement in health care research, and protects the public interest. However, one limitation of these standards is they fall short when it comes to answering critical questions relating to the rationale behind the emerging involvement imperative, such as why involvement should be undertaken in the first place (McCoy et al., 2018).

Public involvement in research is seen as a marker of good research practice because it can lead to better designed and relevant research, with clearer outcomes (National Institute for Health Research, 2016). Other positive impacts are reported, including an increased understanding and insight of researchers in their field, an increased sense of worth and skills gained as a result of involvement, and enhancing its quality and appropriateness (Brett et al., 2014; Thompson et al., 2014). 
Due to this, I sought advice from the Patient Experience and Evaluation in Research (PEER) Group at Swansea University to ensure the research was suitable and accessible and to ensure there was service-user and carer involvement within the design and development of the research project.

Ethical approval was sought and approved by Swansea University's Ethics Committee (Reference 010818a) and NHS Wales Ethics Committee REC 6 (Reference 18/WA/0315) (see Appendix W and Appendix X).

\subsubsection{Confidentiality and Anonymity}

I made attempts to ensure no individuals were identifiable by removing any personal information and using pseudonyms throughout. I used a pseudonym for the service; however, I am aware that the organisation and service could be identifiable based on the uniqueness of the service provider, and therefore anonymity could not be guaranteed (Kaiser, 2009). Woodlands were made aware that anonymity could not be guaranteed and accepted that the only provision available to me was to use a pseudonym. All pseudonyms within this thesis were selected at random by the researcher. The quantitative dataset was already anonymised before it was made available to me.

My approach was to maintain confidentiality throughout except if a participant disclosed information relating to a risk to self, others, absconding or details of poor practice. If this occurred Woodlands would have been informed, and if appropriate, the Health Care Inspectorate Wales, police and/or local authority would have been contacted. All participants were aware of this at the beginning of the project and reminded of this at the beginning of all interactions with the researcher, as well as before the interview to maintain transparency.

\subsubsection{General Data Protection Regulation (GDPR)}

I adhered to GDPR throughout the project and all the paperwork used was designed using GDPR guidance. All electronic information was stored on a password-protected system, which was only accessible by the researcher. All paper copies were stored in a locked cabinet. Following transcription, all audio recordings were deleted from the recording 
device and stored on a password-protected university computer. All data will be destroyed within five years upon completion of the project or following publication, except for the original copy of the routinely collected data which is stored and belongs to Woodlands. I will however delete the anonymised version of the quantitative database provided to me within five years of completing the project.

All participants were given the right to withdraw from the study. If a participant requested removal from the study then I would have safely destroyed any relevant data and removed these from any datasets, however, this did not arise in this project.

\subsubsection{Informed Consent}

Within research, specific legal requirements, outlined within the MCA (2005), apply to adults whom 'lack capacity to make decisions because of an impairment, mental disorder or disturbance in the functioning of the mind and brain'. The MCA (2005) states that researchers should assume that all persons have the capacity to make decisions unless there is proof to suggest otherwise. To address any potential issues of capacity and as part of my negotiated access to the service, I was required to ask the clinical team to raise any concerns regarding capacity to consent before I began recruitment and capacity was included within the inclusion criteria given to the gatekeeper. The process of mental capacity assessment was only applicable to my interviews with service-users, despite several staff participants also having a diagnosed mental illness and periods of time under section; this suggests there may be an implicit categorisation amongst service-users and staff.

I also engaged in a discussion with the service-user to determine whether the participant understood the nature of the study, comprehended the study materials, could recognise any personal consequences of participation versus non-participation and had the ability and opportunity to express their choice (Appelbaum \& Grisso, 2001). No concerns relating to participant capacity were raised by the clinical team or myself.

All participants were required to sign a consent form to demonstrate that they understood the research and wished to participate. Participant consent was documented in writing and stored securely in a locked cabinet in accordance with GDPR. Written 
consent was also obtained from Woodlands granting permission for the research and access to the routinely collected data.

I was also mindful of the fact that service-user participants were detained under the MHA and due to this may have felt that engaging in the project was mandatory. To address this, I ensured that all forms of communication emphasised that the research was voluntary.

\subsubsection{Researcher Safety}

As part of my access negotiations and agreements I was required to follow several requirements during data collection. This included receiving a handover prior to interviewing service-users outlining any triggers, warning signs and how the individual preferred to be supported during times of distress, to ensure both service-user and researcher safety. Further requirements relating to my safety included making staff aware of where I was during my time at Woodlands, following their visitor protocol, which included signing in and out of the building and requesting access to certain areas as I was not provided a key card to access all areas, and being aware of their Management of Violence and Aggression policy. This information only referred to my interviews with service-users, which implies a categorisation of service-users and staff, which positions individuals with a mental illness as a risk object.

Aside from physical safety, I also kept a research log and had support from my supervisory team to ensure that any exposure to sensitive content was documented and used for reflexivity purposes to ensure it did not impact upon data collection and analysis. The research log was password protected in accordance with GDPR.

\subsection{Amendments to the Methodology}

Due to the realities of conducting research within a real-world setting, amendments to the project's design were required to ensure the objectives and research question were addressed. Firstly, the two interventions that were going to be the focus of this evaluation were never delivered at Woodlands (see section 8.5.1Deviation from the Intended Research Project). Due to this I decided to focus on recovery because that was a stated output the service was claiming to deliver, and I decided to investigate how this was being achieved. 
With the thesis shifting to focus upon Woodlands as the intervention, the number of referrals the service was receiving was identified as a key barrier to the success of the service. Due to this unexpected difficulty, I wanted to understand this more by introducing commissioner perspectives as they are central to the referrals processes within Wales. By adding a commissioner participant group, I believed that I would be able to begin unpacking explanatory reasons as to why Woodlands was receiving a small number of referrals. Secondly, no service-users nominated a carer for inclusion within this project, therefore I wanted to ensure I used the time I had allocated to these interviews to collect another form of data. This meant that I had a rationale and the time to be able to interview commissioners within this project.

\subsubsection{Commissioners}

I decided to seek views from those responsible for referring to Woodlands to understand the exosystems relevant to Woodlands. A contextual factor that arose out of PA and staff interviews was the wider mental health context and commissioning process in Wales. In order to present a balanced account of this process and to fully understand this exosystem I conducted research interviews with health commissioners who make decisions about appropriate placement of individuals in need of mental health care. Additional recruitment was considered necessary for $\mathrm{CMOC}$ development and to capture exosystem processes that influenced how Woodlands worked at the micro-level. This aligns with the RAMESES standards for RE, which suggests further recruitment should be undertaken if more data is needed to develop or refine CMOCs (Greenhalgh et al., 2017).

An amendment was submitted to REC 6, Health Research Authority (HRA) and Health and Care Research Wales (HCRW) and approval for this additional recruitment was granted on $19^{\text {th }}$ August 2019 (see Appendix Y).

\subsubsection{Recruitment}

Three individuals were identified by the National Collaborative Commissioning Unit who had a list of staff who had referred service-users to Woodlands. Once ethical approval was granted, I contacted the potential participants via their staff email. I provided them with information regarding the project and a PIS (see Appendix Z). Potential participants were 
given the opportunity to ask questions, consent or decline to participate. If participants consented, I sent them a list of dates and times I was available over the next 4-week period and asked them to identify a date and time that was convenient for them. Once a date was arranged, I emailed the participant a consent form to complete prior to the interview (see Appendix AA). I stored this on a password-protected university computer, in accordance with GDPR. A debrief sheet was emailed upon completion of the telephone interview (see Appendix BB).

\subsubsection{Data Collection}

Commissioner interviews took place between August 2019 - October 2019 (see Appendix CC for topic guide). Telephone interviews were considered advantageous for these additional interviews for two reasons. Firstly, telephone interviews could be conducted in a more time efficient manner as it did not mean travelling (Taylor, 2002). Secondly, I could reach geographically dispersed participants as the three participants worked within different health boards across Wales (Aday \& Cornelius, 1996).

I was aware that telephone interviews have been regarded, by some, as inferior to faceto-face interviews (Gillham, 2005), due to a loss of key non-verbal visual data (Novick, 2008), which adds richness to the data (Opdenakker, 2006). However, as non-verbal data was not being considered within my data analysis, I decided that the practical benefits of a telephone interview outweighed the main critique of telephone interviews (Hermanowicz, 2002).

\subsubsection{Analysis}

The framework developed to analyse PA, staff and service-user data could not be applied to commissioner data as these research interviews had a different objective. Commissioner interviews were designed to understand the context of low occupancy at Woodlands as well as the referrals process. The focus was not upon the development and refinement of CMOC's in relation to specific resources available at Woodlands; but instead was considering how Woodlands, as a resource in its own right, was working in the context of the wider mental health system. 
Interview transcripts were analysed thematically (Braun \& Clarke, 2006). The main purpose was to identify and describe patterns within the commissioner data (Braun \& Clarke, 2006). Thematic analysis is not associated with a philosophical stance (Braun \& Clarke 2006), and this flexibility lent itself to the iterative nature of realist research, allowing me to unpack how the context of the referrals system prevented Woodlands from establishing itself as a new service provider. This was important to understand as the low service-user numbers influenced the intended service delivery of Woodlands at the micro-level. The referrals issue was regularly referred to by all participant groups therefore developing further understanding from commissioners' perspective helped to understand the circumstances in which Woodlands, a new, recovery-oriented inpatient service, was not working as intended.

Braun and Clarke (2006) advocate that prior to research commencing, certain decisions relating to the analysis need to be made. My decisions were as follows:

- Firstly, the analysis would be inductive, as opposed to deductive, namely due to the specific focus of the interview to address the referrals process and the low occupancy at Woodlands.

- Secondly, categories would be identified semantically, from the 'explicit or surface meanings of the data' (Braun \& Clarke, 2006, p 84); as opposed to latently, to allow for explicit connection with the data rather than my interpretation. Adopting a sematic approach meant I could simply report commissioners accounts which would be more beneficial in understanding the referrals process and context of low occupancy at Woodlands, without adding my interpretation of what this meant.

- Thirdly, a category was anything that represented something that related to the referrals process or contributed to the low occupancy at Woodlands.

The following phases were then followed when analysing commissioner data (see Table $3)$. 


\begin{tabular}{|c|c|c|}
\hline & Phase & The Process \\
\hline & $\begin{array}{l}\text { Familiarisation } \\
\text { with the data }\end{array}$ & $\begin{array}{l}\text { To immerse myself within the data, all interviews were } \\
\text { transcribed, repeatedly reading, initial ideas were } \\
\text { searched for, and initial meanings and patterns were } \\
\text { identified. }\end{array}$ \\
\hline 2. & $\begin{array}{l}\text { Generating } \\
\text { initial codes }\end{array}$ & $\begin{array}{l}\text { Organising the data in a meaningful and systematic way } \\
\text { reducing the data into small chunks of meaning. This was } \\
\text { done using inductive analysis. }\end{array}$ \\
\hline 3. & $\begin{array}{l}\text { Search for } \\
\text { themes }\end{array}$ & $\begin{array}{l}\text { Once all the data was coded, a long list of different codes } \\
\text { was produced from across the dataset. I began to look at } \\
\text { these codes at a broader level and began sorting and } \\
\text { combining these different codes into potential } \\
\text { overarching categories. }\end{array}$ \\
\hline 4. & $\begin{array}{l}\text { Reviewing } \\
\text { categories }\end{array}$ & $\begin{array}{l}\text { Firstly, the categories were reviewed at the level of the } \\
\text { coded data extracts. This meant ensuring the extracts for } \\
\text { each theme formed a coherent pattern. Secondly, the } \\
\text { validity of the category was considered in relation to the } \\
\text { dataset as a whole. This meant ensuring the categories } \\
\text { reflected the meanings evident in the dataset. }\end{array}$ \\
\hline 5. & $\begin{array}{l}\text { Defining and } \\
\text { naming } \\
\text { categories }\end{array}$ & $\begin{array}{l}\text { Refining the categories to ensure I understood the overall } \\
\text { story of each theme and how these fit together within the } \\
\text { overall story of the data. }\end{array}$ \\
\hline 6. & $\begin{array}{l}\text { Producing the } \\
\text { report }\end{array}$ & $\begin{array}{l}\text { The opportunity to tell the story of the data which } \\
\text { provides a concise, coherent and logical account of the } \\
\text { story the data tells, within and across categories. } \\
\text { Selecting vivid and compelling examples that } \\
\text { demonstrate the merit and validity of the analysis. }\end{array}$ \\
\hline
\end{tabular}

Table 3 - Thematic Analysis from Braun and Clark (2006)

Whilst I acknowledge that other researchers may have identified different categories, I wanted to provide transparency to my data analysis. I have provided the full list of initial codes identified (see Appendix DD), followed by how these were refined into categories, (see Appendix EE). 


\subsection{Reflexivity}

My experiences of conducting the research at the service proved challenging at times. As the service was newly established at the beginning of the $\mathrm{PhD}$, it was continually evolving and developing as the days went by. This impacted the focus and direction of the overall thesis, as the two interventions that were due to be evaluated for this $\mathrm{PhD}$ project were not delivered during the lifetime of the project. As a result of this the project changed considerably a year into my studies as a result of these interventions ceasing to exist at the service, which I regularly noted was a source of frustration and anxiety within my research logs. I was very much aware that this experience with the service could negatively bias me during the data collection and analysis phase. Additionally, there were genuine concerns that the service was going to close due to the uncertainty surrounding referrals; which made me apprehensive as to whether I would have access to any data, the service or potential participants. I found it difficult to separate the uncertainty and frustrations of the limited referrals and potential closure of the service with my analysis of the qualitative data. I felt like my focus became the negative, or pessimistic aspects of service delivery which mirrored some of my encounters with the service, and with some of the participants. My supervisors supported me by providing guidance on evenhandedness and to ensure that I ground my analysis in the data and not in my personal experiences with the service.

\subsection{Summary}

This chapter has situated the research in a philosophical and methodological context that will allow the complexities of Woodlands to be acknowledged throughout the evaluation. This is due to the fact realism embraces confounding variables, rather than attempt to eradicate them in the complex social nature of reality.

In the next chapter I will present a literature review on recovery-oriented inpatient care. 
3 RECOVERY-ORIENTED INPATIENT CARE LITERATURE REVIEW 


\subsection{Introduction}

In this chapter, I critically review the literature on recovery-oriented care (ROC) within inpatient settings. The chapter will present a brief description of mental health recovery, consider how ROC has been defined and provide a rationale for this review. The focus of this review will be to understand how ROC has been implemented within inpatient services. I also consider the context of inpatient settings and whether this facilitates or hinders the successful delivery of ROC. My analysis of the literature will consider how participant groups have different conceptualisations of recovery and ROC within inpatient settings, that there are challenges when delivering ROC within inpatient contexts and I will discuss these challenges in relation to four areas of care.

Recovery is understood in several ways (Silverstein \& Bellack, 2008) and remains a debated and controversial concept. Recovery is referred to as a process of how an individual overcomes the challenges associated with mental illness (Anthony, 1993; Davidson et al., 2005); whereas some mental health professionals orient towards considering recovery in terms of symptomatology and improvements in mental health outcomes (Lieberman et al., 2008). Others have moved away from the clinical or serviceuser definitions of recovery to consider the role of the community in supporting recovery (Onken et al., 2007). This highlights that there is a lack of consensus of what recovery means.

Despite this lack of clarity, the discussion of recovery is becoming ever more prominent in mental health treatment. The notion of mental health recovery acknowledges that the recovery journey is unique and personal to each individual (Anthony, 1993; Deegan, 2002). Originating from service-user perspectives that challenged traditional beliefs about mental health and treatment, recovery has been conceptualised as a process of building a meaningful and satisfying life, even if the individual has ongoing or recurring symptoms or problems associated with a mental illness (Anthony, 1993). It has been suggested that this view of recovery reforms how we understand and manage mental illness, as well as how individuals living with a mental illness are supported and helped (Farkas, 2007). This means mental health services need to reconsider their role in supporting individuals in their recovery. However, Anthony's (1993) construction of recovery is located within a 
highly specific cultural system, the United States of America (USA), which may view individuals as both the problem and the solution and deviate from service-user defined recovery (Deegan, 1988). Despite this critique, there has been an increasing drive for a transformation of the whole mental health system and a shift towards recoveryorientation and the support of personal recovery (Piat et al., 2017).

Whilst it is acknowledged that attempts have been made to scientifically frame serviceuser recovery (Leamy et al., 2011), these have been criticised for focusing upon persons who are deemed to have 'recovered', meaning less is known about the experiences of those who continue to struggle (Rose, 2014). The initial construction of service-user defined recovery stood in opposition to individualism and notions of success and cure (Deegan, 1988). Therefore, framing recovery as a clinical outcome may prevent serviceuser defined recovery from truly entering or guiding our services. It has been argued that if we rely upon the processes and stories of those who professionals deem to be 'recovered', the concept of service-user recovery is rendered meaningless (Onken et al., 2007). This suggests that mental health recovery transcends any model or theory and instead is about the unique, personal process an individual goes through. Deegan (1989) argued against recovery being reduced to a set of systemised principles which suggests that the attempts to operationalise recovery through incorporating ROC within policy may be a deviation from service-user advocates. If recovery is so idiosyncratic then this presents a problem for services when attempting to standardise recovery for service delivery.

ROC has featured within mental health policies across many Western countries, such as the United Kingdom (Department of Health, 2011), Australia (Australian Government, 2009) and Canada (Mental Health Commission of Canada, 2009). Analysis of international practice guidelines identified four key recovery-relevant themes: promoting citizenship, organisational commitment, supporting personally defined recovery and working relationships (Le Boutillier et al., 2011). Le Boutillier et al's (2011) paper highlights that wider system acceptance of ROC is needed for its implementation at a provider-level, alluding to the need for a system-wide shift. However, the system context that ROC, or recovery-oriented services have had to operate within is largely medical dominated (Morera, Pratt, \& Bucci, 2017), paternalistic (Knaak et al., 2017), focused on a cure (Slade 
et al., 2014), and wanting to reduce hospital beds (Ewbank et al., 2017), which conflicts with the notions of recovery (Deegan, 1988); highlighting system wide challenges to delivering ROC.

There is ongoing debate as to whether all mental health services can achieve the expectation of embracing and delivering ROC, specifically services that provide compulsory treatment under mental health legislation (Simpson \& Penney, 2011). The implementation of ROC within these settings has been criticised for providing 'imposed recovery'; where recovery is forced upon individuals, compromising choice and autonomy and stifling hope (Young, 2011, p. 397). This debate could stem from the fact that ROC principles originated from community and outpatient settings (Whitley et al., 2009; Compton et al., 2014). This raises the question of whether these principles are compatible with inpatient care, and whether these principles can simply be transferred into inpatient care. Inpatient care focuses upon crisis-management, uses coercive treatment, and advocates pharmacological approaches, which have the potential to conflict with key recovery processes, such as self-determination, hope and empowerment (Stylianos \& Kehyayan, 2012). It appears that the characteristics of inpatient care may complicate the implementation of ROC and therefore consideration of these characteristics and ways to address or overcome these challenges is needed to fulfil expectations of ROC.

Whilst key components of recovery, such as having a meaningful and purposeful life, are well-documented within literature (Anthony, 1993; Davidson et al., 2009), the how's of ROC and the practical application of these concepts has received less attention; with even less attention given to its implementation and impact within inpatient contexts (Davidson et al., 2016). It is hoped that the consideration of ROC in inpatient settings could help to assist services and mental health professionals in their shift towards ROC. Developing our knowledge of how and why ROC does, or does not work, within inpatient settings, could begin to build an understanding of what is necessary for ROC to successfully work within the context of inpatient settings.

The purpose of this literature review will be to identify and summarise how inpatient services have operationalised ROC. The scope of this review will address these questions: 
1. What are the barriers to delivering ROC within the context of inpatient services?

\section{What resources (mechanism) are used to deliver ROC in inpatient services, and what outcomes are associated with these resources?}

This literature review will inform the subsequent research project as the service under evaluation aims to deliver ROC within the context of an inpatient service. Focusing upon inpatient ROC meant that I could begin to understand the resources other services have provided to support individuals in their recovery and how these have worked in supporting individuals in their recovery. In addition to this, it will enable me to identify context specific factors that may have influenced how these resources worked within inpatient care, which may be relevant to Woodlands. The summary of the current evidence base and what I learn from this review will be used to inform the interviews conducted with programme architects and provide me with background knowledge of how ROC is expected to work within the context of inpatient care, which I will be able to adapt and refine to be specific to Woodlands.

Whilst it is acknowledged that a review of ROC within inpatient settings already exists (Waldemar et al., 2015), the focus of this review was the extent to which ROC approaches were integrated into this setting. Whereas, the current review will focus upon the how's of ROC within an inpatient context, considering what resources are implemented and identify any aspects of the inpatient context which support or hinder the operationalisation of ROC.

\subsection{Search Strategy}

An initial search was carried out during June 2018; a repeat of this search was carried out in April 2020 to incorporate more recent literature that was not available at the time of the original search (see Table 4). Advice on the search strategy was sought from a Subject Librarian for Health and Medicine at Swansea University. The subject Librarian was asked to review the search terms I had selected and to provide feedback as to whether this was an appropriate search for the scope of this review. 


\begin{tabular}{c|c|c}
$\begin{array}{c}\text { Recovery-Oriented Care - } \\
\text { Title }\end{array}$ & Setting - Abstract & Population - Abstract \\
\hline recover* OR recovery* OR & inpatient* OR in- & mental illness* OR mental \\
recovery-orient* OR & patient* OR & disorder* OR mental disease* OR \\
recovery orient* OR & resident* OR & mental problem* OR psychiatric \\
recovery-focus* OR & hospital* OR unit* & health OR psychiatric illness OR \\
recovery focus* OR & OR recovery centre & psychiatric disorder OR psychiatric \\
recovery based practice OR & OR rehab* & problem OR mental well* OR \\
personal recovery & & mental health*
\end{tabular}

Table 4 - Search Terms Used

I decided to not use synonyms for the word 'recovery' because I wanted to focus specifically upon how services work in ways which promote and support personal recovery and the service-user narrative of recovery. Due to this, words such as rehabilitation were excluded as this term is often adopted by professionals and services, rather than service-users (Slade et al., 2014); which would be a deviation from the intention of this review which was looking at how service-user defined recovery was being fulfilled/achieved by services. I was aware that some have suggested that personal recovery, which relates individuals with complex and serious mental illness (Recovery in the Bin, 2019), could be a beneficial template for rehabilitation services which support this demographic of service-user (Holloway, 2010), therefore I included 'rehab*' within the abstract search relating to the setting as this could be an inpatient context, like Woodlands, that aims to provide ROC.

I also decided to look at the title of papers that were used within a 2015 review of recovery-oriented inpatient care (Waldemar et al., 2015) to see what terms featured in the titles used in this review. I noted that mental health-related terms and inpatient related terms were not always within the title and therefore decided to extend my search by looking for these terms within the abstract. Although I knew this would generate a large search result and may include physical health conditions and settings, I considered 
this the most appropriate means of identifying all papers on ROC in mental health inpatient settings. I also noted that recovery related terms featured in all the titles, and as this was the main area of interest, I decided to restrict these search terms to the title only. The subject Librarian cautioned that the results from the search were high and advised I applied extra restrictions, such as from a certain date, or limiting the search terms to the title. However, after removing the duplicates and quickly scanning the titles of the first 200 papers and noting a number relating to physical health conditions, I considered this to be a manageable size of papers for me to review (see Figure 6).

The following databases were searched:

- Applied Social Sciences Index \& Abstracts (ASSIA)

- British Nursing Database

- The Cumulative Index to Nursing and Allied Health Literature Database (CINAHL)

- MEDLINE

- ProQuest Central

- PsycINFO

- PsycARTICles

- Web of Science

My rationale for selecting these databases was supported by the topic area of mental health. When choosing the databases, care was taken to utilise resources from medical, psychological and social perspectives to increase the chances of retrieving relevant papers. My decision to look at these specific areas was supported by the widely accepted biopsychosocial model for engaging with mental distress (Engel, 1977). The model provides a holistic approach to the development of mental ill-health by explaining the complex interplay of three major dimensions (biological, psychological and social). This model therefore informed the selection of the databases used within this review.

\subsubsection{Inclusion Criteria}

The following criteria was essential for inclusion within the review: 
- The paper related to ROC within an inpatient setting - there were no parameters on what the inpatient setting was, so this included acute, short-stay and long-term care;

- The paper was an empirical paper which involved collecting or analysing original data;

- The paper included adult participants;

- The paper was written in English.

In addition to the above criteria the paper had to meet at least one of the following criteria:

- The paper discussed how the inpatient service claimed to deliver ROC;

- The paper evaluated the outcomes of ROC;

- The paper discussed barriers and facilitators to ROC within the context of inpatient settings.

\subsubsection{Exclusion Criteria}

The formation of the exclusion criteria was on an iterative process and was developed from the emerging data (see Figure 6). The following exclusion criteria was developed:

- The paper referred to non-adult participants;

- The paper was a literature review of personal recovery, ROC or ROC policy;

- The paper referred to settings that were not inpatient facilities;

- The paper related to the development of a measure to assess the recoveryorientation of a service;

- The paper related to a physical health condition or setting;

- The paper related to defining personal recovery and not about its application by services within practice;

- The paper related to the evaluation of staff training relating to ROC;

- The paper evaluated a specific ROC programme or intervention, such as a recovery group or wellness recovery action planning;

- The paper was not in English. 
It is important to note here that I am aware a realist review of the literature would include grey literature (such as government policies, brochures, editorials). The reason grey literature was excluded from this review was due to time - I spent the first year completing two separate realist reviews on Acceptance and Commitment Group for Psychosis and Physical Health Education for those with serious mental illness, however these interventions were never delivered at the service. Due to this my literature reviews and entire thesis direction changed, and I did not have the time to complete a comprehensive review of all the literature, therefore I focused solely upon empirical papers.

\subsubsection{Relevance}

This involved determining if the content was appropriate for the purpose and scope of the review. This meant making a judgement about whether it contributed to what ROC meant in inpatient contexts, how ROC was achieved within inpatient settings, barriers to ROC in inpatient settings and any outcomes associated with ROC. The relevance of academic literature was initially determined from a title sift, then an abstract sift, and then a full text sift. If the abstract was ambiguous but suggested it might be relevant, the full document was read (see Figure 6).

I deliberately excluded papers that related to a specific recovery group or intervention as I wanted to see how ROC guided the overall delivery of inpatient services. For example, the following papers were excluded on this basis they were a specific intervention aimed at supporting recovery - a group-based lifestyle intervention for adults with serious mental illness (Aschbrenner et al., 2013), a self-help group for people recovering from mental illness (Leung \& Arthur, 2004) and a recovery group for people with severe and enduring mental health problems (Gillespie \& Clarke, 2007). The reason for this was because the service under evaluation, Woodlands, was aiming to provide a recoveryoriented inpatient service and therefore I wanted to identify literature that had researched how this had been achieved elsewhere, as opposed to a specific one-off intervention aimed at promoting recovery. I also deliberately excluded papers that related to staff training in recovery, or ROC because I wanted to focus upon the resources provided specifically to service-users to support their recovery. Whilst I note that the 
development of staff skills and knowledge of recovery is likely to have a ripple effect on service-user recovery, I wanted to look at the direct and practical things services were using as a means of promoting ROC.

I was also aware that there is a large body of literature that considers the concept of recovery and processes of recovery, and therefore did not consider a review of this literature necessary for the purposes of this review as I was looking at recovery specifically within the context of inpatient settings, and wanting to understand how services were facilitating ROC. Due to these decisions, very specific papers were being sought, which considered how ROC was applied into inpatient settings as a whole, rather than individual interventions that were aimed at addressing key recovery approaches, such as hope.

I was therefore aiming to identify papers that specifically focused upon how ROC was delivered as a philosophy of care in inpatient settings, what were the associated outcomes of the resources used to deliver ROC and whether the context of inpatient care influenced services ability to deliver ROC.

\subsubsection{Quality Appraisal}

Upon identifying relevant research using the inclusion criteria I appraised the quality of this research by using a range of quality appraisal tools. As I was not focused on a specific method or research design I used the Critical Appraisal Skills Programme (CASP) (Critical Appraisal Skills Programme, 2014) for qualitative research, the Mixed Methods Appraisal Tool (MMAT) (National Collaborating Centre for Methods and Tools, 2011) for mixed methods research and the HCPRDU Evaluation Tool for quantitative studies (Long \& Godfrey, 2004). These tools are widely used in health research, are user-friendly, valid, accessible and appropriate to the topic of this review.

- CASP for qualitative research was designed to address validity, results and the relevance to practice. The qualitative tool comprised eleven different questions and assessed criteria related to the validity of the research, such as: was there a clear statement of the aims of the research? Is a qualitative methodology appropriate? Was the recruitment strategy appropriate to the aims of the research? but also evaluating the results (Have ethical issues been taken into consideration? Is there a clear statement of findings?) and will the results help 
locally (How valuable is the research?). I rated these quality appraisal questions either Yes, No or Can't Tell, and considered these to determine the quality of the research available (see Appendix FF)

- The HCPRDU Evaluation Tool for quantitative studies (Long \& Godfrey, 2004), contains 51 questions across six subsections: study evaluative overview; study, setting and sample; ethics; group comparability and outcome measurement; policy and practice implications; and other comments. Due to the number of questions in this tool, I have not included my responses to each of these questions, however each quantitative study was considered to have enough quality to be included within this review (see Appendix GG).

- The MMAT for mixed methods research comprises of 5 questions: the rationale for mixed methods, the different components of the study, the outputs of the integration, are divergences and inconsistencies addressed and does the study adhere to the quality criteria of each tradition of the methods involved. Responses to these questions are yes, no or can't tell (see Appendix $\mathrm{HH}$ ).

Each paper included within this review was assessed for quality (see Table 5). No papers were excluded based upon their quality. 


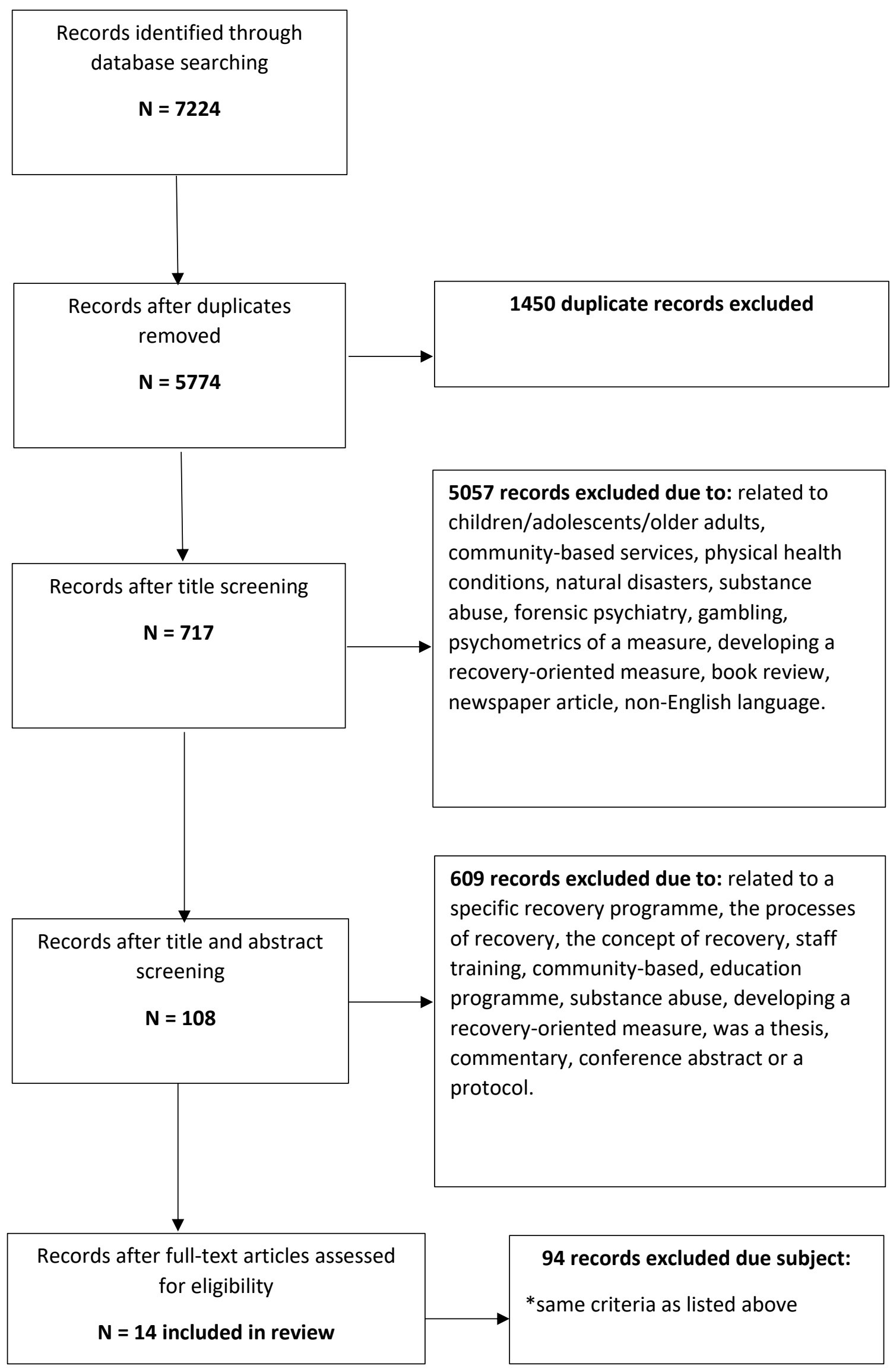

Figure 6 - Flowchart of Screening Process 


\subsubsection{Papers Included}

14 papers met the inclusion criteria and were included within this review (see Table 5). Selected papers related to how ROC was delivered in inpatient settings, barriers of ROC within the context of inpatient services, or outcomes associated with ROC. All papers were quality appraised using one of three tools. In Table $2 \mathrm{I}$ indicate which tool has been used for which paper and include my responses to the specific questions within each tool. Although all the studies were appraised for quality, I will now discuss the design of these papers, as well as any notable limitations. Despite all the papers being appraised for quality, the papers used ranged in research designs, data collection methods, size of the study (number of sites and participants), meaning some papers were considered more robust or transferable than others.

One study used mixed methods, which included survey data from 301 service-users and 290 staff, in addition to 76 interviews with service-users, staff and carers across 19 wards by 6 different providers across England and Wales (Coffey et al., 2019a). The number of sites and participants included within this study increased the transferability of these findings as it was not limited to one service. This was considered as one of the more robust papers in this review. However, it was not without its limitations, the number of staff and carers involved in the study fell short of the recruitment numbers which were identified based upon power and the study reported that there was missing data. The integration of the two methods in the analysis could also have been improved to identify further areas of converge, divergence and complimentary findings given the volume of data available. Despite these limitations, this was considered a reliable paper within this review due to capturing data from across several mental health services in the UK. Also, as this paper was conducted within the UK, it sits within the same socio-political landscape as the service under evaluation and may be more transferable than other papers from different cultures and countries.

One study used focused ethnographic method, combining 84 hours of participant observation and informal interviews (Waldemar et al., 2018a), meaning the findings were not just dependent upon what participants said about ROC at the service, but included observations and field notes from an independent researcher. The researcher did, 
however, miss some encounters such as sporadic one-to-ones and the role and interactions of peer mentors (PMs). Generalisability is not the aim of ethnographic enquiry (Hammersley \& Atkinson, 2007), instead it allowed for the complex nature of the individual service to be characterised, which was beneficial for the scope of this review. Although the validity and reliability of ethnographic research has long been debated (Compte \& Goetz, 1982), this was considered a valuable paper to address the context and complexity of ROC in inpatient care.

Two papers used qualitative methods combined with a review of the literature (Chen et al., 2013; Gwinner \& Ward, 2015), which added to the robustness of the research as the authors converged the data from the different resources. Gwinner and Ward (2015) focused on one site and conducted a focus group initially with 12 nurses, and then a further 45 nurses. Chen et al (2013) interviewed a range of participants (3 service-users, 3 family members, 2 community nurses with previous experience of inpatient services, 5 staff members from various disciplines working in inpatient care, 2 educators in strategic positions) from a range of inpatient settings which increased the transferability of these findings compared to Gwinner and Ward's (2015) paper.

Four papers reported the use of research interviews; Hungerford and Richardson (2013) conducted semi-structured interviews with 10 carers, Cleary et al (2013) conducted semistructured interviews with 21 nurses, Waldemar et al., (2018b) conducted semistructured interviews with 14 service-users, and De Ruysscher et al (2020) conducted semi-structured interviews with 10 service-users and 10 staff. Across the four papers, which all evaluated different inpatient services, all staff participants were still working at the service at the time of being interviewed, and all service-users were still accessing the service at the time of the interviews.

Hungerford and Fox (2014) conducted a combination of research interviews and focus groups with 9 service-users ( 3 had individual interviews and 6 were in a focus group). The authors reported using interpretative phenomenological analysis, however, there was a reliance upon what participants explicitly said meaning there was no focus on how participants understood what they were describing. This meant that factors of importance in IPA, such as linguistic comments around the use of language were not considered and 
the context of each participant, were not considered. This meant that there was an absence of how the theoretical underpinnings of IPA were applied within this study.

Three papers used focus groups, McKenna et al (2013) recruited 46 nurses from 5 different inpatient services which added to the transferability and reliability of the findings due to the use of multiple sites. However, the focus group questions were leading rather than open-ended which could influence the validity of the findings. Aston and Coffey (2012) conducted focus groups with 6 service-users who had previous experience of inpatient care, and 5 nurses who were currently working in inpatient care. This paper had a small sample size and was retrospective for service-users as they were no longer accessing inpatient care, therefore important aspects of ROC may have been lost. This paper did, however, add value to the conceptualisation of recovery and ROC and usefully shows different understandings of recovery between groups and that when we talk about recovery we are not always talking about the same construct.

Chisholm et al (2020) conducted focus groups with 8 PMs, which represented only $25 \%$ of the PMs working at the service, and 7 out of the 8 participants were female which may bias the findings. The focus groups in Chisholm et al (2020) were conducted by an individual who also worked at the service, therefore, the position of this individual within the organisation may have influenced participant responses. This raises questions over the reliability of these findings; however, an advantage of this specific paper was it was the only paper to include PMs as a stakeholder group, which is in keeping with ROC. The paper used a social constructivist approach, however made little reference to how this approach theoretically underpinned their data collection, analysis and findings. The authors did refer to a process where the researcher scribed and made notes about themes and checked back with participants for clarification, as well as detailing the relationship of the researcher to the service. However, the authors did not consider how the interactions between the researcher and the participants would have influenced the construction of knowledge, or how this would have limited what conclusions could be drawn.

Two papers examined survey data which included data from 910 staff (Tsai \& Salyers, 2010) and 60 service-users (Osbourn \& Stein, 2019). Causality was unknown meaning the findings could not be attributed to staff characteristics or ROC. The validity of some of the 
measures used within these papers can also be questioned as they were not designed specifically for use within inpatient contexts, nor had they been validated for inpatient populations. These papers related to staff characteristics within inpatient settings and how these could influence the delivery of ROC, which was valuable considering the role and resource of staff was frequently identified as essential to ROC within the qualitative papers.

\begin{tabular}{|c|c|c|}
\hline Paper & Design & Quality Appraisal \\
\hline $\begin{array}{l}\text { Aston, V., \& Coffey, M. } \\
\text { (2012). Recovery: what } \\
\text { mental health nurses and } \\
\text { service users say about the } \\
\text { concept of } \\
\text { recovery. Journal of } \\
\text { Psychiatric and Mental } \\
\text { Health Nursing, 19(3), 257- } \\
263 .\end{array}$ & $\begin{array}{l}\text { Setting - Inpatient mental health settings - } \\
\text { did not specify if acute, but acute settings } \\
\text { were referred to by participants. } \\
\text { Data Collection - Focus groups with adult } \\
\text { service-users group ( } n=6) \text { who had } \\
\text { previous experience or recent experience } \\
\text { of inpatient mental health services. Focus } \\
\text { group with nursing group ( } n=5 \text { ) currently } \\
\text { working in inpatient care. } \\
\text { Data Analysis - Framework analysis. } \\
\text { Country - UK. }\end{array}$ & $\begin{array}{l}\text { - Used CASP } \\
\text { for } \\
\text { qualitative } \\
\text { research } \\
\text { 1. Yes } \\
\text { 2. Yes } \\
\text { 3. Yes } \\
\text { 4. Can't tell } \\
\text { 5. Yes } \\
\text { 6. No } \\
\text { 7. Yes } \\
\text { 8. Yes } \\
\text { 9. Yes } \\
\text { 10. Yes }\end{array}$ \\
\hline $\begin{array}{l}\text { Chen, S. P., Krupa, T., } \\
\text { Lysaght, R., McCay, E., \& } \\
\text { Piat, M. (2013). The } \\
\text { development of recovery } \\
\text { competencies for in- } \\
\text { patient mental health } \\
\text { providers working with } \\
\text { people with serious mental } \\
\text { illness. Administration and } \\
\text { Policy in Mental Health } \\
\text { and Mental Health Services } \\
\text { Research, 40(2), 96-116. }\end{array}$ & $\begin{array}{l}\text { Setting - acute and long-term inpatient } \\
\text { mental health settings. } \\
\text { Data Collection - Semi-structured } \\
\text { interviews with multiple stakeholders } \\
\text { (service-users, staff, significant others, } \\
\text { managers) and literature review. } \\
\text { Data Analysis - Literature review and } \\
\text { thematic analysis to develop a } \\
\text { competency framework. } \\
\text { Location - Canada }\end{array}$ & $\begin{array}{l}\text { - Used CASP } \\
\text { for } \\
\text { qualitative } \\
\text { research } \\
\text { 1. Yes } \\
\text { 2. Yes } \\
\text { 3. Yes } \\
\text { 4. Yes } \\
\text { 5. Yes } \\
\text { 6. Can't tell } \\
\text { 7. Yes } \\
\text { 8. Can't tell } \\
\text { 9. Yes } \\
\text { 10. Yes }\end{array}$ \\
\hline $\begin{array}{l}\text { Chisholm, J., \& Petrakis, M. } \\
\text { (2020). Peer Worker } \\
\text { Perspectives on Their } \\
\text { Potential Role in the } \\
\text { Success of Implementing } \\
\text { Recovery-Oriented } \\
\text { Practice in a Clinical } \\
\text { Mental Health }\end{array}$ & $\begin{array}{l}\text { Setting - inpatient setting. } \\
\text { Data Collection - Focus group with } 8 \text { peer } \\
\text { mentors - } 7 \text { female, } 1 \text { male. } \\
\text { Data Analysis - Thematic analysis. } \\
\text { Location - Australia. }\end{array}$ & $\begin{array}{l}\text { - } \begin{array}{l}\text { Used CASP } \\
\text { for } \\
\text { qualitative } \\
\text { research }\end{array} \\
\text { 1. Yes } \\
\text { 2. Yes } \\
\text { 3. Yes }\end{array}$ \\
\hline
\end{tabular}




\begin{tabular}{|c|c|c|}
\hline $\begin{array}{l}\text { Setting. Journal of } \\
\text { Evidence-Based Social } \\
\text { Work, 1-17. }\end{array}$ & & $\begin{array}{l}\text { 4. No } \\
\text { 5. Yes } \\
\text { 6. Yes } \\
\text { 7. Yes } \\
\text { 8. Yes } \\
\text { 9. Yes } \\
\text { 10. Yes }\end{array}$ \\
\hline $\begin{array}{l}\text { Cleary, M., Horsfall, J., } \\
\text { O'Hara-Aarons, M., \& } \\
\text { Hunt, G. E. (2013). Mental } \\
\text { health nurses' views of } \\
\text { recovery within an acute } \\
\text { setting. International } \\
\text { Journal of Mental Health } \\
\text { Nursing, 22(3), 205-212. }\end{array}$ & $\begin{array}{l}\text { Setting - acute inpatient mental health. } \\
\text { Data Collection - Interviewed } 21 \text { nurses. } \\
\text { Data Analysis - Thematic analysis. } \\
\text { Location - Australia. }\end{array}$ & $\begin{array}{l}\text { - Used CASP } \\
\text { for } \\
\text { qualitative } \\
\text { research } \\
\text { 1. Yes } \\
\text { 2. Yes } \\
\text { 3. Yes } \\
\text { 4. Yes } \\
\text { 5. Yes } \\
\text { 6. Can't tell } \\
\text { 7. Yes } \\
\text { 8. No } \\
\text { 9. Yes } \\
\text { 10. Yes }\end{array}$ \\
\hline $\begin{array}{l}\text { Coffey, M., Hannigan, B., } \\
\text { Barlow, S., Cartwright, M., } \\
\text { Cohen, R., Faulkner, A., \& } \\
\text { Simpson, A. (2019a). } \\
\text { Recovery-focused mental } \\
\text { health care planning and } \\
\text { co-ordination in acute } \\
\text { inpatient mental health } \\
\text { settings: a cross national } \\
\text { comparative mixed } \\
\text { methods study. BMC } \\
\text { psychiatry, 19(1), } 115 .\end{array}$ & $\begin{array}{l}\text { Setting - nineteen inpatient mental health } \\
\text { wards, across six providers - } 1 \text { admissions, } \\
2 \text { acute and } 3 \text { inpatient wards. } \\
\text { Data Collection - A survey of service-users } \\
\text { ( } n=301 \text { ) and staff ( } n=290 \text { ) and embedded } \\
\text { case studies involving interviews with } \\
\text { staff, service-users and carers ( } n=76) \text {. } \\
\text { Data Analysis - Framework analysis and } \\
\text { ANOVA and ANCOVA analysis. } \\
\text { Location - England and Wales. }\end{array}$ & 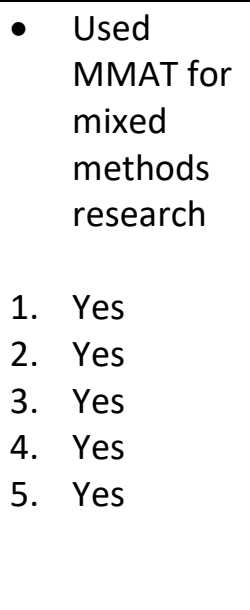 \\
\hline $\begin{array}{l}\text { De Ruysscher, C., } \\
\text { Vandevelde, S., Tomlinson, } \\
\text { P., \& Vanheule, S. (2020). } \\
\text { A qualitative exploration of } \\
\text { service users' and staff } \\
\text { members' perspectives on } \\
\text { the roles of inpatient } \\
\text { settings in mental health } \\
\text { recovery. International } \\
\text { Journal of Mental Health } \\
\text { Systems, 14(1). }\end{array}$ & $\begin{array}{l}\text { Setting - residential unit, housing project, } \\
\text { case management - focus on inpatient } \\
\text { part of service. } \\
\text { Data Collection - Interviews with service- } \\
\text { users and staff members about the daily } \\
\text { practice of a recovery-oriented ward. } 7 \\
\text { men, } 3 \text { women service-users. Staff } \\
\text { interviews - psychiatrist, } 2 \text { psychologists, } \\
\text { social workers, } 2 \text { nurses, a pedagogical } \\
\text { staff member, } 3 \text { occupational therapists. } \\
\text { Data Analysis - Thematic analysis. } \\
\text { Location - Belgium. }\end{array}$ & $\begin{array}{l}\text { - Used CASP } \\
\text { for } \\
\text { qualitative } \\
\text { research } \\
\text { 1. Yes } \\
\text { 2. Yes } \\
\text { 3. Yes } \\
\text { 4. Can't tell } \\
\text { 5. Yes } \\
\text { 6. No } \\
\text { 7. Yes } \\
\text { 8. No } \\
\text { 9. Yes } \\
\text { 10. Yes. }\end{array}$ \\
\hline
\end{tabular}




\begin{tabular}{|c|c|c|}
\hline $\begin{array}{l}\text { Gwinner, K., \& Ward, L. } \\
\text { (2015). Storytelling, } \\
\text { Safeguarding, Treatment, } \\
\text { and Responsibility: } \\
\text { attributes of recovery in } \\
\text { psychiatric intensive care } \\
\text { units. Journal of Psychiatric } \\
\text { Intensive Care, 11(2), 105- } \\
118 .\end{array}$ & $\begin{array}{l}\text { Setting - Locked Psychiatric Intensive Care } \\
\text { Unit. } \\
\text { Data Collection - Focus groups, literature } \\
\text { review followed by another focus group. } \\
12 \text { nurses participated in the first two } \\
\text { focus groups, an additional focus group } \\
\text { was held to accommodate desire ( } \mathrm{n}=45 \text { ) } \\
\text { Data Analysis - Thematic analysis. } \\
\text { Location - Australia. }\end{array}$ & $\begin{array}{ll}- & \begin{array}{l}\text { Used CASP } \\
\text { for }\end{array} \\
& \text { qualitative } \\
& \text { research } \\
\text { 1. } & \text { Yes } \\
\text { 2. } & \text { Yes } \\
\text { 3. Yes } \\
\text { 4. Yes } \\
\text { 5. Yes } \\
\text { 6. Can't tell } \\
\text { 7. Yes } \\
\text { 8. Yes } \\
\text { 9. Yes } \\
\text { 10. } \text { Yes }\end{array}$ \\
\hline $\begin{array}{l}\text { Hungerford, C., \& Fox, C. } \\
\text { (2014). Consumer's } \\
\text { perceptions of Recovery- } \\
\text { oriented mental health } \\
\text { services: An Australian } \\
\text { case-study } \\
\text { analysis. Nursing \& health } \\
\text { sciences, 16(2), 209-215. }\end{array}$ & $\begin{array}{l}\text { Setting - Public mental health service. } \\
\text { Data Collection - Semi-structured } \\
\text { interview schedule with } 9 \text { consumers. ( } 3 \\
\text { asked to be interviewed individually, } 6 \text { as } \\
\text { part of a focus group). } \\
\text { Data Analysis - IPA } \\
\text { Location - Australia. }\end{array}$ & $\begin{array}{ll}\text { - } & \text { Used CASP } \\
\text { for } \\
\text { qualitative } \\
\text { research }\end{array}$ \\
\hline $\begin{array}{l}\text { Hungerford, C., \& } \\
\text { Richardson, F. (2013). } \\
\text { Operationalising Recovery- } \\
\text { oriented services: The } \\
\text { challenges for } \\
\text { carers. Advances in Mental } \\
\text { Health, 12(1), 11-21. }\end{array}$ & $\begin{array}{l}\text { Setting - Public mental health service. } \\
\text { Data Collection - semi-structured } \\
\text { interviews with } 10 \text { people who self- } \\
\text { identified as a carer of a person with a } \\
\text { chronic or severe mental illness who was } \\
\text { accessing the mental health service. } \\
\text { Data Analysis - IPA } \\
\text { Location - Australia. }\end{array}$ & $\begin{array}{ll}\text { - } & \text { Used CASP } \\
\text { for } \\
\text { qualitative } \\
\text { research }\end{array}$ \\
\hline $\begin{array}{l}\text { McKenna, B., Furness, T., } \\
\text { Dhital, D., Ennis, G., } \\
\text { Houghton, J., Lupson, C., \& } \\
\text { Toomey, N. (2014). } \\
\text { Recovery-oriented care in } \\
\text { acute inpatient mental }\end{array}$ & $\begin{array}{l}\text { Setting - } 5 \text { acute mental health services. } \\
\text { Data Collection - Focus group interviews } \\
\text { with mental health nurses. } 46 \text { nurses with } \\
\text { a range of experiences and from a range } \\
\text { of countries. }\end{array}$ & $\begin{array}{l}\text { - Used CASP } \\
\text { for } \\
\text { qualitative } \\
\text { research } \\
\text { 1. Yes }\end{array}$ \\
\hline
\end{tabular}




\begin{tabular}{|c|c|c|}
\hline $\begin{array}{l}\text { health settings: An } \\
\text { exploratory study. Issues in } \\
\text { Mental Health } \\
\text { Nursing, 35(7), 526-532. }\end{array}$ & $\begin{array}{l}\text { Data Analysis - General inductive } \\
\text { approach. } \\
\text { Location - Australia. }\end{array}$ & $\begin{array}{ll}\text { 2. Yes } \\
\text { 3. Can't tell } \\
\text { 4. Yes } \\
\text { 5. Yes } \\
\text { 6. Yes } \\
\text { 7. Yes } \\
\text { 8. Can't tell } \\
\text { 9. Yes } \\
\text { 10. Yes } \\
\end{array}$ \\
\hline $\begin{array}{l}\text { Osborn, L. A., \& Stein, C. H. } \\
\text { (2019). Recovery-oriented } \\
\text { services in an inpatient } \\
\text { setting: The role of } \\
\text { consumers' views of } \\
\text { therapeutic alliance and } \\
\text { practitioner directiveness } \\
\text { on recovery and well- } \\
\text { being. American Journal of } \\
\text { Orthopsychiatry, 89(1), } \\
\text { 115. }\end{array}$ & $\begin{array}{l}\text { Setting - Inpatient psychiatric hospital } \\
\text { Data Collection - } 60 \text { clients ( } 47 \text { men; } 13 \\
\text { women), completed survey data. } \\
\text { Data Analysis - T-tests and one-way } \\
\text { anova. } \\
\text { Location - USA. }\end{array}$ & $\begin{array}{l}\text { Used the HCPRDU } \\
\text { Evaluation Tool for } \\
\text { quantitative } \\
\text { studies (Long \& } \\
\text { Godfrey, } 2004 \text { and } \\
\text { completed the } 51 \\
\text { questions. }\end{array}$ \\
\hline $\begin{array}{l}\text { Tsai, J., \& Salyers, M. P. } \\
\text { (2010). Recovery } \\
\text { orientation in hospital and } \\
\text { community settings. The } \\
\text { journal of behavioural } \\
\text { health services \& } \\
\text { research, 37(3), 385-399. }\end{array}$ & $\begin{array}{l}\text { Setting - Inpatient staff compared to } \\
\text { community staff. } \\
\text { Data Collection - Survey data - } 729 \\
(63.4 \%) \text { staff members from three state } \\
\text { hospitals and } 181(78.7 \%) \text { from } \\
\text { community from a range of disciplines. } \\
\text { Data Analysis - t-tests and ancova. } \\
\text { Location - USA. }\end{array}$ & $\begin{array}{l}\text { Used the HCPRDU } \\
\text { Evaluation Tool for } \\
\text { quantitative } \\
\text { studies (Long \& } \\
\text { Godfrey, } 2004 \text { and } \\
\text { completed the } 51 \\
\text { questions. }\end{array}$ \\
\hline $\begin{array}{l}\text { Waldemar, A. K., } \\
\text { Esbensen, B. A., Korsbek, } \\
\text { L., Petersen, L., \& Arnfred, } \\
\text { S. (2018a). Recovery- } \\
\text { oriented practice: } \\
\text { Participant observations of } \\
\text { the interactions between } \\
\text { patients and health } \\
\text { professionals in mental } \\
\text { health inpatient } \\
\text { settings. International } \\
\text { journal of mental health } \\
\text { nursing, 28(1), 318-329. }\end{array}$ & $\begin{array}{l}\text { Setting - Open inpatient ward and locked } \\
\text { intensive care ward. } \\
\text { Data Collection - Focused ethnographic } \\
\text { method - using participant observation } \\
\text { and informal interviews to study service- } \\
\text { user and staff interactions. } \\
\text { Data analysis - Field notes analysed using } \\
\text { qualitative content analysis. } \\
\text { Location - Denmark }\end{array}$ & 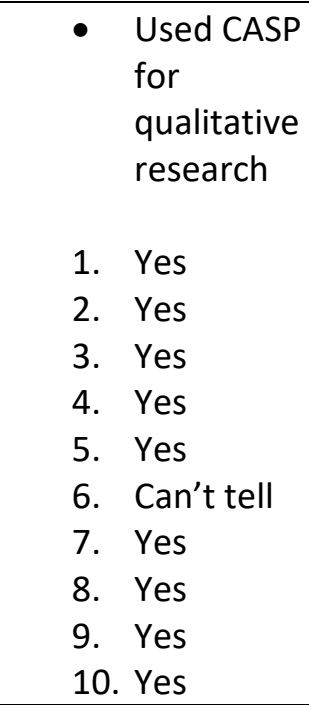 \\
\hline $\begin{array}{l}\text { Waldemar, A. K., } \\
\text { Esbensen, B. A., Korsbek, } \\
\text { L., Petersen, L., \& Arnfred, } \\
\text { S. (2018b). Recovery } \\
\text { orientation in mental }\end{array}$ & $\begin{array}{l}\text { Setting - Open, inpatient ward. } \\
\text { Data Collection - Semi-structured } \\
\text { interviews informed by the Recovery Self- }\end{array}$ & $\begin{array}{l}\text { - Used CASP } \\
\text { for } \\
\text { qualitative } \\
\text { research }\end{array}$ \\
\hline
\end{tabular}




\begin{tabular}{|l|l|l|}
\hline $\begin{array}{l}\text { health inpatient settings: } \\
\text { Inpatient }\end{array}$ & Assessment measure with 14 service-user & 1. Yes \\
experiences?. International & interviews. & 2. Yes \\
journal of mental health & Data Analysis - Content analysis. & 3. Yes \\
nursing, 27(3), 1177-1187. & & 4. Yes \\
& Location - Denmark. & 5. Yes \\
& & 6. No \\
& & 7. Yes \\
& & 8. Yes \\
& 9. Yes \\
& & 10. Yes \\
\hline
\end{tabular}

Table 5 - Papers included within the review

\subsection{Synthesising the Data}

This review used a thematic approach in order to organise and discuss the existing literature in relation to the two specific questions outlined above. The reason for undertaking a thematic approach was due to the fact RE aims to identify contextual factors, resources and responses that influence how interventions work, and the subsequent outcomes. Using these categories to guide my analysis of the existing literature enabled me to identify factors that could be used to inform the subsequent research project. The literature view will therefore be structured around the questions stated in section 3.1 .

Various definitions of recovery were articulated by participants within five of the papers included in this review. A common thread across the papers was the contradictory accounts between service-users and staff and between staff working in the same team (Aston \& Coffey, 2012; Cleary et al., 2013; Hungerford \& Fox, 2014; Coffey et al., 2019a; De Ruysscher et al., 2020).

One conceptualisation of recovery, as articulated in interviews by some nurses working in acute mental health settings, related to symptom improvement and medication adherence (Cleary et al., 2013), which suggested a medicalised and clinical view of recovery. Coffey et al (2019a) reported that some service-users also focused upon medication and symptom suppression within their descriptions of recovery. Coffey et al (2019a) proposed a potential reason for service-users articulating recovery in this way was 
because clinical recovery and medication is often the focus of inpatient care and therefore, they mirror this narrative within their accounts.

Mental health staff also considered recovery in terms of service-users getting better and recovering something lost (Aston \& Coffey, 2012). Some staff went further and suggested that recovery was about service-users returning to their frame of mind before being ill that is near to a normal life, across all aspects of their life (Aston \& Coffey, 2012; Cleary et al., 2013). These articulations by mental health staff construct recovery as problemfocused suggesting that a deficit and medicalised model was used to understand mental illness and make treatment decisions. Aston and Coffey (2012) suggested that these constructions of recovery positioned staff as the orchestrators of recovery, suggesting recovery is technocratic and mechanistic.

Some mental health staff used certain markers as indicative of recovery; these included service-users leaving hospital and having the ability to manage everyday tasks (Cleary et al., 2013). This suggested that some staff had an outcome-focused approach to recovery. This contradicts service-user accounts, from a focus group with 6 service-users, who articulated recovery as a journey or process that does not have a destination (Aston \& Coffey, 2012; Hungerford \& Fox, 2014). Service-user accounts, from focus groups and interviews, suggested that recovery is a dynamic process, that does not relate to a cure, but instead relates to a state of being (Hungerford \& Fox, 2014). This highlights that there were apparent differences between staff and service-user articulations of recovery, which alludes to there not being a consensus on what recovery means in practice.

Other service-users discussed their feelings of loss in all parts of their life as a result of their mental illness and described the need to consider what was lost, their rights, responsibilities, roles, and potential (Aston \& Coffey, 2012). Some staff accounts described the need to consider many facets of an individual's lives, such as education, money, work, relationships, psychological needs, practical matters, and living skills (Cleary et al., 2013), however this was not a common conceptualisation. This suggests some nurses were aware of the need to not solely focus upon an individual's mental illness but that services need to address the multiple areas of life that may be affected by mental illness. This highlights some convergence between staff and service-user accounts. 
Only one paper considered recovery as something different from clinical or personal recovery. De Ruysscher et al's (2020) paper, which involved interviewing both staff and service-users, suggested that recovery was a relational process that relies upon the interdependence between individuals, their social context and their relationships. This appears to differ from other articulations of recovery, as service-users were not positioned as needing to change or get better, and instead the role of others and wider society were considered. This proposes a model that considers the social determinants of mental health and barriers to recovery is necessary.

Coffey et al (2019a) reported that many staff participants, who were recruited from across 19 different mental health settings, were aware of the disparate meanings of recovery and viewed this disparity as problematic for inpatient contexts. As the construct of recovery is poorly understood it is unlikely to help inform care and treatment (Coffey et al., 2019a). Staff and service-user participants in focus groups (Aston \& Coffey, 2012) and PMs working in inpatient settings (Chisholm et al., 2020), believed that a shared understanding of recovery was necessary for ROC to be actualised.

This highlights that the conceptualisation of recovery and ROC is contested. This raises the question of how services can be delivering ROC when various stakeholders are defining recovery in varying ways. This also highlights that ROC within inpatient settings is complicated by challenges from the exosystem level which is characterised by a lack of homogeneity in what recovery and ROC is.

\subsubsection{What are the Barriers to Delivering ROC within the Context of Inpatient Services?}

Challenges relating to the incorporation of ROC within inpatient care were identified within all the papers included within this review.

\subsubsection{The Characteristics of Inpatient Settings}

A focus upon medication was seen within several papers. Interviews with service-users (Hungerford \& Fox, 2014) and stakeholder interviews (Chen et al., 2013) reported that medication-, task- and problem-focused approaches to care were adopted within inpatient settings. Non-adherence to medication was perceived to be a barrier to recovery 
by mental health nurses in inpatient settings (Cleary et al., 2013). It was observed in an ethnographic study that faster transfers to open conditions were offered if service-users complied with medication (Waldemar et al., 2018a). This suggests that great emphasis was placed upon the role of medication and compliance within inpatient settings. The proliferation of medication within mental health settings contrasts with ROC which encourages choice and recovery, which can include not using medication if the serviceuser desires (Chisholm et al., 2020). Despite the emphasis on medication, particularly in acute services, service-users proposed that their concerns about medication were not always addressed (Chen et al., 2013). This casts doubt upon whether they were involved in decisions about their treatment and medication.

It was suggested that staff were not well equipped in alternative treatments to medication, as the medical model was used as the pervasive framework to understand mental illness and solve any associated problems (Chen et al., 2013). This highlights that not only were inpatient services prioritising medication as the treatment focus, but they also did not have the skills to provide individuals with other forms of support, limiting treatment options. Whilst the majority of the papers identified there was a dominance towards medication within inpatient settings, some staff suggested they were attempting to shift away from medication adherence being a marker of recovery by providing education about mental illness, medication, side effects and recognising early warning signs (Cleary et al., 2013). However, there was still an emphasis on medication and mental illness as the central feature of inpatient settings.

Power imbalances between staff and service-users made it difficult for collaborative relationships to be developed and maintained, meaning staff dominated decision-making, limiting service-user autonomy (Chen et al., 2013). Decision-making and communication were top-down within inpatient environments, and there are no protected channels for service-users to have a voice or be involved in decisions (Chen et al., 2013). Staff reported that they were concerned about sharing power as they thought this might compromise professional boundaries and their authority (Chen et al., 2013). This highlights a potential reluctance to shift the decision-making processes from professional-led to collaborative which is expected within ROC. 
The restrictions imposed in inpatient settings were also identified as barriers to ROC. Wider factors that govern the delivery of inpatient services, such as compliance with legislative regulations (De Ruysscher et al., 2020) meant imposed rules prevented more tailored, individualised approaches from being delivered. Ward rules and routine meant service-users were restricted in what they could do, or required staff permission, leading to feelings of powerlessness and power imbalances (Chen et al., 2013). These findings suggest that the context of inpatient care may not be conducive with ROC, as serviceusers were not treated as equals, did not have an outlet to vent or express themselves, and there was a focus upon compliance as opposed to choice (Hungerford \& Fox, 2014).

The primary purpose of inpatient care, as cited within interviews with staff and serviceusers, was medication, stabilisation and the earliest possible discharge (Chen et al., 2013; Hungerford \& Fox, 2014; Waldemar et al., 2018a; Waldemar et al., 2018b). The short-term and custodial nature of admissions were noted, by staff, as a barrier to ROC due to the rapid turnover of service-users (Cleary et al., 2013), individual distress influencing their ability to engage and staff having limited time to get to know the person behind the illness (Coffey et al., 2019a). The nature and function of inpatient services appear to be at odds with $\mathrm{ROC}$ and raises the question of whether ROC is compatible in these settings.

\subsubsection{Implications of ROC in Inpatient Care}

When attempting to deliver ROC, service-users suggested that staff took a hands-off approach to care and they were left to their own devices (Hungerford \& Fox, 2014; Coffey et al., 2019a). Carer and service-user participants in Hungerford and Richardson (2013) and Aston and Coffey (2012) suggested that the hands-off approach was borne out of services shifting the responsibility for recovery onto service-users and carers, and away from services. The success or failure of recovery was dependent upon the service-user, which omits the role of services and the wider community in supporting mental health recovery (De Ruysscher et al., 2020).

The daily practice of inpatient services was influenced by macro-level factors which were claimed to inhibit the ROC vision. Staff participants in Cleary et al (2013) proposed that factors such as homelessness, social isolation and being from poor socioeconomic 
background were barriers to recovery. Stigma was also seen within the fabric of inpatient service delivery as stigmatising language was used by staff and within the community (Chisholm et al., 2020). Chen et al (2013) proposed the need for strategies to address stigma, discrimination and internalised stigma, such as collaborating with peer advocates and groups. However, these were recommendations presented by the authors (Chen et al., 2013) and not common practice within the services. It was suggested that inpatient settings afforded insufficient space to the wider structural and social issues that cause and maintain mental distress (Coffey et al., 2019a), meaning recovery and opportunities for recovery were hindered by the narrow focus of services.

De Ruysscher et al (2020) reported that service-users were often confronted with stigmatising experiences and their identity was narrowed down to their mental illness. The staff at the service needed to view the individual as someone with unique qualities, rather than reduce them to their diagnosis. Staff achieved this by engaging in a dialogue with service-users to identify their social roles and personal interests to connect them with other aspects of their identity, shifting away from their identity as an individual with a mental illness. This was further supported by staff's attempts to connect individuals with the community, such as finding accommodation and sourcing meaningful activities in the community (see section 3.3.2.4). These findings highlight a need for a broader view of recovery which considers wider socio-political factors rather than services focusing solely upon supporting changes within the individual; which was not being achieved by inpatient settings (Coffey et al., 2019a). This raises the question of whether services are expected to focus solely upon the individual or should also be responsible for the social change needed for service-users to be equal and participating citizens in society (Anthony, 1993).

\subsubsection{What Resources (Mechanism) are used to Deliver ROC in Inpatient Services, and What Outcomes are Associated with these Resources?}

Across the 14 papers included within this review, four areas of service delivery were identified as reflective of ROC. These four areas were staff, choice, care planning and community access and integration. 


\subsubsection{Staff}

The role of staff in delivering ROC was frequently discussed across the papers. Several staff qualities were identified as reflective of ROC. Staff, service-user and PM participants all identified that individuals need to be treated with care, compassion, honesty and integrity by all staff (Chen et al., 2013; McKenna et al., 2014; Coffey et al., 2019a; Chisholm et al., 2020). Findings from Coffey et al (2019a) suggested that service-user participants did feel they were treated with dignity, respect and compassion across the 19 different inpatient services. This highlights that the humanistic and caring side of treatment was reportedly being achieved across the various inpatient contexts. However, service-users in other settings expressed, in interviews, that some staff were cold, unapproachable, unsympathetic and disrespectful which made them feel inferior or as if they were being talked down to like a child (Chen et al., 2013; Waldemar et al., 2018a). This suggests that the attitudes and qualities of staff can be influential in the delivery of ROC, and recovery supportive attitudes and qualities vary between staff, and between services.

Coffey et al (2019a) reported that there was an association between ROC and the quality of therapeutic relationships and how service-users rated the quality of their care; meaning that when therapeutic relationships were scored highly the perception of quality of care was also scored highly. This further highlights the importance of staff being able to establish therapeutic relationships for ROC to be delivered. Osbourn and Stein (2019) reported that the working alliance between staff and service-users accounted for a significant proportion of the variation in individual's reported wellbeing and views of ROC. The findings of these papers show that the individual characteristics of staff and the way they interact with service-users can help or hinder the delivery of ROC. It emphasises the importance of recruiting staff who can work in caring, compassionate and humane ways within inpatient settings which supports the principles and delivery of ROC.

Quantitative data from Tsai and Salyers (2010) reported that hospital staff, when compared to community-based staff, had lower levels of personal optimism, service-user optimism and recovery-orientation. Inpatient staff were less optimistic about individual's ability to live independently, cope with their symptoms, achieve their goals and be actively involved in the community. The authors proposed that this could be due to inpatient staff 
only seeing service-users with the greatest needs, or those who are frequently readmitted. Chen et al (2013) suggested that inpatient staff who work with individuals with poor prognosis could become hopeless, which conflicts with the principles of ROC. This suggests that although staff can work in supportive and caring ways, staff attitudes towards recovery and the future for service-users may have the potential to influence ROC. It also highlights that inpatient settings may negatively influence staff attitudes relating to hope; this finding warrants further research attention, as the context of inpatient settings may not only be preventing the delivery of ROC, but may be hindering the ability of staff to work in recovery-oriented ways. Literature suggests that staff who are pessimistic might anticipate negative outcomes or perceive service-user's circumstances as bleak (Jackson, 2009), whereas clinician optimism has the potential to improve treatment satisfaction and outcomes (Byrne et al., 2007). This suggests that staff attitudes in relation to pessimism and optimism are influential elements of therapeutic relationships for recovery. This is particularly relevant given the fact that service-users wanted services and staff to be hope-focused and supportive to help them achieve their dreams (Hungerford \& Fox, 2014). Staff participants, in McKenna et al (2014), recognised that hope was crucial to starting the recovery journey, however, noted that involuntarily admitted individuals do not want to be there, nor do they want to talk to staff when first admitted. This meant staff needed to engage service-users in a way that did not impose upon or overwhelm individuals. Although hope was recognised as a key recovery process by staff participants, the context of inpatient care, at times, prevented hope from developing in both staff and service-users; for example, the context of being involuntarily admitted to a service. This highlights the complexities of delivering ROC in the context of inpatient settings.

Getting to know the individual was identified as a means of building rapport and trust which is crucial to the development of therapeutic relationships; however, due to a lack of time within acute care, staff had to rely upon opportune moments to engage in informal and passing conversations (McKenna et al., 2014). Staff reported that they did not have the time for informal conversations, despite service-users reporting these interactions were beneficial for developing trust and feeling equality between themselves and staff (Waldemar et al., 2018b). Aston and Coffey (2012) and Waldemar et al (2018b) found, 
through interviews with service-users, that staff were often busy, were occupying the office or were perceived to be doing nothing. This led service-users to feel alone, distanced from staff and missing the opportunity to talk about their feelings and experiences. Other studies have also noted that nurses direct face-to-face time with service-users was limited (McAndrew et al., 2014; Terry \& Coffey, 2019), and this was often due to working in complex environments characterised by competing priorities (Cleary et al., 2012). Additionally, inpatient care involved constant observations which created a barrier between service-users and staff (Waldemar et al., 2018b). This suggests that the environment of inpatient care and the demands that are associated with these settings, such as ensuring people's safety, may prevent ROC from being achieved in situations where individuals are detained against their will.

Despite the reported importance of the role of nurses in ROC, nurses reported that they did not know how to transfer their knowledge of recovery to inform the way they work (Chen et al., 2013) and they were uncertain of their role in terms of supporting recovery (Aston \& Coffey, 2012; Hungerford \& Fox, 2014). There was a sense that the concept of recovery was imposed on the nursing profession (Aston \& Coffey, 2012), that there was insufficient policy on how to deliver ROC (Cleary et al., 2013) and limited information on what was expected of staff (Hungerford \& Fox, 2014). This suggests that ROC was limited by a lack of awareness amongst staff of how to implement these principles into practice. Several papers noted that staff needed to change their role and how they support people in their recovery (Hungerford \& Fox, 2014; Waldemar et al., 2018b; Chisholm et al., 2020; De Ruysscher et al., 2020). Service-users wanted staff to adopt a role of guiding them in their recovery and wanted to have space to learn, try new things, negotiate with staff and not be punished for setbacks (Waldemar et al., 2018b; De Ruysscher et al., 2020). In ROC staff are expected to focus upon skills as opposed to deficits, by identifying service-users' hobbies and ambitions, and reframing setbacks as learning opportunities for personal growth (Chen et al., 2013; McKenna et al., 2014); however, there was staff resistance or lack of motivation to change, meaning the medical model remained the dominant framework within inpatient services (Chen et al., 2013; Hungerford \& Fox, 2014).

Staff and service-users in acute inpatient care expressed the expectation that ROC was the responsibility of community or outpatient services (Hungerford \& Fox, 2014; 
Waldemar et al., 2018b), which created a sense of uncertainty of who is responsible for ROC and which services should be delivering it. Some service-users suggested that in acute settings the biomedical approach had been strengthened by the introduction of ROC, as this gave rise to reliance upon community services to provide psychosocial care and support, whilst inpatient services focused upon medication and stabilisation (Hungerford \& Fox, 2014).

\subsection{Peer Mentors}

PMs were identified as leaders in the delivery of ROC and create the culture needed for ROC to be achieved (Chen et al., 2013; Hungerford \& Fox, 2014; Chisholm et al., 2020). Despite PMs being recognised as potential leaders in the delivery of ROC, several barriers were identified in practice within the only paper that included PMs as participants (Chisholm et al., 2020).

In a focus group with 8 PMs, participants reported that some staff were ignorant towards the value of lived experience which was a barrier to PMs delivering and leading ROC within inpatient service (Chisholm et al., 2020). PMs identified power imbalances between staff and PMs working in inpatient settings, which further prevented ROC from being delivered. PMs felt discriminated against, not listened too by mental health professionals and stigmatised. This highlights that there was a lack of understanding of the role of PMs, and how lived experience could be beneficial in delivering ROC, but also some resistance to their inclusion.

PMs suggested there was not enough funding to employ the number of PMs needed to shift the culture towards ROC, suggesting that PMs were having limited influence over service delivery in their current numbers (Chisholm et al., 2020). This highlights that although the inclusion of PMs as part of service delivery is in keeping with ROC, simply adding PMs to the workforce does not, by itself, create the paradigm shift needed for ROC to be delivered in inpatient settings. It also evidences the need for wider support for inpatient services to be able to include PMs in a meaningful way, which would mean additional resources need to be available. It should be noted that the challenges of PMs having a role in the delivery of ROC was based upon one paper, and therefore caution is advised regarding the generalisability of these findings. 


\subsubsection{Choice}

Choice was identified as one aspect of care that was reflective of ROC. Service-users wanted to be supported to make their own choices, have control over their choices and be made aware of the options available to them (Hungerford \& Fox, 2014). It is, however, important to firstly consider the context of inpatient care when discussing choice.

In acute contexts many service-users accessed the service under circumstances of legal coercion, meaning they had little choice over their admission (McKenna et al., 2014). If people were in crisis or experiencing acute episodes of mental illness, staff presumed service-users decision-making abilities had been compromised by their illness and therefore limited service-user opportunities for autonomy and made decisions on their behalf (Chen et al., 2013). This suggests that service-user choice could be removed by staff, which further evidences the power imbalances in inpatient settings (Chen et al., 2013). The fact that mental health staff can use coercion challenges the notion of an equal partnership, collaboration and that service-users have choice (Waldemar et al., 2018a). In acute mental health settings, people were encouraged to engage in compulsory treatment by staff, which suggests service-users did not have a choice regarding treatment, or whether they engaged in such treatment (McKenna et al., 2014). McKenna et al (2014) suggested that service-users have little choice as they are detained, therefore staff present the illusion of choice, such as whether medication is taken orally or injected, but they had no choice of whether they take medication. This raises the question of whether ROC can be achieved in settings where coercion and restrictions are a significant feature of care. This highlights the importance of considering context in the delivery of ROC. For individuals who have had their liberty removed and have been involuntarily admitted to hospital, any choice offered is limited by the circumstances that the individual and the staff at the services find themselves in.

In addition to the limited choice, an ethnographic study, which captured the day-to-day delivery of ROC, found that choice was rhetoric, and whilst individuals voiced their opinions these did not inform practice or the subsequent decisions made (Waldemar et al., 2018a). Service-users reported that their contribution to treatment decisions was largely accepting or declining different medical options and reporting back to staff the 
associated side-effects (Waldemar et al., 2018b). Waldemar et al (2018b) noted that although individuals had the option of declining medical options presented by staff, if they did this, it was deemed ill-considered by staff. This suggests that service-users may be provided with a choice, but staff judged these decisions. As this was reported within an ethnographic study, this would suggest that staff judgements and views on staff's preferred course of action are implicitly or explicitly communicated to service-users, indicating to them that a certain choice is more desirable. This suggests that the process of socialisation to norms operates within mental health inpatient settings, and serviceusers are expected to learn and go along with these norms and values (Arnett et al., 1995). Additionally, some expressed, and were observed, to be perceptive towards staff's ability to determine the right treatment and would, therefore, allow decisions to be made on their behalf by staff (Waldemar et al., 2018a; Waldemar et al., 2018b). The reason why service-users behaved in this way were not explored in these papers, but it does suggest there was variation in whether they made decisions for themselves, or whether these decisions were what staff saw as advisable.

The one area that service-users appeared able to negotiate more with staff was how they spent their free time, such as utilising leave, and what leisure activities they engaged in (Waldemar et al., 2018a; Waldemar et al., 2018b). Despite service-users supposedly having these choices, Chen et al (2013) found that inpatient services were often bereft of activities, resulting in boredom, which raises the question of whether choice relating to leisure activities was actually facilitated in inpatient settings. An ethnographic study also reported that service-users needed to ask staff for permission and request to do things and found that individuals who were more subdued received more support and permission from staff (Waldemar et al., 2018a). This suggests that there were inconsistencies in how staff supported choice in practice, and further highlights a power imbalance between staff and service-users. Inconsistencies were further evidenced by some staff prioritising meeting individual's needs, and others prioritising the rules and rigidity of the ward when responding to choice and needs (Waldemar et al., 2018a). This suggests that although service-users appeared to negotiate more regarding leisure choices, staff attitudes influenced whether these choices were facilitated, indicating that choice ultimately rested with the staff. 
Despite choice being acknowledged and presented as part of ROC, staff acknowledged that there was a paradox of offering choice in situations when in fact there is no choice (Waldemar et al., 2018a). There were numerous tensions and ethical dilemmas that arose within the inpatient context that influenced how staff supported choices. There were tensions between maintaining the order, safety and stability of the ward and promoting human rights of service-users, such as choice (Chen et al., 2013). This was particularly enhanced when staff were presented with the dilemma of supporting service-user choices that might have harmful, risky or bad outcomes (Chen et al., 2013; Gwinner \& Ward, 2015). There is a professional responsibility placed upon those working in inpatient settings which creates an opportunity for staff to be blamed if choices are made that have harmful or bad outcomes, therefore staff tended to be more risk-averse, which would limit opportunities for service-users to make choices, learn and take responsibility (Chen et al., 2013). Staff also reported difficulties when discussing care with service-users when there was a mismatch in goals and expectations (Coffey et al., 2019a). This highlights uncertainty on how staff should support recovery when choices may not be supportive of recovery or align with the professional judgement of staff. Staff were also uncertain of how to support individuals with apathy or low motivation as they note that there is a fine line between coercion and engaging individuals (Chen et al., 2013). This highlights that there are challenges for staff when service-users are under the care of services, as there is a duty to maintain their safety, but also to promote choice and how to balance these two occasionally competing rights appears complex.

Lastly, inpatient settings also experienced demands from external agencies, such as the need for quick discharges for improved bed capacity; meaning service-users experienced intense persuasion from staff to take leave or early discharge (Waldemar et al., 2018a). This was noted as inhibiting individual's opportunities to exercise their right to choice and appeared to conflict with the vision of ROC. This highlights that there are external demands placed upon inpatient services, and these demands, particularly if financial, may be prioritised above the delivery of ROC. 


\subsubsection{Care Planning}

Care planning was identified as a practical means of delivering ROC as this provided an opportunity for collaboration between staff and service-users. Coffey et al (2019a) found that staff suggested that care planning occurred with the involvement of service-users and the multidisciplinary team to manage the transition from inpatient services to the community. However, in the same research service-users reported that they were not involved in the process of care planning, did not know the content of their care plans, did not have a physical copy, nor did they feel a sense of ownership over their care plans. These diverging accounts regarding service-user involvement in care planning was reported across all 19 services included in Coffey et al (2019a). The lack of service-user involvement in care planning was further supported by findings from an ethnographic study which reported that individuals were vague about the goal of their admission, lacked information regarding their treatment, did not know what their treatment consisted of, or the rationale for this treatment (Waldemar et al., 2018a). Staff were also prone to making interpretations about service-user needs which at times resulted in their requests, questions and needs being dismissed. Furthermore, there were occasions when decisions would be made before consulting the service-users (Waldemar et al., 2018a).

The communication and information sharing relating to service-user treatment and care planning was also acknowledged as challenging (Hungerford \& Richardson, 2013; Coffey et al., 2019a; Waldemar et al., 2018b). It was reported that a lack of shared language regarding recovery and treatment was a barrier to service-user involvement (Coffey et al., 2019a), and they reported confusion as they were provided contradicting advice from the clinical team (Waldemar et al., 2018b). In addition to the findings that service-users were not involved in decisions about their care, Coffey et al (2019a) reported that there was an unwillingness amongst some service-users to collaborate and contribute to care planning. The authors attributed this unwillingness to data which suggested that they did not see care plans as an integral or important component of their experience. Service-users had little involvement in the care planning process, and therefore the authors argued that service-users have learned over time that, in many cases and despite the rhetoric of care planning, these plans do not really help them. In another study, staff reported that 
collaborative care planning was not imposed upon service-users due to their potential inability to engage, as a result of their acute distress (McKenna et al., 2014). The findings from Coffey et al (2019a) and McKenna et al (2014) suggest that some individuals may not have wanted or were not able to be involved in this process, suggesting lack of serviceuser involvement may have not solely been due to being excluded by the service and staff. Analysis of service-user interview data showed that staff took charge of treatment decision-making and determining the suitability of their goals (Waldemar et al., 2018b). These findings, however, suggest that service-users may have been on the periphery of decision-making, or completely excluded from this process within inpatient care.

In addition to service-users reporting that they were not involved in decisions about their care, the nature of inpatient settings and the individuals receiving treatment there were identified as barriers by staff participants. Staff working in acute settings reported that the short-term nature, high turnover of service-users and scarcity of time to establish collaborative relationships were barriers to care planning (Chen et al., 2013; Coffey et al., 2019a). This highlights that the function and organisational logistics of inpatient care, particularly acute inpatient settings, could impinge upon collaborative care planning. An ethnographic study reported that treatment planning was also influenced by the demands of external authorities, which meant other demands, such as bed capacity and the need for high rates of discharge, competed with whether collaborative care planning occurred (Waldemar et al., 2018a). This highlights that exosystem factors influenced the delivery of ROC in inpatient contexts. This suggests that service operation, as well as wider structures, such as government pressures, influenced how collaborative care planning was achieved in practice.

The involvement and inclusion of family and carers was also recognised as key to collaborative partnerships (McKenna et al., 2013). Despite this, interview data suggests that three out of ten carers were not aware of ROC, and many felt excluded (Hungerford \& Richardson, 2013). Carer participants also reported feeling that there was a lack of collaboration and information sharing between the team and themselves regarding service-users mental state, risk, needs, treatment or progress (Hungerford \& Richardson, 2013). Carers attributed the cause of this insufficient communication to staff incorrectly assuming carer groups and advocates provided carers with this information. However, 
carers wanted personalised and tailored information relating to the service-user. Carers found themselves questioning the appropriateness of the treatment options if there is limited information sharing between carers and the clinical team. Carers also identified the legislative restrictions surround confidentiality as preventing carer involvement and collaboration, leaving them feeling marginalised, devalued and dismissed (McKenna et al., 2014).

\subsection{Personalisation, Holism and Skills}

Some service-users identified that having flexible and individualised care was for them reflective of recovery (Hungerford \& Fox, 2014). Care planning was identified as needing to be personalised, individual and holistic, meaning various aspects of service-user's lives were supported and addressed.

One service attempted to achieve this by ensuring care was tailored to the individual and pre-defined activities were not imposed upon service-users (De Ruysscher et al., 2020). Staff encouraged individuals to formulate what they expect from their admission and how the service could support their recovery, meaning service-users were taking back agency over their life (De Ruysscher et al., 2020). The authors acknowledged a potential danger of individualised approaches to recovery as it risks service-users being exclusively seen as individuals who are responsible for the success or failure of their therapeutic trajectory and a neoliberal mindset. This was the only paper to explicitly consider the political influence of how recovery has been adopted by services. Chen et al., (2013) did, however, suggest that biomedical, psychological, social and socio-political models were required to support individuals in their recovery, however, this was rhetoric and no findings were presented relating to how this was being achieved in routine practice.

One paper referred to holistic care which meant incorporating social, cultural and religious needs into the care and treatment of individuals to ensure the diversity of the service-users were catered for (McKenna et al., 2014). Examples of holistic care were identified, these included sensory modulation, music therapy, perfumed oils, weighted blankets and staff being flexible in the medication and mealtimes during Ramadan, which was easier if the staffing team were diverse and had knowledge of different cultural or 
religious needs (McKenna et al., 2014). However, the facilitation of holistic care was influenced by the lack of time for staff to support these alternative forms of treatment.

Chen et al., (2013) and McKenna et al (2014) referred to the need for inpatient services to provide people with information and skills, such as daily living, social and vocational skills, to manage their illness. Community participation and citizenship were associated with life and social skills (Chen et al., 2013; McKenna et al., 2014). A lack of skills was identified as impeding service-users' social participation (Chen et al., 2013), therefore learning or relearning skills were considered necessary for community life. For example, across the 5 inpatient services in McKenna et al (2014), service-users were expected to tidy up, do laundry and prepare food. Recovery and citizenship appeared to be conflated with service-users fitting in or becoming independent of support or services; as opposed to creating inclusive communities or having the appropriate support and adjustments to ensure full and equal participation within society.

\subsubsection{Community Access and Continuity of Care}

Ensuring that service-users were not disconnected from the wider community was also identified as a central component of ROC within inpatient services. Two aspects relating to the wider community were identified. One was keeping the outside alive by supporting service-users to find a place and role within the wider community whilst they were still in inpatient services. The second related to the continuity of care between the service and community (McKenna et al., 2014; De Ruysscher et al., 2020). Despite continuity of care being a cornerstone of modern mental health (Schultz \& McDonald, 2014), there is a lack of consensus of how continuity of care is defined (Weaver et al., 2017). In these papers, continuity of care referred to providing a seamless continuity between life 'inside' the service' and 'outside' in the community, and ensuring service-users had access to opportunities to develop their identity and position in the community (McKenna et al., 2014; De Ruysscher et al., 2020).

Admission to an inpatient service was recognised by staff participants as being a major disruption to service-users lives (McKenna et al., 2014) and therefore some staff participants highlighted the need for seamless continuity between time spend in services and having a valuable community life (De Ruysscher et al., 2020). Participants noted that 
recovery takes place within society where service-users are participating as citizens. Services attempted to put measures in place to improve the continuity between inpatient care and the community.

One practical attempt by inpatient services to improve the continuity of care between hospital and the community was identifying and inviting the case manager to visit the service-user whilst the individual was still admitted to try to build rapport and ensure treatment continued in the community (McKenna et al., 2014). However, the most commonly cited means of keeping the outside world alive was encouraging service-users to engage in community-based activities (De Ruysscher et al., 2020). The provider encouraged community-based activities by making the activities at the service optional, in the hopes service-users would be more inclined to engage with opportunities outside of the service. Involvement in community-based resources and support was associated with individuals identifying their social role, as well as forming and maintaining connections with families, friends and social resources that could help support individuals upon discharge (Chen et al., 2013). Staff actively searched for welcoming opportunities in the community so that the boundaries between inside and outside became more blurred and porous for both staff and service-users (De Ruysscher et al., 2020). However, opportunities to participate as a citizen were restricted or limited to spaces that welcomed individuals with a mental illness (Chen et al., 2013; De Ruysscher et al., 2020), which could be viewed as a form of ghettoisation. Quantitative survey data also showed that few inpatient staff felt that service-users were helped to build community connections or achieve goals such as educational or employment aspirations (Tsai \& Salyers, 2010). This suggests that although attempts were made to support individuals to access community-based opportunities, these were restricted or faced challenges, which raises the question of whether this helps service-users to become equal citizens in society. Despite the aspirations of some providers and staff to help service-users find a meaningful place outside of the inpatient service, it was not without its challenges. Some individuals were scared of thinking about recovery as this would mean behaving in certain ways, such as getting a house and car (Hungerford \& Fox, 2014). Focusing upon life outside of hospital meant that some long-stay service-users were confronted with a new set of expectations, as staff claimed service-users had become accustomed to life inside services and could no 
longer envisage feeling at home elsewhere (De Ruysscher et al., 2020). The reason for this staff view was because service-users were not having their basic needs met outside of services for example, there were high levels of homelessness amongst people accessing one inpatient service (De Russycher et al., 2020), whereas they were having these basic needs met in inpatient services. This highlighted that the issues some service-users were facing when in the community where no longer applicable when in services, and some staff participants suggested that some became accustomed to having their basic needs met by services, which some staff participants claimed could influence service-users motivations for wanting to leave services. Some staff participants suggested that by providing a safe, welcoming environment this has the potential to install a false dichotomy that the ward is safe, and the outside world is threatening. This highlights that staff must balance ensuring service-users feel safe and supported in inpatient settings, which is the idea of an asylum, with ensuring they feel there is possibilities outside of services, which is the idea of rehabilitation. Inpatient care needs to do both, safety and stabilisation initially and then progressing towards return to community living and some form of independence.

Service-users, staff and carers all recognised the need for greater collaboration between inpatient settings and community services to improve continuity of care (Cleary et al., 2013; Hungerford \& Richardson, 2013; Hungerford \& Fox, 2014; De Ruysscher et al., 2020). Carer participants suggested that there were limited community-based services to support service-users upon discharge, meaning the responsibility to provide support was placed upon carers, hence they did not have access to the resources needed to achieve their goals (Hungerford \& Richardson, 2013). Interview data from nurses showed there was insufficient follow-up upon discharge which was a barrier to recovery (Cleary et al., 2013). These findings highlight that the coordination between inpatient services and community-based care was insufficient and there were limited resources to support service-users in their recovery. This shows that some participants recognised that recovery is an ongoing process and individuals may require continued support, and that inpatient care only forms part of the recovery journey. However, this ongoing process and continued support does not appear to be reflected within an established care pathway between inpatient and community services. 
Staff were aware of the need to consider multiple aspects of an individual's life upon discharge, such as education, housing, relationships and finances, which was in keeping with the holistic view of recovery previously discussed (Chen et al., 2013) (see 3.3.2.3.1). However, the service's ability to support service-users in all these areas of life upon discharge were influenced largely by external organisations (De Ruysscher et al., 2020). The example of social housing was used to reflect the challenges services faced when attempting to improve the continuity of care between inpatient services and the community. Social housing has long waiting lists and some service-users experienced stigma preventing them from accessing housing. This meant that inpatient services did not have control over the search for housing, which prevented ROC from being delivered at the service (De Ruysscher et al., 2020). The external agencies which inpatient services try to work alongside to promote ROC, may not operate, or understand mental health recovery in the same way, meaning these collaborations were not operationalised effectively.

The same service also provided an aftercare programme which aimed to be as short as possible so service-users could increasingly find more meaningful roles and activities within the community (De Ruysscher et al., 2020). However, this was again impeded by external partners, such as housing organisations, who would use the aftercare programme as a prerequisite for securing a place at a housing unit. This meant that service-users would have to use the service, as opposed to establishing meaningful connections and activities within the community, to secure housing (De Ruysscher et al., 2020). To counteract these issues staff provided support and coaching to communitybased organisations, such as social housing, and the informal support network of the service-user, such as family and friends (De Ruysscher et al., 2020). De Ruysscher et al's (2020) paper related to an inpatient service in Belgium and therefore the cultural and systematic issues of Belgium need consideration. It has been suggested that Belgium has a fragmented mental health sector due to a range of factors such as funding disparities, the division between health and social care, and interagency divisions (Lorant et al., 2016). To address this problem, the government has chosen to shift its mental health system towards community care, where individuals are treated in the community as opposed to hospital or institutional environments (Lorant et al., 2016). These challenges are not 
unique to Belgium, and have been reported within numerous European countries, however, awareness that this service is based in Belgium ensures important contextual issues are not overlooked. This finding reported in De Ruysscher et al (2020) suggests that inpatient services do not operate in isolation and rely upon external organisations to support service-users in their transition into the community and their recovery.

As the current ROC literature stands the connections between inpatient settings and community-based support do not appear to be well-established. This means that ROC may only be delivered to a certain degree, as the resources needed upon discharge may not be available, or service-users may encounter difficulties when attempting to access them. Social determinants and external agencies appear to influence service-user recovery and participation in the community; and inpatient services face similar challenges when attempting to address these barriers, such as sourcing appropriate housing (McPherson et al., 2018), however these appear to be at varying levels of success.

\subsection{Conclusions}

This literature review examined ROC within the context of inpatient mental health settings to understand what resources of ROC have been implemented within practice and what are the associated outcomes, and whether there were any barriers to ROC within the context of inpatient settings. Before addressing these two review questions, I noted that recovery and ROC were defined in numerous ways within the literature. The lack of homogeneity in how recovery and ROC is defined appears to pose a significant challenge for how services and staff operationalise recovery. A commonality across the papers was that recovery is idiosyncratic, if this is the case then this raises the question of whether ROC can be standardised for service delivery, or if this is a deviation from the true meaning of recovery.

Delivering ROC within the context of inpatient settings was not without its challenges. The function of inpatient care has become to stabilise and discharge as quickly as possible, meaning the nature of these settings are at odds with ROC. The papers included within this review noted that medication remains the dominate focus of inpatient care, and the proliferation of medication is often coupled with compliance meaning service-user choice and autonomy was dismissed and power imbalances between staff and service-users 
were prevalent. Staff also lacked the training to provide alternative forms of treatment other than medication meaning the shift towards ROC was restricted by the skills of the workforce. The individuals who were accessing the services included within this review have also experienced stigma, homelessness, social isolation and were from poor socioeconomic backgrounds meaning the individuals receiving care in inpatient settings may have several social determinants associated with their mental health. This highlights a challenge services face when they attempt to promote recovery, with these attempts potentially hindered once service-users are outside of services due to societal barriers.

The second review question asked what resources were delivered as part of ROC in inpatient settings, and what outcomes were associated with these resources. The reason for this question was to understand how services had attempted to operationalise recovery and ROC, and to inform this research project when identifying resources relevant to Woodlands. Four areas of service delivery were identified - staff, service-user choice, care planning and community access and continuity of care.

Staff who are compassionate, respectful and treat service-users equally were associated with the development of therapeutic relationships, trust and getting to know the person behind the illness. Despite the importance of staff, inpatient staff were reportedly less optimistic compared to community-based staff and were unsure of their role in delivering ROC. Practical attempts by staff to deliver ROC were identified, such as getting to know the individual behind the mental illness, however these were restricted by the lack of time available to staff and the nature of inpatient care to discharge as quickly as possible. The environment and demands of inpatient settings could prevent ROC from being delivered, meaning that despite the presence of compassionate, person-centred qualities and skills of staff, staff could not deliver ROC as expected.

Service-user choice was identified as central to ROC, however the context of inpatient care which is characterised by coercion, acute episodes of mental illness, power imbalances and restrictions on liberty, meant that the facilitation and promotion of choice was limited. This suggests that choice remained rhetoric within these services, with decisions ultimately resting with staff. This raises the question of whether inpatient settings will ever truly be able to support choice. 
Although care planning and the involvement of service-users in decisions about their care was identified by staff as a practical means of delivering ROC in inpatient settings, serviceuser and carer accounts suggested that care planning was not as successful as staff claimed. Service-users and carers felt excluded, did not know what was in care plans or did not have access to these documents. This highlights that there is a discrepancy in the value of care planning between staff and service-users, which suggests that this resource is not working as intended within the confines of inpatient care.

Lastly, access to the community and continuity of care between inpatient settings and the community were identified as central to ROC. Although staff participants recognised that an inpatient admission was a major disruption to individuals lives, services attempts to support integration in the community were influenced by several factors. As some serviceusers basic needs were not being met prior to admission, due to factors such as homelessness, poor socioeconomic backgrounds, and unemployment, services had to find the right balance between providing a safe space for individuals inside and promoting a life outside of services within the community. However, the attempts by services within this review to find community-based opportunities were restricted to opportunities that welcomed individuals with a mental illness, which could be viewed as a form of ghettoisation and prevented service-users from being active and equal citizens in society. This highlights the challenges services faced when attempting to support individuals to find a meaningful role outside of the service and evidences that services do not operate in isolation and are very much influenced by exosystem and macrosystem factors which can impede ROC from working as intended.

To conclude, this review shows that the evidence base for ROC within inpatient care is limited, and this is further limited within long-stay inpatient services. This highlights the need for future research into how ROC is delivered within the context of long-stay services, which this thesis considers.

\subsubsection{How Does This Review Link with the Overall Thesis?}

By answering these review questions, I will be able to apply my learning of how the context of inpatient care influences key recovery resources and the subsequent outcomes for service-users. This is advantageous as the service under evaluation is an inpatient 
service attempting to deliver ROC, and RE requires the consideration of context and how this influences outcomes. The benefits of this review meant that I could:

- Develop 'if then' statements relating to how ROC does, or does not work in inpatient settings;

- Identify outcomes of interest within the literature that would become outcomes of interest for this project.

From this review I developed 29 'if then' statements relating to the key resources associated with ROC within inpatient contexts (see Appendix II). I was mindful that the development of these 'if then' statements were based upon literature regarding services that were predominately providing acute care, whereas Woodlands provides longer-term care. Due to this, I did not develop these 'if then' statements into initial programme theories that would then be directly tested at Woodlands because I was aware that there would be several differences, such as the aim of these services, the interventions on offer and the demographics of the individuals accessing the service. I did, however, use these 'if then' statements to identify outcomes of interest, which will then be used within this research project. The outcomes identified from this literature review have informed the outcomes of interest for this project, which are:

\section{Staff Outcome 1 - a therapeutic relationship developed between staff and the service} user.

- Example 'if then' statement - If staff spend the time to get to know the individual behind the mental illness (resource) then this builds rapport and trust with serviceusers (response) and develops a therapeutic relationship (outcome). However, staff often have a lack of time to engage in these conversations due to competing priorities (context), leaving service user's feeling alone (response) and with limited opportunities to talk to somebody (outcome).

- Mechanisms of potential interest:

- Service-users feel they are treated with care, compassion, dignity and respect

○ Development of hope

- Development of trust between staff and service-users 
- Staff spend time getting to know the individual behind the mental illness

- Staff are cold, unapproachable and disrespectful - this has the potential to block this outcome from occurring

- Staff do not have the time to engage in informal conversations with service-users - this has the potential to block this outcome from occurring.

Staff Outcome 2 - peer mentors establish themselves as a different type of support (service level outcome) and service-users benefit from engaging with individuals with lived experience (individual level outcome).

- Example 'if then' statement - If PM are employed in mental health inpatient settings (resource), then there needs to be wider organisational support through staff understanding the role of PM and how lived experience could be beneficial for service-users (context), meaning the role of PM can be established as distinct and valuable by all at the service (outcome).

- Mechanisms of potential interest:

- Ongoing organisational support for the inclusion of PM

- Staff accept the role and value lived experience can have for service-users

- PM and service-users connected over similarities from their lived experience

- Power imbalances between staff and PM - this has the potential to block this outcome from occurring.

Choice Outcome - service-users make choices for themselves (individual-level outcome) and staff support service-users in the choices they make (service-level outcome).

- Example 'if then' statement - If staff prioritise meeting individual's needs (context) then they were move likely to support their choices (outcome), whereas if staff prioritise the rules of the ward (context) they are more likely to be risk-adverse when supporting service-user choice (outcome).

- Mechanisms of potential interest:

- Staff support service-users to identify what they need from services to support them to make choices 
- Provided with opportunities and support from staff to learn/relearn how to make choices for themselves

- Staff assume service-users decision-making abilities have been compromised due to service-users mental illness - this has the potential to block this outcome from occurring

- Staff are risk-adverse - this has the potential to block this outcome from occurring.

\section{Community Access Outcome - service-users access and engage in the community-based opportunities of their choice.}

- Example 'if then' statement - If service-users are involved in opportunities outside of the service (resource), then they can identify their social role and form and maintain connections with family, friends and social resources, which will be beneficial upon discharge (outcome). However, if these opportunities are only limited to spaces that welcome those with a mental illness (context), then this could promote structural violence and ghettorisation (outcome).

- Mechanisms of potential interest:

- Service-users have a desire and the confidence to explore opportunities outside the service

- Organisational/Staff commitment to identifying and supporting serviceusers to access community-based opportunities

- Community-based opportunities are available and accessible - this has the potential to block this outcome from occurring

- Service-users are fearful/scared of thinking of life outside of services - this has the potential to block this outcome from occurring

- Service-users become accustomed to being in services - this has the potential to block this outcome from occurring.

This research project will therefore focus upon the staffing group with consideration given to $\mathrm{PM}$, as well as service-user choice with consideration given to these choices in relation 
to community-based opportunities. There will also be scope to consider aspects of service delivery that programme architects identify as important to service delivery at Woodlands. In the next four chapters the data from this research project will be presented and analysed. The findings are presented in four chapters:

- The Wider Mental Health System

- The Staffing Model: Therapeutic Engagement

- Service-user Choice and Responsibility

- Quantitative Case Studies

The chapters are ordered using the EST model, moving from the wider mental health system to the direct environment of the service-users and their individual characteristics. The first findings chapter (Chapter 4) concentrates on Woodlands establishing and embedding itself as a new service within the pre-existing mental health system, with a specific focus upon referrals. The chapter explores if and how Woodlands has established itself with the locality of Wales and to what extent. Chapter 5 moves on to discuss CMOCS related to the staffing model used at Woodlands, with a specific focus on staffing qualities, staffing levels and the inclusion of peer mentors. Chapter 6 moves on to discuss another component of service delivery at Woodlands in the form of choice and responsibility. The chapter explores the challenges of finding the right balance between promoting autonomy and choice and ensuring service-users have the appropriate support in place in order to learn the skills to become more independent. The final findings chapter presents service-user case studies using routinely collected data from Woodlands with the aim of identifying patterns in the trajectories and experiences of individuals at Woodlands. 


\section{Findings - The Wider Mental HeAlth SYSTEM}




\subsection{Introduction}

Pawson and Tilley (1997) indicated that RE should reflect the embeddedness of an intervention within a stratified social reality. This means accounting for how both macro and micro processes influence interventions within practice (Shearn et al., 2017). To ensure macro and micro-level processes are considered I used an ecological perspective (see section 2.4.1). This allowed me to structure my analysis in a way that reflects the multiple connected levels that make up the contextual topography of Woodlands. The findings chapters will be presented by moving from broader constructs, such as the mental health referrals system to more specific constructs, such as service delivery at Woodlands.

In this chapter, I present my qualitative analysis of research interviews with PAs, staff, service-users and commissioners relating to the wider context the service operates within. This chapter will relate specifically to the exosystem of which Woodlands was operating within, and I will consider the interrelationship this system had with the microsystem and at the individual level of service-users and staff. The outcome of interest in this chapter relates to whether Woodlands was able to secure referrals and establish itself as a new provider.

This chapter with be followed with further analysis where I will examine what was said by participants about the role of staff, service-user choice and responsibility and finally my analysis of routinely collected service-level data.

\subsection{Brief Context of Woodlands}

Woodlands is a mental health service that offers 16 private en-suite bedrooms providing locked facilities and daytime psychological intervention and living skills training to support recovery (see section 1.2 for further information about Woodlands, which adds context to the findings of this chapter). 


\subsection{Interview Participant Details}

For the purposes of this thesis, I have classified participants based upon their position at Woodlands.

\subsubsection{Programme Architect (PA)}

Four individuals were identified by the gatekeeper as being involved in the design of Woodlands. All four agreed to participate and held senior positions within the wider organisation who ran Woodlands. PA research interviews ranged from approximately 50 minutes -2 hours in length. Three took place in the training room at the organisation's Head Office, and one took place in a conference room at Swansea University as this was more convenient for the participant.

\subsubsection{Staff}

Ten staff members were identified by the gatekeeper, nine agreed to participate and one potential participant passed away prior to recruitment commencing. Two participants were trained healthcare professionals, five were peer mentors with lived experience of a mental illness, one was a recovery practitioner, and one held an administrative role. I decided to not differentiate between the role's participants held in my reporting of data extracts in this thesis to preserve their anonymity. Staff research interviews ranged from approximately 30 minutes -2 hours in length.

\subsubsection{Service-users}

Eight service-users were identified and approached by the gatekeeper, and all eight agreed to participate. No service-users were excluded from participating using the exclusion criteria (see section 2.5.2.3). Service-user research interviews ranged from approximately 15 - 50 minutes in length.

I use the term service-user to refer to individuals who were accessing treatment at Woodlands. Some participants may refer to these individuals as 'guests' within data extracts as this was the term adopted at the service. However, I made the decision to use 
the term service-user based upon its use more broadly across literature and practice settings.

\subsubsection{Carers}

No service-users opted to nominate a carer that I could approach regarding participation in a research interview. The reasons given by service-users for not wanting to nominate a carer included: service-users not having anyone to nominate; having concerns about potential ramifications to their care at Woodlands, and concerns about placing unnecessary burden on their carer's current poor health. It should be noted there was only a limited available pool of carers given the number of service-users at Woodlands during the lifetime of my study. Due to these reasons a carer perspective was not be included (see section 8.5.4 where this is considered as a limitation of the project).

\subsubsection{Commissioners}

Three individuals were identified by the National Collaborative Commissioning Unit, as commissioners who had made referrals to Woodlands. All three agreed to participate in a research interview, which lasted approximately 30 - 50 minutes. These interviews were conducted by telephone and were audio recorded.

\subsubsection{Analysis}

I analysed PA, staff and service-user data using framework analysis (Ritchie \& Spencer, 1994), (see section 2.7.1). I identified nineteen initial descriptive categories from PA data. These initial codes were then refined into a framework which comprised of seven overarching categories relating to what PAs considered the key values of Woodlands' service delivery (see Appendix T). The categories were: the wider context, the staffing group, responsibility, choice, the intended service-user group, the physical environment and the wider organisation.

The framework was then used to code all staff and service-user transcripts. Within the categories, I would then subcategorise the data extracts relevant to each category to identify outcomes, responses and contextual factors, which contributed to the development and refinement of CMOCs. 


\subsubsection{Commissioner Analysis}

As my recruitment of commissioners was to provide additional information to help refine my CMOC regarding Woodlands' issue with referrals I was unable to use the framework used for PA, staff and service-user data. I analysed commissioner data using thematic analysis (Braun \& Clarke, 2006) (see section 2.9.4). I identified twenty-three initial codes from the commissioner dataset, and these were refined into four overarching categories (see Appendix DD and Appendix EE).

Within the findings' chapters, pseudonyms will be used throughout to protect participants' anonymity. It should be noted that I received the quantitative data anonymised and therefore, I was unable to match the qualitative data from service-users with their respective quantitative data. I have therefore used different pseudonyms within the qualitative and quantitative chapters. Demographics specific to the quantitative data can be found in section 7.1. 


\subsection{Who was Woodlands Intended to Support?}

My rationale for presenting this data first is to provide an understanding of who PAs described as the intended service-users, which will enable me to compare this with who actually accessed Woodlands (see section 4.5). This will allow me to identify any convergence and divergence between the anticipated service-user group, and the realities of who was accepted at Woodlands. This was necessary because as the project progressed a frequent complaint of participants related to the low service-user numbers at Woodlands, ultimately leading to an adjustment in who was subsequently accepted as a referral.

I show that the new resource of a recovery-oriented inpatient service was not working as intended as referrals were not secured, and there was a very real possibility that Woodlands could close. I explore any reasons proposed by the research participants for these difficulties. By better understanding the demographic of who Woodlands was intended to support, and who was referred and accepted, it was anticipated that key contextual factors would be identified that prevented Woodlands, as a newly established service, from operating as intended.

Joe and Ethel suggest that Woodlands was intended for a specific subset of service-users, as both set out in their responses:

"we knew the type of clients we were going to be working with er are the sort of clients who have been stuck" (PA Joe)

"it's going to be people who have been institutionalised for a long time" (PA Ethel)

Joe refers to the anticipated service-user group as individuals who have not progressed within the mental health system. He assigns them a specific quality of being 'stuck' to distinguish them from other assumed categories of service user. This suggests that this subset of service-users may require a different approach to their treatment compared to most other mental health service-users. Ethel proposes that these individuals will have been in institutions for a prolonged period. She uses the term 'institutionalised' to signal 
again that this is a group with a specific set of needs. It might be understood that these needs are intractable to some extent and therefore will present a challenge to the service in facilitating this recovery.

Joe further identified more specific service-user characteristics that he considered reflective of this service-user group:

"if you had somebody who is just not interested in their own recovery, at all, and we work with clients who are perhaps in their 40s, they have tried living independently, it hasn't worked for them, they like living in a (.) sort of (.) hotel environment (.) you know an independent hospital (.) I get my three meals a day, TV, a nice warm room (.) I (.) I struggle to cope (.) Woodlands isn't going to work for them, because the ambition is not to be there very long (.) so they have to be people that see that this is a stepping stone, this isn't somewhere they will live for some years" (PA Joe)

Joe appeared to contradict his own category of being 'stuck' and Ethel's category of 'institutionalised'. To support his construction of the ideal service-user, Joe created two categories to explain who he considered suitable for Woodlands. The first category positioned individuals as wanting to be cared for, and who are uninterested in their recovery or independent living. A group we might imagine are indeed 'stuck' and 'institutionalised'. Whereas, the second category reflects service-users with an ambition and desire to leave inpatient services. Joe positions the latter group as the intended service-user group for Woodlands. It is perhaps unlikely that individuals who are motivated, interested in their recovery and want to quickly move out of services are going to be 'stuck' in services. It might be that service-users who are ambitious and see their time in services as temporary are less likely to require a service like Woodlands. It is also questionable as to whether individuals with these attributes will be sent to a locked service given the prioritisation of least restrictive practice within the MHA Code of Practice (Department of Health, 2015).

Service-users who lack ambition, skill or belief to live independently within the community, who Joe appears to dismiss, may be the very individuals who require and are referred for targeted and specialist support in secure services. Joe's inconsistent construction of who Woodlands was designed to support suggests there may be ambiguity surrounding who the intended service-user group was. If PAs do not have a 
clear consensus on who Woodlands was intended to support, this could be problematic at the micro-level, as staff may lack clarity on who to accept. These data extracts may also reflect a debate among PAs about who Woodlands is designed for, and that this debate remained unresolved.

Joe's construction of categories of who is, or is not, suitable arise from his position as a PA and may be based on idealised types in the absence of real-world examples. In the following two sections I present my analysis of data from a range of participants relating to real-world examples in the form of who was referred and accepted at Woodlands, and the difficulties Woodlands faced in securing referrals.

\subsection{The Troubles of Securing Referrals}

One challenge that participants referred to related to the number of referrals Woodlands was receiving. My analysis of PA, staff, commissioner and service-user accounts showed there were differing factors that were identified as contributing to the service's difficulty in securing referrals. I will show, using my analysis, that there was a discrepancy between the intended service-user group presented by PAs and staff (see section 4.4), and the realities of who was referred and accepted at Woodlands. Within the analysis I consider the current market demands for locked mental health provisions, how Woodlands was categorised by commissioners and what the claimed consequences of being unable to secure referrals were for Woodlands.

\subsubsection{Factors Informing Commissioner Decision-Making}

Commissioner identified numerous factors that were used to inform their decisionmaking. Factors that informed commissioner decision-making related to the location of the service, least restrictive practice, evidence of the service's effectiveness and whether the Local Health Board (LHB) had an NHS-run service that could provide the treatment.

To provide some context, Local Health Boards (LHBs) are responsible for the commissioning of medical services and the provision of front-line services in Wales. There are seven LHBs in Wales, each covering a certain geographical location. I interviewed commissioners from three different LHBs, including the LHB Woodlands is located within. 


\subsection{Location}

Commissioners identified that the proximity of the service in relation to service-user's home and family support was positioned as a reason why commissioners were not referring to Woodlands:

"yeah we would try and place someone close to home erm just for family, cause obviously family support is very important but also the cost as well (.) and erm cause we need to go to a panel so that if if we are placing someone far away every time we review them it's expensive, or they question why we place someone in certain areas... so the commissioning board is very hot on our tails at the moment as well (.) so we've got to be very specific and careful about where we place our patients" (Commissioner Laura)

Laura suggests that commissioners are scrutinised for their decisions and implies that commissioners need a good rationale for making any choices regarding placements. Location and proximity to service-user's social network appeared to form part of her rationale. There appears to be a prioritisation of local services to avoid service-users being sent away to access treatment.

Laura's explanation of how location influences her decision-making was supported within a recent National Collaboration Commissioning Unit position statement (2020) which suggests that placing individuals in hospitals located in Wales was a priority. This is supported by data which shows that since $2018 / 2019$ the number of patients placed in hospitals located in Wales has increased by $4 \%$ whilst the number placed in English hospitals has decreased by $14 \%$ (National Collaborative Commissioning Unit, 2020). The same position statement also indicated that $61 \%$ of individuals were admitted to a provider less than 50 miles from their significant postcode, and a further $18 \%$ accessed their care at a provider within 50-100 miles. This appears to emphasise that the National Collaboration Commissioning Unit place location as a key determinant when deciding where individuals access their care and treatment.

Laura indexes both family support and cost, which shows that there may be competing reasons when commissioners make decisions about placements. The framework uses a 'quality then cost' approach, which the National Collaborative Commissioning Unit states provides a competitive mechanism between providers who are meeting the quality 
standard, which enables a highly competitive environment (National Collaborative Commissioning Unit, 2020).

The proposal that location is one key determinant in where service-users access treatment suggests that the potential pool of people who will be considered for Woodlands may be limited to those geographically nearby.

\subsection{Least Restrictive Practice}

The principle of least restrictive practice was also referenced by all commissioner participants as a key feature of their decision-making. Rowena suggested that there was a preference for open conditions when this was appropriate:

"I would be reluctant (.) well everyone would be reluctant that if someone has been in an open acute unit to then move them up to a rehab unit if what they needed was rehab and its locked, what we use a lot these days is open rehab because (.) so we have a couple in locked rehab, but a lot of the people we have had in locked rehab have been the female self-harmers who need those slow steps out (.) sorry that was bad saying female self-harmers females who self-harm (.) but what we have found even with those young ladies is they tend to move to the open rehab so they still get the nursing care, but it's actually what they will do when there is an open door that needs to be tested. So actually, just putting them in another locked environment doesn't necessarily help them develop in the way in the way that we need to see, we need to see how are they going to manage when they go out and access the community" (Commissioner Rowena)

Rowena's account suggests that achieving and maintaining open-door conditions within a service-user's treatment pathway provided commissioners with an opportunity to assess individual's ability to manage in the community. Least restrictive practice is expected to be considered by those detaining individuals under the MHA, therefore Rowena's reference to this established principle within her role as a commissioner is unsurprising. The literature shows that there has been a focus upon using alternatives to traditional locked wards through the introduction of open-door policies (Huber et al., 2016) following the process of deinstitutionalisation (Bachrach, 1997). This aligns with Rowena's preference for least restrictive and community-based options. Despite this policy shift to least restrictive options, Woodlands operates with locked conditions, which, by nature, may challenge central ideas of recovery, such as choice and independence. 
Rowena associated locked conditions with self-harm, proposing that individuals who pose a risk to themselves may be considered for locked services. The justification used for treating individuals within locked conditions has been to avert harm to themselves and/or others (Salize \& Dressing, 2004), which supports Rowena's understanding of who needs locked services.

A recent position statement from the National Collaborative Commissioning Unit (2020) showed that over the past 7 years the use of medium and low secure services has decreased by $38 \%$ and $8 \%$ respectively, which supports Rowena's preference for using least restrictive practice. Whereas, the data shows that locked rehabilitation has increased by $39 \%$ and services with open conditions has decreased by $42 \%$ (National Collaborative Commissioning Unit, 2020), which shows a preference towards the use of locked rehabilitation. This is of interest as Woodlands is classified as a locked rehabilitation service on the framework but has had difficulties in securing referrals.

The notion that least restrictive practice is a factor in where service-users are placed suggests that the potential pool of individuals considered for Woodlands may be limited to those who pose a risk to themselves, and where community-based, or open conditions, may not be appropriate.

\subsection{Is there an NHS Service to Provide the Care?}

The final factor identified as informing commissioner decision-making related to whether the LHB had its own NHS service that could provide treatment before referring to a private facility:

"we are essentially using the facility predominately for females, because we have male locked rehab within the health board and it will always be using that in the first instance unless there is a specific reason why a male would need to receive locked rehab outside of our own services (.) this would be the only reason we would use it" (Commissioner Roy)

Roy's account indicates that commissioners prioritise NHS-provided services before outsourcing to private facilities, such as Woodlands. Roy works for the LHB for the area within which Woodlands sits, therefore his account highlights that there may not be a 
great need for a service providing locked rehabilitative support for males. The lack of need for this type of service for males further limits the pool of individuals that Woodlands can focus upon as potential service-users.

It is important to note that Roy classified the service as a locked rehabilitation, whereas PAs classified Woodlands as a Recovery Centre. These different classifications may have resulted in divergent conceptualisations of what Woodlands provides and who was suitable for referral, which could have also contributed to a discrepancy between who PAs intended for Woodlands to support and who was referred in practice.

One PA noted that the classification of Woodlands on the national framework, which is used by commissioners to identify suitable placements for service-users, did not capture what the service was providing:

"there are only 12 lots which are you know secure (.) medium secure (.) locked (.) unlocked (.) where is the one that describes what we do (.) we are not (.) we are pigeon holed into something that we are far better than (.) but there doesn't appear to be an appetite from the framework to be dealing with this erm in a better way" (PA Joe)

Joe suggests that there are pre-defined categories which are used to classify what a service provides and is then used to find a suitable placement for service-users. He suggests that the framework does not have the flexibility to acknowledge when a service is providing something outside of these parameters. Joe's account suggests that Woodlands is therefore inappropriately categorised on the framework and therefore what it provides is not captured. This suggests that there may be a lack of flexibility within the wider referrals system to acknowledge alternative forms of service delivery. This highlights a discrepancy between how commissioners and PAs categorised Woodlands, which may have contributed to some of the challenges the service encountered, such as insufficient referral numbers and needing to adapt its service-user criterion to secure referrals (see section 4.5.1.3 and 4.5.2).

\subsection{Evidence of Effectiveness}

Once the three factors above had been determined, commissioners considered what evidence was available regarding the effectiveness of the service in supporting recovery. 
I show, using my analysis of commissioner and PA data, that there was a discrepancy between what both parties understood evidence of effectiveness to be.

Commissioners wanted evidence of service-user progress; as Woodlands was a new entity it was difficult for the service to provide evidence of progress, so they depended upon advertising their awards and testimonials as a proxy for evidence. However, this evidence does not appear to be enough for commissioners to use when deciding upon service-user placements. I show, using my analysis, that being a well-meaning charity with a new service was not enough to enter the market in the way they hoped or expected.

Roy suggested that Woodlands and the organisation were not as well-established compared to other providers:

"one of the issues is they [other services], they are generally more established so there systems are more tested, generally they are part of a much wider company so for example Priory for example, Priory have hospitals and they will have low secure locked rehab open rehab addiction facilities and so on and so forth, they have the infrastructure there, the systems in place, the care planning expertise that erm (.) so from my understanding with Woodlands it's under the [organisation name] umbrella I don't know whether experience would have come from within cause they are in some respects starting out quite new (.) which is sometimes a good thing cause they don't feel they have to follow the same rules and routines that everybody else has, but you could counter that with them not having that slick operation that erm (.) I don't know advertising what they, they don't have the evidence, we have had three hundred patients through 80 percent have moved onto you know they may not have that either (.) I understand and in some respects I am very sympathetic to how difficult it must be for them as well" (Commissioner Roy)

Roy highlights that other services can provide evidence of service-user progress, which Woodlands could not provide. Roy's account alludes to Woodlands not having a track record of providing inpatient care and therefore it remained an unknown quantity. Roy appears to question the experience of those at Woodlands, and perhaps needs additional information to reassure him that the individuals involved have the necessary expertise. It has been reported that in the absence of relevant information to guide choices relating to healthcare providers, decisions are dependent upon reputation (Dijs-Elsinga et al., 2010). Reputation is a perceptual representation of an organisation's past actions and prospects, which creates the service's overall appeal when compared to rivals (Roberts \& Dowling, 2002). Roy's account alludes to Woodlands lacking a reputation of providing inpatient 
care, which positions them as worse off compared to other service providers with more established reputations.

Roy's perception of Woodlands contrasted with PA accounts which showed they held the belief that Woodlands provided service-users the best opportunity for recovery:

"I'm just shocked (.) and erm saddened that its allowed to happen (.) because surely its people's lives we are dealing with here and if we have a service that can offer a person the best chance of (.) treatment (.) and hope, and recovery, then surely, that's something that needs to be at the forefront of any commissioner's mind (.) but then maybe I'm a bit naive in that respect, but that's the way I think about it anyway" (PA Ethel)

"people should be knocking down our doors to be getting people referred (.) we know they've won an award (.) you know it's a fantastic environment for people (.) its therapeutic (.) they are getting the best treatments (.) so from a clinical perspective you would expect people to be coming and knocking down our doors saying we have people we want to come there (.) and that is a huge frustration" (PA Ethel)

Ethel's accounts contrast with commissioners' who suggested that Woodlands was less established compared to other providers, whereas Ethel considers it the best. Ethel's account appears to be an act of faith in Woodlands, she strongly believes and values the service's claimed focus, even in the absence of evidence of service-user progress. Her account suggests that having a recovery vision will be enough, but my analysis of how decisions are made in the current market shows that just having a recovery vision is not enough.

Ethel uses an award won by Woodlands as a signifier of their quality in place of actual evidence of recovery. The award Ethel is referring to was for recognition of outstanding initiatives and innovative efforts and was awarded prior to Woodlands accepting any referrals. The use of the award appears to be an attempt to highlight the quality of the environment, but perhaps omits what it is commissioners want to see and hear before referring an individual to the service. The award was positioned as a selling point of the service which could be an attempt by Ethel to strategically build a strong competitive position against its comparators. There may have been an over-reliance upon the award Woodlands received as PAs were unable to provide the evidence commissioners wanted or needed. Ethel's account highlights a discrepancy between what PA and commissioners 
consider as evidence of service quality. PAs based quality upon the service's values, their belief in Woodlands and its potentiality, as well as securing an award from an external body; whereas commissioners wanted evidence grounded in quantifiable data relating to recovery progress.

Ethel assumed people, presumably commissioners, would automatically refer serviceusers to Woodlands based upon its claimed credentials and facilities. Ethel's accounts imply that the service's existence alone would attract referrals, which overlooks the reality that a reputation needs to be developed, which requires ongoing, organisational commitment (Gibson et al., 2006). This suggests there may have been some naivety in the business approach of PAs, as they assumed that establishing a new service would naturally result in service-users being referred.

Reputation has been identified as a factor that can influence which providers are selected to provide service-users care (Akıncl et al., 2005). Reputation therefore is not an autogenous concept; it is developed through complex exchanges and interrelationships between stakeholders that occur overtime (Mahon, 2002) and cannot be instantly achieved as assumed by Ethel. This may suggest that there had been limited engagement between Woodlands and commissioners to develop a reputation as a credible provider, or the fact that the service was new, meant there was insufficient time to develop this reputation.

Ethel appears over reliant upon the belief that Woodlands was unique, which would transcend market needs and the factors used to inform commissioner decision-making. Ethel's accounts fail to consider other factors that may influence where individuals are placed, such as location and price. This may suggest that there was a limited understanding of the market in which they launched their new service, and how decisions are made regarding where individuals access treatment.

Ethel positions Woodlands as passive through suggesting that the situation was 'allowed to happen', alluding to having no control or power to address or improve referrals. Ethel locates responsibility with commissioners and questions how commissioners make decisions about service-user placements. Locating responsibility with commissioners 
creates an opportunity for Ethel to externalise blame and distance the PAs from responsibility.

It appears that PAs see their model of care as distinctive, and distinctive enough in their view to warrant commissioners taking a chance on placing individuals at Woodlands. Commissioners appear wary of this and appear to prefer tried and tested rubrics when making decisions on what category of person is referred to specific categories of service. This highlights that there is a tension between Woodlands attempt to provide an alternative model of care, and commissioners wanting to use approaches that are evidenced and familiar.

I have shown using my analysis that there are key factors that informed commissioners' decision-making. These factors therefore limited the pool of potential individuals Woodlands could support. Woodlands was limited to receiving referrals from certain geographical parameters. The pool of potential service-users was further limited by a prioritisation of whether the LHB had an NHS service. The LHB that Woodlands is affiliated with has a locked male rehabilitation facility, Woodlands may receive a limited number of male referrals. Although the LHB may have a male service, some males were referred and accepted at Woodlands, however, I am not aware which LHB was responsible for these referrals.

When the factors are considered collectively Woodlands is restricted to referrals from individuals within close proximity, who are female and require locked conditions. This represents a small subset of service-users which makes the trouble in securing referrals unsurprising. As Woodlands was new, PAs did not have evidence of effectiveness and quality available and therefore relied upon the claimed focus of the service and accolades it received. However, my analysis shows that from commissioners' perspectives having a recovery philosophy and providing a new service was not enough to gain advantage in a commercial and competitive arena in the way anticipated.

\subsubsection{Current Market Demand for Locked Services}

Commissioners categorised the service as a locked rehabilitation facility, which could have contributed to the discrepancy seen between who was referred and who PAs designed 
Woodlands to support. PAs saw the service as designed for people interested and motivated in their recovery and who had psychosis-type diagnoses. Commissioners, however, proposed that individuals with a personality disorder diagnosis (PD), challenging behaviours and who posed a risk are the individuals likely to be referred to Woodlands. I argue, that in the current market, it was highly unlikely that Woodlands would receive the people that PAs anticipated.

Roy alluded to individuals with a PD forming the subset of service-users who he considers suitable for Woodlands:

Researcher: is there a demand for that service and could that be a reason why the referrals haven't been going there

Commissioner Roy: erm (.) well there definitely is (.) there is a consistent demand for female locked rehab (.) I mean (.) I (.) I (.) partly understand that is possibly a politic issue (.) so many females are determined as needing a locked environment erm (.) but I guess that's not the nature of this interview but it is quite noticeable and it is, it tends to be your particular type of client group (.) and I don't like to categorise people but its people with emotionally unstable personality disorder, self-harm, erm, and present with challenging behaviour (.) that we as a whole, and I don't just mean our health board I mean er, society wants us to address (.) I do feel if, if it were that the preference is to deal with (.) for the service to deal with things around psychosis or severe mood disorders or whatever they will continue to not have many referrals in reality (.) I don't think it's sad, I think it goes back to, what are the fundamental issues in society that we need to deal with?"

Roy suggests that there was a consistent demand for locked rehabilitation and uses his experience of referring individuals to these types of services to determine who he expected to be suitable. Roy identified individuals with a PD, and/or challenging behaviours, such as self-harm, as the individuals likely to be referred to Woodlands. What is of interest is what Roy is saying is in direct contrast to the position of Woodlands proposed by PAs. He suggests that if the service is a locked rehabilitation then this is a needed service for women with a PD. If the service is something else, such has a locked recovery service for individuals with psychosis, then this is not needed in the current market.

Roy appears to be rejecting the positioning of Woodlands on the basis that there is no market for a recovery service of the type that PAs proposed. Roy's account works to claim 
wider authority for his position in that it is what society wants or needs, so his rejection of the service and its model is not personal, but one that is mandated based on his assessment of societal demands. The wider power of society appears to provide legitimacy for his decision-making, as there was an imputed responsibility to 'deal with' the fundamental issues of society. Roy concludes that Woodlands will continue to have difficulties with referrals if it does not adapt and accept individuals with a PD. In addition to $P D$, individuals who pose a risk to others were likely to be referred to Woodlands:

"there was another lady we referred but they declined her because of her violent behaviour (.) so you know that is quite fine, but we have now referred her to another locked and open rehab and they have both accepted her, so I think you know we would never expect a provider to take a person that erm, would put their staff at risk and whose needs they couldn't meet (.) but that would suggest to me that maybe other locked rehab units have a higher tolerance of some behaviours than this one (.) I mean that is just my assumption she hasn't gone yet but she has been accepted and this placement turned her down, so that would suggest that there is some tolerance there which doesn't marry up with the locked bit you know" (Commissioner Rowena)

Rowena alludes to a discrepancy between Woodland's categorisation of locked rehabilitation and their acceptance of service-users with risk behaviours. This highlights that commissioners understanding of what behaviours can be managed within locked rehabilitation and what Woodlands are willing, or able to manage, do not align. Rowena assumed that people with risky behaviours would be an appropriate referral, which appears reasonable as many individuals in a locked service will have been sectioned and deemed a risk to themselves or others. Rowena uses an example of an unsuccessful referral to highlight that Woodlands declined service-users because of their risk. This suggests that risk was used as a marker of suitability at Woodlands. The alleged refusal of individuals who pose a risk appears to overlook the reality that service-users detained under the MHA will have some form of risk to be sectioned.

Rowena expresses some uncertainty about what Woodlands is meant to do, and who it is meant to support, which may arise from the mismatch between PAs claims of promoting recovery and its design as a locked service. The decision to provide a locked service appears to conflict with the intended plan proposed by the PAs to support individuals who are motivated and recovering. This highlights ambivalence between the restrictions 
imposed at Woodlands and the individuals the service was designed to support, which appears to have been picked up by external agencies, such as commissioners.

There is a juxtaposition between who the service was intended to support and the decision to have locked conditions as part of Woodlands design. The current demand for locked services was for females with a PD, and those who pose a risk to themselves or others. This suggests that there may be a need for Woodlands to reconsider who the service is intended to support and consider how it can adapt its approach to ensure it meets the needs of all individuals accepted at the service.

\subsubsection{Reacting to Meet Market Demand}

Woodlands did eventually deviate from its service-user criterion, meaning individuals with a primary diagnosis of PD were eventually accepted. This highlights that Woodlands was flexible in who they accepted and adapted to the market demands; however, this adaption did present some challenges within practice.

Walter, a staff member, did not know how to support some of the individuals who were eventually accepted at Woodlands:

"one particular guest just wants to get a flat so she can have a drink and have her friends round and take drugs and not bother anybody, so she's not really interested in improving her situation, that's really her only ambition, so you are limited then in what you can do with that person then in a sense" (Staff Walter)

Walter's description suggests there was a lack of therapeutic options when supporting persons who wanted to adopt an unorthodox lifestyle, which may not improve their chances of recovery or could potentially cause harm, such as taking substances. This suggests that the support service-users received from staff may be dependent upon what their ambitions were and whether staff felt able to support these choices.

Walter's account appears to locate responsibility for behaviour change upon the serviceuser and omits the role he, and other staff, may need to play in ensuring they are ready and supported (Prochaska \& DiClemente, 1982). However, proposing that service-users need to change suggests that they can be fixed, and that the solution rests with the 
individual, rather than as a collective at societal level (Deegan, 1988; Recovery in the Bin, 2019).

As shown in section 4.5.1.2, there was a consensus amongst commissioners that individuals with a PD are more likely to be referred to Woodlands. Walter proposed that the approach to care needed revising to meet the needs of individuals with a PD:

"if we are having people like this who are more personality disorder patients then how can we adapt our service to benefit them better because (.) I don't always feel we know exactly what we are doing with them (.) and treatment for personality disorder is very different to other illnesses and I am not sure the clinical staff are particularly well trained for that to be fair, I don't think they are necessarily that well equipped to deal with it"

(Staff Walter)

Walter created a category of 'people like this' and then populated this category as individuals with a PD. Walter then highlights a problem with staff competence and recognises the need for variance in treatment approaches. He suggests that individuals with a PD require different care compared to individuals with other illnesses. Walter suggests that Woodlands had not adapted to meet the needs of these service-users. If the service intends to accept these referrals, then a significant shift in focus would be required to ensure their needs are met. Walter's account suggests that there is a need for reorientation so that Woodlands and its staff are equipped to support individuals with these diagnoses.

Walter went further and suggested that he was unable to help or support individuals with a PD in their recovery:

"we are working with personality disorder here there is nothing we can do we can't help these people recover" (Staff Walter)

Walter appears to lack therapeutic optimism and is helpless when he discusses working with individuals with PD. Walter's account appears to align with the discourse surrounding individuals with a PD which is largely negative and pessimistic (Bowers et al., 2006). Given Walter's account, he may not have had any specialist training to work within individuals with PD, which may explain his lack of competency when working with these individuals. Walter's account suggests his view of recovery is grounded in a clinical perspective, where 
symptoms are reduced or cured (Slade et al., 2014), as opposed to viewing recovery through the lens of the service-user living a satisfying and hopeful life (Anthony, 1993). Literature has suggested that some clinicians do not feel that recovery is an approach relevant for individuals who do not have a psychosis diagnosis (Slade et al., 2014). This is despite emerging evidence which suggests that recovery is relevant to non-psychosis clinical populations, including diagnoses such as borderline PD (Katsakou et al., 2012). Laura proposed that Woodland's approach was not suitable for individuals with PD:

"where it broke down with my patient was erm (.) she needs boundaries and consequences and I am aware that Woodlands is kind of patient (.) sort of (.) what's the word I'm looking for (.) you know it's up to the patient (.) I'm lost today my minds gone (.) erm (.) maybe gives too much responsibility to the patient at the start, so she never drinks but she went out and had a drink which is out of character and then went AWOL [absent without leave] but then was allowed out a couple of days later, whereas that doesn't work for my type of patient cause she needs you know, consequences to her actions and she was diagnosed with a personality disorder erm, if you give for example that patient an inch she will take a mile every time so it was just spiralling then" (Commissioner Laura)

Laura suggests that individuals with a PD require a different approach to the one offered at Woodlands, such as needing boundaries. This supports Walter's account which suggested that individuals with a PD require different treatment approaches. Similarly, to Walter, Laura suggests that Woodlands was not meeting the needs of individuals with PD. Laura's account recognises that a one-size-fits-all approach to service delivery for different service-user groups was not appropriate. For example, the needs of individuals with PD are not the same as those of someone with a chronic psychosis. Whilst the philosophy can be similar, the way this is actioned needs to differ, as does the skillset of staff, which Walter alluded to.

Woodlands did adapt its criterion and accept individuals who it was not originally designed to support. This resulted in some staff being unsure of how to support these individuals, which is unsurprising given that Woodlands was not designed for this subset of service-users. This evidences that staff may not have had the flexibility, skillset or training to adjust Woodlands' approach to meet the overall market demand of locked services, and the needs of the individuals. This may require staff to be upskilled so they can competently and confidently support individuals regardless of their diagnosis. 


\subsubsection{Consequences of Not Securing Enough Referrals}

Woodlands is a 16-bed service, and throughout the study period the service-user occupancy ranged between 4-6 individuals, meaning the service was running at less than half of its full capacity. Participants identified several consequences of operating at these levels of occupancy. Whilst I am not presenting these accounts as hard evidence of cause and effect, I will rely upon what individuals claimed were some of the impacts of Woodlands not securing enough referrals.

\subsubsection{Staff Morale}

The occupancy at Woodlands was lower than both PAs and staff anticipated, and this was presented by participants as causing low staff morale:

"biggest part of the problem is we are not busy enough at the moment (.) cause I think if we are busier than the staff would have more motivation as well (.) I think it's a struggle where you have only 3 or 4 guests because, because you are almost one to one" (PA Joe)

"well people [staff] have come and gone quite a bit and I think that's because there is a lack of activity you know (.) it could be a bit demoralising to some extent (.)you want to feel as if you are doing something worthwhile, that's why you do jobs like this you know"

(Staff Walter)

Joe proposed that the motivation of the workforce was associated with service-user numbers. Walter took this further and proposed that the low occupancy was the reason for the high staff turnover. Walter proposes that the context in which he performed his job lacked activity, which contributed to reduced motivation; alluding to there not being enough activity to motivate staff. Herzberg et al's (1959) two-factor theory of motivation, identifies motivators as factors that are intrinsic to the job, such as achievement, growth opportunities or increased responsibilities and these factors encourage staff to work harder. Herzberg et al (1959) labelled factors that caused staff dissatisfaction as hygiene factors. These factors are part of the context of which the job is performed, as opposed to the job itself. Using Herzberg et al's (1959) theory to understand Walter's account it highlights that the lack of activity was a hygiene factor that negatively influenced staff motivation and retention. 
These accounts could also be read as exposing the staff as not having the range of skills or expertise to use their time proactively and positively to support service-users in their recovery. Walter's account is interesting as mental health staff often complain of there not being enough staff or time to do everything required of them (Coffey et al., 2019a), however in a context where both are available, this also did not appear to work.

\subsubsection{Service-user Responses}

Service-users' expressed mixed responses to the low occupancy at Woodlands:

"to be honest it [low occupancy] has been a bit frustrating, I should have just left and gone home straight away, cause now I feel more lonely here than I did before (.) and I only came here cause I didn't want to be on my own in the house so yeah if anything, it has made me realise I am on my own" (Service-user Ellen)

"I like it to be a little bit more lower cause I am a bit wary of loads of people" (Serviceuser Diana)

For Ellen, the company she expected to receive at Woodlands did not meet her expectations, which became a source of frustration. The lack of social connections available made Ellen question her decision to go to Woodlands and she concluded that she would rather be on her own at home, than alone at the service. Ellen's account contrasts with PAs who alluded to service-users having the undivided attention of staff, (see section 5.4); whereas Ellen expressed loneliness and isolation. The experience of loneliness at Woodlands will also be discussed as a potential trend in the quantitative results (see section 7.8.1.5). Whereas, for Diana, the low occupancy suited her because of her concerns of larger numbers of people. Ellen and Diana's accounts highlight that there was variance in service-user experiences of occupancy at Woodlands.

Kelly, a staff member, constructed the problem of low occupancy as staff being continuously approached by service-users for help, which she associated with dependency:

"a few of the guests have been like, oh I'm bored, there is no one here, when I was at [another service], I had this person to talk too, blah blah blah, so I can see their frustration with that (.) and they tend to lean on us a lot more (.) so it's like, I'm bored, I'm bored, and it's like well you have to be able to do something to occupy yourself (.) so 
then (.) they are coming to you, can I do this? can I do that? and its constant (.) and you don't want to be constantly doing things for them, and filling in their time, cause it's not going to be like that (.) it really isn't going to be like that (.) but it must be hard for them" (Staff Kelly)

Kelly does not appear to view service-users approaching staff for help as an opportunity to support these individuals to develop skills, such as how to use their time. Kelly's account positions service-users as solely responsible for managing their own time and overlooks that they may require support to do this. This could be viewed as a hands-off approach to recovery, which has been seen elsewhere within ROC literature (Aston \& Coffey, 2012).

\subsubsection{The Longevity of Woodlands}

Ethel accounted for how securing and accepting more service-users would, unsurprisingly, place the service in a better financial position:

"if we don't have the referrals it doesn't become a viable option (.) so we have to (.) we have to look at all of these things, cause it has to be a viable option, it has to be selfsustaining and at the moment the barriers for us are the referrals" (PA Ethel)

Ethel expressed that Woodlands was dependent upon securing placements to generate a financial income as it is a business that needs to pay for itself. Ethel's account highlights that there were costs attached to running a service and Woodlands needs to make money to survive, in the same way as any other business. This is even more relevant as a not-forprofit charity delivers Woodlands and therefore sizeable reserves are not available to keep the service operating. This shows that despite the well-meaning intentions of PAs in attempting to provide something different in the current mental health market, if this alternative service is not considered and used by commissioners, then Woodlands will be unable to continue operating. This highlights the challenge Woodlands faced when entering a competitive, commercial arena which appeared to favour tried and tested forms of service delivery. 


\subsection{Summary}

In this chapter, I have shown, via my analysis of accounts from various participant groups, that Woodlands had trouble securing referrals. There was a discrepancy between PAs accounts and the realities of who was being referred to locked services and the factors informing commissioner's decision-making. This highlights the importance of services, particularly if new, understanding the demands for mental health provision to ensure that they are addressing a gap in the market, or that there is a need for that type of service. My analysis suggests that there may not be a great need for a locked service promoting recovery, as individuals are likely to receive this type of support within open conditions or the community. Commissioner accounts alluded to there being an issue with the service promoting recovery but restricting the liberty of service-users through operating with locked conditions. This ambiguity appears to have played out in Woodlands having trouble in securing referrals. The arguments presented cast doubt upon whether there is a need for a private-run, locked service that is intended to support individuals who do not require much help in their recovery. This highlights that the service's initial approach did not align with the market demands of who required locked services. The issue of low occupancy is therefore likely to persist if the service-user criterion and Woodlands' approach are not adapted to meet the needs of all individuals who are accepted.

My analysis also highlights the challenges that Woodlands encountered with being a new service attempting to provide an alternative model of care within the wider mental health system. Commissioners classified the service as a locked rehabilitation facility, and therefore Woodlands was compared to other locked rehabilitation services. PA accounts suggest that they believe Woodlands offers something different to these services, however this was not reflected in how commissioners categorised the service. This suggests that the way in which services are categorised within the referrals process may not have the flexibility to recognise services that do not fit into the pre-defined categories of service delivery. This was evidenced by commissioners referring individuals with a PD and who posed a risk, to Woodlands, as these are the category of individuals currently requiring locked rehabilitation. 
When Woodlands did not accept these individuals or did not provide the type of care commissioners expected, such as using boundaries and giving less independence, commissioners expressed there was a discrepancy between the classification of the service as a locked rehabilitation and who they were able to cater for. This highlights the challenge Woodlands faced when attempting to provide an alternative form of treatment whilst being categorised as a type of service that they never intended to deliver. This places Woodlands at a disadvantage as they are being held to the standards and expectations of a locked rehabilitation, despite this not being the service they aimed to or wanted to provide.

Another challenge Woodlands encountered with being a new service was it was not able to provide commissioners with the evidence they required which showed service-user progress, resulting in commissioners defaulting to tried and tested service providers. Due to this, Woodlands was in a paradoxical situation of needing to provide quantifiable evidence of progress but not receiving referrals in order to build up this evidence base. This highlights that Woodlands encountered difficulties with being a new service trying to establish themselves within a commercial environment, which was enhanced by attempting to deliver a new and alternative form of service delivery. The key findings in this chapter have been used to inform the CMOC presented below (see Figure 7). 


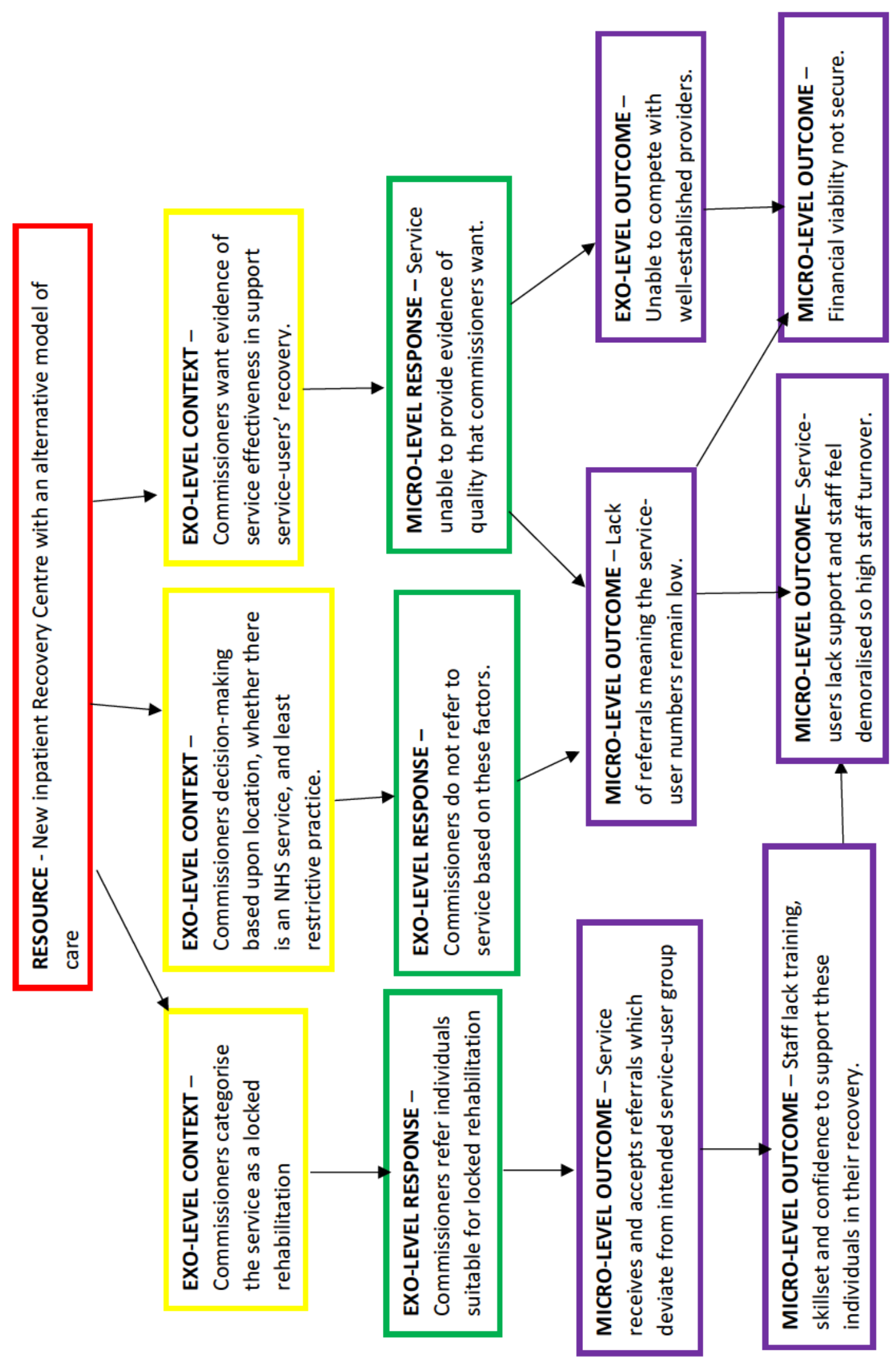

Figure 7 - CMOC for Referral Difficulties 


\subsubsection{Explanatory Power}

In sum, the following partial, fallible theory suggests that:

- Where inpatient services, and what they offer, are not congruent with commissioners' current requirements/expectations of inpatient settings, then they will struggle to establish themselves as a new provider and secure referrals;

- When services and commissioners are governed by policies that promote least restrictive practice and reserve inpatient care for those most in need, then inpatient services and its staff need to be prepared to adapt themselves to deal with the complexities of the service-user group likely to be referred to such facilities;

- When staff do not feel they have the confidence, skillset or training to support the service-user group accepted at the service, they do not have the necessary resources or knowledge to complete the task of supporting individuals in their recovery, nor can the implement the service's value of recovery.

These findings, therefore, emphasise the importance of services being designed and delivered to meet the demands and expectations of commissioners who decide where individuals access services. These individuals are guided by current policies; therefore, it is important that new mental health services are congruent with the current policy direction which is to provide care in the community where possible, that inpatient services are for those most at risk, reduction in outsourcing, and that all services promote this conceptualisation of recovery.

A key explanatory feature of the challenges Woodlands encountered when establishing itself as a provider is congruence between the service and the wider referrals system. Organisations can only succeed if their strategy is influenced by its inputs (e.g. policies, commissioner decision-making, neoliberalism) and when the work, the people who do the work, the organisational structure and the culture all 'fit' together with the inputs (Nadler \& Tushman, 1980) (see Figure 8). If they do not, as seen in Figure 7, such as staff not feeling confident to support new service-user group, being unable to provide what is 
required of them to commissioners and its culture of supporting motivated and ready service-users not aligning with policy, then services will need to adapt their strategy to be more congruent with these inputs, or their outputs will be negative. This emphasises that Woodlands original strategy may not have considered the current market demand, other service provisions available within Wales and UK and welsh mental health policies, which would have shown that Woodlands culture was not considered to be as unique as PAs proposed. These are reflected within some example 'if then' statements that were generated to understand the challenges Woodlands faced as a service (see Appendix JJ), and to support the identification of an appropriate substantial theory in the form of the congruence model (Nadler \& Tushman, 1980).

The findings from this chapter focus upon inputs in the form of commissioners and the referrals system and Woodlands original strategy to understand the environmental pressures the service encountered, which resulted in the service needing to adapt its service-user criterion for admission. 


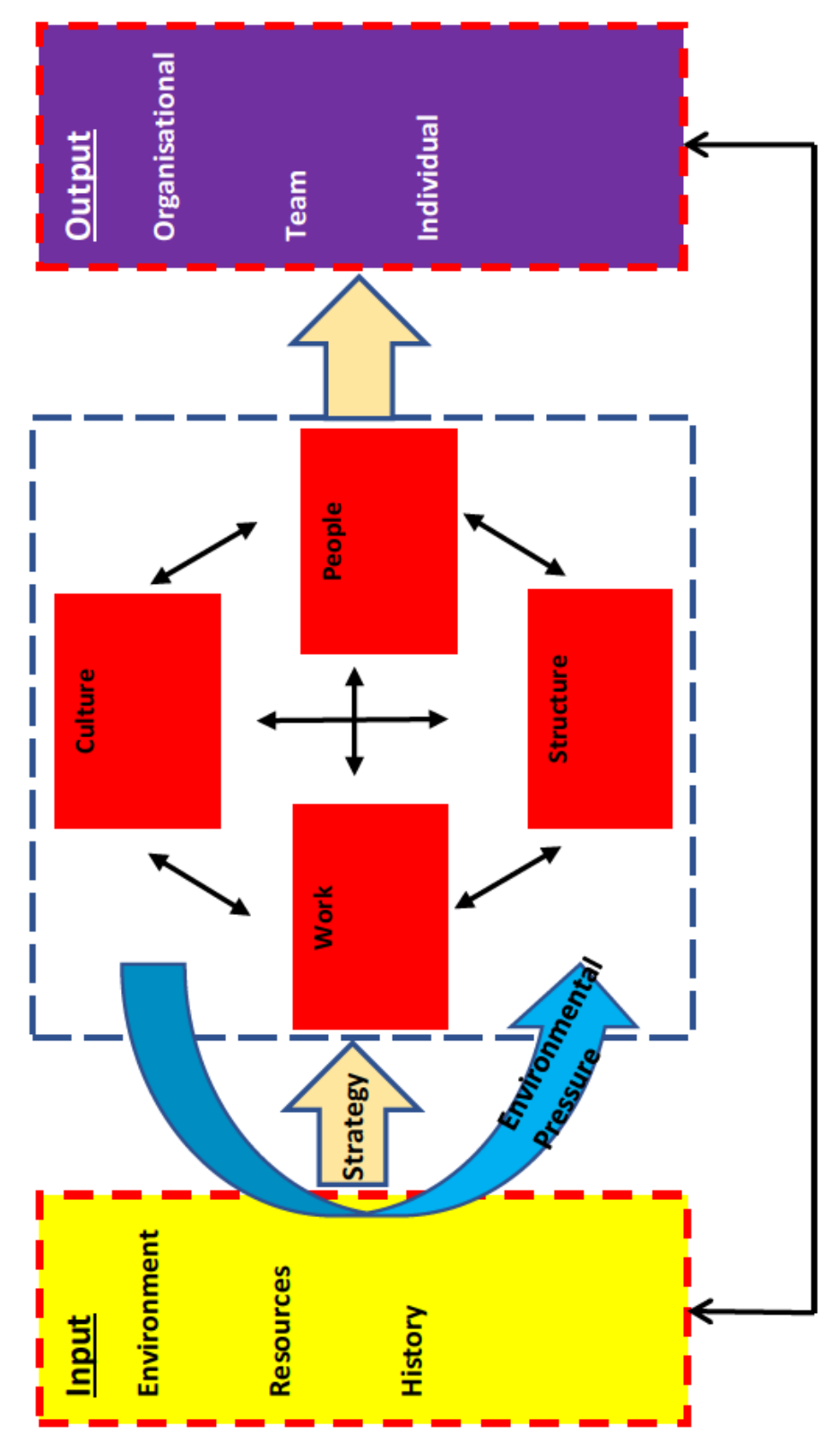

Figure 8 - Congruence Model (Nadler \& Tushman, 1980)

In the subsequent chapters I present further analysis where I examine what was said by participants about the staffing model, choice and responsibility, and finally routinely collected service-level data. In the next chapter I present my analysis of participant accounts in which they claim the staffing model was a key mechanism of Woodlands' service delivery. This chapter is presented next as I am moving my analysis from the exosystem of the referrals system to the micro-level of service operation. 


\section{FINDINGS - THE STAFFING MOdEL: THERAPEUTIC ENGAGEMENT}




\subsection{Introduction}

In this chapter, I will show, via my analysis of PAs accounts, how staff were positioned as an important mechanism in the delivery of care at Woodlands. Three different components of the staffing group were discussed by PAs, these were staff characteristics, staffing levels and peer mentors (PMs). The outcomes of interest related to whether staff were able to develop therapeutic relationships with service-users, whether service-users had access to staff when needed and whether peer mentors were able to provide a different way of working/support compared to professionally trained staff. I will start with the more general factors of the staffing group, before moving to a specific job role at Woodlands. This chapter relates to the microsystem, it will consider service-user's relationships and interactions with the staff in their immediate environment and will consider how these supported their recovery. 


\subsection{The Intended Staff Qualities}

Staffs' values and traits were positioned as important when determining their suitability for employment:

"we're having discussions about staffing and the types of staffing (.) and for me it has to be the people skills, they have to have those erm that empathic warm approachable (.) it couldn't be the I know best I'm the clinician I know what's best for you (.) that wouldn't work" (PA Ethel)

Ethel presents a selection of character qualities and skills that she considers ideal for staff at Woodlands. Ethel identifies that staff attitudes supportive of paternalistic care would not work. Paternalism implies that staff know what is best for service-users (Fritzsche et al., 2014) and positions them as subordinate and passive (Henderson et al., 2014; Knaak et al., 2017). Ethel's account dismisses the inclusion of paternalistic attitudes, which highlights her aspirations for Woodlands to provide something different in the therapeutic relationship between staff and service-users. Despite these aspirations, Ethel appears to overlook how an equal, powerless dynamic will develop within a locked service where individuals are detained under the MHA. Ethel's account, although aspirational, arguably presents an idealistic philosophy of staffing, which omits the complexities of achieving this in inpatient care.

Staff's ability to resist adopting a paternalistic approach was a cause of concern for Sandra, who acknowledged challenges to achieving a paternalistic-free service:

"it's much easier to do it to them than to have them engage with you in finding out and challenging your own assertions (.) it's so much easier to say nurse or doctor knows best than well what you do think is best for you (.) I've been always worried that it would be very easy for staff when they feel demoralised and devalued to slip into why don't we run it the usual way" (PA Sandra)

Sandra's account constructs two ends of the continuum between paternalism and personal autonomy that might arise from partnership working. She acknowledges that service delivery cannot be divided neatly into one category, such as delivering paternalistic care, or not, and that situations may arise where staff revert to more 
traditional ways of working. An illness-focused model of care was recognised as the default position for staff who were ambivalent about their practice (Hungerford \& Fox, 2014). Sandra recognises the potential of this occurring but did not propose how staff could be supported to prevent this. If the default for staff is to deal with illness-based issues that can be ameliorated, long-term recovery work may remain an elusive ideal. Sandra identified that how staff feel, such as being 'demoralised', could influence the way they engage and work with service-users, this suggests that the approach applied at Woodlands may vary day to day.

Staff were expected to move beyond focusing upon someone's mental illness to view the individual:

"the holistic approach that we have at [organisation name] recognises that individuality, and that whole personality, they see the whole person, as holistic suggests (.) and they see them with all their different interests and abilities, and their challenges (.) they don't see a set of symptoms, they see the whole person" (PA Sandra)

Sandra appeared to suggest that as the wider organisation operated in a holistic way, the natural progression would be that Woodlands would operate in this way. Other than recruiting staff with certain qualities Sandra did not provide the means for how holistic working would be achieved by staff.

PA accounts suggest that a selection of key staff qualities and how they work with serviceusers had been identified, however, less attention had been given to how these were going to be operationalised at Woodlands. The qualities and model of care identified aligns with some literature which suggests that ROC requires staff to separate the person from their illness, and that the inclusion of personhood is the foundation of all recoverysupportive relationships (Schwartz et al., 2013). It therefore appears that PAs articulation of who would be a suitable workforce aligns with those described within the literature. I have used the findings presented in this section to inform the development of an initial $\mathrm{CMOC}$ relating to how certain staffing qualities were intended to work at Woodlands (see Figure 9). 


\begin{tabular}{|c|c|c|c|}
\hline $\begin{array}{l}\text { Staff who do } \\
\text { not hold } \\
\text { paternalistic } \\
\text { attitudes } \\
\text { and have } \\
\text { people skills } \\
\text { (RESOURCE) }\end{array}$ & $\begin{array}{l}\text { The wider } \\
\text { organisation } \\
\text { already } \\
\text { delivers care } \\
\text { focused on } \\
\text { the person } \\
\text { (CONTEXT) }\end{array}$ & $\begin{array}{c}\text { Staff see } \\
\text { individuality, } \\
\text { skills, } \\
\text { abilities and } \\
\text { challenges, } \\
\text { not just the } \\
\text { mental } \\
\text { illness } \\
\text { (RESPONSE) }\end{array}$ & $\begin{array}{c}\text { Staff and } \\
\text { service-users } \\
\text { co-produce a } \\
\text { version of } \\
\text { what is best } \\
\text { for the } \\
\text { individual } \\
\text { (OUTCOME) }\end{array}$ \\
\hline
\end{tabular}

Figure 9 - Initial CMOC for Staff Qualities

In the next section, I present my analysis of service-user data in which they present their account of what staffing qualities were valuable for them in their recovery. I use this data and my analysis to refine the $\mathrm{CMOC}$ presented in Figure 9 which will represent how staff qualities worked at Woodlands.

\subsection{The Realities of Staff Qualities}

There was variability in service-users' experience of staff at Woodlands and there were challenges to therapeutic relationships being formed. When asked what they liked about being at Woodlands, some service-users identified the staff:

"the staff you know they will help you (.) they will go out of their way for you you know, and they are very respectful very nice (.) you treat them with the same attitude you get the same back" (Service-user Brian)

"they are very polite talking, they are very helpful when you are upset, they are more understanding" (Service-user Diana)

Both participants alluded to their experience of interactions with staff as positive and helpful. They both report that staff were respectful and understanding. These positive interactions suggest that these service-users experiences are in line with PAs aspirations of how staff would work at Woodlands. Brian suggested that staff would mirror how service-users treated them, suggesting reciprocity was a key part of the therapeutic relationships, which was a positive experience for him. However, this raises the question 
of how staff may respond to those who do not treat staff in a desired way. Whilst it is not explicitly clear who Diana is comparing the staff at Woodlands to, she suggests they are more understanding, particularly in times of distress.

Connie went further and identified that the acceptance she received from staff was important to her recovery:

"Service-user Connie: it wasn't until I came here that I started healing this place has healed me

Researcher: and what do you think about here has healed you then

Service-user Connie: just more so the the accepting people accepting me for who I am (.) people not telling me to be someone I'm not, it's not an us and them with the staff do you know what I mean like you get in some places (.) it's like they talk to you like they involve you in the conversation with them, and you feel accepted really (.) and that's important when you have a mental illness, to feel accepted that you are OK (.) that you are not a freak"

Connie positioned staff as different to how she has previously been treated and used examples of feeling the need to change, and there being a division between herself and staff, to represent this. Connie's account appears to align with PAs vision that staff have people skills and the ability to see the individuality within the service-users at Woodlands (see section 5.2).

Connie proposed that being at Woodlands, where the focus was not just on her mental illness, gave her hope:

"they look at all aspects of your life (.) I used to have a death wish you see, I used to be so scared about what lay ahead of me that I know I know I shouldn't be I felt so hopeless and I wanted to die really by drinking the red bull I tried to give myself a heart attack cause I am a vampire so it's too much protein for the heart so it destroyed my heart (.) but I made myself a new heart out of light cause I realise now life is worth living (.) so I guess you could say I am still mentally ill but I am not as depressed or hopeless as I used to be (.) so I guess I am in a better place" (Service-user Connie)

Connie's account appears to reflect her recovery journey from wanting to end her life and feeling scared about the future, to having hope and accepting her mental illness as part of her life. Connie referred to her heart being 'destroyed' and how she made a 'new heart out of light'; suggesting that the light Connie created took away the darkness and 
hopelessness in her life. The importance of hope has also been emphasised in service-user defined recovery (Deegan, 1988). Connie appeared tentative and unsure of where she was in her recovery, as she was still unwell, but no longer felt low or hopeless. This could suggest that Connie is still coming to terms with her new identity and outlook on life following her enlightenment.

Although the data referred to mainly positive encounters with staff, this was not reflected within all service-user experiences:

"they [staff] are always having a go at me for the way I talk to them and stuff like that, the way I talk is the way I talk I can't help that its part of my emotions I suffer with complex trauma (.) if you don't like the way I'm speaking to you or the way I'm approaching you you know you're paid to do a job (.) I said you're a nurse at the end of the day I'm a patient yeah, I'm living here yeah, basically the funders are paying your wages and they are expecting you to do your job and they're lacking a lot of capacity" (Service-user Simone)

Simone expressed that her actions were permissible since staff are paid to fulfil a role as a service provider. Her account could be read as a justification (Scott \& Lyman, 1968), in that she does not claim that the act was wrong, but instead attempts to explain why the act should be viewed as acceptable. Simone accounts for her actions towards staff as a consequential response to her trauma. Simone appears to orient towards funders to invoke a wider power and legitimise her account. Through foregrounding her trauma as an explanation, the account works to establish mitigation for her behaviour towards staff. Simone appears to orient towards a pattern of interaction that could be confrontational and instrumental. She suggests that as she was living at Woodlands staff should fulfil their role and do the job they are paid to do. This presents staff with a challenging scenario in which to build a therapeutic relationship to help with recovery, although a challenge that is not impossible. This suggests that for individuals like Simone, who may be more complex, or have experienced trauma, staff qualities alone may not be enough to develop a working relationship.

I have shown through my analysis of service-user accounts that their life experiences, and the complexity of their difficulties, can influence their relationship with staff. This 
highlights the challenges staff at Woodlands may face when attempting to build and maintain therapeutic relationships. This suggests that both the individual capabilities of the service-user and staff are crucial to relationship formation, and whilst desirable staff qualities may aid the formation in most cases, simply focusing on staff's qualities is inadequate. It is important to consider the capabilities and desires of service-users to positively interact with staff and form a relationship. I have used this data to revise the initial CMOC (see Figure 9) and have presented a new CMOC to account for this (see Figure 10). 


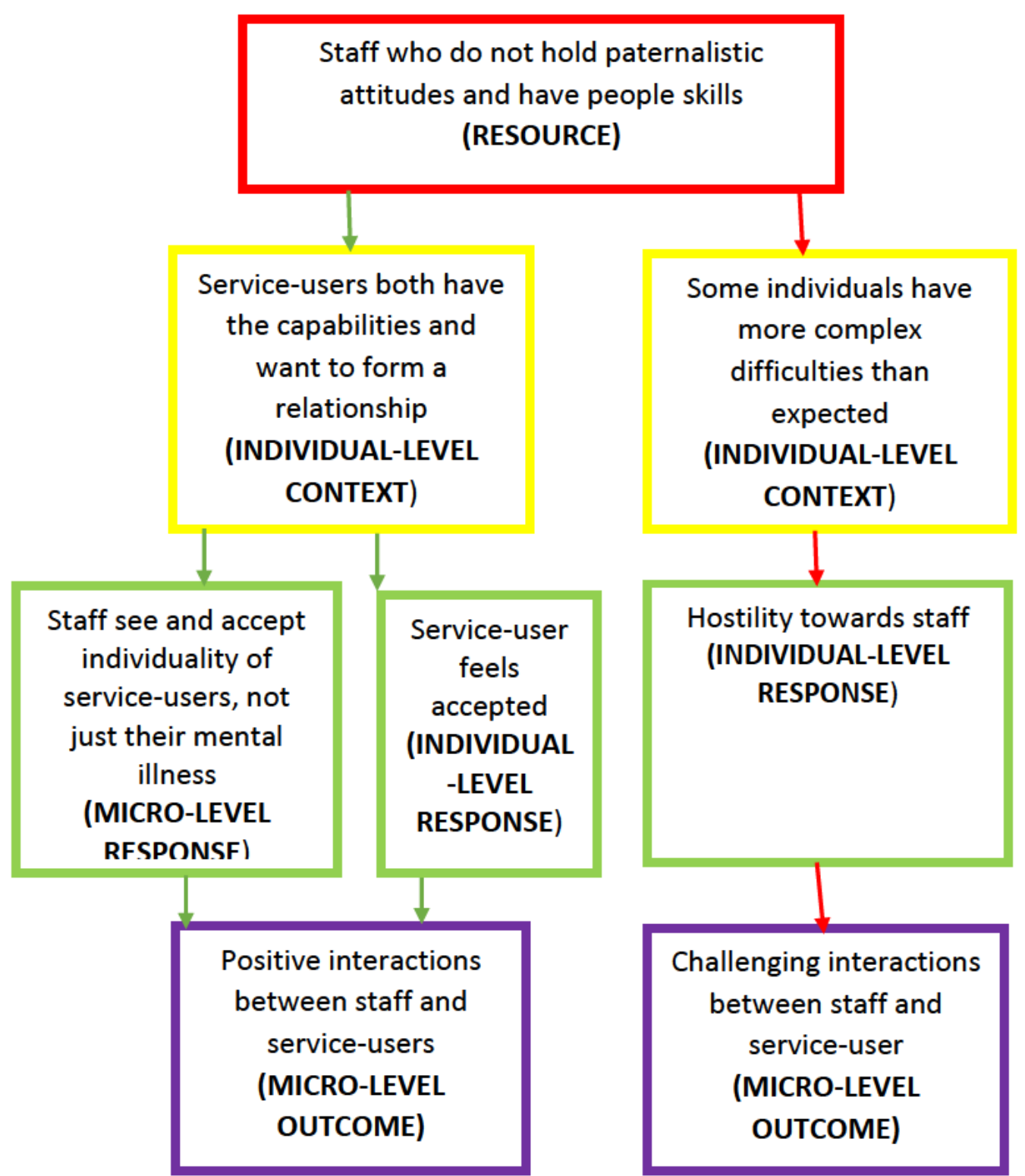

Figure 10 - Revised CMOC for Staff Qualities

Key:

CMOC - working as intended.

CMOC - not working as intended. 


\subsection{The Intended Staffing Levels}

Staffing levels were positioned as a unique selling point of Woodlands, but also as a means of providing enough therapeutic engagement. When asked about the development of Woodlands Joe identified the ratios of staff to service-users as part of the staffing model:

"looking at what is out there I think there is [service provider name] which are 16 beds which will have 1 registered nurse and staffing levels of perhaps 1 to 4 (.) if we were going to do that there was no point in us doing it (.) we were looking at the staff level of around 1 to 21 to 2 and a half so almost double that of comparator units" (PA Joe)

Joe refers to the higher staffing levels as signifying higher quality and positions this as necessary to deliver Woodlands' aims. Joe refers to other services which could suggest that the staffing levels were used to differentiate Woodlands from other providers. Later in the interview, Joe presented an alternative rationale for the staffing levels at Woodlands:

"so you know some people have ended up with behaviours, simply because there has been no other choice (.) you know in an institutional setting where I won't get to see anybody cause there's no nursing, there is 1 nurse in charge of 16, if the only chance I get to see the nurse is when I have a cut on my arm (.) so I cut myself, then I see the nurse"

(PA Joe)

Joe presents an extreme formulation to emphasise the benefit of designing Woodlands with higher staffing levels. He proposes that some behaviours may be a result of insufficient therapeutic engagement and uses this formulation to work as a direct contrast to what will be available at Woodlands. This suggests that there was an attempt by PAs in their design of the service to ensure service-users had enough therapeutic engagement available to them at Woodlands.

From my analysis of Joe's accounts, I have shown that the intended staffing level for Woodlands aimed to provide service-users with adequate therapeutic interactions with staff and to provide an alternative to what is offered by other providers. I note that this section was based upon one PAs account. Although Joe was the only PA who provided a detailed account of this component of the staffing model, it was still considered a key factor to the staffing model. I have used the findings of this section to inform the 
development of an initial $\mathrm{CMOC}$ of how the resource of staffing levels was intended to work at Woodlands (see Figure 11).

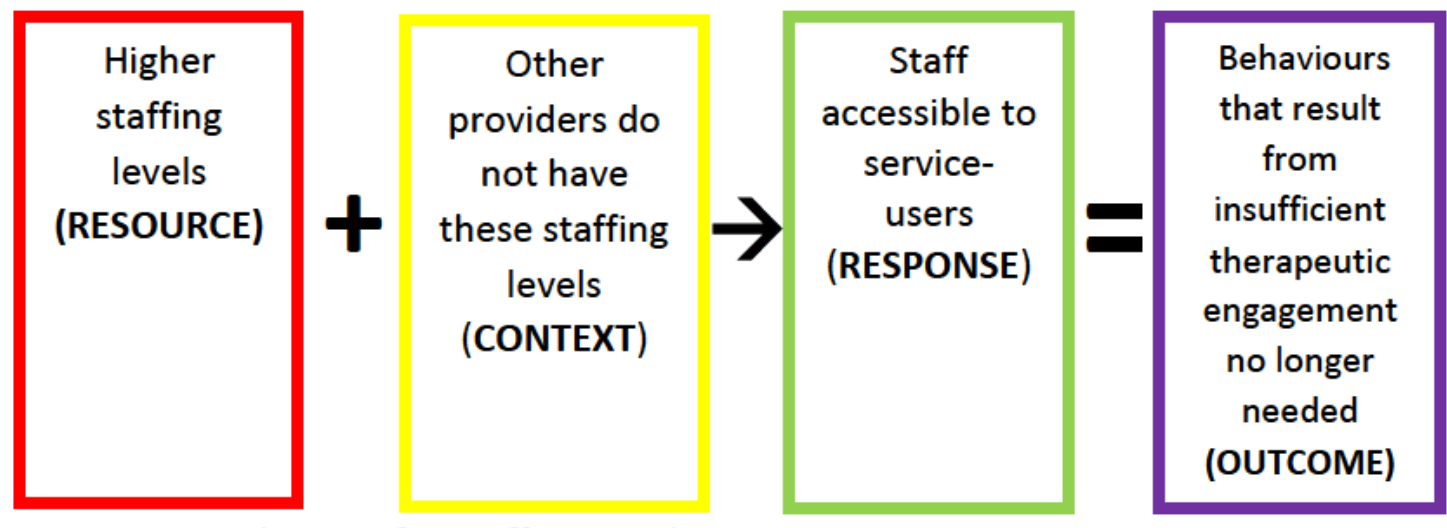

Figure 11 - Initial CMOC for Staffing Levels

\subsection{The Reality of the Staffing Levels}

The intended staff levels outlined by PAs in section 5.4 were influenced by the low occupancy, meaning there were unexpected outcomes:

"at the moment there is a community of staff but there is only three guests (.) I know that they know we are there to help them but I think the numbers don't really help I think it's a bit intimidating" (Staff Sophie)

Sophie refers to staff as ' $w e^{\prime}$ ' and service-users as 'they' which alludes to the dichotomisation of staff and service-users. The separation was further suggested by her reference to a 'community of staff'. This suggests that a process of coming together to take collective action had occurred without the inclusion of service-users, which deviates from the values of partnership working as described by PAs, (see section 5.2).

Ellen provided a description of her experience of the staffing levels at Woodlands:

"there was a load of staff just sitting round, and I just felt a little bit overwhelmed and I just kept thinking is there actually a guest or service user there (.) and they were just having a mothers meeting and I just felt a bit like well do you really have to be sitting in my living room having a mothers meeting when there is like 15 of you" (Service-user Ellen) 
Ellen referred to the space as her 'living room'; suggesting she may have viewed Woodlands as acting as her current home. However, it appears that this space was taken over by a large group of staff, which may have hindered Ellen's ability to use the space as she intended. Ellen suggests that staff were interacting amongst themselves, which could contribute to creating an us and them dichotomy between staff and service-users.

Ellen's account, however, contrasts with how Sophie described staff's behaviour in the context of higher staffing levels and low service-user occupancy:

"I think staff obviously erm feel at a loose end sometimes, particularly if you only have three guests and two of them may be on leave so there is one in the centre so you may have 4 members of staff and one guest you know (.) What do you do? There is only so much cooking and so much cleaning and paperwork that you can do" (Staff Sophie)

Sophie appeared uncertain of what staff were expected to do in response to the serviceuser numbers; which is unsurprising given the fact this was an unexpected difficulty, see Chapter 4. Sophie described that staff would complete daily living tasks, which was a deviation from the intended purpose of these tasks which were designed for service-users to complete (see section 6.4). This highlights a practical example of how the difficulties experienced at the exosystem, relating to individuals not being referred or accepted at Woodlands, influenced service delivery at the micro-level.

Sophie's account suggests that the tasks expected of staff were completed leaving them with nothing to do. Literature suggests that inpatient mental health staff often operate in busy, high-pressured environments, which restricts their availability and ability to meet individuals' needs (Muir-Cochrane et al., 2013). This highlights the unique position of staff at Woodlands who had time, which is often a limited resource in mental health service delivery (Coffey et al, 2019a). Despite being in a position where time was an available resource, staff did not appear to know how to use this, and perhaps oriented to taskbased working. This could be due to staff not wanting to inundate service-users, which could be staff responding to individuals reportingly feeling overwhelmed.

Mary suggested that the higher staffing levels had the potential for service-users to feel monitored: 
"I get it you don't want to be watched all the time (.) and I think when you are 4 staff and 4 guests you are watching everything all the time which isn't normal life either so the little things that maybe someone could get away with we see, so we react to it or have a conversation about it (.) whereas when there is 8 we are not going to witness every small little thing" (Staff Mary)

Excessive surveillance of service-users could be perceived as depriving individuals of privacy, intrusive and disempowering (Cox et al., 2010). Mary's account suggests that she was mindful about observation and recognised that it can be experienced as being watched by service-users. Mary suggests staff are likely to notice and respond to things, because the staffing levels afforded them the opportunity to do this. Her account therefore highlights a disadvantage in the day-to-day living of service-users when a service has been unable to match referral numbers with its planned staffing policy.

Although staffing levels were identified by PAs as key to the staffing model at Woodlands, staff and service-users identified other factors as more important to the therapeutic relationships. These factors related to the regularity of staff and the quality of these relationships.

Ellen proposed that the regularity of staff was important to feeling support was available:

"there is no regular staff on either like they have like one or two days in then a week off then like 3 or 4 days in then a week off, you don't see them for like 2 weeks (.) Did you just go to the Bahamas or something? I've got no one to talk to and your thoughts and feelings change every day and I I just like to project it out and be like rah rah rah and then its dealt with a problem shared is a problem halved" (Service-user Ellen)

Ellen makes the case that regularity and consistency may be absent due to the organisation of shifts at Woodlands. Her account suggests that there was too much variability in who was on shift, which prevented her from being able to access staff as a support mechanism. The absence of regular ward staff, or reliance upon temporary staff, has been identified as a source of upset for service-users elsewhere (Donald et al., 2015). This suggests that the issue of a regular staffing team may not be unique to Woodlands and may be more indicative of a wider system issue, which prevents services, like Woodlands, from operating with a consistent staffing team. 
It appears Ellen may not have built a trusting relationship with everyone on the staff team and may favour certain staff; so, when they are not around, she is concerned, or feels unable to confide in other staff. Her accounts highlight the challenges of forming relationships due to the inconsistency of staff but does show a potential opportunity for service-users, once they have built a close trusting relationship with staff, to be supported to generalise these relationship skills to others when needed.

When asked about the influence of staffing levels on individual's behaviour, Mary acknowledged that focusing upon the quantity of relationships available to service-users was insufficient:

"I think there are some [incidents] that are unavoidable (.) I think you could have 10 people on and you would still have some of the incidents that we have, erm, but then its hard to say (.) [service-user name] incident list is as long as my arm, but when she was in the acute ward it was treble, so is it just a change of environment? is it cause there is more staff around?... you are not going to get on with everybody, we hope that we forge good relationships but if you have 4 staff on and you don't actually have a good relationship with them its not going to make a difference" (Staff Mary)

She suggests that as a team they attempt to establish good relationships with serviceusers to promote therapeutic engagement. Mary was uncertain about why there had been a reduction in the number of incidents for this service-user, and presented possible contributors, which included staffing levels. However, Mary concluded that the quality of the relationship was more meaningful. Mary does not consider that the staffing levels may indirectly be important as service-users have more opportunities to establish a relationship with one staff member; and with more staff being there staff are less likely to be called away prematurely to attend to something else.

The resource of staffing levels was embedded into a context of low service-user occupancy. Due to this, staff had more time than anticipated, meaning they were left unsure of how to use this time, an "us and them" dynamic was created between staff and service-users, and staff deviated from the vision of Woodlands in promoting responsibility through daily living tasks (see section 6.4). I have used accounts from participants in which they claim that there have been several challenges and disadvantages of Woodlands being 
unable to marry its staffing model with its referral numbers. I have used my analysis of staff and service-user data to refine Figure 11 . The revised $\mathrm{CMOC}$ now includes the findings of how the resource of staffing levels was claimed to have worked at Woodlands (see Figure 12).

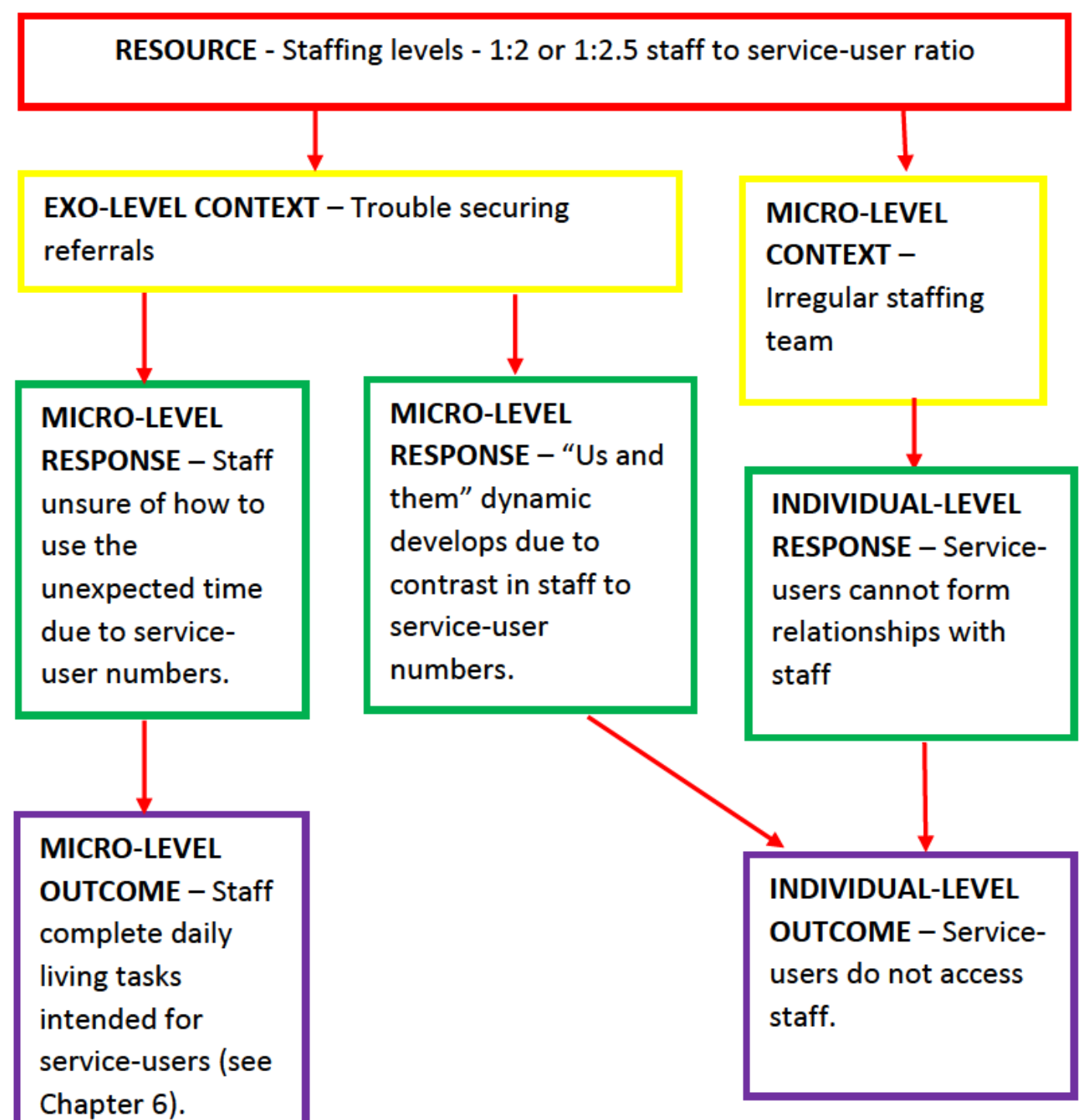

Figure 12 - Revised CMOC for Staffing Levels

Key:

CMOC - working as intended.

CMOC - not working as intended. 


\subsection{The Intention for Including Peer Mentors}

At Woodlands PMs were classified as individuals who have lived experience of a mental illness and had previous contact with services. PMs were identified as a key resource of the staffing group at the service.

I am aware that the term peer mentor may create a power imbalance between the PM and service-user - as mentoring is classified as a relationship where skills or knowledge are exchanged from someone with more experience to one with less experience (Dennison, 2010). I will, however, use this term as this is the term used at Woodlands. I will use the term professional staff to classify individuals who have been recruited based upon their objective, scientific knowledge and training, to distinguish staff from PMs.

When asked about PMs' role in service-user's recovery at Woodlands, Ethel suggested PMs would be a source of hope:

"I think it gives them hope (.) I think it gives them hope because obviously knowing that this person has had their own issues... has overcome their own problems to get to where they are today, it has to, it strikes a chord with people doesn't it?" (PA Ethel)

Ethel's statement aligns with research which has shown that meeting people who have found ways through challenges and difficulties relating to their mental health creates hope and belief of a better future (King \& Simmons, 2018). Ethel suggests that PMs will have 'overcome' their problems, which has been a criticised by Recovery in the Bin (2019) who suggest PMs 'are now expected to have acceptable recovery stories that entail gratuitous self-exploration, and versions of 'successful recovery' fulfilling expectations'. This suggests that there may be the potential for certain individuals, who are considered to have 'overcome their own problems', to be cherry picked as appropriate representatives of recovery.

PMs were positioned as individuals who would use their lived experience to support service-users:

"they [PMs] bring an insight of what it's like to receive services or care cause all of them have been engaged within secondary mental health services whether they have been an inpatient or received care, erm within the community (.) so they understand what it's like 
to be on the receiving end of a care and treatment plan (.) erm taking medication difficulties when you can't sleep or are struggling with your mental health, having good days and bad days so they understand that (.) whereas someone who hasn't been through that doesn't know what that feels like (.) so they can really empathise with someone but also demonstrate that there is hope after mental ill-health and also around the behaviours and taking responsibility for your mental health" (PA Elizabeth)

"where someone says to me you don't understand I may not (.) if someone says to a PM look you don't understand well it's like, well actually I do these are some of the coping things that I did" (PA Joe)

Elizabeth and Joe's accounts suggest that PMs are expected to use and share their lived experience. Through using their lived experience, it was assumed that PMs would be able to understand and relate to service-users differently compared to professional staff. Mol and Law (2004) suggest there are multiple ontologies of knowing about illness. Knowing about illness as an object, through objective, scientific knowing about the body from the outside, and as a subject, personally knowing about the illness from the inside. The latter encompasses the knowledge of PMs who have been socialised to the realities of living with a mental illness. Evans and Collins (2007) suggested that this form of expertise enables PMs to discuss and converse in a way that enables mutual understanding and commonality, which supports Elizabeth's and Joe's claims. It appears from their accounts that Elizabeth and Joe were mindful of the different types of knowledge about mental illness that can be included in the staffing model.

Elizabeth and Joe's accounts position PMs as being able to support service-users to take responsibility and learning to cope. This essentially reflects a medical model of health in that there is something wrong with you, and a PM can support you to be fixed. If PMs are working in a context which supports these values of recovery, then they may be functioning as paraprofessionals, which deviates from the true value of PMs (Mead et al., 2001).

Sandra goes further to suggest that PMs are uniquely positioned to understand knowledge from the perspective of both professionals and service-users:

"they [PMs] are the key link in the chain because while I admire our trained staff immensely in the job they do, the actual interface between the guests and our staff are 
those PMs cause they see both sides of the coin and they are hugely inspiration" (PA Sandra)

Sandra positions service-users and professional perspectives at opposite ends of the spectrum, and PMs will be operating within the liminal space between the two views. Her account suggests that PMs can create a bridge wherein the borders between 'our' (professional) and 'theirs' (service-users) can be crossed (Watkins \& Shulman, 2010, p. 171). Sandra appears to place the responsibility of improving this gap upon PMs, as opposed to understanding why this divergence between professionals and service-users was created in the first place. This raises the question of whether it is reasonable to expect PMs to take on the responsibility as agents of cultural change within mental health services (Gillard et al., 2015).

Her account suggests that professional staff may have limited face-to-face contact with service-users, which raises the question of what these interactions will be like and how regularly they will occur. This raises a further question of what professional staff will be doing whilst PMs are engaging with service-users and bridging the gap, and whether expecting PMs to be the 'actual interface' may blurred their role from PM to paraprofessional.

PA accounts show that PMs were intended to enable new forms of knowledge to be introduced at Woodlands, leading to hope and to bridge the gap between service-users and staff. It appears that PMs are encouraged to operate within a liminal space, where they are not a service-user, nor a staff member, which could place a huge burden upon PMs. I have used the findings in this section to inform the development of an initial CMOC of how they intended for the resource of PMs to work at Woodlands (see Figure 13). 


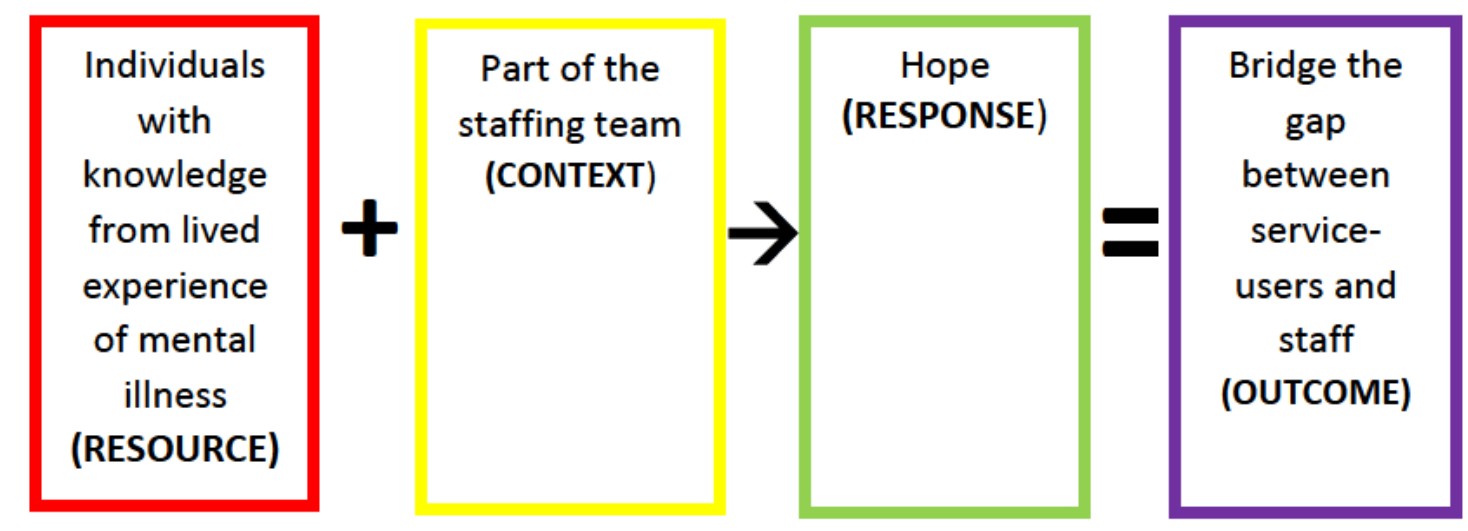

Figure 13 - Initial CMOC for PMs

\subsection{The Realities of the Role of Peer Mentors}

There were notable benefits and challenges of including PMs at Woodlands claimed within accounts by participants. There was also variability in how service-users responded to the inclusion of PMs as part of the staffing group at Woodlands.

\subsubsection{Being One of Them}

When asked about how PMs supported her recovery, Elsie identified that the similarities in their life experiences was beneficial:

"for someone to have gone through similar things that I have gone through, I mean I wouldn't wish it upon anyone but because they have been through it you can talk to them and they understand where you are coming from (.) erm, they can explain different techniques better cause they (.) from what they have learnt, so yeah invaluable" (Serviceuser Elsie)

Elsie's account suggests that she valued accessing the support of individuals who had knowledge of mental illness from their own experiences (Mol \& Law, 2004). Her account supports literature which suggests that this form of knowledge and expertise enables mutual understanding and commonality (Evans \& Collins, 2007). Elsie's description corroborates PA accounts that PMs could use their lived experience in their interactions with service-users (see section 5.6). Experiential knowledge of living with a mental illness has been noted as the psychosocial driver that underlines the success of peer support (Davidson et al., 2006), which aligns with Elsie's experience. 
Despite Elsie's positive experience of PMs, this was not the reality for all service-users. Simone did not see similarities between herself and the PMs:

"they [PMs] say we know what it's like (.) you don't know what it's like, you've got a job, you've got a house, you've got a job (.) you haven't been in hospital for the past few years (.) we've got fuck all, nowhere to go, so how can you say you're in the same position as us when you're fucking not so don't pull that one over us" (Service-user Simone)

Rather than focus on similarities, as anticipated by PAs (see section 5.6), Simone saw the differences between herself and PMs. The liminal identity of PMs may result in role conflict and ambiguity, meaning Simone only saw PMs as staff (Turner, 1967). Simpson et al (2018) also suggested that the liminal identity of PMs meant their different roles led to confusion about what their actual role was.

Self-categorisation theory (Turner et al., 1987) suggests people place themselves and others into social categories based on salient attributes. PAs assumed that because there would be individuals at Woodlands who identified as having lived experience (serviceusers and PMs) and several who do not (professional staff), the individuals with lived experience would identify with the social category of individuals with lived experience. However, other differences were identified as more salient to Simone, such as PMs having a 'job' and 'house' which meant PMs were not seen as like her. Later in the interview, however, Simone did acknowledge PM's mental illness and used this to emphasise her concerns about their inclusion:

"I find it [PMs] a risk factor to be honest, personally I'm not doing it to be discriminatory I just personally don't think people who have mental health problems should be working in the mental health sector themselves (.) I think people who have qualified as nurses they need to be physically and mentally trained they need to leave it to the professionals who have a clue cause they will end up having more relapses (.) cause there are some members of staff here who well, there is one member of staff who is not even on medication, I said hang on a minute is that a good idea cause he's working with vulnerable adults he shouldn't be working if he's not taking anything" (Service-user Simone)

Simone appears focused on role identity and wants individuals in concrete positions. This may suggest that PMs who operate in a liminal space and do not fit into a neat concrete 
identity may be challenging for Simone to accept. Her account suggests she values a more medicalised approach through her reference to 'medication' and wanting support from trained individuals. This suggests that Simone values knowing about illness as an object, which is achieved through scientific training, as opposed to knowing about the illness as a subject, which is achieved through lived experience (Mol \& Law, 2004). This could be due to the fact many services operate with a paternalistic narrative where professional staff's views are prioritised above others (Chen et al., 2013).

Simone appears to have internalised the stigmatising attitudes that she has encountered which she projects onto PMs, concluding that it is not safe for them to work at Woodlands. Farber (2006) suggests that if individuals disclose their mental illness, this may invalidate their perceived capacity to help and may cause service-users to worry about the individual relapsing. Simone's account highlights that how service-users perceive PMs influences the value and credit they give these individuals in terms of supporting their recovery.

\subsubsection{Working Differently to Professional Staff}

According to staff and service-user accounts PMs were able to provide something different to professional staff working at Woodlands. The category of PMs was used by Kuly to present a hierarchy amongst staff concerning their ability to interact with serviceusers:

"guests open up a lot more and feel a lot more safe and confident talking to you cause they know you've been through similar things" (Staff Kuly)

Kuly's account highlights a perceived difference between the communication abilities of PMs and professional staff. Kuly suggests that PMs had a different relationship with service-users due to their lived experience. It appears that the relationship between PMs and service-users seems to engender an opportunity that could have potential therapeutic value for both parties. This suggests that PMs have a unique opportunity to enter an interactional space with service-users which professional staff cannot do. 
Mary's account suggests that the experiential knowledge of PMs enabled a unique form of knowledge exchange, such as challenging service-users, which may not be achieved by professional staff who are working from their professionally acquired knowledge:

"we had a lady here who was peer support who built a really strong relationship cause she had the same diagnosis, and was on the same medication so they were able to discuss the pros and cons of it (.) but also she could challenge in a way that, well yeah I used to present like this and I got bored of myself, and I think oh wow that's a brave thing to say I could never say" (Staff Mary)

Her account highlights that tacit knowledge, which is derived from social means (Collins \& Evans, 2007), enables PMs to use an embodied understanding of illness based of their lived reality to interpret meaning and insight into the service-users current experience. Mary's account positions PMs experiential knowledge as distinctive from that of professional staff, which supports PAs vision of PMs bringing a different form of knowledge to Woodlands (see section 5.6).

Kuly presented a practice example to emphasise how she used her lived experience knowledge at Woodlands:

"somebody erm ligatured and erm, I found them and so I got them down on the floor and we were talking and then we were laughing (.) well when people came in and could see the person laughing they thought oh well it couldn't have been a serious attempt cause they were laughing and joking (.) but I understood it in a different way (.) when the emotions are so heightened it's such a serious moment sometimes the emotions can come out in a different way, so it might be crying or in this instance laughing it doesn't mean it wasn't serious at all, so I think it's more that knowledge we bring" (Staff Kuly)

Kuly is advancing her own expertise; she suggests she can see things that professional staff may otherwise miss and that she can handle these things in a different way that is potentially more sensitive to the service-user's experiences. Oborn (2019) also reported that PMs could understand and interpret the mental health needs of others in a way that differed from professional staff. Oborn et al (2019) attributed this difference to PMs being able contextualise the 'why' of behaviour based on their own experiences of mental illness, which aligns with Kuly's account. Kuly's example shows how PMs can work 
differently compared to professional staff and supports PAs vision for including them within the staffing model at Woodlands (see section 5.6).

Although, there were examples of PMs working different to professional staff, Walter suggested that professional staff's knowledge was prioritised:

"there has been a bit of a separation I think between the clinical staff and the more support staff (.) I think we are not always consulted on things" (Staff Walter)

Walter suggests that PMs were excluded from decision-making by the professional staff. Simpson et al (2018) reported that being ignored meant that PMs often felt like outsiders, rather than part of the team of people working together to support the service-user. It has been noted that whilst the inclusion of PMs is consistent with recovery values it does not by itself create the paradigm shift needed for ROC to replace a medical model (Farkas, 2007). If PMs are not involved in discussions or decisions then their inclusion may be tokenistic, as opposed to a genuine attempt to include service-user knowledge.

Walter also suggested there was a lack of awareness about the PM role:

"I feel as though one in particular is aware of my role and you know we can talk about things (.) the other 2 less so but I think they are less aware of the difference between my role and the recovery practitioners" (Staff Walter)

Walter felt he was able to provide something different to professional staff, however, had difficulties differentiating himself from them. Walter proposes that this was due to a lack of service-user awareness about the role. If PMs were unable to distinguish themselves from professional workers this could suggest that there was a blurring of roles, which could suggest PMs were functioning as paraprofessionals as opposed to as a PM (Mead et al., 2001). However, an alternative could be that regardless of their lived experience, service-users categorise PMs as staff, due to the significant differences between themselves and PMs.

PAs held the expectation that PMs would use their lived experience to support serviceusers, Kelly's account highlights that this expectation was not without challenge: 
"with any other job you work it's that sort of I can't really use my own personal experience or my own information but it took me a while to get used to it" (Staff Kelly)

Kelly appears to suggest that she needed time to transition into her new role as a PM where she was expected to share information about herself. Her account suggests that self-disclosure within an occupational context was a new experience for her. Selfdisclosure is defined as "the disclosure of inner feelings and experiences to another person" that "fosters liking, caring, and trust, thereby facilitating the deepening of close relationships" (Reis \& Shaver, 1988, p. 372). This highlights that the process of selfdisclosure is a dynamic interaction between two individuals; where one opts to selfdisclose, the other responds to this disclosure, and the first individual interprets their response (Reis \& Shaver, 1988). The willingness of individuals to self-disclose therefore rests upon their experience of this process, which highlights that self-disclosure is more complex than simply expecting someone to share their mental illness within the context of their occupation. Kelly's account highlights that there may be an extra burden placed upon PMs to use their own, potentially upsetting, biography. The expectation of selfdisclosure was not extended to professional staff, and in some circumstances, this may be actively discouraged in mental health settings; however, it is assumed that PMs will and should carry this burden. As there was an expectation for PMs to disclosure their personal recovery journeys, this may complicate their ability to provide mutual peer support as they are expected to offer some form of mentoring, or education in the position of a more knowledgeable helper; which deviates from peer support values (Mead et al., 2001).

Using my analysis of staff and service-user accounts I have refined the initial CMOC (see Figure 13), to capture how the resource of PMs was claimed to have worked at Woodlands (see Figure 14). 


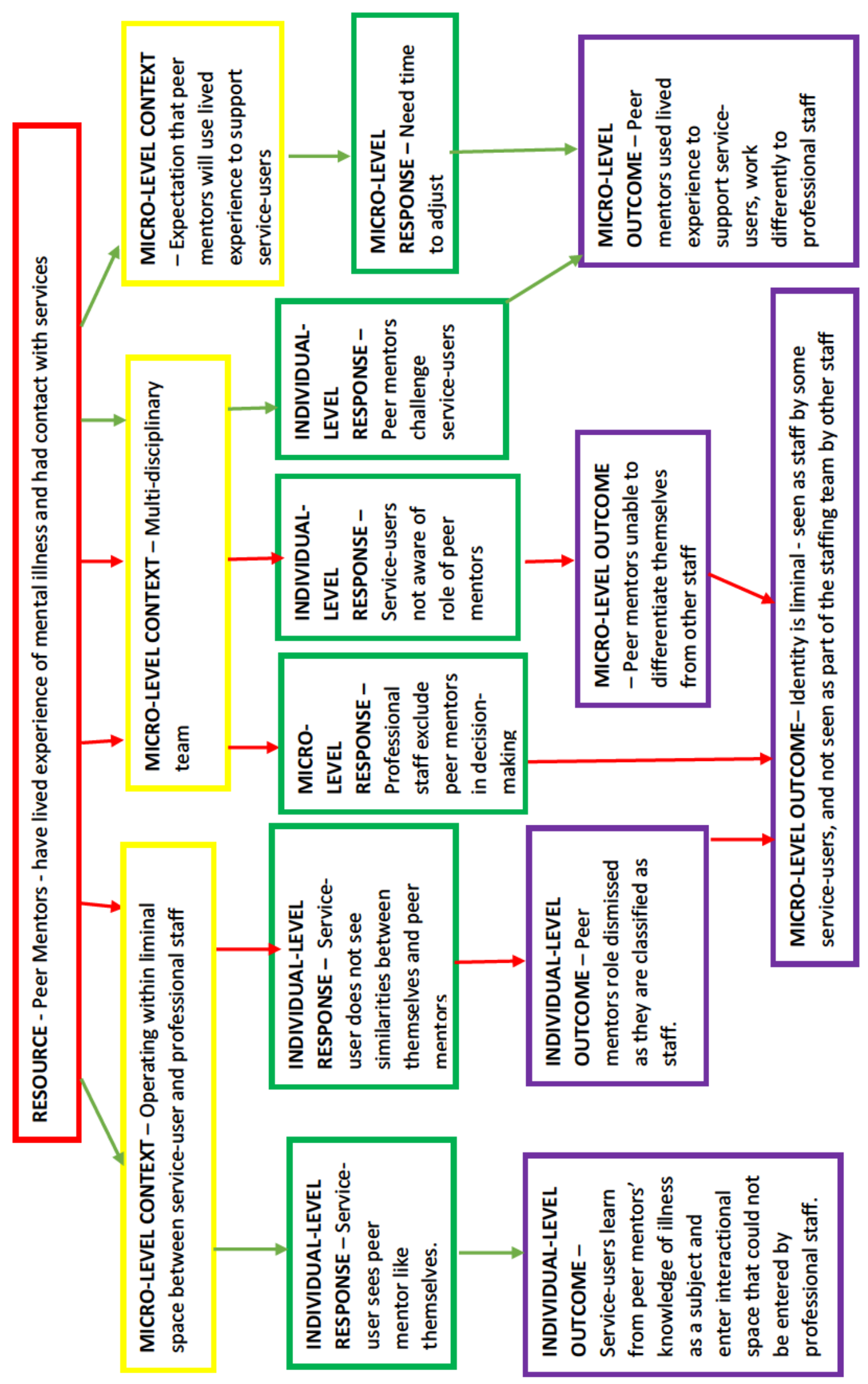

Figure 14 - Revised CMOC for PMs 
Key:

CMOC - working as intended.

CMOC - not working as intended.

\subsection{Summary}

This chapter highlights that PAs assumptions in relation to staffing characteristics, staffing levels and PMs were and were not operationalised as intended at Woodlands.

My analysis shows that service-users valued their interactions with staff, and these were generally positive, however staff did face challenges when establishing relationships with some individuals at Woodlands. These service-users did not necessarily fit the initial criterion proposed by PAs and therefore this could suggest that the qualities and skills of staff at Woodlands were not enough to support these individuals. This highlights the importance of staff being appropriately trained to work with all service-users accepted at Woodlands, as assuming their people skills would be enough was insufficient in practice. A substantial theory which supports these findings is the congruence model (Nadler \& Tushman, 1980) which suggests that organisations can only succeed when the tasks, the people, the organisational structure and the culture all 'fit' together - these findings show that there was not a good 'fit' between the staff working at Woodlands and their ability to complete their tasks of supporting these individuals in their recovery. It suggests that although Woodlands changed its strategy to accept individuals that would not have met the initial service-user criterion, it appears that the people component of the organisation did not have the appropriate skills to fulfil their task of working to support these individuals. This incongruence resulted in some service-users being unable to form therapeutic relationships with staff, staff feeling uncertain on how to support these individuals and therapeutic pessimism - outcomes that programme architects wanted to avoid with providing a culture of recovery where staff worked with the individual, not their diagnosis (e.g a personality disorder). 
As Woodlands was unable to match up their referral numbers with their intended staffing policy, this had negative implications such as service-users feeling intimidated, an us and them dynamic between staff and those accessing care, and staff deviating from other key visions of service delivery. This highlights the ripple effect of Woodlands not reaching its desired occupancy. These findings further emphasise the importance of there being congruence between the service and the wider system, which appears lacking at Woodlands. This provides explanatory power as to why the service had undesired and unintended outcomes and was unable to establish itself as a new provider.

PMs were able to use their liminal identity to communicate with service-users in an interactional space that professional staff could not access, this therapeutic engagement was highly valued by service-users. This highlights that PMs, if supported to fulfil their role whilst maintaining the values of peer support, can provide service-users with different knowledge and ways of working to support them in their recovery. My analysis shows that including PMs within the clinical team at Woodlands is more complex than PAs accounts considered. As PM's role was liminal, their identity was in limbo, meaning they were 'lacking a firm identity, [liminars] have shed their old identities and have not been given new ones, so they are neither one thing nor another" (Turner, 1967, p. 96). PM's liminal identity was shown to have both positive and negative responses at Woodlands, according to staff and service-user accounts. The positive aspect was that PMs were able to operate in interactional spaces that professional staff would be unable to enter. This meant that PMs could use their experiential knowledge to understand service-users' needs, engender openness and share a different form of knowledge and support. However, for serviceusers who valued concrete roles, the blurring of PMs' roles as both a service-user and staff created a sense of disdain. This suggests that the shared tacit knowledge of living with a mental illness was insufficient by itself in providing a source of connection for all serviceusers at Woodlands. 


\subsubsection{Explanatory Power}

In sum, the following partial, fallible theory suggests that:

- Where staff and service-users have the skills and desire to form a relationship, then the development of this bond can be aided if staff are accepting, kind and respectful towards service-users;

- Where services adapt their service-user criterion after hiring a specific staffing team, then staff may lack the skills, confidence and knowledge to support the new, unintended service-user group;

- Where services are unable to match up their staffing model with the number of referrals secured, then the presence of staff may feel overwhelming/intimidating, as opposed to accessible;

- Where service-users value smaller social settings, then challenges with referrals will be a positive experience, however if service-users value the social connection of other service-users then the limited numbers may be a lonely and isolating experience;

- When the organisational support is available to support peer mentors to distinguish themselves as a distinct role within the team, then peer mentors can work in ways which professional staff cannot through relying upon the mutuality of their experiences with service-users. If peer mentors are unable to distinguish themselves as different to the rest of the team, then they operate as paraprofessionals and cannot utilise their lived experience to support serviceusers in their recovery;

- When professional staff do not include peer mentors within decision-making at the service, then this prevents lived experience being embedded within the culture of the service, which is a key underpinning of the recovery narrative.

These findings emphasise the importance of identifying the right staffing group to support the delivery of the service, and to ensure that the appropriate structure and culture are in place to support these people to do their job. As the staffing group was developed to support individuals who were motivated and ready to work on their recovery, the 
workforce was not trained to support individuals with a personality disorder. This is interesting, as commissioners stated that this subset of service-users were the individuals likely to be referred to locked rehabilitation services at present. This suggests that Woodlands' strategy did not align with the inputs of who was being referred to such services to ensure that the staffing group meet these needs.

A key explanatory feature of the staffing model at Woodlands was the congruence between its work force (people) and the inputs of policy and commissioning relating to who is referred to a service like Woodlands. As stated previously, the congruence model states that when the work, the people who do the work, the organisational structure and the culture all ' $\mathrm{fit}^{\prime}$ ' together with the inputs, organisations are effective (Nadler \& Tushman, 1980), (see Figure 8). If they do not, as seen in Figure 10, Figure 12 and Figure 14 , such as peer mentors not being involved in decision-making and staff being pessimistic and uncertain about supporting individuals with a PD, then it makes it difficult for the ambitions and vision of Woodlands to be delivered as the workforce does not have the skillset to achieve it. The findings in this chapter suggest that the 'people' component of the model (see Figure 8) was not congruent with the skills needed to support the serviceusers accepted at Woodlands, meaning that some felt they were unable to fulfil they 'work' of supporting individuals in their recovery.

In Chapter 6, I present my analysis of another key principle of Woodlands' philosophy of care. This chapter will focus specifically upon choice and responsibility. 
6 FINDINGS - SERVICE-USER CHOICE AND RESPONSIBILITY 


\subsection{Introduction}

In this chapter, I present my analysis of research interviews with PAs, staff and serviceusers relating to choice and responsibility. I use two exemplars to represent the various practices that embodied and instantiated the service's values of choice and responsibility, and the claims of participants of how these were eventually enacted within practice. The two exemplars are choice as a learning opportunity and responsibility through completing daily living tasks (DLTs). The outcomes of interest were whether service-users made choices and whether service-users developed daily living skills for community living. This chapter relates to the interaction between the service-user and the microsystem. It considers how service-users were supported by those within the immediate environment to make choices and take responsibility, and how this related to their recovery.

A person's right to make choices without controlling influences has been widely advocated in mental health practice, as a key ethical principle (Lakeman, 2016). Choice has become a key feature within UK policy (DOH, 2003; 2007), with a focus upon serviceusers involvement in decisions about their care (Beitinger et al., 2014; Slade et al., 2017). Evidence from a systematic review indicated that control over aspects of one's life is important in recovery (Tew et al., 2012). However, it appears that collaborative involvement has been inconsistently implemented, with many service-users feeling uncertain of, or excluded from, decisions about their care (Metz et al., 2018). It has been argued that choice remains rhetoric rather than routine practice (Slade, 2017). Many service-users continue to report severely restricted personal agency and few genuine choices on how they live their lives (Milbourn et al., 2014). Taking responsibility has been considered a part of the recovery process (Anthony, 1993). This suggests that recovery entails service-users progressively assuming greater responsibility for their illness and life. There is a need for the locus of responsibility to shift towards the service-user, and away from staff who should inform, guide and do no harm (Lakeland, 2016).

I present my analysis of PA accounts first, as this is what was intended for Woodlands and will organise these findings into an initial CMOC. I then analyse staff and service-user accounts in which they claim how choice and responsibility were operationalised at 
Woodlands and will use these to refine the CMOC. This will allow for a transparent documentation of how the $\mathrm{CMOC}$ evolved through my analysis. 


\subsection{The Intention of Service-user Choice}

Choice was positioned as a key feature of service delivery and more specifically as a learning opportunity within PA accounts.

Elizabeth accounted for the importance of choice for service-users:

"the opportunity to have choice, people speak out, people will you know (.) guests will say well I don't really want to do that actually and they might not have had that opportunity before (.) or I would really like to do this (.) erm it's much more around empowerment and their self-determination and what they want for the future so being able to plan what they want (.) it's not actually being decided for them (.) they are actually deciding (.) this is where I'd actually like to go and its small steps" (PA Elizabeth)

Elizabeth presents two alternatives to choice, one where decisions are made by someone on service-user's behalf, and another where they make choices for themselves. Her account positions Woodlands as aligning with the latter, by providing service-users the space to voice their opinions and decide upon what they want to do in the present moment but also their future. Her account acknowledges that for some service-users this may be a new experience, but she does not explain how individuals will be supported to make decisions for themselves if they have not done this before.

Elizabeth also presented a detailed account of how choice could be a learning opportunity for service-users and staff:

"we've tried to look at that [the services response to service-user behaviours] in a way that is constructive rather than punitive, so it's not around punishment, it's around well what was behind that? What made you, what was your thinking at the time for that to occur and when you don't restrict then, that's quite a challenge for individuals cause that's the way they've been managed previously" (PA Elizabeth)

Elizabeth proposed that staff should not restrict or criticise service-user's choices and behaviours but should instead develop an understanding of its function. Elizabeth did not however present a detailed position of how this dialogue would benefit service-users. Elizabeth acknowledged that this approach may be challenging for service-users as it is likely to differ from the approach of other services. This suggests that PAs perceived Woodlands as providing something different from traditional care. 
Elizabeth's account is interesting as one commissioner wanted Woodlands to prevent service-users from being able to engage in potential unhelpful or harmful behaviours, such as substance use, to prevent an escalation in behaviour (see section 4.5.1.3). This suggests there may be a discrepancy between PAs intentions for managing the risks associated with choice, and how commissioners, who decide upon service-user placements, want services to respond to choice and risk. This may suggest that commissioners are perhaps wanting and purchasing more tried and tested services, and that the model of Woodlands regarding choice, challenges the idea of traditional services. Commissioners did not appear convinced by this alternative form of service delivery, and relied upon their experiences of what individuals need from services. This highlights the uphill battle that Woodlands encountered when trying to provide an innovative and alternative approach to service delivery as there is a strong normative counter pressure from commissioners to operate in a more traditional way.

In addition to service-users learning from their choices, Sandra proposed that they had to accept the associated responsibility that comes with choice, and make decisions that support recovery:

"you just have to trust people, and if they are unable to accept that level of independence and trust, then they will have to be discharged to a more secure setting... it all depends on, trust, partnership (.) we have to work in partnership with our guests but they have to work in partnership with us with the aim of recovery (.) recovery is not promoted for somebody with a serious mental illness by combining medication with drugs or drink" (PA Sandra)

Sandra's account appears to present recovery as a dichotomous outcome, which is at odds with the recovery literature. Recovery is not a linear process, and gains, losses, steps forward and backwards will occur (Deegan, 1988). The idea that someone may not be ready at a particular time, for any reason, is key. Whereas, Sandra presents an either-or scenario, where service-users either accept independence and trust, or they go to a more secure environment, which could be threatening to them.

Designing and structuring Woodlands in this way, which prioritises acceptance and readiness as a rigid marker of suitability, could work against the recovery process. Deegan (1988) suggested that individuals with a mental illness must be willing to try and fail and 
try again. Whereas Sandra's account suggests that if an individual is unable to accept or meet these expectations then this will result in them leaving Woodlands. Sandra's construction appears to position service-users as blameworthy when they are unable to meet these expectations.

Failure to meet this expectation can render individuals excluded and/or marginalised (Rose, 1996), which appears to be reflected in Sandra's account who suggested serviceusers could go to another setting. Sandra's reference to behaviours that may hinder recovery, such as substance use, could suggest that these behaviours are considered indicative of their readiness to accept responsibility for recovery. Her account omits the possibility that service-users may need to learn how to take responsibility and make choices that are supportive of their recovery, which may require the support of the staff.

Sandra briefly referenced a moral code for staff relating to their ability to trust serviceusers (Foucault, 1985). However, she did not mention any potential outcomes or consequences if staff were unable to fulfil this expectation. Instead, the default position related to relocating any service-users who do not behave in a way that aligns with Woodlands' expectations. The problem here is this may inadvertently signal to serviceusers that the power lies with staff, which could undermine the partnership working Sandra referenced.

Sandra's account could be considered as rhetoric, rather than the detailed position of how choice and responsibility would be achieved at Woodlands. This was reflective of most PAs accounts which suggests they may have seen their role as laying out the broad philosophy of Woodlands and that they expected practitioners to work out the means of achieving it. Whilst this is not unreasonable in a research interview when called to account for decisions previously made, it does raise the question of how these ideas were conveyed to staff and what support was enabled to operationalise these visions.

Joe and Elizabeth both recognised that choices can lead to mistakes, but wanted to leave space for service-users to make bad choices as this would provide an opportunity for learning and growth: 
"but actually somebody's wrong choice might be exactly what they need because, they may need to have a wrong choice, something go wrong and they learn from that, develop" (PA Joe)

"to allow that person to make mistakes and I think that's the difference (.) we all make mistakes and someone with a mental illness will make mistakes and they need to be able to do that and learn from that" (PA Elizabeth)

Both participants alluded to the need for therapeutic risk-taking, considered as the opportunity to take a course of action that may have an adverse outcome (Stickley \& Felton, 2006). Elizabeth attempted to reinforce the importance of risk-taking by normalising that everyone makes mistakes and that individuals with a mental illness should not be deprived of this opportunity.

Joe and Elizabeth omitted to present a detailed position of how staff and service-users will be supported in therapeutic risk-taking. Joe and Elizabeth did not populate the categories of good or bad choices; however, it would be expected that both held preconceived ideas about what constitutes a good or bad decision, which may inform how they would expect staff to respond to service-user's choices. Neither referred to there being a limit upon the leeway afforded to choices or therapeutic risk-taking. This shows that the operationalisation of choice as a learning opportunity was left open to staff interpretation as choices will depend upon a range of information that can only be sourced within practice.

My analysis of PAs accounts relating to choice forming part of Woodlands' values shows that there were inconsistencies within PAs accounts as to whether choice would be positioned as a learning opportunity, or if certain choices such as taking substances, should have consequences, such as losing their place at Woodlands. The discrepancy between PA accounts of how choice should be positioned at Woodlands shows that there was not a consensus for operationalising choice. This has the potential for staff to be provided with mixed messages of how choice should be promoted and encouraged at Woodlands. I have used the findings from this section to inform the development of an initial CMOC of how PAs assumed choice would work as a learning opportunity at Woodlands (see Figure 15). 


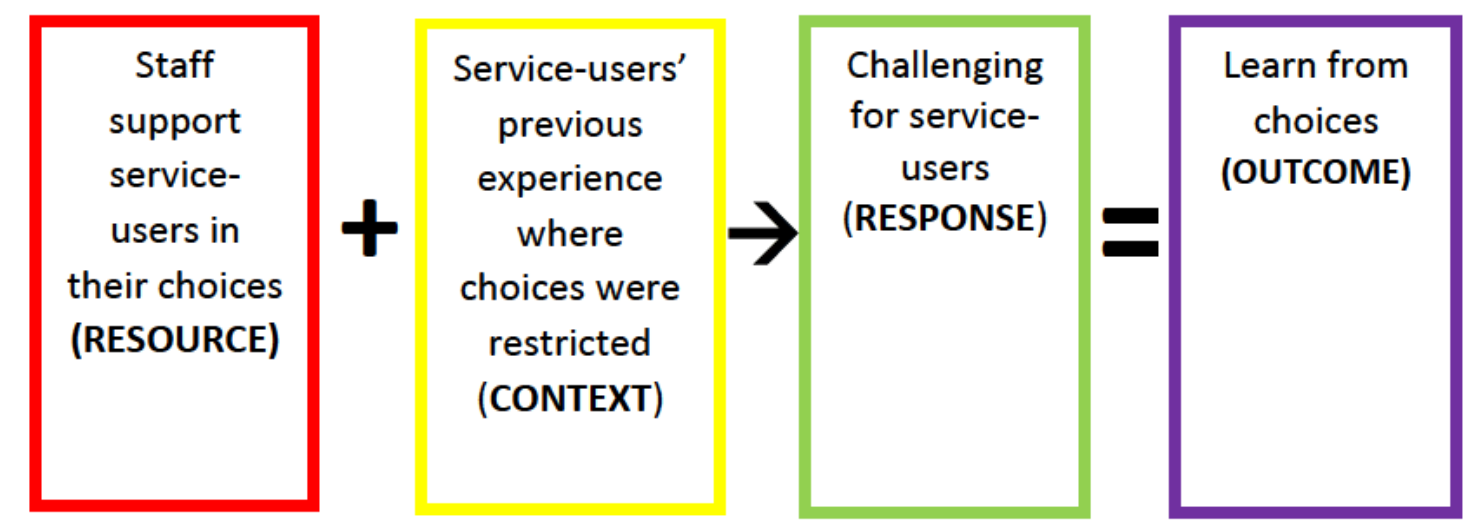

Figure 15 - Initial CMOC for Service-user Choice

\subsection{The Realities of Service-user Choice}

Staff and service-users accounted for how choice was positioned as an opportunity for learning and growth.

Kuly suggested there were restrictions which prevented staff from being able to support service-users in all their choices:

"they can get frustrated because if they want to do something that we can't encourage (.) like the pub (.) they are allowed to but we can't encourage that" (Staff Kuly)

Kuly's account suggests that the support of choice was influenced by whether staff determined the choice appropriate. Kuly identified the 'pub' as an example of a choice that could not be encouraged. Kuly's choice of going to the 'pub' is interesting as this suggests that what is considered normative at Woodlands, may be at odds with what is considered normative within society. Accessing the pub could help service-users to learn how to go to the pub without excess and develop social relations that extend beyond inpatient care. However, rather than viewing the choice of going to the pub as a learning opportunity, as intended by the PAs, Kuly felt unable to encourage this in her role. This could be due to the professional responsibility of staff to ensure service-users, staff and the public are not at risk of any harm, and therefore staff are risk-adverse to potential harmful situations (Chen et al., 2013). 
Her account highlights that staff who may be reticent about encouraging certain choices may be unwittingly restricting the facilitation and support of service-user choice. Kuly's account suggests there is a potential for a struggle between service-users who wish to exercise control over their life, and staff feeling able to encourage their decisions. PAs left the operationalisation of choice open to those delivering the service, and it appears that a blanket rule has been applied by some staff. Kuly's account fails to think about the individual service-user or weigh up the benefits and costs of going or not going to the pub, and to devise a plan so they are supported to do this safely. This application of choice limits the opportunity for service-users to learn from their choices.

Kuly's account suggested that the 'pub' could not be encouraged, however, another staff member suggested that the pub was being used as a form of leverage to demand serviceuser compliance "it [the pub] is dangled in front of people as a reward which I think is horrific personally you know (.) do this and then you can go to the pub for a pint (.) I think that's awful" (Staff Thomas). This highlights that staff may have different views of the degree of risk posed, when to intervene, and under what circumstances. As discussed in section 6.2, PAs left room for staff interpretation concerning how to operationalise choice as a learning opportunity at Woodlands. Whilst this is arguably necessary so staff can flexibly adapt these principles to the context and individual service-user, it appears that it was left to individual interpretation and application, as opposed to a staff consensus. It appears there was no agreement amongst frontline staff on what decisions were, or were not acceptable, which resulted in an inconsistent application of the principle of choice at Woodlands.

Thomas presented a hypothetical example of how service-user choices may initially be beneficial; however, the long-term benefit of repeating certain activities on a regular basis was questioned:

"Costa, I mean I think it's fine and it can be very helpful for, so to give an example (.) if someone is suffering from extreme anxiety and they don't want to leave the house... the first day they step foot out their house that's a really big jump, that's a big step (.) after they've been doing that for a month that's not so much of a big step, you know the goalposts need to be moved and people have to progress so I think if someone is going to Costa every day maybe initially it's really good for them (.) it's really helpful erm, it gives them something to do, have a chat, people watching, I think there are loads of good aspects to going for a coffee (.) erm (.) I think every day is a bit ridiculous" (Staff Thomas) 
Thomas' account suggests that going for a coffee is a step, but if there are no more steps to follow this then little is achieved. Thomas appears to see limited value in participating in certain activities every day and highlights the need for variability for service-users to continue to benefit and learn from their choices. Thomas recognises that it is unusual for people in communities to frequent the same places every day and not see themselves as stuck but engaged in that community. He implies that further progress needs to be planned so that people, including staff, do not get stuck in thinking they have achieved all they need to. Thomas' view on what constitutes a meaningful activity does not appear to be shared by all other staff as going for coffee was regularly facilitated.

Meaningful activities within inpatient services have been identified as a quality standard in NICE guidelines (NICE, 2012). Although meaningful activities have been discussed in literature and embedded within policy, what constitutes and defines a meaningful activity is not clear (Hendryx et al., 2009), which could go some way to explaining the variability seen within staff's understanding.

Patricia claimed service-users had difficulties making choices at Woodlands:

"we had a very very interesting erm issue with that in the beginning, cause we were like right we are going to let guests make the choice (.) but they had come from somewhere where they had very little to no choice, to too much choice, and they were so shellshocked by it they didn't know what to do" (Staff Patricia)

Patricia attributed the difficulties service-users faced when making choices to their previous experiences of mental health services. Service-users may have had limited opportunity to make choices, and her account recognises that they may not have the skill or experience to make choices. This highlights that PAs idealised vision, when met with the reality of delivering services to individuals with complex needs, required reworking and reshaping by staff on the ground. It appears that PAs optimism may have overestimated service-users ability and readiness to making choices independently. Patricia's account does, however, communicate that the decisional power rested with staff, as service-users were being allowed the opportunity to make choices; suggesting this chance could be revoked by staff. 
Rather than adopting an agreed team approach on how to promote choice, it relied upon individual staff interpretations. It appears that there was no agreement between staff on what constituted acceptable or unacceptable decisions, which resulted in an inconsistent application of choice at Woodlands. Regardless of this inconsistency, staff accounts suggested that although choice may have formed part of service delivery, staff were able to revoke this, and the decision as to whether choices were facilitated ultimately rested with staff.

\subsubsection{Tension Between Promoting Choices and Service-users Needing Support}

Service-users expressed that they wanted and needed staff support to learn how to make choices. Ellen, a service-user, suggested that she was not prepared or able to make decisions by herself:

"when I first came here it would be like well one guest goes and does that thing and what would you like to do? It's not like you can join in with theirs cause it's their idea do you get what I mean, and I just thought you know, share the ideas out (.) sharing is caring so yeah it was a bit overwhelming then cause I had to think of something to do (.) surely you are the ones getting paid to er to be here to help with the recovery so why aren't you chucking out some ideas out, so that did pee me off a little bit that did"

(Service-user Ellen)

Ellen indicated that she wanted support from staff, or even other service-users, to make choices, yet this did not appear forthcoming in practice. It appears that staff may expect too much of service-users when it comes to making choices. Service-users can, however, be helped to build these skills again, but leaving individuals to make their own choices without support may mean they are unable to make use of this opportunity. This account represents an example of when the balancing act rested too much upon service-users being self-reliant, as opposed to staff supporting them to become independent.

This account highlights that PAs and staff may have overestimated Ellen's ability and skillset to make choices; meaning Woodlands' approach to choice did not meet Ellen's needs. It could be argued that if Woodlands is aiming to provide ROC, service-user's ability to make choices would need to be assessed to ensure each individual was supported to 
make choices at their own pace. However, Ellen's account suggests there was a one-sizefits-all approach to choice, and if they did not fit with this approach then there was no alternative. This may suggest that the service's philosophy towards choice was rigid and non-negotiable, limiting service-users' opportunities to learn how to make choices and engage with this opportunity.

Ellen provided her account of what happened when she was unable to make choices and staff did not support her:

"if you don't come up with the suggestions then nothing gets done and that is the frustrating point and like, er, a couple of, a guest who has been here for quite a while she goes off and does stuff (.) and like even she has been saying oh I'm a bit bored now, what's there to do? And I'm like, sometimes I'm like how have you been here for a year and a half? Do you know what I mean... how have you not lost your marbles? I've been here for 3 months and sometimes feel myself not getting better, and just getting frustrated cause I am hanging out just not doing anything" (Service-user Ellen)

Ellen suggested that staff left service-users to make choices about how they wanted to spend their time. If service-users were unable to make choices Ellen suggested nothing was facilitated. Boredom and having little to do have been identified in several studies (Chen et al., 2013), with inactivity being associated with reduced self-efficacy and exacerbated symptoms (Muir-Cochrane et al., 2013), which Ellen alluded to. Her account suggests that service-users need an objective beyond simply occupying themselves, or else they were working towards something with no specific outcome.

Diana proposed two factors for not wanting to make decisions for herself:

"in my last unit we never had the choice of what to do (.) so here, I am still trying to get over it, it's quite hard to accept (.) I don't really like choosing what to do, cause I have never had to choose what to do, so I would rather people chose for me (.) I know that's the wrong choice but at the minute, until I am ready" (Service-user Diana)

The first factor related to her limited experience of choice in previous placements and the second was the discomfort of accepting the responsibility that comes with choice. Some literature suggests that although choice has become a critical sign that we have freedom and autonomy, choice can become excessive, leading to paralysis and avoiding decisions entirely (Schwartz \& Ward, 2004). It has been suggested that individuals who have been 
in long-term restricted environments become dependent upon services, lose confidence to make decisions and lose a sense of being an independent agentic individual (Goffman, 1961; Chow \& Priebe, 2013). This is arguably seen in Diana's account as she does not appear to like, or feel comfortable, with the possibility of making choices and instead opts to leave this to someone else. This has been seen elsewhere when delivering ROC within inpatient contexts (Waldemar et al., 2018a; 2018b). Although Diana preferred to rely upon staff to make decisions for her, she did introduce that she was open to the idea of making choices, but at this point in time she felt unprepared. This highlights that Diana needs staff support to feel comfortable to start making choices for herself.

Staff also suggested that service-users with limited experience of making choices were ambivalent towards choice and would deflect responsibility back onto staff:

"for people who have not had a choice, you get a lot of I don't know, I don't mind, it's up to you, and they try and push it back onto you and they find it very difficult to make a decision cause they don't know if it is going to be right or wrong" (Staff Mary)

Mary's account implies that service-users choice can be right, or wrong, which suggests staff are judging their decisions. If service-users are aware of these judgements, they may seek to avoid making choices where possible, preventing them from learning and being independence.

Another example showed that some staff did not know how to support service-users who made certain decisions, such as not engaging:

"this is the thing that I sort of wrestle with myself in terms of how much sort of (.) how much persuasion we should be using (.) cause at the moment if someone doesn't feel like doing anything, or a particular thing, then we just go oh ok then fine, and then it ends there" (Staff Walter)

Walter's account alludes to service-users' needs for support being balanced against the structure of a service that promotes the 'responsibilisation' of service-users (Cradock, 2007). His account suggests staff opted to back away in response to service-users who chose to not engage. This suggests that service-users were left with the responsibility for motivating themselves to engage in their recovery. Walter was not sure of his role in 
supporting service-users who chose to not engage, although he had an awareness that doing nothing was perhaps not the most appropriate response to supporting these individuals.

Hungerford and Fox (2014) reported that staff adopt a hands-off approach as they interpret the recovery model as service-users doing it all for themselves. This raises the question of whether neoliberal ideals of self-management and self-reliance are prioritised over the needs of service-users and ensuring the appropriate support is available. Walter's uncertainty of how to support service-users who specifically lacked motivation or engagement could be because Woodlands was intended to support individuals who were motivated and ambitious (see section 4.4). This suggests that the resources and skillset to support service-users who chose to not engage may not be present at Woodlands.

From my analysis of participants accounts I have shown that some service-users were not able to make choices for themselves for several reasons and they wanted and needed the support of staff to develop the skills to make choices. This highlights that staff had to negotiate how and when to support service-users to develop the appropriate skills to make choices, as well as giving them autonomy.

\subsubsection{The Role of Social Processes in Choice}

Social problems and processes directly impacted service-users ability to engage and act upon their choices, which has been reported in other studies that found that social issues can influence psychosocial outcomes and recovery (Ungar, 2013). Mary referred to the negative experience of one service-user when attempting to access an educational course of their choosing:

"with the college course that was a difficult one really cause that's when you realise there still is stigma (.) erm, we had er, we had a guy who did a college course in [location name] didn't we and erm they noticed that he had a diagnosis of schizophrenia so he had to have an automatic risk assessment done discussing everything and I just thought this wouldn't happen for everybody (.) whereas his mental illness had nothing to do with what he achieved at college and they completed a risk assessment with him and they never got back to us they never returned any phone calls nothing" (Staff Mary) 
Her account suggests that she did not acknowledge the potential influence of macro-level processes. This is interesting as there is a robust body of evidence that demonstrates that discrimination and stigma influences nearly every domain of an individual's life (Thornicroft et al., 2007; Sharac et al., 2010) and can restrict individuals in their recovery and treatment seeking (Corrigan, 2004). However, staff appear to have suspended this knowledge and appeared surprised when they encountered such attitudes.

Mary assumed that the college's response was due to the service-user's mental illness. This highlights that although Woodlands may encourage choice, service-users ability to act upon these choices were restricted by wider societal barriers. This highlights the interdependencies of social systems operating at different levels, suggesting that the operationalising of Woodlands philosophy towards choice was influenced by wider structural processes. Mary's account highlights a pattern of disadvantage that occurs for people with a mental illness, which is referred to as structural violence. Structural violence refers to inequities and injustices embedded in social and institutional structures within societies (Farmer, 2004) and which have been shown to differentially apply to people with enduring mental ill-health (Kelly, 2005).

Mary's account highlights the need for a focus upon broader, societal change to support individuals in their recovery (Recovery in the Bin, 2019); and to address the structural and attitudinal barriers to social inclusion and participation (Baumgartner \& Burns, 2014). Mary's account shows that service-users experienced discrimination and stigma therefore providing services that focus solely on individual change fail to address the substantial social disadvantages and inequalities that people with mental health conditions experience day-to-day. Whilst societal change is not within the capabilities, or remit of Woodlands, it does highlight a challenge the service faces when attempting to promote and support choice and to help them establish their role within the wider community.

This negative experience changed staff's behaviour when identifying and supporting service-users in making choices and accessing community-based opportunities. Staffs' response was to encourage opportunities that were more inclusive and aware of mental illness: 
"we [the staff at the service] try our hardest to identify placements that understand mental health because there is the issue of rejection and we have had that unfortunately... if we go down [organisations name] they know they are safe... its run by peer mentors who understand it there is less chance of rejection, and I think that's the safest way to get back into the scary groups... I know I am more cautious about it" (Staff Mary)

Mary's account suggests that she was mindful of the need to weigh up the risks of rejection versus the possible benefits of wider engagement beyond the usual groups with mental health awareness. Her account suggests that as a result of negative experiences service-user opportunities were restricted to those within the mental health sphere, which has been seen elsewhere within literature (De Ruysscher et al., 2020). This prevented service-users from having contact with non-mental health agencies, which could be valuable form of support for them. Whilst Mary justified this decision as a means of protecting service-users from future rejection, avoiding mainstream services could inadvertently penalise and restrict service-users access to a diverse range of opportunities.

Referring service-users exclusively to resources intended for individuals with a mental illness could be considered as ghettoisation (Durocher et al., 2014; Stewart, 2019). Ghettoisation is defined as 'to put in or restrict to an isolated or segregated place, group, or situation' (English Oxford Living Dictionaries, 2020). Although restricting service-users to mental health-specific opportunities was not a deliberate attempt to ghettoise or marginalise individuals, it may foster this mentality. Due to this, service-users may feel they are not capable of operating or belonging to mainstream society, as their social ties are restricted to those within the mental health sphere (Stewart, 2019).

Her account suggests that staff adjusted their expectations for individuals based upon the feedback they get from their encounters with other organisations, and as a result of this adaption to what staff think is possible, service-users are marginalised from participating in community life. Changing Woodlands' approach to choice may contribute to structural violence, as discriminatory practices are inadvertently legitimised and reinforced. This change to the service's practice of supporting individuals to act upon their choices could 
serve as a barrier to the sought-after goal of service-users' integration into the community.

Elsie, a service-user, felt the need to justify why individuals needed access to the community:

"at the end of the day if we are going to be moving back to the community we need to learn how to live in the community so we need that access at the end of the day (.) we are people we are not dangerous animals that can't be let out (.) we need to be let out, we need to do things or else we will just get institutionalised" (Service-user Elsie)

Elsie's account works to humanise her situation and that of other service-users to defend their need for community access. Her account invokes a common trope in the form of an image of wildness, uncontrolled and untamed animals versus people, recognising that public fear may be part of the issue of acting upon her choices. Elsie seems aware of stigmatising beliefs about individuals with a mental illness being dangerousness (Corrigan \& Rao, 2012) and that these beliefs about mental illness contribute to institutionalisation. Her account highlights an awareness that although staff may view service-users as individuals (see section 5.2 and 5.3), this perspective may not extend to the wider community.

Choice needs to be coupled with an awareness of the social processes that have been identified from my analysis as being influential in the plotting of recovery journeys. This highlighted that choice is only one part of the recovery journey, and this component is not enough on its own. In addition to awareness of social processes, service-users require support to develop the confidence and skills needed to be able to make choices for themselves. I have used these findings to refine the initial $\mathrm{CMOC}$ (see Figure 15), to represent how choice was operationalised and working at Woodlands (see Figure 16). 


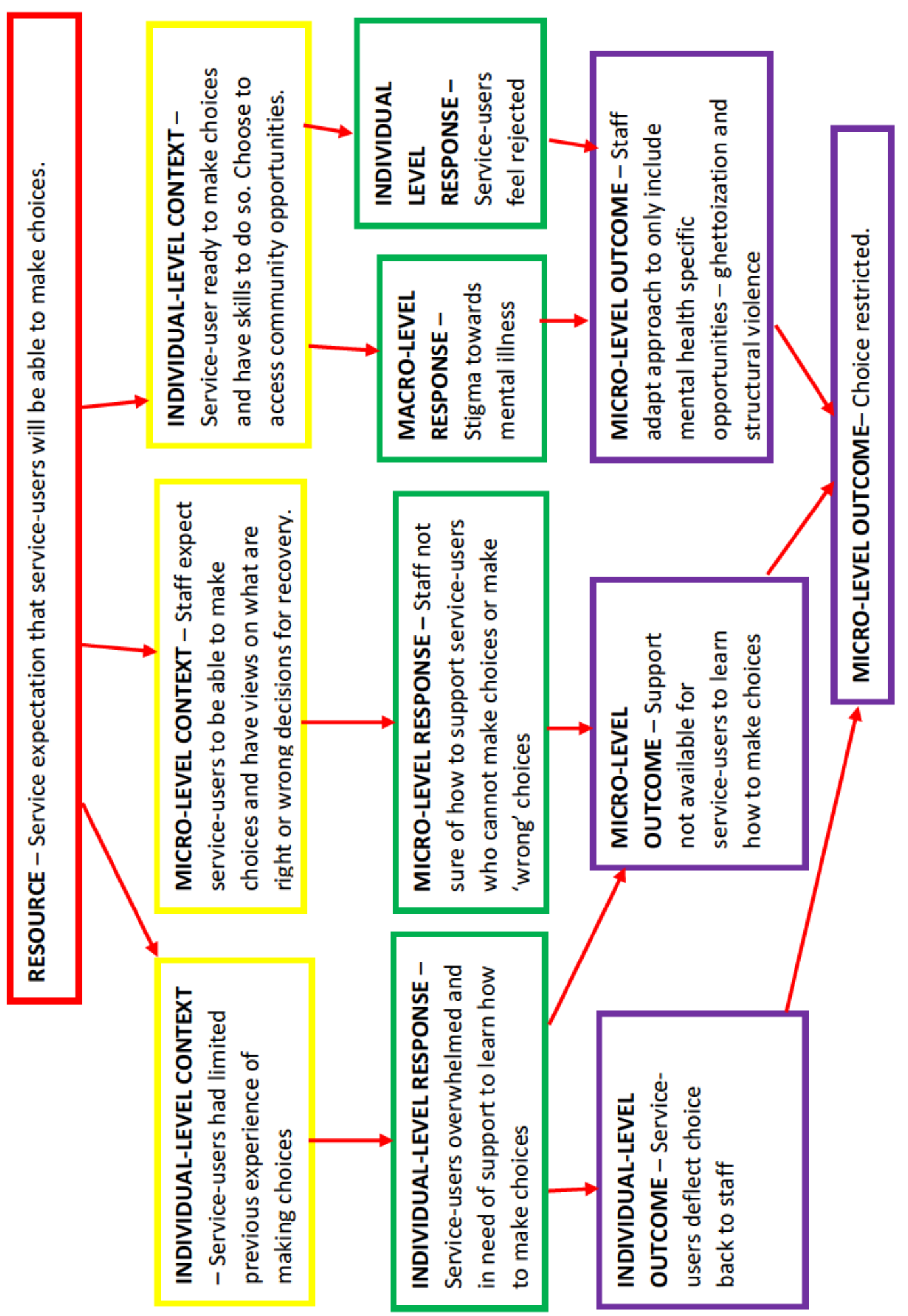

Figure 16 - Revised CMOC for Service-user Choice

Key:

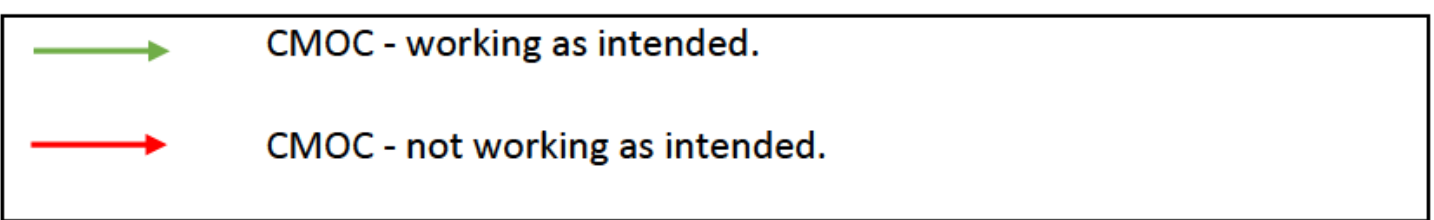


In the next section I shift my attention to focus upon the second part of this chapter which relates to service-users taking responsibility for the completion of daily living tasks.

\subsection{The Intention for Service-users to Take Responsibility for Daily Living Tasks}

Daily living tasks (DLT) were an important mechanism for supporting service-user responsibility at Woodlands by PAs. The predefined DLT were associated with the skills needed for independent living:

"well it [service-users taking responsibility for cooking and cleaning] covers an awful lot really, cause it's about what we've got in the cupboards, what might we need to buy, have we got enough money to buy it, it's all the budgeting (.) it's all the things you would have to do if you were living on your own (.) it's what you're going to eat today cause I haven't got much money so how am I going to make something out of nothing erm, and er, so it's all of those daily living skills that develop but those are part of the everyday"

(PA Elizabeth)

Elizabeth framed the development of these skills as beneficial for community living. The philosophy of independent living was based on the social model of disability and the idea that social structures disable people from full and meaningful participation in communities and activities of their choosing (Charlton, 1998). The notion of selfdetermination underpinning the independent living philosophy does not assume that individuals can necessarily achieve this alone, but with assistance if needed (Morris, 1997). Therefore, it is not necessarily about service-users doing things for themselves, but in having control over their day-to-day life. For many, having support is an essential precondition for overcoming social barriers and realising their right to live independently and be included within the community.

Literature has suggested that service-users have difficulties in looking after themselves upon discharge, which supports Elizabeth's account of the need and importance of supporting individuals in developing these skills (Owen-Smith et al., 2014). This suggests that although there is a need to assist and support service-users in developing skills; 
independence should not be conflated with self-sufficiency as individuals may require long-term support to live a meaningful life in the community.

The importance of these skills was accentuated when considering the service-user group PAs intended for Woodlands:

"when people have been institutionalised, and I go from the experience of working in the field, and with people who have been (.) erm in (.) institutionalised (.) they expect everything to be done for them (.) they expect someone to do it all for them, so it becomes quite a challenging time then, when you're trying (.) we see it as empowering them, but they don't see it as that, they see it as you're telling me what to do, you're telling me I have to do this, you're telling me I have to do that" (PA Ethel)

Ethel suggested that when people are institutionalised, they hold the expectation that things will be done for them by staff or they lose the skill to do things. The difficulty is that the process of deinstitutionalisation may mean that the population of institutionalised service-users may no longer exist in ways it has done previously. Whilst there is a group of long stay service-users that exists, these individuals are fewer, therefore PAs may be preparing for a non-existent group. Despite this, even a short hospital stay can affect an individual's confidence and mastery of tasks, therefore support to learn, or relearn these skills is still necessary (Owen-Smith et al., 2014).

Ethel noted that the expectations of service-users to complete DLT could be difficult as they could perceive this as a form of control and disempowerment. This suggests that service-users may not have a choice as to whether they complete DLT. This suggests that DLT could be imposed upon service-users, which deviates from another value in their philosophy, choice. The version of recovery that both Elizabeth and Ethel present here may conflate independent living with self-sufficiency, which could be oppressive and disempowering for some service-users.

I have translated the assumptions of how DLT are expected to work at Woodlands into an initial CMOC (see Figure 17) which will later be refined using staff and service-user accounts in which they claim how DLT were operationalised at Woodlands. 


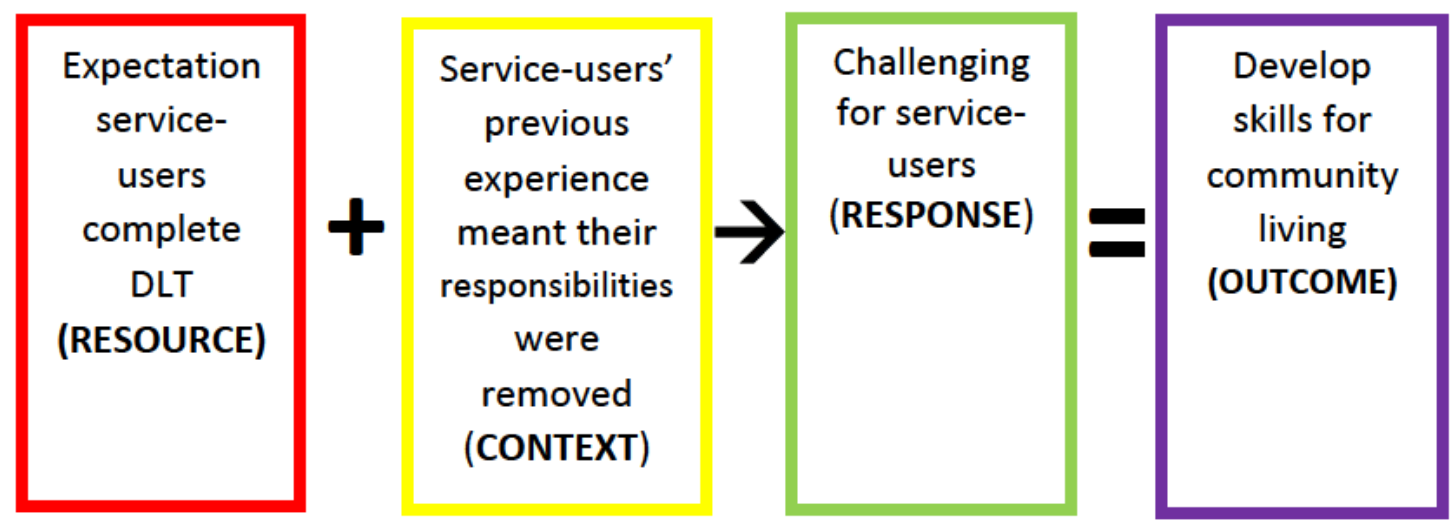

Figure 17 - Initial CMOC for DLT

\subsection{The Reality of Daily Living Tasks}

PAs rationale for DLT was for service-users to develop skills to be able to live independently in the community. I argue that, similarly to what was found in section 6.3 relating to choice, DLT relied upon individual staff interpretation, as opposed to a staff consensus.

Kuly's account suggests the types of DLT service-users were expected to take responsibility for:

"they have to partake in the cooking, they have to partake in the cleaning, they have got to tidy their room, which sounds silly but in higher security services all of it is done for them (.) they've got set times for meals, they don't have to clean for themselves, little things we take for granted that we can all do, they have done for them for years (.) I think it is frightening for them then cause they are thinking well that's not going to be done for me (.) I'm on my own" (Staff Kuly)

Kuly suggests that Woodlands was guided by social norms and expectations of individuals within western societies, such as cooking and cleaning. This suggests that Woodlands have judged what a minimum set of skills are for individual living and are trying to equip and skill individuals so that they are best prepared for what comes after Woodlands.

Kuly indicates two extremes to illustrate the contrast in how DLTs are completed in inpatient services. The first positions staff as responsible for completing all DLT and deprives service-users of responsibility; the second positions service-users as solely responsible for DLT. Kuly's account demonstrates that the gulf between previous 
expectations and those that now hold at Woodlands can be significant. These polarised approaches overlook the reality that service-users are likely to require some form of support to enable them to take on responsibilities they have previously been deprived of with being in inpatient care. This could leave service-users feeling unable to engage, scared and overwhelmed at the prospect of reintroducing and accepting these responsibilities again, and may require more staff support, at least initially, to pick up these skills as part of their new routine.

Kuly's account suggests that service-users felt alone, alluding to them not feeling support was available to assist them in completing DLT, suggesting self-reliance may have been promoted. Deegan (1988) suggested that for some individuals with a mental illness, traditional values such as individual achievement, independence and self-sufficiency are oppressive, and resources built on these values could be invitations for failure. It appears that DLTs have been embedded in a way which promotes self-sufficiency, which could be problematic for some service-users who are unable to meet these expectations. This means that staff need to be flexible in their approaches so that individuals who need more support are provided with it.

Mary suggested that the intended delivery of DLT was adapted due to Woodlands' low service-user occupancy at Woodlands:

"when there is just one person participating in it, it becomes really frustrating for that person cause that is a huge workload that someone is taking on (.) and I think as we get fuller it will get easier, cause say we have a cooking task and a cleaning task to do (.) when there is 3 people here that is quite a lot isn't it? But when there is 16 that is hardly anything at all, so I think it will be easier on guests when we are fuller" (Staff Mary)

Mary's account suggests that the number of DLT service-users were expected to complete depended upon the occupancy at Woodlands; suggesting that service-users contribute to the group effort of ensuring all DLT are completed. This highlights the importance of getting everyone at Woodlands to agree to these community values. If individuals do not contribute, there is a potential for this collectivist approach to breakdown. A group contribution to the completion of DLTs aligns with the collectivist values of recovery 
promoted in service-user defined recovery (Deegan, 1988). However, due to the low occupancy, the collectivist values could not be operationalised in the way intended.

The operationalisation of DLT was also influenced by the realities of being an inpatient service:

"when I first got here I did a lot of the cleaning but now it just pisses me off cause you've got to ask them to get the hoover, out of the cupboard so you can actually hoover it (.) I'm like if you want me to hoover get me the fucking hoover, cause having to ask all the time it does my head in (.) like to do your washing, you have to ask to be let in to do your washing, and then you have to ask to be let into the laundry to take your washing out (.) it's just a pain in the arse" (Service-user Elsie)

The policies and procedures that govern the operation of Woodlands appeared to limit service-users' ability to complete DLT and highlights that the power rested with staff. Additionally, it highlights the responsibility and duty of staff to maintain a safe environment, which appears to be achieved using locked doors. Restricted access to rooms or equipment to complete DLT, was a source of frustration for service-users who had previously engaged. Despite attempts by Woodlands to provide service-users with access to typically restricted items, staff must cope with the frustrations these restrictions cause to them and those accessing the service.

Elsie's account highlights a difference between service-users, and individuals who reside in the community - service-users are restricted, whereas individuals in the community can freely gain access to the resources needed to complete DLT. Deegan $(1988$, p. 7) suggested that practices that reflect the implicit supposition that there is the "world of the abnormal" and the "world of the normal" can create oppressive environments. This appears to be reflected by the perceived unjustness and environmental restrictions which eventually resulted in Elsie stopping the completion of DLT. Although staff at Woodlands are not able to unilaterally change safety rules and guidelines, it does highlight a potential for staff to take the initiative and offer access before service-users ask.

Mary described how one service-user perceived DLT as an attempt by Woodlands to make her conform to societal norms: 
"I mean we have one lady who will be like I don't want to play the game, just to fit in I shouldn't have to do what everyone else does just to conform with society (.) so it's trying to understand it's not playing a game its building skills it's making them, more erm confident and competent I guess for when they do go back out into the community"

(Staff Mary)

Mary's account implies staff attempted to change service-user attitudes towards developing skills they considered essential for community living. There appears to be a set of expectations guiding Woodlands which enforces the status quo and what is considered acceptable in society, however this appears to be well-meaning so that service-users can be part of the community. Whilst it could be argued that this has the potential to dismiss what is acceptable and meaningful to the service-user (Anthony, 1993), staff have a duty to individuals to offer opportunities that may support individuals to keep well and remain part of their communities. It highlights the challenge mental health services face of needing to find the middle ground where service-users are helped to learn the skills to support their recovery, but they also have autonomy.

Mary suggests that service-users will 'go back out into the community'. Her account alludes to Woodlands and service-users as being separate from the community, but that Woodlands is positioned to bridge this gap. This could be an attempt by Woodlands to support service-users to develop the skills needed to recover a life and participate as an equal citizen. It is reasonable to assume that a meaningful life, as accounted for in serviceuser defined recovery (Deegan, 1988; Anthony, 1993), is not lived in the confines of services. It is also reasonable to assume that services see it as their duty to help people to recover a meaningful life, or least develop the skills to aspire to it, which Woodlands is attempting to do.

\subsubsection{Inconsistent Operationalisation of Daily Living Tasks}

There were inconsistencies in how staff delivered DLT at Woodlands, highlighting that the operationalisation was left open to individual staff's interpretations, rather than a consistent team understanding and application. This was a similar finding to how choice was applied by staff (see section 6.3). 
Mary's account suggests that the implementation of DLT varied dependent upon the staffing team:

"it does depend on the staff team that is on (.) cause you will get some people who will do it all, because they are great, but is it always helpful? But then you will get some people who will be like, no they were allocated that task, so that's what they need to do" (Staff Mary)

Mary identified two types of staff, those who completed the DLT themselves, and those who perceived the allocation of DLT as finite and compulsory. Her accounts suggest that the delivery of DLT was not a shared one due to the variations in staff's response to their completion. Although Mary questioned the helpfulness of staff intervening and completing DLT of service-users' behalf, she categorised these members as 'great', alluding to a potential expectation that staff should complete DLT when service-users did not.

According to service-users it was more common for staff to complete DLT:

"Researcher: and is it usually staff or guests who will do the cooking or cleaning Service-user Diana: staff"

"not everyone does their cleaning hardly anyone else helps cook" (Service-user Elsie)

These accounts suggest that staff completed DLT on service-users' behalf, which could be perpetuating the dependency PAs wanted to avoid. This default position may restrict service-users from choosing how they are supported to live an independent life.

Walter proposed that staff's perception of their own abilities influenced the level of encouragement they provided to service-users in completing DLT:

"it also depends on the staff, cause some staff are more enthusiastic about that sort of thing themselves so they can sweep guests along a lot better than others do (.) you know for instance for cooking some staff are not that confident themselves with that, so they are less likely to enthuse the guests" (Staff Walter) 
Staff self-efficacy (Bandura, 1989) appears to be a factor contributing to the variability in how DLT were operationalised. Walter's account suggests there was an assumption that all staff members would have the knowledge, confidence and ability to support serviceusers in completing DLT. A social constructionist theory of learning suggests that learning develops from guided learning which involves sharing and negotiating knowledge within a social interaction (Vygotsky, 1978). Characteristics of a constructivist learning environment include knowledge being shared between the teacher and learner and that the teachers' role is facilitative and guiding. Therefore, assuming staff possess the knowledge and skill to adopt the teacher role, appears to be overestimating some staff's abilities. Walter's account recognises that service-users may require assistance to complete DLT, which aligns with the values underpinning the independent living movement.

There appeared to be a conflation between self-sufficiency and independence, which occasionally left service-users without the assistance needed to complete DLT. Whilst the purpose of DLT appeared to be to promote independence, which is a key principle of recovery and the independent living movement, some service-users suggested that they were left without support to complete DLT. The analysis of the data highlights the challenge Woodlands, like any mental health service, faces when attempting to find the middle ground between autonomy and independence, and offering service-users the support necessary to develop the skills to live independently in the community. I have used the findings from staff and service-users in which they claim how DLT were operationalised in practice refine the initial $\mathrm{CMOC}$ (see Figure 17), to include these findings (see Figure 18). 


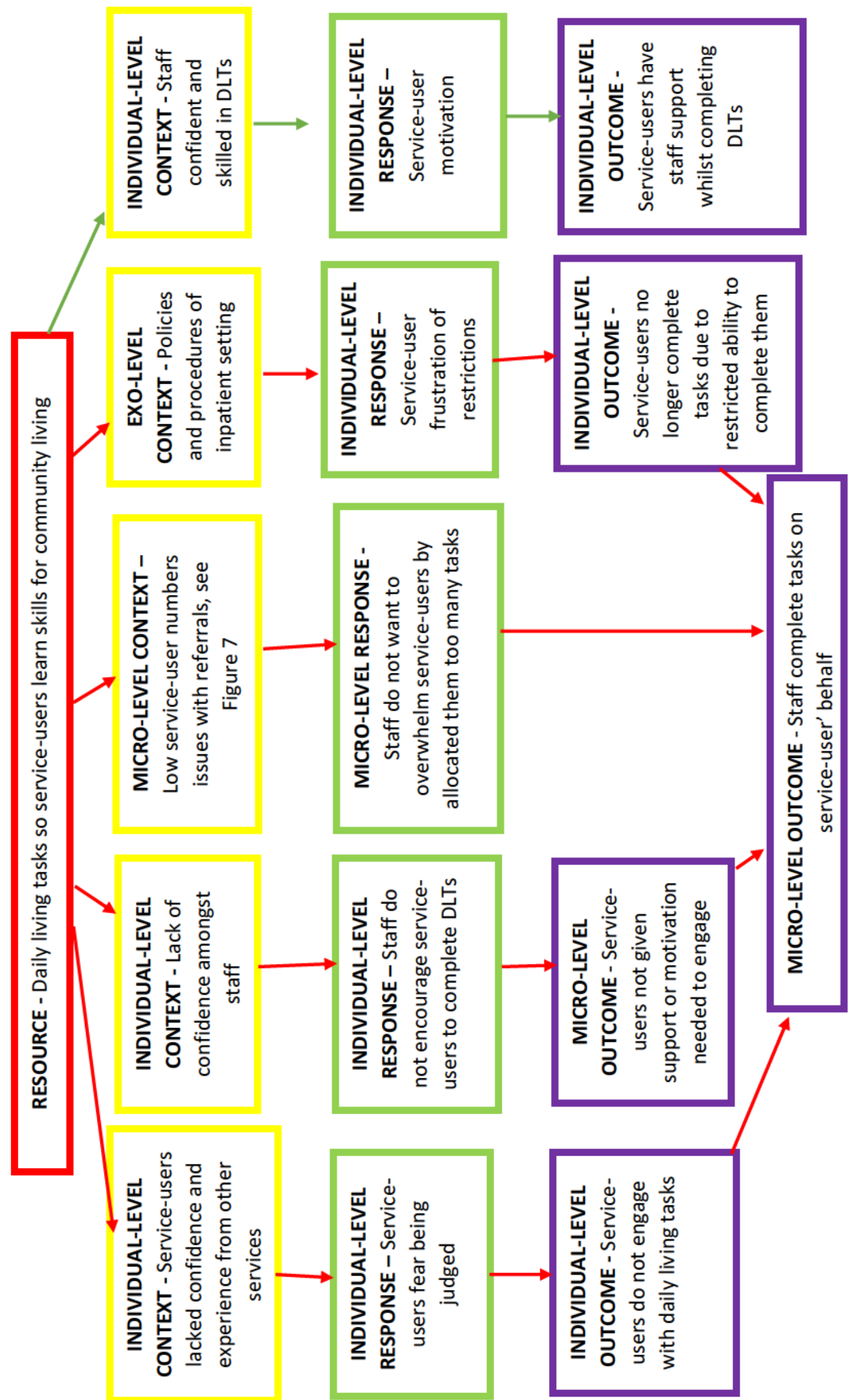

Figure 18 - Revised CMOC for DLT 
Key:

\begin{tabular}{l}
$\longrightarrow \quad$ CMOC - working as intended. \\
$\longrightarrow \quad$ CMOC - not working as intended. \\
\hline
\end{tabular}

\subsection{Summary}

The analysis presented in this chapter highlights that service-user responsibility for their illness and independent living, and their ability to make the 'right' choices were considered important facets of Woodlands approach to recovery. Service-users who were unable to meet the ideals of making choices or taking responsibility, were categorised by some, either implicitly or explicitly, as a poor fit for the service, blamed for this failure, or not provided with the relevant staff support. It could, therefore, be argued that these individuals may have needed extra support to enable them to make the most of the opportunity for choice and responsibility at Woodlands. When this is considered within the context of the excess staff who had the resource of time available to them due to low service-user numbers, it might be reasonable to expect that this extra support would be forthcoming. The reason as to why this extra support was not forthcoming is difficult to discern. In part, from the data available, it appears that the formulation of recovery as having and making choices, being autonomous and taking responsibility seems to have played into a narrative in which service-users are expected to take the initiative, even when they may lack the necessary skills or confidence to do this. This suggests that the staffing group may need to be more assertive, or at least more consistent, in their engagement and support of service-users.

Despite the aspirational intent of PAs and staff to support individuals to make choices and be part of the community, some staff participants claimed that they had to adapt the approach to protect service-users from negative experiences, such as rejection in the wider community. This adaption in Woodlands' model runs the risk of perpetuating societal problems, such as structural violence and ghettoisation. This raises the question of whether a service, like Woodlands, will ever be able to deliver upon their vision of choice, responsibility and service-user participation in the community when stigma, social 
exclusion and social barriers remain within society and prevent service-users from participating as an equal and full citizen in society. Although Woodlands' approach to choice, responsibility and supporting service-users to participate and find a meaningful place within society could be perceived as idealistic, it is also has the potential to cultivate hope and optimism (Slade, 2009). These findings highlight the challenge Woodlands encountered when attempting to support the promotion of choice and the inclusion of service-users within the wider community, and the ongoing, continual battle the service will encounter if there is not a societal shift towards acceptance of individuals with mental ill-health.

\subsubsection{Explanatory Power}

In sum, the following partial, fallible theory suggests that:

- When service-users make choices to engage in community-based opportunities, then there is a risk they may be exposed to societal stigma and rejection;

- When societal change is not within the scope or remit of service delivery, then services will be limited by the extent to which they can support service-users to establish a meaningful and purposeful place within society, which goes against the culture of Woodlands;

- Where service-users have limited experience in making decisions, then staff need to have the ability and skills to be able to adapt their approach so that the support available meets the needs of each individual, therefore not providing a blanket approach to choice or responsibility;

- When there is an inconsistent operationalisation of choice/responsibilities within a service, then there is a high likelihood that service-users do not know what the expectations of them are.

These findings emphasise the importance of ensuring that the appropriate structures are in place so that staff can adjust their approach to meet the needs of the individuals they are working with. Staff at Woodlands appeared to adopt a blanket approach to serviceuser choice and responsibility, which conflicts with the culture and vision of Woodlands 
to provide a recovery model which emphasises the individuality of the recovery journey. Furthermore, there was a tension amongst staff as to where to draw the line between promoting independence and recovery, and failing to provide the appropriate level of support so that the individual could engage with that resource. This suggests again that there was a lack of understanding amongst the workforce as to how to operationalise the culture and vision of Woodlands.

A key explanatory feature of the difficulties in promoting choice and responsibility at Woodlands was the incongruence between ensuring the necessary structures and people were in place to be able to flexibly deliver the recovery approach and culture of Woodlands to meet the needs of the individual. This meant staff were unable to fulfil their work of promoting choice and responsibility for completing DLT in a way which met the needs of the individual, and instead a blanket rule was implemented which did not align with the recovery culture of Woodlands. The findings in this chapter highlight the importance of ensuring that the 'structure' and 'people' components of the model are congruent with the culture of Woodlands which was to provide individualised, ROC (see Figure 8).

In the next chapter, I will present my analysis of the data that was routinely collected at Woodlands. 
7 FINDINGS - QUANTITATIVE CASE STUdIES 


\subsection{Introduction}

In this chapter, I present my analysis of the routinely collected data from Woodlands that was made available to me. I present six clinical case studies to show service-user's journeys and highlight any changes during admission. I highlight the trends that appear across the dataset and will position this alongside current literature in the discussion chapter (see section 8.4).

When conducting field research, the unexpected can arise necessitating flexibility on the part of the researcher. For example, Woodlands agreed for the routine data that they collected as part of their protocol to be anonymised and shared for the purposes of this evaluation. Despite this agreement, only minimal data was recorded and available for analysis. Routinely collected data is defined as data collected for purposes other than research (Safran, 1991), such as health records, observation data and administrative data. It has been suggested that this form of data collection can pose some challenges. For example, some studies suggest that hospital data collection practices are less than systematic, as there is often missing data (Chen et al., 2019); staff perceive this as an additional burden to collect and enter data into a system and may believe that the data collection is unnecessary and therefore do not see the utility of collecting it (Chin et al., 2008); and lastly, the data lacks completeness and accuracy (Jordan et al., 2004). This highlights that the challenges of routinely collected data within healthcare are not unique to Woodlands.

The available data related to 6 service-users. To put this into context Woodlands accepted a total of 14 service-users since opening in 2017 until the end of the data collection period in December 2019. Therefore, the available data accounted for $43 \%$ of the service-users who had accessed Woodlands. Due to the small sample of data available, I will present descriptive statistics derived from the standardised measures collected as routine data and will identify potential trends across the data; however, I will draw no firm inferences. Despite having an agreed protocol (see section 2.6.2.1), there were very few measures collected upon admission, which meant a baseline could not be determined for each service-user; and there was limited follow-up data which meant monitoring changes over time was impacted. 


\section{The ReQoL-10}

Data was collected to examine the service-user's perspective of their recovery and quality of life (QoL) using the 10-item ReQoL-10 (Keetharuth et al., 2017). This was a selfcompleted measure, although service-users could be provided with support to complete the measure if necessary. It was not documented whether staff support was given within the database provided to me. I had access to 9 completed ReQoL-10 measures from 5 service-users. Two service-users completed the ReQoL-10 on one occasion, two serviceusers completed it twice, and one service-user completed it three times. To help me understand how to use these scores I contacted the author of the measure to determine whether there were any cut-offs or categorisations of what the numerical values meant. I was informed that the scores were to be used to determine if there had been a reliable change to individual's QoL over time. If there was a change of 5 or more to the serviceusers total score, this denoted a reliable deterioration or improvement in QoL, meaning the change was not due to error or chance. The ReQoL-10 total score can range from 040 with 0 indicating the poorest QoL and 40 indicating the highest. In the current study, the total scores ranged from 12-34, with a mean of 22.5 .

\section{CORE-OM}

The CORE-OM is a 34-item administered measure used to assess the efficacy and effectiveness of therapeutic input concerning service-user distress (Evans et al., 2002). I had access to 7 completed CORE-OM measures from 3 service-users. One service-user completed the measure once and two completed it three times. The total score was calculated by summing up all the items, dividing by 34 (number of items) and x10 to provide a number on the 10-40 scale. A higher score indicated a higher psychological distress. The clinical cut-off point when using the 10-40 scale was 10 . The reliable change index $(\mathrm{RCI})$ for the CORE-OM is 5 , which denotes a reliable change that was greater than chance or measurement error. The following grading was used to denote the severity of a service-users psychological distress, using the 10-40 scale (see Table 6) and will be used within the clinical case studies. In the current study, the total scores ranged from 11.824.7, with a mean of 18.5 . 


\begin{tabular}{|l|c|}
\hline $\begin{array}{c}\text { Severity of Psychological } \\
\text { Distress }\end{array}$ & Score \\
\hline Severe & $25-40$ \\
\hline Moderate Severe & $20-25$ \\
\hline Moderate & $15-20$ \\
\hline Mild & $10-15$ \\
\hline Low Level & $5-10$ \\
\hline Healthy & $0-5$ \\
\hline
\end{tabular}

Table 6 - CORE-OM Scores relating to Severity of Service-User Psychological Distress

\section{VOICE}

Data was collected to examine service-user's perspectives of therapeutic contact, care, trust and respect they received from staff using the VOICE (Evans et al., 2012). This was a self-completed measure, although service-users were provided with support if necessary, although it was not documented whether support was given within the database provided to me. I had access to 8 completed VOICE measures from 5 service-users. Three serviceusers completed the VOICE once, one service-user completed it twice and one serviceuser on three occasions. I contacted the author of the measure for clarification about use of scores and learned that there were no cut-offs or categorisations to contextualise the numerical value of the measure, which is a notable limitation. Although I could have formulated a means of quantifying how to handle these scores into some form of scale, due to volume of data available I did not think this would meaningfully contribute to the results and therefore opted to not specifically focus on the numerical value of this measure. The VOICE total score can range from 19-114; with higher scores indicating a more negative perception of inpatient care. In the current study, VOICE scores ranged from 30-46, with a mean of 39. It is important to note that the VOICE measure contained spaces for 'Any Comments'; however, this data was not completed by any service-user within the current sample. Therefore, I was unable to use this additional data to begin to understand why service-users may have rated items in the way they did. It was, therefore, important that I kept this limitation in mind when discussing the relevance and possible meaning of the items and potential trends when presenting the clinical case studies. 
I now present my analysis of the data in the form of six clinical case studies. Each measure will be discussed separately, but due to the similarity of some of the items across the three measures, there will be occasions where I will discuss the measures together to avoid repetition. 


\subsection{Shara}

Shara was admitted in the summer of 2017 and at the end of data collection had been at Woodlands for 2 years 6 months. The data available for Shara consisted of three ReQoL10 measures, three VOICE measures and three CORE-OM measures.

\subsubsection{ReQoL-10}

Firstly, I noted that there were no ReQoL-10 measures collected until 2019, which was 2 years after Shara's admission. The available ReQoL-10 data for Shara showed there was a deterioration over time of 10 points to her QoL (see Table 7), denoting a reliable deterioration to her QoL that cannot be attributed to chance or error.

\begin{tabular}{|l|c|c|c|}
\hline \multicolumn{1}{|c|}{ ReQoL-10 } & March 2019 & April 2019 & $\begin{array}{c}\text { September } \\
\mathbf{2 0 1 9}\end{array}$ \\
\hline $\begin{array}{l}\text { I found it difficult to get started } \\
\text { with everyday tasks }\end{array}$ & Sometimes & Often & Sometimes \\
\hline I felt able to trust others & Occasionally & Occasionally & Occasionally \\
\hline I felt unable to cope & Occasionally & Sometimes & Occasionally \\
\hline $\begin{array}{l}\text { I could do the things I wanted to } \\
\text { do }\end{array}$ & Often & Sometimes & Often \\
\hline I felt happy & Often & Sometimes & Occasionally \\
\hline $\begin{array}{l}\text { I thought my life was not worth } \\
\text { living }\end{array}$ & Occasionally & Often & Often \\
\hline I enjoyed what I did & Often & Sometimes & Sometimes \\
\hline I felt hopeful about my future & Often & Occasionally & Occasionally \\
\hline I felt lonely & Occasionally & Often & Often \\
\hline I felt confident in myself & Sometimes & Occasionally & Occasionally \\
\hline $\begin{array}{l}\text { Physical health problems } \\
\text { Moderate }\end{array}$ & $\begin{array}{c}\text { No Problems } \\
\text { Problems }\end{array}$ & $\begin{array}{c}\text { Severe } \\
\text { Problems }\end{array}$ \\
\hline Total Score & $\mathbf{2 8}$ & $\mathbf{1 8}$ & $\mathbf{1 9}$ \\
\hline
\end{tabular}

Table 7 - Shara's ReQoL-10 Data

Several ReQoL-10 items appeared to be contributing to the decline in Shara's QoL. I identified any item where there had been an improvement, no improvement or a decline 
between measures. However, I am unable to determine the reasons as to why Shara rated these items in this way, therefore, caution in interpreting these results was used throughout.

Despite there being an overall deterioration in Shara's QoL, there was one item she rated positively which related to doing the things she wanted to do. This may suggest that Shara did not feel restricted in what she was able to do whilst at Woodlands.

The ReQoL-10 data suggested Shara experienced increased loneliness, highlighting a discrepancy between the relationships Shara had and the relationships she wanted. This was supported with her CORE-OM data which indicated that Shara felt more alone, isolated, did not always have support when needed and had no friends (see Table 7). This could suggest that there may be differences between availability of social support, as measured by proximity, and the supply of social support in the form of close, or intimate, relationships. It may also say something about the differences between how service-users engage with staff, versus others, such as friendships. The data shows that Shara's loneliness score dropped within one month, and then remained low when measured again 6 months later. As there was no data available for her admission point, it is difficult to compare whether there had been a deterioration in loneliness since being at Woodlands, or whether this was a residual difficulty. Despite her loneliness, Shara's COREOM data did suggest that she felt some warmth or affection for someone, which suggests she experienced some form of connection with someone. This may say something about Shara's need for more than one connection, as despite feeling warmth or affection, she still rated that she had a lack of social support.

Another trend related to Shara thinking that her life was not worth living, feeling hopeless for the future and feeling unhappy (see Table 7). Her CORE-OM data also suggested there was an escalation in Shara's suicidal ideation, including thinking she would be better off dead, engaging in physically harmful and risky behaviours towards herself and her health, and having a suicide plan (see Table 8).

Shara's physical health initially improved and then worsened (see Table 7). However, her CORE-OM data, suggested there was a reduction in Shara being troubled by aches, pains or other physical problems (see Table 8). This could suggest that although Shara's physical 
health problems were still present, the impact of these lessened over time; suggesting she may have developed her ability to cope and live with the effects of her physical health, or she was less concerned about these problems.

There were no improvements in Shara's trust towards others (see Table 7). It should be noted that this item does not focus specifically upon trust with staff, but I consider this finding within the context of staff and other forms of support. The lack of trust suggests that any attempts aimed at developing trust between Shara and staff were insufficient or may not have occurred. Alternatively, as Shara did not feel she had friends, and often felt alone, see may feel that she does not have a social support network in which she can place her trust and therefore opportunities for her to trust others are limited.

\subsubsection{CORE-OM}

The first CORE-OM available for Shara was completed 9 months into her admission, therefore determining changes compared to admission was not possible. Shara's COREOM total score shows that there was an improvement in her rated psychological distress between March and June 2018, but that this worsened by September 2019, where her psychological distress was categorised as severe (see Table 8).

As I have already discussed some of the CORE-OM data alongside the ReQoL-10 data I will only refer to relevant items that have not been discussed elsewhere. These items may be associated with the deterioration in Shara's rated psychological distress, however I am unable to determine from the data why this deterioration occurred, or why she rated these items in this way. Despite these limitations, I will consider potential trends, exercising caution in interpretation throughout.

\begin{tabular}{|l|c|c|c|}
\hline \multicolumn{1}{|c|}{ CORE-OM } & $\begin{array}{c}\text { March } \\
\mathbf{2 0 1 8}\end{array}$ & $\begin{array}{c}\text { June } \\
\mathbf{2 0 1 8}\end{array}$ & $\begin{array}{c}\text { September } \\
\mathbf{2 0 1 9}\end{array}$ \\
\hline I have felt terribly alone and isolated & Sometimes & Sometimes & Often \\
\hline I have felt tense, anxious or nervous & $\begin{array}{c}\text { Most/All } \\
\text { the Time }\end{array}$ & $\begin{array}{c}\text { Most/All } \\
\text { the Time }\end{array}$ & $\begin{array}{c}\text { Most/All } \\
\text { the Time }\end{array}$ \\
\hline $\begin{array}{l}\text { I have felt I have someone to turn to } \\
\text { for support when needed }\end{array}$ & Often & Often & Sometimes \\
\hline
\end{tabular}




\begin{tabular}{|c|c|c|c|}
\hline I have felt O.K. about myself & Occasionally & Occasionally & Occasionally \\
\hline $\begin{array}{l}\text { I have felt totally lacking in energy } \\
\text { and enthusiasm }\end{array}$ & Occasionally & Often & Often \\
\hline $\begin{array}{l}\text { I have been physically violent to } \\
\text { others }\end{array}$ & Not at all & Not at all & Not at all \\
\hline $\begin{array}{l}\text { I have felt able to cope when things } \\
\text { go wrong }\end{array}$ & Sometimes & Often & Occasionally \\
\hline $\begin{array}{l}\text { I have been troubled by aches, pains } \\
\text { or other physical problems }\end{array}$ & Often & Occasionally & Not at all \\
\hline I have thought of hurting myself & Often & Often & $\begin{array}{l}\text { Most/All } \\
\text { the time }\end{array}$ \\
\hline $\begin{array}{l}\text { Talking to people has felt too much } \\
\text { for me }\end{array}$ & Sometimes & Sometimes & Sometimes \\
\hline $\begin{array}{l}\text { Tension and anxiety have prevented } \\
\text { me doing important things }\end{array}$ & Occasionally & Not at all & Sometimes \\
\hline $\begin{array}{l}\text { I have been happy with the things I } \\
\text { have done }\end{array}$ & Occasionally & Often & Occasionally \\
\hline $\begin{array}{l}\text { I have been disturbed by unwanted } \\
\text { thoughts and feelings }\end{array}$ & Often & Often & $\begin{array}{l}\text { Most/All } \\
\text { the time }\end{array}$ \\
\hline I have felt like crying & Often & Sometimes & Often \\
\hline I have felt panic or terror & Sometimes & Sometimes & $\begin{array}{l}\text { Most/All } \\
\text { the time }\end{array}$ \\
\hline I made plans to end my life & Occasionally & Often & Often \\
\hline $\begin{array}{l}\text { I have felt overwhelmed by my } \\
\text { problems }\end{array}$ & Sometimes & Sometimes & $\begin{array}{l}\text { Most/All } \\
\text { the time }\end{array}$ \\
\hline $\begin{array}{l}\text { I have had difficulty getting to sleep } \\
\text { or staying asleep }\end{array}$ & Occasionally & Occasionally & Sometimes \\
\hline $\begin{array}{l}\text { I have felt warmth or affection for } \\
\text { someone }\end{array}$ & Often & Often & Often \\
\hline $\begin{array}{l}\text { My problems have been impossible } \\
\text { to put to one side }\end{array}$ & Sometimes & Occasionally & Often \\
\hline $\begin{array}{l}\text { I have been able to do most things I } \\
\text { needed to }\end{array}$ & Often & $\begin{array}{l}\text { Most/All } \\
\text { the time }\end{array}$ & Sometimes \\
\hline
\end{tabular}




\begin{tabular}{|c|c|c|c|}
\hline $\begin{array}{l}\text { I have threatened or intimidated } \\
\text { another person }\end{array}$ & Not at all & Not at all & Not at all \\
\hline I have felt despairing or hopeless & Often & Sometimes & Often \\
\hline $\begin{array}{l}\text { I have thought it would be better if I } \\
\text { were dead }\end{array}$ & Often & Sometimes & Often \\
\hline I have felt criticised by other people & Not at all & Occasionally & Occasionally \\
\hline I have thought I have no friends & Often & Sometimes & Often \\
\hline I have felt unhappy & Often & Sometimes & Often \\
\hline $\begin{array}{l}\text { Unwanted images or memories have } \\
\text { been distressing me }\end{array}$ & Often & Often & Often \\
\hline $\begin{array}{l}\text { I have been irritable when with other } \\
\text { people }\end{array}$ & Sometimes & Sometimes & Often \\
\hline $\begin{array}{l}\text { I have thought I am to blame for my } \\
\text { problems and difficulties }\end{array}$ & Often & Sometimes & Often \\
\hline I have felt optimistic about my future & Occasionally & Occasionally & Occasionally \\
\hline $\begin{array}{l}\text { I have achieved the things I wanted } \\
\text { to }\end{array}$ & Sometimes & $\begin{array}{l}\text { Most/All } \\
\text { the time }\end{array}$ & Occasionally \\
\hline $\begin{array}{l}\text { I have felt humiliated or shamed by } \\
\text { other people }\end{array}$ & Not at all & Not at all & Not at all \\
\hline $\begin{array}{l}\text { I have hurt myself physically or taken } \\
\text { dangerous risks with my health }\end{array}$ & Not at all & Not at all & Often \\
\hline Total Score & $\begin{array}{c}20 \\
\text { (Moderate } \\
\text { Severe) }\end{array}$ & $\begin{array}{c}15.6 \\
\text { (Moderate) }\end{array}$ & $\begin{array}{c}24.7 \\
\text { (Severe) }\end{array}$ \\
\hline
\end{tabular}

Table 8 - Shara's CORE-OM Data

Shara's CORE-OM data suggested that she would often lack energy and enthusiasm. Shara's score at 9 months indicated that she did not feel she had these. Shara's difficulties with energy and enthusiasm could be due to several reasons, such as residual symptoms of her mental illness or side-effects of her medication; however, I am not able to determine the reasons underlying this from the data available. It does appear that a lack of energy and enthusiasm was a residual difficulty for Shara. 
Lastly, there were no improvement in Shara's optimism for the future. Her rating for optimism aligned with the hopelessness from her ReQoL-10 data (see Table 7). This could be due to the fact recovery is not a linear process and as hope is considered a turning point in someone's recovery (Deegan, 1988), these ratings may therefore indicate where Shara was in her recovery.

\subsubsection{VOICE}

The VOICE data available for Shara highlighted that there was a decline in her scores concerning her perception of Woodlands. There was an increase of 1 in Shara's VOICE total score from September 2018 to April 2019, however there was an increase of 12 from April 2019 to September 2019 (see Table 9). Although I am not able to deduce whether this was a consistent decline in Shara's ratings of Woodlands, or whether this represented a one-off month, there does appear to be a potential trend alluding to a deterioration in her perception of Woodlands over time.

\begin{tabular}{|l|c|c|c|}
\hline \multicolumn{1}{|c|}{ VOICE } & Sep-18 & Apr-19 & Sep-19 \\
\hline $\begin{array}{l}\text { Made to feel welcome on } \\
\text { arrival }\end{array}$ & Strongly Agree & Strongly Agree & Strongly Agree \\
\hline $\begin{array}{l}\text { I have a say in my care and } \\
\text { treatment }\end{array}$ & Strongly Agree & Strongly Agree & Agree \\
\hline $\begin{array}{l}\text { Ward rounds are useful for } \\
\text { me }\end{array}$ & Strongly Agree & Strongly Agree & Strongly Agree \\
\hline $\begin{array}{l}\text { I feel medication helps me } \\
\text { I have an opportunity to } \\
\text { discuss my medication and } \\
\text { side effects }\end{array}$ & $\begin{array}{c}\text { Slightly } \\
\text { Disagree }\end{array}$ & Slightly Agree & $\begin{array}{c}\text { Slightly } \\
\text { Disagree }\end{array}$ \\
\hline $\begin{array}{l}\text { Staff give me medication } \\
\text { instead of talking to me }\end{array}$ & Disagree & Disagree & Strongly Agree \\
\hline $\begin{array}{l}\text { Staff take an interest in me } \\
\text { Disagree }\end{array}$ \\
\hline $\begin{array}{l}\text { Staff are available to talk } \\
\text { when I need them }\end{array}$ & Strongly Agree & Strongly Agree & Agree \\
\hline $\begin{array}{l}\text { I trust the staff to do a good } \\
\text { job }\end{array}$ & Strongly Agree & Strongly Agree & Agree \\
\hline
\end{tabular}




\begin{tabular}{|c|c|c|c|}
\hline $\begin{array}{l}\text { I feel that staff understand } \\
\text { how my illness affects me }\end{array}$ & Agree & Slightly Agree & $\begin{array}{l}\text { Slightly } \\
\text { Disagree }\end{array}$ \\
\hline $\begin{array}{l}\text { I feel staff treat me with } \\
\text { respect }\end{array}$ & Strongly Agree & Strongly Agree & Strongly Agree \\
\hline $\begin{array}{l}\text { I think the activities on the } \\
\text { ward meet my needs }\end{array}$ & Slightly Agree & Slightly Agree & $\begin{array}{l}\text { Slightly } \\
\text { Disagree }\end{array}$ \\
\hline $\begin{array}{l}\text { I find the one-to-one time } \\
\text { with staff useful }\end{array}$ & Strongly Agree & Strongly Agree & Strongly Agree \\
\hline $\begin{array}{l}\text { I find it easy to keep in } \\
\text { contact with family while I'm } \\
\text { on the ward }\end{array}$ & Strongly Agree & Strongly Agree & Strongly Agree \\
\hline I feel safe on the ward & Strongly Agree & Strongly Agree & Strongly Agree \\
\hline $\begin{array}{l}\text { I feel staff respond well when } \\
\text { the panic alarm goes off }\end{array}$ & Agree & Slightly Agree & Strongly Agree \\
\hline $\begin{array}{l}\text { I feel staff respond well when } \\
\text { I tell them I'm in crisis }\end{array}$ & Agree & Slightly Agree & $\begin{array}{l}\text { Slightly } \\
\text { Disagree }\end{array}$ \\
\hline $\begin{array}{l}\text { I feel able to practice my } \\
\text { religion whilst I'm in hospital }\end{array}$ & Strongly Agree & Strongly Agree & Agree \\
\hline $\begin{array}{l}\text { I think staff respect my ethnic } \\
\text { background }\end{array}$ & Strongly Agree & Strongly Agree & Agree \\
\hline Total Score & 32 & 33 & 45 \\
\hline
\end{tabular}

Table 9 - Shara's VOICE Data

There were items which Shara rated more negative, positively, or did not improve which influenced her total score which I will now discuss.

There were VOICE items that Shara rated positively. Shara rated the ease of remaining in contact with her family favourably, suggesting that there were structures in place to enable the maintenance of this support network. Positively rated items also included the usefulness of ward rounds, her one-to-ones with staff and staff not giving her medication rather than talking to her. Across her VOICE data, Shara appears to value most of her interactions with staff, which fits with many studies that show service-users value this element of their care above most (Coffey et al., 2019a). However, one item in which Shara's rating decreased related to staff's availability to talk to when needed. This potential trend is of interest as Shara rated that her one-to-ones with staff were useful, 
suggesting she valued these interactions, however, the frequency and ease of accessing this support did not appear to meet her needs.

One item that Shara rated as "slightly disagree" was that the activities available on the ward were not meeting her needs (see Table 9). Something worth noting about this specific item is the terminology used. The term 'ward' is not used to describe Woodlands and therefore this may be confusing for service-users completing the VOICE measure and not considered applicable in their circumstance.

Another item Shara rated less favourably related to how staff responded to her when she was in crisis (see Table 9). Whilst it is noted that Shara's and staff's perception of crisis management may not align, Woodlands' crisis approach does not appear to have been what Shara wanted. This appears to diverge with other items in the VOICE measure which suggested Shara felt safe, confident in staff and respected by them. This may suggest that Shara's perception of staff support altered when she was in crisis, however when she was not in crisis, she has a better experience of her relationship with staff. If this is the case, then this reflects a challenge, that both Shara and staff may face, in maintaining a therapeutic relationship that may be in flux.

Shara did not feel that the staff understood how her mental illness affected her, which suggests there may have been some challenges to Shara and staff establishing a relationship in which these conversations could happen. Alternatively, these conversations may not have occurred due to the lack of trust Shara felt towards others (see Table 7). This may suggest that the development of a therapeutic relationship between Shara and staff experienced some challenges, which prevented key conversations around crisis management and her mental illness.

\subsection{Maddie}

Maddie was admitted in the Spring of 2019 and at the end of the data collection period had been at Woodlands for 9 months. Maddie completed the ReQoL-10 measure twice and the VOICE measure once. There was no CORE-OM data available for Maddie. 


\subsubsection{ReQoL-10}

There was no ReQoL-10 data available for Maddie upon admission, but the first measure was completed one month into her placement and the second was completed 8 months later (see Table 10). There appeared to be a deterioration in Maddie's QoL between these two data points.

\begin{tabular}{|l|c|c|}
\hline \multicolumn{1}{|c|}{ ReQoL-10 } & April $\mathbf{2 0 1 9}$ & December $\mathbf{2 0 1 9}$ \\
\hline $\begin{array}{l}\text { I found it difficult to get started } \\
\text { with everyday tasks }\end{array}$ & Sometimes & Most/All the time \\
\hline I felt able to trust others & None of the time & None of the Time \\
\hline I felt unable to cope & Most/All the time & Most/All the time \\
\hline $\begin{array}{l}\text { I could do the things I wanted to } \\
\text { do }\end{array}$ & Sometimes & None of the time \\
\hline I felt happy & None of the time & Often \\
\hline $\begin{array}{l}\text { I thought my life was not worth } \\
\text { living }\end{array}$ & Most/All the time & Most/All the time \\
\hline I enjoyed what I did & Most/All the time & Sometimes \\
\hline I felt hopeful about my future & Sometimes & Occasionally \\
\hline I felt lonely & Most/All the time & Most/All the time \\
\hline I felt confident in myself & None of the time & None of the Time \\
\hline Physical health problems & Moderate Problems & Moderate Problems \\
\hline Total Score & $\mathbf{1 2}$ & $\mathbf{8}$ \\
\hline
\end{tabular}

Table 10 - Maddie's ReQoL-10 Data

There were some areas within the ReQoL-10 which Maddie rated lower, or consistently the same which appeared to be contributing to the deterioration in her QoL. I will now consider these items in turn.

Firstly, there was a decrease in Maddie's ability to get started with everyday tasks, this shows that she rated this as an issue and that it got worse over time. This suggests that this was a residual difficulty that may require additional support from staff. There was also a decrease in how Maddie rated being able to do the things she wanted to and her enjoyment of these activities. Whilst the item does not measure whether Maddie engaged 
in activities of her choosing, only her perception, it does suggest a worsening during her admission.

There were several ReQoL-10 items in which there were no changes in Maddie's rating. Maddie's coping did not change, which suggests that this remains a residual area of difficulty. There were no changes to Maddie thinking that her life was worth living, which aligns with her hopelessness for the future. Maddie's hopelessness may, however, represent where she is in her recovery journey. Maddie's rated loneliness did not change suggesting there was a discrepancy between her actual relationships, and what she wanted from her relationships. Lastly, there were no changes to Maddie's physical health problems. The quantitative data available does not provide an understanding as to why these areas did not improve, however, it does suggest that these areas remain residual difficulties for Maddie in which she may need additional support to address.

There was one item in Maddie's CORE-OM data that improved over time, her happiness, suggesting that she felt contentment at the time of completing the measure.

\subsubsection{VOICE}

Maddie completed the VOICE on one occasion; therefore, I was not able to consider any changes to her rated perception of Woodlands over time (see Table 11). There were, however, certain items that appeared relevant to how she perceived Woodlands, which I will now discuss.

\begin{tabular}{|l|c|}
\hline \multicolumn{1}{|c|}{ VOICE } & April $\mathbf{2 0 1 9}$ \\
\hline Made to feel welcome on arrival & Agree \\
\hline I have a say in my care and treatment & Strongly Disagree \\
\hline Ward rounds are useful for me & Slightly Agree \\
\hline I feel medication helps me & Strongly Disagree \\
\hline I have an opportunity to discuss my medication and side effects & Agree \\
\hline Staff give me medication instead of talking to me & Strongly Disagree \\
\hline Staff take an interest in me & Agree \\
\hline Staff are available to talk when I need them & Agree \\
\hline I trust the staff to do a good job & Slightly Agree \\
\hline I feel that staff understand how my illness affects me & Strongly Disagree \\
\hline I feel staff treat me with respect & Slightly Agree \\
\hline I think the activities on the ward meet my needs & Agree \\
\hline
\end{tabular}




\begin{tabular}{|l|c|}
\hline I find the one-to-one time with staff useful & Strongly Agree \\
\hline $\begin{array}{l}\text { I find it easy to keep in contact with family while I'm on the } \\
\text { ward }\end{array}$ & Strongly Agree \\
\hline I feel safe on the ward & Strongly Disagree \\
\hline I feel staff respond well when the panic alarm goes off & Strongly Agree \\
\hline I feel staff respond well when I tell them I'm in crisis & Slightly Agree \\
\hline I feel able to practice my religion whilst I'm in hospital & Slightly Agree \\
\hline I think staff respect my ethnic background & Slightly Agree \\
\hline Total Score & $\mathbf{6 1}$ \\
\hline
\end{tabular}

Table 11 - Maddie's VOICE Data

Maddie's responses were favourable for items that relate to the usefulness of one-to-one time with staff, her ability to keep in contact with her family and staff's response to when the panic alarm goes. This suggests that Maddie did value her interactions with staff. Given the fact this measure was completed close to her admission this could allude to the early signs of a therapeutic relationship forming between Maddie and the staff. However, due to no follow-up data being available for Maddie, I am unable to see whether these responses changed over time. Maddie also rated that she was able to maintain contact with her family, which suggests that there were structures in place so that Maddie did not lose her support network outside of Woodlands.

Maddie's responses suggest that she did not feel she could have a say in her care and treatment. It should be noted that this measure was taken close to Maddie's admission and therefore this rating could be attributed to staff affording Maddie time to adjust to being at Woodlands and to establish relationships with staff before engaging her in conversations regarding her treatment.

Maddie's responses suggest that she did not feel the staff understood how her illness affected her. Due to the limited time Maddie had been at Woodlands when she completed the VOICE, Maddie may not have formed relationships with staff this early into her admission, which could have limited these types of conversations from occurring. However, this cannot be determined given the lack of follow-up data available. 


\subsection{Jess}

Jess was admitted in the Winter of 2018, which meant she had been at Woodlands for 1 year at the end of data collection. The data available for Jess consisted of one ReQoL-10 measure and one VOICE measure which were both completed 4 months and three COREOM measures, which were completed one month, two months and three months into Jess' admission.

\subsubsection{ReQoL-10}

Jess' ReQoL-10 data was limited as there was no admission data available to establish a baseline score, nor was there any follow-up data which restricted my ability to determine any changes over time (see Table 12).

\begin{tabular}{|l|c|}
\hline \multicolumn{1}{|c|}{ ReQoL-10 } & April $\mathbf{2 0 1 9}$ \\
\hline I found it difficult to get started with everyday tasks & Sometimes \\
\hline I felt able to trust others & None of the time \\
\hline I felt unable to cope & Occasionally \\
\hline I could do the things I wanted to do & Most/All the time \\
\hline I felt happy & Occasionally \\
\hline I thought my life was not worth living & Sometimes \\
\hline I enjoyed what I did & Most/All the time \\
\hline I felt hopeful about my future & Most/All the time \\
\hline I felt lonely & Most/All the time \\
\hline I felt confident in myself & Sometimes \\
\hline Physical health problems & Severe Problems \\
\hline Total Score & $\mathbf{2 1}$ \\
\hline
\end{tabular}

Table 12 - Jess' ReQoL-10 Data

Jess' rating of the ReQoL-10 items show areas which appeared to be positively or negatively contributing to her QoL at the time of completing the measure. I will now discuss these items separately.

There were items within the ReQoL-10 that Jess rated favourably. Jess felt that she was able to do the things that she wanted to do. Jess rated that she enjoyed the things that she did; which suggests she derived some form of pleasure from what she did. However, 
this was inconsistent with Jess' CORE-OM which suggested that she did not enjoy what she did (see Table 13). This highlights that Jess' enjoyment of what she did varied at different time points of her admission, which is unsurprising as enjoyment is unlikely to be consistent and could be contingent upon several factors, such as how Jess felt on the day she completed the measure.

Another item that Jess rated favourably was her hopefulness for the future. However, Jess' CORE-OM suggested that she felt hopeless, despairing and lacked optimism for the future. This highlights that Jess was hopeful at times, but this sense of hope fluctuated and was not maintained. This is to be expected as recovery is not a linear process, and may involve setbacks, or fluctuations in hope, and therefore, the variability in how Jess has rated hope-specific items may reflect her recovery journey.

The first item Jess rated less favourably related to loneliness, which was also seen in her ratings of the CORE-OM data (see Table 13). This suggests there was a discrepancy between Jess' desired and perceived social relationships. However, Jess' CORE-OM data did suggest that there were improvements in feeling she had someone to turn to when needed, felt warmth and affection for someone, and that she had friends. This may suggest that she had relationships in the form of friendship, or someone she could rely upon, but was lacking other forms of relationships which were important to her.

Jess rated that she did not feel able to trust others, which could have hindered her ability to form connections and relationships with others and correlated with her ratings of loneliness. As I only had data from Jess' ReQoL-10 measure for one time point, I was unable to see whether Jess' trust developed and evolved over time. It does, however, highlight a potential challenge that Jess and staff face in being able to develop a relationship if there is a lack of trust.

Jess was experiencing severe physical health problems. Jess' CORE-OM data suggested that there was no progress in how troubled she was by her physical problems, which suggests that her physical health remained an area of residual difficulty and highlights Jess may need support that addresses both her physical and mental health. 


\subsubsection{CORE-OM}

The data available for Jess' CORE-OM was the only measure that was completed in three consecutive months and was first completed relatively close to her admission. Jess' COREOM total score increased by 9.7, between January 2019 and February 2019. This is an increase of more than 5 , denoting a reliable change in her psychological distress that cannot be attributed to chance or error. Jess' CORE-OM total score for March 2019 was 18.8, which shows a decrease in psychological distress of 2.7 (see Table 13). Jess' psychological distress was rated as moderate (January 2019), then moderate severe (February 2019), and then moderate (March 2019).

I now consider the items that show a change, or no improvement in Jess' ratings. As I have already considered some items from the CORE-OM, I only discuss the relevant items that have not been mentioned elsewhere.

\begin{tabular}{|l|c|c|c|}
\hline \multicolumn{1}{|c|}{ CORE-OM } & $\begin{array}{c}\text { January } \\
\mathbf{2 0 1 9}\end{array}$ & $\begin{array}{c}\text { February } \\
\mathbf{2 0 1 9}\end{array}$ & $\begin{array}{c}\text { March } \\
\mathbf{2 0 1 9}\end{array}$ \\
\hline I have felt terribly alone and isolated & Not at all & $\begin{array}{c}\text { Most/All } \\
\text { the time }\end{array}$ & Sometimes \\
\hline I have felt tense, anxious or nervous & Not at all & Occasionally & Not at all \\
\hline $\begin{array}{l}\text { I have felt I have someone to turn to } \\
\text { for support when needed }\end{array}$ & Occasionally & Often & $\begin{array}{c}\text { Most/All } \\
\text { the time }\end{array}$ \\
\hline $\begin{array}{l}\text { I have felt O.K. about myself } \\
\text { I have felt totally lacking in energy } \\
\text { and enthusiasm }\end{array}$ & $\begin{array}{l}\text { Most/All } \\
\text { the time }\end{array}$ & $\begin{array}{l}\text { Most/All } \\
\text { the time }\end{array}$ & $\begin{array}{l}\text { Most/All } \\
\text { the time } \\
\text { the time }\end{array}$ \\
\hline $\begin{array}{l}\text { I have been physically violent to } \\
\text { others }\end{array}$ & Not at all & Not at all & Not at all \\
\hline $\begin{array}{l}\text { I have felt able to cope when things } \\
\text { go wrong }\end{array}$ & $\begin{array}{l}\text { Most/All } \\
\text { the time }\end{array}$ & Sometimes & Often \\
\hline $\begin{array}{l}\text { I have been troubled by aches, pains } \\
\text { or other physical problems }\end{array}$ & $\begin{array}{l}\text { Most/All } \\
\text { the time }\end{array}$ & $\begin{array}{l}\text { Most/All } \\
\text { the time }\end{array}$ & $\begin{array}{l}\text { Most/All } \\
\text { the time }\end{array}$ \\
\hline I have thought of hurting myself & Not at all & Sometimes & Not at all \\
\hline
\end{tabular}




\begin{tabular}{|c|c|c|c|}
\hline $\begin{array}{l}\text { Talking to people has felt too much } \\
\text { for me }\end{array}$ & Not at all & Sometimes & $\begin{array}{l}\text { Most/All } \\
\text { the time }\end{array}$ \\
\hline $\begin{array}{l}\text { Tension and anxiety have prevented } \\
\text { me doing important things }\end{array}$ & Not at all & Occasionally & Not at all \\
\hline $\begin{array}{l}\text { I have been happy with the things I } \\
\text { have done }\end{array}$ & Sometimes & Sometimes & Sometimes \\
\hline $\begin{array}{l}\text { I have been disturbed by unwanted } \\
\text { thoughts and feelings }\end{array}$ & Occasionally & $\begin{array}{l}\text { Most/All } \\
\text { the time }\end{array}$ & $\begin{array}{l}\text { Most/All } \\
\text { the time }\end{array}$ \\
\hline I have felt like crying & Not at all & Sometimes & $\begin{array}{l}\text { Most/All } \\
\text { the time }\end{array}$ \\
\hline I have felt panic or terror & Not at all & Not at all & Not at all \\
\hline I made plans to end my life & Not at all & Not at all & Not at all \\
\hline $\begin{array}{l}\text { I have felt overwhelmed by my } \\
\text { problems }\end{array}$ & Not at all & Occasionally & $\begin{array}{l}\text { Most/All } \\
\text { the time }\end{array}$ \\
\hline $\begin{array}{l}\text { I have had difficulty getting to sleep } \\
\text { or staying asleep }\end{array}$ & Sometimes & Often & $\begin{array}{l}\text { Most/All } \\
\text { the time }\end{array}$ \\
\hline $\begin{array}{l}\text { I have felt warmth or affection for } \\
\text { someone }\end{array}$ & Not at all & Not at all & $\begin{array}{l}\text { Most/All } \\
\text { the time }\end{array}$ \\
\hline $\begin{array}{l}\text { My problems have been impossible } \\
\text { to put to one side }\end{array}$ & Not at all & $\begin{array}{l}\text { Most/All } \\
\text { the time }\end{array}$ & $\begin{array}{l}\text { Most/All } \\
\text { the time }\end{array}$ \\
\hline $\begin{array}{l}\text { I have been able to do most things I } \\
\text { needed to }\end{array}$ & Not at all & Occasionally & Occasionally \\
\hline $\begin{array}{l}\text { I have threatened or intimidated } \\
\text { another person }\end{array}$ & Not at all & Not at all & Not at all \\
\hline I have felt despairing or hopeless & Not at all & $\begin{array}{l}\text { Most/All } \\
\text { the time }\end{array}$ & Sometimes \\
\hline $\begin{array}{l}\text { I have thought it would be better if I } \\
\text { were dead }\end{array}$ & Sometimes & Sometimes & $\begin{array}{l}\text { Most/All } \\
\text { the time }\end{array}$ \\
\hline I have felt criticised by other people & Not at all & Occasionally & $\begin{array}{l}\text { Most/All } \\
\text { the time }\end{array}$ \\
\hline I have thought I have no friends & $\begin{array}{l}\text { Most/All } \\
\text { the time }\end{array}$ & $\begin{array}{l}\text { Most/All } \\
\text { the time }\end{array}$ & Not at all \\
\hline
\end{tabular}




\begin{tabular}{|c|c|c|c|}
\hline I have felt unhappy & Sometimes & Often & $\begin{array}{l}\text { Most/All } \\
\text { the time }\end{array}$ \\
\hline $\begin{array}{l}\text { Unwanted images or memories have } \\
\text { been distressing me }\end{array}$ & Not at all & Occasionally & Not at all \\
\hline $\begin{array}{l}\text { I have been irritable when with other } \\
\text { people }\end{array}$ & Not at all & Not at all & Not at all \\
\hline $\begin{array}{l}\text { I have thought I am to blame for my } \\
\text { problems and difficulties }\end{array}$ & Not at all & $\begin{array}{l}\text { Most/All } \\
\text { the time }\end{array}$ & Not at all \\
\hline I have felt optimistic about my future & Sometimes & Sometimes & Sometimes \\
\hline $\begin{array}{l}\text { I have achieved the things I wanted } \\
\text { to }\end{array}$ & Not at all & Sometimes & Not at all \\
\hline $\begin{array}{l}\text { I have felt humiliated or shamed by } \\
\text { other people }\end{array}$ & Not at all & $\begin{array}{l}\text { Most/All } \\
\text { the time }\end{array}$ & $\begin{array}{l}\text { Most/All } \\
\text { the time }\end{array}$ \\
\hline $\begin{array}{l}\text { I have hurt myself physically or taken } \\
\text { dangerous risks with my health }\end{array}$ & Not at all & Not at all & Not at all \\
\hline Total Score & $\begin{array}{c}11.8 \\
\text { (Mild) }\end{array}$ & $\begin{array}{c}21.5 \\
\text { (Moderate } \\
\text { Severe) }\end{array}$ & $\begin{array}{c}18.8 \\
\text { (Moderate) }\end{array}$ \\
\hline
\end{tabular}

Table 13 - Jess' CORE-OM Data

The following appear to relate to Jess' responses to items relating to her thoughts, emotions and treatment from others. Jess rated that she experienced an increase in unwanted thoughts and feelings and thought she would be better off dead. This appears to suggest that Jess had difficulties with some of her thoughts and feelings, and that on occasions might not have considered her life worth living. Jess rated that there was an increase in her unhappiness and that she would regularly cry, this may suggest that something occurred within these months which was a source of sadness. Responses suggest that Jess lacked energy and enthusiasm, which appeared to be a residual area of difficulty for her and suggests that interventions based upon motivation might be valuable. Jess also rated that she felt humiliated or shamed by others and criticised by others. 
Lastly Jess rated her ability to cope with her problems as getting worse over this threemonth period. This was represented by decreased ratings of the following items: her perceived ability to cope when things went wrong, her feeling overwhelmed by her problems and her inability to put her problems to one side. This may suggest that developing coping skills may be beneficial, so Jess feels able and confident to manage her problems in the future. However, it may be that solving or improving Jess' problems were not within her control, and therefore consideration of other factors, such as social issues, may be necessary to understand what problems Jess was facing.

\subsubsection{VOICE}

Jess completed the VOICE on one occasion; therefore, I was not able to look at changes over time, nor establish a bassline score as it was not completed upon admission. Jess rated that she agreed that Woodlands was fulfilling all items of the VOICE measure, these ratings varied from strongly agree to slightly agree (see Table 14). This suggests that in April 2019 there were no specific areas of Woodlands' service delivery that Jess rated as not being present or available for her to access if needed. It should be noted that this section only relates to one data point, and therefore this could have changed further into her admission.

\begin{tabular}{|l|c|}
\hline \multicolumn{1}{|c|}{ VOICE } & April $\mathbf{2 0 1 9}$ \\
\hline Made to feel welcome on arrival & Agree \\
\hline I have a say in my care and treatment & Slightly Agree \\
\hline Ward rounds are useful for me & Slightly Agree \\
\hline I feel medication helps me & Slightly Agree \\
and side effects & Slightly Agree \\
\hline $\begin{array}{l}\text { Staff give me medication instead of talking to } \\
\text { me }\end{array}$ & Slightly Agree \\
\hline Staff take an interest in me & Slightly Agree \\
\hline Staff are available to talk when I need them & Agree \\
\hline I trust the staff to do a good job & Agree \\
\hline $\begin{array}{l}\text { I feel that staff understand how my illness } \\
\text { affects me }\end{array}$ & Slightly Agree \\
\hline
\end{tabular}




\begin{tabular}{|l|c|}
\hline I feel staff treat me with respect & Agree \\
\hline $\begin{array}{l}\text { I think the activities on the ward meet my } \\
\text { needs }\end{array}$ & Slightly Agree \\
\hline I find the one-to-one time with staff useful \\
\hline $\begin{array}{l}\text { I find it easy to keep in contact with family } \\
\text { while I'm on the ward }\end{array}$ & Slightly Agree \\
\hline $\begin{array}{l}\text { I feel safe on the ward } \\
\text { I feel staff respond well when the panic alarm } \\
\text { goes off }\end{array}$ & Agree \\
\hline $\begin{array}{l}\text { I feel staff respond well when I tell them I'm in } \\
\text { crisis }\end{array}$ & Agree \\
\hline $\begin{array}{l}\text { I feel able to practice my religion whilst I'm in } \\
\text { hospital }\end{array}$ & Agree \\
\hline I think staff respect my ethnic background & Strongly Agree \\
\hline \begin{tabular}{l} 
Total Score \\
\hline
\end{tabular}
\end{tabular}

Table 14 - Jess' VOICE Data

\subsection{Jade}

Jade was admitted in the Summer of 2018 and had been at Woodlands for 1 year and 6 months at the end of data collection. The data available for Jade consisted of two ReQoL10 measures and two VOICE measures.

\subsubsection{ReQoL-10}

Jade's ReQoL-10 data was not collected upon admission, and the first available data was not until a few months into admission, and then 7 months thereafter. From the data available there appeared to be a deterioration in Jade's rated QoL between the two timepoints (see Table 15). Jade's total score decreased by a total of 12, denoting a reliable change that cannot be attributed to chance or error. I am unable to determine whether this was a consistent deterioration, or whether April 2019 was a one-off; however, I will compare the two datasets to identify differences between how Jade rated each item. 


\begin{tabular}{|l|c|c|}
\hline \multicolumn{1}{|c|}{ ReQoL-10 } & September $\mathbf{2 0 1 8}$ & April $\mathbf{2 0 1 9}$ \\
\hline $\begin{array}{l}\text { I found it difficult to get started with } \\
\text { everyday tasks }\end{array}$ & Most/All the time & Most/All the time \\
\hline I felt able to trust others & Most/All the time & Occasionally \\
\hline I felt unable to cope & Occasionally & None of the time \\
\hline I could do the things I wanted to do & Often & None of the time \\
\hline I felt happy & Occast/All the time & Most/All the time \\
\hline I thought my life was not worth living & Most/All the time & None of the time \\
\hline I enjoyed what I did & Most/All the time & Most/All the time \\
\hline I felt hopeful about my future & Occasionally & Most/All the time \\
\hline I felt lonely & Often & Most/All the time \\
\hline I felt confident in myself & Slight Problems & No problems \\
\hline Physical health problems & $\mathbf{3 4}$ & $\mathbf{2 2}$ \\
\hline Total Score & &
\end{tabular}

Table 15 - Jade's ReQoL-10 Data

Jade rated that her confidence, happiness, hopefulness for the future, and physical health problems improved between September 2018 and April 2019. An increase in her confidence suggests her ability to trust in or have faith in her own ability developed over time. An increase in Jade's rating of her happiness suggests that she was more content in April 2019 compared to September 2018. Jade's increased rating of hopefulness could be indicative of where she is in her recovery and that she was able to start look forward to the future. Jade rated her physical health problems as improving. However, based on this data I am unable to determine what may have contributed to these improved ratings, nor whether these improvements occurred and lasted over a long period of time.

There were also items that Jade rated more negatively, which appear to have contributed to the deterioration in her QoL total score. One item related to a decrease in how Jade rated her trust toward others. This was further supported within her VOICE data which saw a decrease in her trust in staff to do their job (see Table 16). This may suggest that factors or experiences that occurred between September 2018 and April 2019 contributed 
to a reduction in her trust of others. Jade rated her trust in staff to do their job also decreased, which may suggest that Jade had been let down by staff at Woodlands, or previously by staff in another mental health service which had impacted her ability to trust them to fulfil their job role.

Jade's responses to the items "doing the things that she wanted to do" and "enjoying what activities she engaged in" decreased, suggesting that Jade perceived there to be some form of restriction upon what she wanted to do. This could suggest that attempts to support service-users in participating in activities they chose, through promoting choice, may not have been Jade's experience at these timepoint. Jade's VOICE data also suggested there had been a decrease in her rating of activities meeting her needs (see Table 14). This may suggest that there was a discrepancy between what staff perceived to be Jade's needs and her understanding of her needs, meaning the activities offered did not align with what Jade felt was necessary for her recovery. However, this question related specifically to whether the activities that occurred on the ward met her needs, and PAs and staff encouraged service-users to access mainstream, community-based opportunities, which are not applicable in the wording of this item.

There was an increase in Jade's rating of loneliness between September 2018 and April 2019. This suggests that there was a discrepancy between the relationships she had and the relationships she wanted. Jade rated that thinking her life was not worth living had increased between the two timepoints. This is of interest as Jade rated that she was hopeful for the future on both occasions. This may suggest that at the time of completing the measure, Jade did not see value or purpose in that present moment, which could be due to being detained in a locked service but was able to see it in her future.

Lastly, Jade rated that there were no improvements in her ability to get started with everyday tasks, suggesting that this was an area of residual difficulty for her. This may highlight a need for motivational support to help Jade in this area.

\subsubsection{VOICE}

Jade's VOICE data was completed in the same months as her ReQoL-10 and CORE-OM. Her ratings in the VOICE measure indicated that her perception of Woodlands was more 
negative in April 2019 when comparing to September 2018 reflected by an increase of 11 in her total score (see Table 16). This is a similar trend to what was seen within Jade's ReQoL-10 data which also showed her total score had worsened from the first timepoint to the last (see Table 15).

I am only able to compare these two months so do not know if this was a consistent decline in her ratings, or whether April 2019 was a one-off in terms of deterioration in scoring. I can, however, look at declines, improvements or no changes between the two different timepoints available to me.

\begin{tabular}{|c|c|c|}
\hline VOICE & $\begin{array}{l}\text { September } \\
2018\end{array}$ & April 2019 \\
\hline Made to feel welcome on arrival & Strongly Agree & Strongly Agree \\
\hline I have a say in my care and treatment & Strongly Agree & Slightly Agree \\
\hline Ward rounds are useful for me & Agree & Slightly Agree \\
\hline I feel medication helps me & Agree & Strongly Agree \\
\hline $\begin{array}{l}\text { I have an opportunity to discuss my } \\
\text { medication and side effects }\end{array}$ & Strongly Agree & Strongly Agree \\
\hline $\begin{array}{l}\text { Staff give me medication instead of talking to } \\
\text { me }\end{array}$ & $\begin{array}{l}\text { Strongly } \\
\text { Disagree }\end{array}$ & $\begin{array}{l}\text { Strongly } \\
\text { Disagree }\end{array}$ \\
\hline Staff take an interest in me & Strongly Agree & Strongly Agree \\
\hline Staff are available to talk when I need them & Strongly Agree & Strongly Agree \\
\hline I trust the staff to do a good job & Strongly Agree & Slightly Agree \\
\hline $\begin{array}{l}\text { I feel that staff understand how my illness } \\
\text { affects me }\end{array}$ & Strongly Agree & Slightly Agree \\
\hline I feel staff treat me with respect & Strongly Agree & Strongly Agree \\
\hline $\begin{array}{l}\text { I think the activities on the ward meet my } \\
\text { needs }\end{array}$ & Disagree & $\begin{array}{l}\text { Strongly } \\
\text { Disagree }\end{array}$ \\
\hline I find the one-to-one time with staff useful & Strongly Agree & Strongly Agree \\
\hline $\begin{array}{l}\text { I find it easy to keep in contact with family } \\
\text { while I'm on the ward }\end{array}$ & Strongly Agree & Slightly Agree \\
\hline I feel safe on the ward & Strongly Agree & Strongly Agree \\
\hline $\begin{array}{l}\text { I feel staff respond well when the panic } \\
\text { alarm goes off }\end{array}$ & Strongly Agree & Agree \\
\hline $\begin{array}{l}\text { I feel staff respond well when I tell them I'm } \\
\text { in crisis }\end{array}$ & Strongly Agree & Agree \\
\hline
\end{tabular}




\begin{tabular}{|l|c|c|}
\hline $\begin{array}{l}\text { I feel able to practice my religion whilst I'm in } \\
\text { hospital }\end{array}$ & Strongly Agree & Strongly Agree \\
\hline I think staff respect my ethnic background & Strongly Agree & Strongly Agree \\
\hline Total Score & $\mathbf{3 0}$ & $\mathbf{4 1}$ \\
\hline
\end{tabular}

Table 16 - Jade's VOICE Data

Jade strongly agreed with most items within the VOICE measure, which suggests she perceived Woodlands to be fulfilling these areas of service delivery. However, there were some items which changed over time. The only VOICE item that Jade rated as improving between September 2018 and April 2019 related to her view on the helpfulness of medication. This may suggest that the medication she was taking at Woodlands was benefitting her, or her attitude towards taking medication became more positive.

Jade responses suggest that she did not feel staff understood how her illness affected her, which suggests there may have been challenges in Jade and staff establishing a relationship in which these conversations could occur. Additionally, her lack of trust in staff to do their jobs and her trust in others (see Table 16) could have prevented a therapeutic relationship between Jade and staff from forming or being maintained.

Jade rated that staff's response to panic alarms and when she was in crisis decreased, suggesting that there was a discrepancy between the support Jade received from staff, and what she felt she needed when she was in crisis. Lastly, Jade rated that her ability to maintain contact with her family decreased between September 2018 and April 2019, which may suggest that Woodlands did not have the necessary structures in place for Jade to continue this contact.

\subsection{Erin}

Erin was admitted in the summer of 2019 and at the end of data collection had been at Woodlands for 6 months. The data available for Erin consisted of one CORE-OM. No ReQoL-10 or VOICE data was available for Erin. 


\subsubsection{CORE-OM}

The available data for Erin's CORE-OM was collected upon admission, meaning there was no follow-up data available to monitor changes over time. This data may therefore provide an indication of the type of service-users that were being accepted at Woodlands at this timepoint. Erin's CORE-OM total score categorised her psychological distress as moderate (see Table 17).

\begin{tabular}{|l|c|}
\hline \multicolumn{1}{|c|}{ CORE-OM } & June 2019 \\
\hline I have felt terribly alone and isolated & Sometimes \\
\hline I have felt tense, anxious or nervous & Occasionally \\
\hline I have felt I have someone to turn to for support when & Most/All the time \\
\hline I have felt O.K. about myself & Often \\
\hline I have felt totally lacking in energy and enthusiasm & Sometimes \\
\hline I have been physically violent to others & Often \\
\hline I have felt able to cope when things go wrong & Sometimes \\
\hline $\begin{array}{l}\text { I have been troubled by aches, pains or other physical } \\
\text { problems }\end{array}$ & Often \\
\hline I have thought of hurting myself & Not at all \\
\hline Talking to people has felt too much for me & Occasionally \\
\hline $\begin{array}{l}\text { Tension and anxiety have prevented me doing important } \\
\text { things }\end{array}$ & Occasionally \\
\hline I have been happy with the things I have done & Occasionally \\
\hline I have been disturbed by unwanted thoughts and feelings & Occasionally \\
\hline I have felt like crying & Sometimes \\
\hline I have felt panic or terror & Not at all \\
\hline I made plans to end my life & Often \\
\hline
\end{tabular}




\begin{tabular}{|l|c|}
\hline I have felt warmth or affection for someone & Sometimes \\
\hline My problems have been impossible to put to one side & Sometimes \\
\hline I have been able to do most things I needed to & Sometimes \\
\hline I have threatened or intimidated another person & Sometimes \\
\hline I have felt despairing or hopeless & Sometimes \\
\hline I have thought it would be better if I were dead & Occasionally \\
\hline I have felt criticised by other people & Most/All the time \\
\hline I have thought I have no friends & Sometimes \\
\hline I have felt unhappy & Sometimes \\
\hline Unwanted images or memories have been distressing me & Not at all \\
\hline I have been irritable when with other people & Sometimes \\
\hline I have thought I am to blame for my problems and difficulties & Sometimes \\
\hline I have felt optimistic about my future & Sometimes \\
\hline I have achieved the things I wanted to & Often \\
\hline I have felt humiliated or shamed by other people & (Modimes \\
\hline I have hurt myself physically or taken dangerous risks with & Somer \\
\hline
\end{tabular}

Table 17 - Erin's CORE-OM Data

Although I cannot look at changes in how Erin rated the CORE-OM I can look at certain areas that she rated favourably or unfavourably in relation to her psychological distress. The first item related to Erin having no friends. The lack of perceived support provides evidence that Erin may require provisions to increase her social support network. Despite Erin rating that she had no friends, she did feel as though she had someone to turn to when needed. This suggests that Erin did have some form of support that she felt able to rely upon. This suggests that although she may not have support in the form of a friendship, she does have other support, which are not within the capacity of a friendship, 
such as family or staff support. As the CORE-OM was completed upon Erin's admission her rating of having no friends could also be due to the significant change that occurred in this month which saw her moved to a locked service, which could have influenced her friendships, and also meant she had not had sufficient time to establish new friendships at Woodlands.

Erin often felt overwhelmed by her problems, suggesting she may need support to develop coping skills to manage any difficulties she may experience, or her problems were out of her control. Also, the timing of the measure coinciding with her admission to Woodlands, could have been contributing to her feeling overwhelmed, or unable to cope due to the significant change that occurred in her life through being admitted to Woodlands.

Lastly, Erin rated that she was often violent to others. This highlights a potential challenge staff may need to negotiate when promoting recovery, but also balancing the need to maintain the safety of service-users, staff and the public. This highlights that there may be the potential for ethical dilemmas in managing violence, but also promoting recovery at Woodlands. Rating violence towards others as often upon her admission may reflect the types of behaviours of the subset of service-users who are likely to be referred to Woodlands.

\subsection{Gemma}

Gemma was admitted in the Autumn of 2019 and had been at Woodlands for 3 months at the end of data collection. The data available for Gemma consisted of one ReQoL-10 measure and one VOICE measure, which were both completed in the same month of her admission. There was no follow-up data available, nor was there any CORE-OM data available for Gemma.

\subsubsection{ReQoL-10}

The ReQoL-10 measure was completed upon Gemma's admission which provided a baseline; however, there was no follow-up data available (see Table 18). Due to this I was unable to consider any changes over time. I can, however, look at how Gemma rated 
certain items and consider what this may mean in relation to her potential needs and her QoL upon admission.

\begin{tabular}{|l|c|}
\hline \multicolumn{1}{|c|}{ ReQoL-10 } & September $\mathbf{2 0 1 9}$ \\
\hline I found it difficult to get started with everyday tasks & Sometimes \\
\hline I felt able to trust others & Most/ All the time \\
\hline I felt unable to cope & Occasionally \\
\hline I could do the things I wanted to do & Sometimes \\
\hline I felt happy & Occasionally \\
\hline I thought my life was not worth living & None of the time \\
\hline I enjoyed what I did & Sometimes \\
\hline I felt hopeful about my future & Sometimes \\
\hline I felt lonely & Sometimes \\
\hline I felt confident in myself & Sometimes \\
\hline Physical health problems & Moderate Problems \\
\hline Total Score & $\mathbf{2 6}$ \\
\hline
\end{tabular}

Table 18 - Gemma's ReQoL-10 Data

One item that Gemma rated favourably was thinking her life was worth living, which suggests that she saw meaning and value in her life, which may reflect where Gemma was in her recovery. Gemma rated that she felt able to trust others, which could be beneficial in her being able to develop therapeutic relationships with staff.

Gemma rated that she was unhappy upon her admission to Woodlands, which could be due to the significant change that occurred in her life, through being admitted to an inpatient service. Gemma only occasionally felt able to cope, which may suggest this an area in which she requires support to develop strategies and skills, so she feels able to do this in the future. Lastly, Gemma had moderate problems with her physical health. I am unable to consider why Gemma rated the items in this way, but it does allow me to consider how she rated her QoL upon admission to Woodlands.

\subsubsection{VOICE}

Gemma's VOICE measure was completed close to admission (see Table 19). It was intended that the VOICE measure would be completed one month into admission, and 
every 6 months thereafter, to allow enough time for service-users to experience Woodlands before completing a measure relating to their perception of the service.

Gemma only disagreed with the statement that it was easy to keep in contact with her family. Whilst I do not know the reasons for Gemma rating this item in this way, it is important to note that Gemma was only just admitted to Woodlands and therefore, she may not have been familiar with the procedures surrounding maintaining contact with her family. Additionally, her admittance to Woodlands made this contact more difficult, such as being further away by distance. As Gemma rated that Woodlands fulfilled all the other items within the VOICE measure, this may suggest that Gemma's initial experience at Woodlands was favourable. Due to no follow-up data being available I am unable to determine whether her view of the service changed during her admission.

\begin{tabular}{|l|c|}
\hline \multicolumn{1}{|c|}{ VOICE } & $\begin{array}{c}\text { September } \\
\mathbf{2 0 1 9}\end{array}$ \\
\hline Made to feel welcome on arrival & Agree \\
\hline I have a say in my care and treatment & Agree \\
\hline Ward rounds are useful for me & Slightly Agree \\
\hline I feel medication helps me & Agree \\
\hline $\begin{array}{l}\text { I have an opportunity to discuss my medication and side } \\
\text { effects }\end{array}$ & Disagree \\
\hline Staff give me medication instead of talking to me & Agree \\
\hline Staff take an interest in me & Agree \\
\hline Staff are available to talk when I need them & Agree \\
\hline I trust the staff to do a good job & Slightly Agree \\
\hline I feel that staff understand how my illness affects me & Agree \\
\hline I feel staff treat me with respect & Agree \\
\hline I think the activities on the ward meet my needs & Agree \\
\hline I find the one-to-one time with staff useful & Disagree \\
\hline $\begin{array}{l}\text { I find it easy to keep in contact with family while I'm on the } \\
\text { ward }\end{array}$ & Agree \\
\hline I feel safe on the ward & Agree \\
\hline I feel staff respond well when the panic alarm goes off & Agree \\
\hline I feel staff respond well when I tell them I'm in crisis & \\
\hline
\end{tabular}




\begin{tabular}{|l|c|}
\hline I feel able to practice my religion whilst I'm in hospital & Agree \\
\hline I think staff respect my ethnic background & Agree \\
\hline Total Score & $\mathbf{4 6}$ \\
\hline
\end{tabular}

Table 19 - Gemma's VOICE Data

\subsection{Summary}

I have presented each service-user as a separate case study to identify and discuss changes over time. I will now consider the complete dataset. Caution was used throughout when considering potential trends, meaning no inferences were drawn. The practical limitations of the data available to me meant I was cautious of over interpreting the data (see section 8.5.3 for further discussion of limitations). Throughout the data there was discrepancies between what was done at Woodlands, and the agreed protocol, this was represented by the random fashion in which data appeared to be collected. The assurances I was given, by the gatekeeper and staff, have not proved consistent with practice at Woodlands.

It is well established that small scale quantitative studies may be less reliable because of the low quality of data which affects the ability to generalise findings to wider populations, commonly known as inferences (Denscombe, 2010). Also, there was limited follow-up data to be able to demonstrate an effect which could increase the likelihood of me drawing a biased inference (Altman, 2001). Due to these limitations I chose to use descriptive statistics to point out interesting patterns (Newing, 2019).

Whilst it was not communicated to me why the decision to cease data collection was made, it shows how imperative it is that routinely collected data is embedded within the design of the service and is adequately communicated to staff. If the completion of routinely collected data is left to staff interpretation or simply staff remembering to do them, then it may not be operationalised as intended and the opportunity of monitoring and documenting progress lost. As Woodlands did not operationalise regular, routine standardised measures of recovery, this data was not available as a means of documenting and collating service-user progress and evidencing the effectiveness of 
Woodlands. The lack of evidence was a key factor that commissioners stated Woodlands was missing compared to other providers and which contributed to the low referrals (see section 4.5.1.1.4). Therefore, it appears that the collection of data was not embedded into the day-to-day operation of Woodlands. Furthermore, the lack of data may slow down service-user progress and Woodland's ability to show there has been a change or improvement overtime, which is needed for a service-user's section to be removed. This highlights the importance of systems being put in place to ensure routinely collected data becomes part of the operations at Woodlands.

The above limitations mean that I am only able to make summations about the people that completed the measures at Woodlands. In these summations it appears that serviceusers rated key recovery processes as low, or their ratings reduced in over time.

I present a preliminary and necessarily limited set of postulations based on my analysis of standardised measures used at Woodlands. I recognise in doing so that trends are difficult to discern, and robust or reliable conclusions cannot be made from the data available. I acknowledge that the data and the trends are limited, as are the potential conclusions drawn; however, I shine a light on some of the interesting patterns across the quantitative data and position these alongside current recovery literature.

In section 8.3.1, I address the research objectives of this project and I rely heavily upon the qualitative findings. Due to this, I felt it was necessary to have a separate section where I focus upon the trends of the quantitative data so that these results did not get lost when integrating the two datasets to address the research objectives.

\subsubsection{Positively Rated Items}

There were two areas which were rated positively across the quantitative data. The first related to service-user's ability to remain in contact with their family. Family have been identified as playing a central role in promoting confidence and hope during an individual's recovery (Deegan, 1988; Kelly \& Gamble, 2005) and their involvement has been widely advocated within mental health policies (Pharoah et al., 2010). It appears that most of the service-users who completed the measures felt that they were able to 
maintain contact with family; suggesting there were structures in place which aided their ability to do this.

Secondly, service-users valued one-to-one contact with staff; which appears to align with the emphasis PAs placed upon ensuring certain staff qualities were present to ensure therapeutic relationships formed. However, some service-users did not feel that the staff were always accessible. The availability of nurses has been recognised elsewhere as a valuable component of service delivery for service-users (Coffey et al., 2019a) and they regularly report that staff are too busy to provide support (Kornhaber et al., 2016). In practice, the availability of staff is often diluted by non-patient-based responsibilities (Hopkins et al., 2009), restricting nurses to only working therapeutically with service-users for just four to twenty percent of their time (Sharac et al., 2010). This is of interest given the fact PAs designed the staffing model as a means of improving staff accessibility (see section 5.4). In practice, based upon service-users' quantitative ratings it appears that opportunities for access did not match up with their expectations or needs. This highlights that the support of staff, both in terms of one-to-one contact and the accessibility of this support, appeared to be a much-needed resource for the service-users. There appears to be divergence between service-users' quantitative ratings of staff accessibility and the qualitative data which suggested there were too many staff on shift and that the ratio of staff to service-users was higher than anticipated due to the low referral numbers. This could suggest that although there was a physical presence of too many staff at Woodlands, service-users did not feel they were able to access and use this resource as they desired. It may be beneficial if Woodlands could reconsider how best to use their staffing numbers to their advantage, particularly in the context of low service-user numbers, when the resource of high staff levels should arguably be at its most accessible.

\subsubsection{Hope}

The data available highlighted hopelessness was prevalent amongst the individuals who completed the measures. There was a trend that suggested for service-users who had completed the same measure at different time points, that their ratings showed them becoming more hopeless over time. 
A review of service-user perspectives of recovery identified four key processes, one of which was hope (Andresen et al., 2003). Hope is often cited in recovery narratives as a crucial catalyst in instigating the recovery journey (Anthony, 1993) and is a maintaining factor for recovery (Schrank et al., 2011). Deegan (1988) stated that:

'It is important to understand that for most of us recovery is not a sudden conversion experience. Hope does not come to us as a sudden bolt of lightning that jolts us into a whole new way of being. Hope is the turning point that must quickly be followed by the willingness to act.'

Her account highlights that hope is a cornerstone of recovery, and where the situation begins to change for the individual. For service-users, 'recovery is about hope' (Storey et al., 2008, p. 3).

The routinely collected data from Woodlands indicated that service-users had low scores for hopefulness. Whilst I am unable to explain the reasons items relating to hope were rated in this way, it may highlight where service-users were in their recovery journey. It is also known that hope fluctuates (Onken et al., 2007); due to the irregularity of the data available, I was unable to see whether service-user ratings reflected fluctuations, or whether their sense of hopelessness was persistent. This highlights that the scores do not present a full picture of what was going on for service-users at Woodlands in relation to hope, and only reflect a snapshot in time.

Slade et al (2012, p. 101) suggested that 'hope, without opportunity, dies'. Thus, meaning that a paradox of hope may arise in the face of limited chances to achieve a life they value (Mattingly, 2010). Not only does hope need to be 'followed by the willingness to act' (Deegan, 1988), but service-users also need opportunities to act upon their hope. It has been suggested that there is a back and forth spiralling movement towards and away from hope, and this movement can be shaped by the social environment (Mattingly, 2010). The qualitative data suggested that in some instances access to certain opportunities, such as educational courses at college, were restricted due to their experiences of stigma (see section 6.3.2). Staff participants claimed that they had to adapt their approach to community-based opportunities in response to this stigma, meaning service-users were limited to mental health specific options. However, this is only one hypothesis for the 
ratings of hopelessness that is drawn from the qualitative data, but this would require further research and analysis.

Service-users rated other items that are correlated with low hope, indicating that the scores reflect a wider sense of hopelessness. The constellation of lack of optimism for the future, thinking their life was not worth living, physically harming themselves and having a plan to end their life are highly correlated with completed or attempted suicides (Beradelli et al., 2019). This may highlight where the individuals at Woodlands were in their recovery, reflected by a lack of hope and optimism.

To summarise, responses from service-users at Woodlands suggested that they felt hopeless, and analysis of the data available suggests that this hopelessness became worse over time. I have shown that within the literature, hope was positioned as a key recovery process (Deegan, 1988). I acknowledge that I did not have access to baseline scores, or historical scores of service-users to provide an understanding of whether hopelessness and a lack of optimism were residual difficulties for these individuals. The ratings relating to hope may reflect the subset of individuals who are needing of services like Woodlands. Woodlands faith in their recovery model as being the answer to these challenges may be optimistic at best, until they are able to demonstrate evidence that it can achieve what they believe it can, such as supporting service-users to have hope and optimism for the future. Whilst I cannot make assumptions about why these items were rated in this way, it does highlight that hope, or a lack of it, formed part of the context in which Woodlands was operating and therefore this could have influenced how service-users engaged with resources at the service.

\subsubsection{Trust}

The data available suggested that, for service-users who completed the measures, their ratings of trust did not improve over time, and on some occasions worsened. It should be noted that this trend related to service-users' trust in others and trust in staff to do their job. I will, therefore, consider trust within both the context of staff relationships and other forms of support. 
Trust has been identified as foundational to the development of a therapeutic relationship between staff and service-users within mental health (Cahill et al., 2013). If trust is not present when attempting to develop a therapeutic relationship it could prevent an alliance from being established (Gilburt et al., 2008). This could be problematic as it is widely accepted, both within literature and practice, that the therapeutic relationship plays a key role in mental health recovery (Andresen et al., 2003). This appears to conflict with PAs description of purposely selecting staff with the qualities, skills and experience to therapeutically engage service-users in their recovery (see section 5.2). When considering trust in the form of other support, service-users rated that they felt they did not have friends, and therefore their opportunities to trust others outside of Woodlands may have been limited. This constellation of items and how service-users rated them suggests that these individuals may have a small support network, or no social support which could be contributing to a lack of opportunity to trust in others, or their trust has been broken within previous relationships.

Provisions that intend to improve trust have been identified in a review by Laugharne et al (2012) such as valued contact and listening time, emotional support, and involving service-users in decisions about their care. It has also been suggested that continuity of care and reliability in delivery can build service-user trust in staff and providers (Laugharne et al., 2012). Participants reported their perception that staff turnover had been high, and it was proposed the current rota system adopted at Woodlands made for irregular staffing. This may have created a context in which the development of trust between staff and service-users could not occur or was hindered, resulting in ratings showing low levels of trust reported by users.

\subsubsection{Meaningful Activities}

Whilst I acknowledge that there is little agreement of what makes an activity meaningful, meaningful occupation has often featured in accounts from individuals with a lived experience of mental illness (Deegan, 1988). The inclusion of activities within care has been linked with increased levels of recovery and wellbeing (Stickley et al., 2018); whereas a lack of activities is linked with deleterious effects, such as service-user boredom, 
frustration and dissatisfaction (Foye et al., 2020). This highlights the importance of service-users having access to meaningful activities.

The data available suggested that service-users who completed the measures did not rate the activities available at Woodlands as meeting their needs, nor were these activities a source of enjoyment. It should be noted that the wording of these items related to what was provided at Woodlands and did not include activities that service-users accessed independently of Woodlands. Alternatively, a focus of Woodlands was to encourage individuals to access community-based opportunities, (see section 6.2) meaning in-house activities were not prioritised and may not have met their needs. This highlights that there may be a need to balance the advantages of mainstream, community-based opportunities, with ensuring activities are available internally for when service-users are not on leave. Alternatively, service-users' needs may be being met by community-based opportunities, but the items on the quantitative measures do not consider these community-based opportunities. As Woodlands promotes community-based and external opportunities, rather than in-house activities, it may be beneficial for the service to find an appropriate and reliable way to collect data that enables service-users to rate these opportunities, as well as providing the service with meaningful data.

\subsubsection{Loneliness}

The data available suggested that service-users who completed the measures, reported feeling lonely, and for those who completed the measures on more than one occasion, this loneliness increased over time.

Foucault (2008) highlighted that imposed isolation has always been justified within the field of psychiatry. This justification often related to the service-users or others safety, to subject service-users to medical care, to isolate them from outside influences and to impose better intellectual and moral habits upon individuals. These reasons are still apparent within mental health settings today, however different language is now used concerning the prevention of suicide, to provide safety and security, and the regulation of service-users and visitors in and out of services. This suggests that mental health inpatient settings often isolate individuals from the wider community, therefore their opportunity to develop or maintain their desired social relationships are impeded. The qualitative data 
suggested that Woodlands was attempting to overcome this isolation by encouraging community-based opportunities, however this was restricted by staff to prevent individuals from experiencing rejection and stigma (see 6.3.2). According to Peplau and Perlman's (1982) conceptualisation of loneliness, people feel lonely if there is a discrepancy between the relationships they have and the ones that they want. Simply being at Woodlands, a locked service, may contribute and restrict the social connections service-users have access to. Individuals may have the limited skills and resources, such as liberty and autonomy, to do something about their situation, which appears to be reflected in ratings of loneliness.

Loneliness has been documented as a problem amongst mental health service-users (Palumbo et al., 2015). It is well-established that social relationships are integral to an individual's wellbeing; and social integration has been found to have protective effects on morbidity and mortality outcomes (Holt-Lunstad et al., 2015). Loneliness has been associated with physical and mental ill-health, including increased depression, anxiety, social withdrawal, suicidal behaviours, and higher use of hospitalisation (Lim et al., 2016; Holt-Lunstad, 2018). This research highlights the deleterious effects of loneliness and the prevalence of this issue within mental health populations. This trend in the data therefore emphasises the importance of services supporting service-users so they can be socially included and participate within society, as loneliness needs to be improved within mental health populations.

Service-user rated loneliness does not align with PAs vision that the staffing model available at Woodlands would ensure that staff were readily accessible (see section 5.4). There may be differences between the availability of social support, as measured by proximity, and the supply of social support in the form of close, or intimate, relationships. It may also highlight that there are differences between how service-users engage with staff, versus other forms of relationships, such as friendships. The trend of loneliness was further reflected in service-user ratings of certain items, such as increased feelings of being alone and feeling they have no friends. Social support has been identified as an aid to recovery (Onken et al., 2007). It has been reported that the size of a person's social network and their satisfaction with their support correlates with recovery (Corrigan et al., 2004) and decreasing scores for social support have been associated with decreased 
quality of life scores (Coffey et al., 2019b). This further emphasises the importance of supporting service-users to develop their social support networks.

The qualitative data highlighted that Woodlands encountered challenges such as stigma and rejection when attempting to support individuals to establish themselves as part of the community and increase their social network. This highlights that despite Woodlands attempts to address and improve loneliness and inclusion, societal factors, that are out of the service's control, can limit the success of these attempts. Service-user ratings of loneliness may have been influenced by both wider social issues that people with mental ill-health face, and by being deprived of their liberty as a result of being detained in inpatient care. This is a challenge that Woodlands are likely to continually encounter, and therefore creative means of overcoming these difficulties are warranted.

\subsubsection{Explanatory Power}

Due to the limitations of the quantitative data, and the fact that these results cannot be attributed to a source or cause, unlike the other chapters I will not present partial, fallible theories. Instead, I will discuss the outcomes of interest in relation to the congruence model and show how this will build upon how the theory has been used within previous chapters. As this chapter represents Woodlands' outputs in relation to individual-level recovery outcomes for service-users, I will consider how the service's inability to create an organisation and strategy that was congruent with current inputs meant that key recovery processes were not facilitated (see Figure 8).

The findings suggests that there is dissonance between the cultural aims and vision of Woodlands to support and promote recovery, and the individual-level outcomes for service-users (see Figure 8). Previous chapters have shown Woodlands culture, people, work and structure were not congruent with the current market demand, policy direction and who was eventually accepted at the service. Therefore, it is unsurprising that recovery outcomes, as measured within routinely collected data, were not as promising as Woodlands would have anticipated or hoped for.

All the trends presented have an evidence-base which highlights how these areas can either support or hinder an individual's mental health recovery. Woodlands has the aim 
of providing ROC and would hope that some of these areas will have improved during an individual's time at the service. This indicates that there were challenges in supporting some of the recovery processes that have been shown to help individuals in their recovery journey. The quantitative findings could be attributable to the fact that staff at Woodlands (people in the congruence model, see Figure 8) were unsure of how to adapt their approach to meet the needs of the unexpected service-user group eventually accepted at Woodlands (tasks in the congruence model, see Figure 8). Due to this, staff did not have the skills, training or confidence to get to a point where they were focusing upon areas of recovery, such as hope, trust and forming social connections, which was reflected in the results presented. This highlights the importance of the necessary structures to be in place at Woodlands, so that staff are supported to adapt the approach and have the resources to be able to fulfil their tasks at work - which the previous chapters suggest was not the case. Due to these organisational issues, staff were unsure of how to work with these individuals, which meant they were unable to get to the 'recovery work', which could be contributing to the quantitative findings which show no change, or a deterioration, in key areas where you would expect improvements from a service aimed at promoting recovery. These findings highlight that when a service is unable to match its design and strategy with current political, social and economic drivers, which are influential inputs in the successfulness of organisations, then this can have negative consequences of individuals accessing these services.

Furthermore, attempting to support and facilitate processes associated with recovery, such as hope, trust and engagement in meaningful activities was likely hindered by the fact Woodlands is operating in a closed setting. My analysis of these data, therefore, raise the question over the compatibility of the concept of recovery, which is based upon freewill and liberty, within inpatient settings.

In the next chapter I will discuss all the findings from Chapter 4-7 in the context of wider literature and consider limitations of the thesis, and areas of future research. 
8 DISCUSSION 


\subsection{Introduction}

In this chapter, the revised CMOCs for the wider mental health referrals system, the staffing model and choice and responsibility will be explored, and the study findings will be discussed in terms of the existing literature on ROC in inpatient settings. I discuss the findings in relation to implications for the service and wider practice, limitations of the project and future research.

\subsection{Recapitulation of the Study's Purpose}

Woodlands is an inpatient service that aims to support service-users in their recovery, thereby enabling them to be discharged into the community. This study has evaluated the service using a RE approach. To evaluate Woodlands, three key areas of practice were researched - the wider referrals system, the staffing model and how service-users made choices and took responsibility. The aim was to determine and test programme theories, expressed as CMOCs, to explain how and in what circumstances the service and key components of the service's vision worked in supporting service-users in their recovery (see section 1.4 for research question and objectives). These findings are useful in gaining new and transferable knowledge about recovery-oriented inpatient services, which would help to develop the limited evidence base.

The overarching research question of this thesis was:

- What works, for whom and in what circumstances at Woodlands in supporting service-user recovery?

To address this research question, I looked at specific aspects of the service delivery at Woodlands. I considered Woodlands as a new resource in the wider mental health system, the staffing model, and choice and responsibility and then discuss what this means for Woodlands as a service. 


\subsection{Main Findings}

I will structure and present the main findings in relation to the research objectives, and I focus upon the CMOCs that were developed throughout this thesis. I attempt to show integration of the qualitative and quantitative datasets and highlight were there are convergent and/or divergent findings and complimentary findings (Farmer et al., 2006; O'Cathain et al., 2010) (see Appendix V). It is important to note that the design of this thesis was primarily qualitative, with a concurrent supplementary quantitative component, therefore, it was always intended that more emphasis would be placed upon the qualitative findings.

The quantitative measures used did not always align with the direction of the realist interviews and due to this, on occasions, I may be discussing areas of similarity as opposed to the same construct. Also, any divergence seen between the datasets could be because the questionnaires asked service-users to rate how they felt, or their experience over a certain time, whereas the interviews allowed for much broader coverage of their time at Woodlands, and afforded a deeper description of the issue being discussed. I have already noted the limitations of the quantitative data available to me, including the deviations from the agreed protocol (see section 2.6.2.1) and being unable to explain why serviceusers rated items in a certain way. Therefore, I was mindful of what could be inferred from the quantitative data and have therefore been cautious during the integration.

\subsubsection{Addressing the Research Objectives}

I now refer to each research objective and integrate the qualitative and quantitative findings to address these.

- To identify the key resources (mechanisms) used at Woodlands and to identify how these resources are working to support service-users in their recovery? (see Chapter 5 and 6). 
- To identify the contexts that are, or are not, conducive for these resources to work as intended in supporting individuals in their recovery (see Chapter 4, 5 and 6).

- To develop programme theories which lead towards un/successful delivery of recovery-oriented care at Woodlands (see Chapter 4,5 and 6 - These are presented as CMOC models within each specific chapter).

\subsubsection{Staffing Qualities}

This resource related to the recruitment of staff with non-paternalistic attitudes and who possessed people skills to therapeutically engage with service-users. The context which influenced whether these qualities worked as anticipated was whether service-users wanted to and had the skills to establish a relationship with staff, and staff had the skills to establish and maintain these relationships (see Figure 10 on page 153). This determined whether the interactions between service-users and staff were positive or challenging.

If both parties had the individual capabilities to develop and maintain a relationship, then staff were able to work with service-users in a way that moved beyond their mental illness where staff viewed them as individuals; leading to positive staff and service-user interactions. Some service-users claimed that staff were the best aspect of Woodlands, and positive accounts of staff qualities were presented within these interviews. This was supported by the routinely collected data which suggested service-users valued their oneto-one contact with staff. However, the quantitative data also suggested that these interactions did not occur frequently enough. Although staff were accessible to serviceusers for them to rate their interactions positively, it does highlight that frequency of these interactions may require consideration by the service.

However, for some service-users, who had more complex difficulties or had difficult life experiences, such as trauma, relying upon staff qualities was insufficient (see Figure 10). This suggests that where developing relationships may be more complicated, staff may require additional training to be able to support these individuals as relying upon their people skills was not enough. Staff clearly require a further set of psychological awareness 
skills, in addition to having people skills, to establish therapeutic relationships with those with more complex difficulties. It is therefore important that Woodlands ensures all staff are supported and trained to manage the unexpected service-user group eventually accepted at the service to support the development of therapeutic relationships. The service may need to consider adapting their recruitment so that individuals with the relevant interests and skill set are employed to meet the needs of all individuals accepted at Woodlands.

The quantitative data also suggested that service-users did not trust others, and some did not trust staff to do their job, which may have influenced the development of therapeutic relationships. These types of ratings were not expressed within the interview data and therefore highlights divergence between the datasets. One possible reason for this divergence could be because qualitative accounts articulated that the power rested with staff, for example as to whether service-user's choices were facilitated and supported. Therefore service-users may have been concerned about voicing negative perceptions of staff within interviews and may have felt more comfortable doing this within the anonymised quantitative measures. However, it could also be because the quantitative measure directly posed the question relating to trust with staff, whereas the research interviews were more general and open ended.

\subsubsection{Staffing Levels}

The resource of staffing levels at a ratio of 1:2 to 1:2.5 staff to service-users was positioned as a key resource at Woodlands and I incorporated the findings relating to this resource into a CMOC (see Figure 12 on page 159). However, the unexpected outcome of referral difficulties (see section 4.5), created a context which meant that staff had more time available to them than expected, but they were unsure of how to use the unanticipated resource of time. This was an interesting finding considering mental health staff often report that time is a limited resource (Coffey et al., 2019a). It appears that although staff at Woodlands had the resource of time, this was also not beneficial for service delivery as there was no shared vision amongst the staff group of how best to use this time. Some staff seemed to use this time to complete the DLT needed, whereas others did not. For those that did complete they were reported as being able to bring people along with them 
and engage them in the DLT (see Chapter 6). This suggests that some staff were able to assertively engage, whereas others waited to be engaged by service-users, for example when they needed help (see Chapter 6), which some participants attributed to staff confidence and ability to complete DLT. Additionally, an "us-and-them" dynamic developed due to the apparent contrast in numbers. Due to a claimed irregular staffing model, some service-users felt unable to form relationships with staff, leading them to feel unsupported and unable to access help when needed (see Figure 12).

This resource highlights the challenges of a service when they are unable to match their staffing model with the number of referrals they receive and accept at the service. The findings suggested that there was variability in how staff responded to the additional resource of time that was gained from having fewer service-users than anticipated. For example, it was claimed that some staff completed DLT on behalf of service-users, whereas others used the time to encourage and motivate service-users. This evidenced how staff characteristics influenced the way in which they engaged with service-users and how they responded to the troubles in securing referrals.

\subsubsection{Peer Mentors}

PMs, individuals with lived experience of mental illness and had previous contact with mental health services, were identified as a key resource of Woodlands, and due to this I developed a $\mathrm{CMOC}$ to reflect how this resource worked at the service (see Figure 14 on page 169).

My analysis showed that PMs operated within a liminal space, they were neither serviceusers, nor professional staff, and were able to interact with service-users in ways that professional staff could not. For some service-users PMs become a source of hope and guidance. However, the quantitative data suggested that service-users at Woodlands were without hope, and their hopelessness increased over time. This may suggest that the inclusion of PMs on their own may not be sufficient to instil or develop a sense of hope amongst service-users, and other sources of hope are needed. Instilling and maintaining hope may be more complicated than including individuals with lived experience as part of the staffing group, and thereby Woodlands may need to find alternative and supportive means of developing hope for service-users. 
One service-user, who valued medical knowledge from professional staff, said they did not value the role of peer support, leading to them only seeing differences between themselves and the PM. This was an original contribution to literature as it shows that including those with lived experience may not be enough for all service-users to see similarities between themselves and the PMs. This resulted in this individual not accessing peer support and seeing the inclusion of PMs as potentially harmful to their recovery, which supports previous literature (Farber, 2006). Some individuals may have been socialised into expecting and accepting medical knowledge within inpatient care, so that when a service tries to incorporate other forms of knowledge, service-users may dismiss this unknown quantity without allowing themselves the space and time to experience it.

There were various responses to the resource of PMs being embedded within the multidisciplinary team at Woodlands (see Figure 14 on page 169). Due to PMs being perceived as part of the team, some service-users were unaware of the role of PMs and some PMs were unable to differentiate themselves from other staff, meaning they could be operating as paraprofessionals and the value of peer support being lost. This was an original contribution to knowledge as it shows the importance of PMs being separate from the clinical team, rather than necessarily incorporated as this may cause confusion in terms of what their role is. Professional staff did not include PMs within consultations and therefore some PMs did not feel their knowledge formed part of the decision-making at Woodlands. This was despite some participants sharing examples of how they claimed to have used their lived experience to work in ways that professional staff could not, such as challenging service-users. This shows that the context of being in a multi-disciplinary team was conducive to peer support when PMs were able to differentiate themselves from other occupations at Woodlands and use their lived experience knowledge to support service-users.

There is well-established literature on organisational science that shows that when new workers are not enabled to bring their unique expertise and practice to their work then role adoption fails (Dierdorff \& Morgenson, 2007). My findings show that although some PMs were able to use their knowledge to work in different ways to professional staff, others were unable to distinguish themselves from professional staff. This suggests that there may have been a blurring of roles and some PMs were operating as 
paraprofessionals, which would undermine the values underpinning peer support. If this is the case, the service must consider how peer mentors can be supported to establish themselves as a distinct occupation at Woodlands. If peer support can remain distinctly social, rather than clinical, then PMs are perhaps uniquely placed to focus upon serviceuser-focused recovery, in the form of community, collectivism and relationships, rather than individual change. However, from my findings and my use of the EST, it is clear for this to be achieved it would require ongoing organisational support and commitment, which represents a novel contribution to literature.

\subsubsection{Service-user Choice}

In Figure 16 (page 191), I present three different contexts that were influential in how choice operated at Woodlands. These contexts were important as the individual characteristics of service-users and the expectations of staff were all claimed by participants to be influential at Woodlands. There was variation amongst service-users as to whether they were able to make choices for themselves, and this related to whether they had limited experience of making choices previously and whether they had the confidence and skills to be able to make choices. For service-users who had limited experiences of choice, they were overwhelmed by the prospect of making choices and required appropriate support from staff to be able to meet this expectation. This meant some would deflect choices back to staff, which has been reported elsewhere in literature (Waldemar et al., 2018a; Waldemar et al., 2018b), or they felt they needed more staff support to be able to make a choice, which some claimed was not always forthcoming. Some staff accounts alluded to an expectation that service-users would be able to make choices for themselves, which meant the promotion of choice was embedded within a context where it was assumed individuals would have the skillset and ability to make choices for themselves, which was not always the case. There was a discrepancy then between the ability of service-users to make choices for themselves and some staff's expectations, meaning that support was not always available when needed. Staff at Woodlands need to find a balance between autonomy and providing the necessary support and resources so that service-users learn the skills needed to make choices for themselves. 
Choice was also contingent upon service-users being ready and able to make decisions for themselves, and that these were considered the 'right' choices. Staff were risk adverse and saw the potential harms in some activities, rather than appreciating the potential benefits. If they saw the benefits, they did not appear to believe these outweighed the possibility of harm. There was also a moral tone to some staff accounts, with the idea that some things, such as substance use, were simply wrong and should not be encouraged. In engaging with these 'wrong' behaviours and choices, service-users opened the way for blame, culpability and in some instances were considered unsuitable or uncommitted to their recoveries. This approach appears to contrast with the idea advanced by the programme architects that people may have to learn as they go along and might still make mistakes, or occasionally have faulty judgement.

For service-users who felt ready to make choices, and had the skills to do this for themselves, some opted to access community-based opportunities, as this was encouraged at Woodlands. An example of a negative experience of stigma was identified by one staff member as influential in how the resource of choice was operationalised in practice. This led staff to adapt the service's approach by restricting choice to mental health-specific opportunities. Restricting service-users to certain choices could be viewed as contributing to ghettoisation (Durocher et al., 2014; Stewart, 2019), as they were limited to opportunities that were only available to those with a mental illness. However, these findings do highlight that staff had to carefully balance potentially exposing individuals to stigma or rejection, which is a unique contribution to the ROC literature. Staff opted to protect service-users by facilitating opportunities were these experiences was less likely. External barriers, such as societal stigma and rejection, are beyond the control of Woodlands, but this finding does highlight a challenge that the service encountered when trying to support individuals in finding a meaningful place within society. Choice is not infinite for anyone, therefore, reduced or limited choice exists for us all. These findings show that this can be compounded for individuals with mental illhealth, as their choices can be further limited by negative societal perceptions of mental illness. This supports the social model of disability and the need for a social model of mental health recovery. 
The recovery journey may involve personal change and the need for individuals to social (re)engagement in the community (Tew et al., 2012). Woodlands located problems largely with the individual so that service-users could get better and be independent of support and services through being able to make the 'right' choices. Through promoting the ideals of autonomy, some might argue that mental illness is positioned as a personal, rather than social, problem. The social model of disability focuses on wider processes in the social mainstream which are used as markers of social difference and can impact individual's mental health (Beresford, 2002). The findings highlight that whilst Woodlands, aimed to support personal change and social (re)engagement within the context of inpatient care, this acceptance and inclusion was not prevalent in all environments the service-users encountered. The findings showed that social factors, such as stigma, influenced how Woodlands supported service-users in their choices and limited the opportunities available to individuals to support their recovery.

\subsubsection{Service-user Responsibility}

In Figure 18 (on page 200), I presented a CMOC to represent what worked, for whom and in what circumstances in relation to DLT. The first contextual factor related to serviceusers who lacked confidence in their ability to complete DLT due to their limited experience of such responsibility within other services. This meant that some serviceusers feared being judged for their skill level and would therefore avoid engaging in opportunities to learn skills through completing DLT. There was variation amongst serviceusers and their responses and engagement in DLT. One characteristic that participants identified was service-user confidence, as this influenced whether they would participate in the activity. A lack of confidence also extended to staff. Staff members who did not feel able to complete the DLT themselves, were, less likely to encourage service-users to participate, meaning individuals did not always have the support or motivation available to overcome their lack of confidence to participate.

For staff who did have the skills and confidence, they were able to encourage and motivate individuals to complete the DLT or would complete these on service-user's behalf. These contextual factors highlight that the individual capabilities of key actors are relevant to its success, namely self-belief and confidence. There is a need for a progressive 
approach where service-users and staff are supported to develop the confidence and motivation needed to complete DLT, rather than expecting individuals to independently do this upon admission.

The expectation of completing DLT was also embedded into the context of a locked, inpatient facility which is governed by policies and procedures. These policies restricted service-users from freely being able to access the equipment needed to complete DLT without asking for staff permission. These organisational barriers were a source of frustration for service-users and led to a reduction in the number of DLT they completed, meaning staff were often completing these tasks. The service needs to find a means of supporting service-users to freely complete DLT, so that the frustration does not result in them no longer developing the skills PAs identified as necessary for independent community living.

Staff participants also identified that the low service-user occupancy at Woodlands meant that service-users were responsible for a higher number of DLTs than anticipated, as a set number of tasks needed to be completed daily. Designing DLT with the idea of full capacity is more service centred as opposed to person-centred. This indicates that Woodlands had a blanket approach in which a range of tasks were considered important for the smooth running of the service, as opposed to these tasks being specifically designed and identified to support the individual skills of each service-user.

The aspects of choice and responsibility discussed at Woodlands were associated with service-user readiness as a key mechanism of change. It showed that the service was underpinned by a behaviour of change theory, such as the transtheoretical model (TTM) (Prochaska \& DiClemente, 1982). Although the findings allude to an importance of a stages of change view of recovery, there are some concerns that need considering. Designing services which are underpinned by psychological theory of change raises the question of whether services are deviating from the true meaning of recovery. Using theories of behaviour change as interventions has been as one way in which services are promoting recovery through a neoliberal lens (Recovery in the Bin, 2019). The TTM positions the individual as the primary driver of behaviour change processes, and although the active role of individuals in their recovery is now widely advocated, this omits factors outside of an individual's control, such as needing a hope-filled environment and ongoing 
community support. The TTM as an underlying theory of Woodlands is only relevant when ambivalence poses a barrier to recovery, as these approaches do not account for instances where individuals are motivated and active in their recovery, yet still encounter barriers to recovery beyond their control, such as discrimination or poverty, as seen within this thesis. The continued effort by services such as Woodlands to use theories that place the solution to mental ill-health firmly with the individual service-user fails to address other real issues and barriers, such as marginalisation, power imbalances and stigma. This thesis highlights that recovery, and key processes associated with recovery were hindered by social problems, which warrants a social solution. This thesis highlights the limitations of services which are grounded in behaviour change theories and raises the question of whether this is an appropriate approach for services which claim to be promoting recovery should be adopted.

\section{- To identify why Woodlands faced challenges when trying to establish itself as a} new service within the mental health referrals system? (See Chapter 4).

My analysis of the data from research interviews with staff, service-users and commissioners centred on identifying what worked, for whom and in what circumstances relating to Woodlands being a new resource and embedded into the pre-existing mental health referrals system. As the resource, of Woodlands trying to establish itself as a new service, remained the same throughout the chapter (see Chapter 4), I was interested in unpacking the specific aspects of referrals system that influenced how Woodlands operated (see Figure 6 on page 141). From identifying contextual factors of the referrals system, I could then begin to understand what the responses were to Woodlands trying to establish itself as a new service within the referrals system, as well as the subsequent outcomes. These findings were incorporated into a CMOC presented in Chapter 4 (see Figure 7). The $\mathrm{CMOC}$ figure includes several separate $\mathrm{CMOCs}$ relating to the referrals system (see Figure 7). These were incorporated into the same figure to show the influence each $\mathrm{CMOC}$ had on the other CMOCs, which was important to understand how the different contexts influenced service delivery at Woodlands.

In Figure 7, I show that Woodlands, a new inpatient recovery centre, was embedded within an already established mental health system. Woodlands was positioned as a 
recovery centre, but with locked doors, and this appears to be contradictory leading to confusion among referring commissioners. My analysis suggests that the design of Woodlands, and its intended service-user group, did not fit in with the current mental health system or demand for service provision. It is possible that Woodland's long gestation period contributed to this discrepancy and the subsequent events that unfolded concerning securing referrals. As the marketplace has shifted and different needs have been identified, the design and intentions of Woodlands may have been placed on the periphery, or perhaps even outside of what is likely to generate income in the form of referrals. ROC literature is predominately based within community-based and outpatient services (Whitley et al., 2009; Compton et al., 2014), suggesting that the idea of ROC being delivered within inpatient services may be unusual, and may also provide reason as to why Woodlands' approach was on the periphery.

Commissioners categorised the service as a locked rehabilitation service, which differed from how PAs classified it. There were predefined categories for how services are classified on the framework used by commissioners to identify placements. The use of predefined categories was documented in a recent National Collaborative Commissioning Unit position statement in which four 'tiers' of service were identified as being on the Adult Hospital Framework, which are medium secure hospitals, low secure hospitals, controlled egress hospitals (formally known as locked rehabilitation) and uncontrolled egress hospitals (National Collaborative Commissioning Unit, 2020). The findings suggest that there was little flexibility in acknowledging an alternative model of care, meaning Woodlands was compared with the expectations of locked rehabilitation services despite not providing this model of care. This eventually led to Woodlands deviating from the intended service-user group and accepting individuals for whom the service was not originally intended. These findings highlight that the interdependencies of social systems operating at different levels can influence how a service was intended to work and operate, as factors of the referrals system eventually meant the service, at the microsystem level, adapted its inclusion criteria to secure referrals (see Figure 7). Some staff participants articulated concern about this shift to a different clientele by suggesting they do not have the skillset to support these individuals in their recovery. Due to the different classifications of Woodlands, the individuals that commissioners referred 
differed from the intended service-user group (see section 4.5.1.2). At the point of data collection, commissioners were referring individuals with a PD, and/or those who posed a risk, to locked rehabilitation services. The quantitative data available supported the qualitative findings, some service-users rated themselves as having thoughts or behaviours of violence, suicide and self-harm, indicating concerns about safety and harm.

Commissioners are expected to make informed decisions about service-user placements, which includes factors such as location, least restrictive practice and whether an NHS-run service can deliver the treatment, leading commissioners to be cautious about referring service-users to Woodlands (see Figure 7). This resulted in referrals being lower than anticipated and subsequently there being low service-user occupancy at Woodlands throughout the study period. The findings suggest that Woodlands was committed to their model of care and service delivery and this contrasted with how commissioners saw the topography of mental health care delivery. Commissioners appeared wary and uncertain of what Woodlands offered and preferred more tried and tested services that had well-established reputations and whose approaches were evidenced and familiar. A new service with little supporting evidence was a challenge to commissioners' previous experience and they were, on the whole, disinclined to accept assurances based on claims rather than reputation. Nonetheless, commissioners did refer some people to Woodlands and some of these referrals were successful in that people were placed there and received safe care. It is therefore likely that Woodlands will be able to build a reputation over time that will lead to them securing more referrals.

The findings highlight that for a new service, that is not delivered by an established provider, challenges were encountered when trying to launch itself within a competitive and commercial marketplace, which may have been exacerbated by attempting to provide an alternative model of care. Despite Woodlands' good intentions of supporting individuals in their recovery, it appeared that this was not enough to establish itself in the competitive commercial market. Commissioners claimed there was not a great need for a locked, long-term specialist service providing recovery, which could otherwise be provided within the community.

Commissioners also wanted evidence of service effectiveness in the form of quantifiable data, meaning that Woodlands, which was new, and had limited service-user numbers, 
was unable to provide the evidence they wanted. Woodlands was in a paradoxical situation. Commissioners required the service to demonstrate its effectiveness by presenting data on service-user progress, but they were unable to do this as it had not secured enough referrals. This highlights a difficulty for independent organisations when attempting to compete within a commercial market that favours NHS services and more established providers. In addition to this problem, the service appeared to work against its own self-interests. So, for example, despite quantifiable data being needed to inform commissioner decision-making, the routinely collected data at Woodlands was inconsistently gathered and its collection was abruptly terminated. The data that was collected at least shows the potential for charting progress if properly embedded in the design of the service and reliably collated. Incorporating routinely collected data as part of service delivery could be one way the service could provide commissioners with the quantifiable data they need to place individuals at Woodlands.

Participants claimed the effects of low service-user numbers included feeling lonely and lacking a support network, staff feeling demoralised which was associated with staff turnover, and the financial viability of Woodlands being questioned. However, the qualitative data suggested that some service-users liked the limited numbers at Woodlands, suggesting that how individuals feel within group sizes influenced their response to the service's troubles of securing referrals. The challenges of securing referrals influenced staff and service-users on an individual-level, but also the service at the micro-level as its continued existence was questioned by participants. These findings highlight an example of how the EST enabled me to capture the interactions and interdependencies between the different levels of the system in which Woodlands operates.

Woodlands may need to reconsider its criterion, but if it does decide to do this then its approach and design will likely need adjusting. The service needs to be able to flexibly apply its recovery approach, as individuals will have different needs, meaning a one-sizefits delivery is unlikely to work. Although Woodlands' philosophy was for individualised care, some staff were unsure of how they should adapt the recovery approach to meet individual's needs. Staff must be provided with the appropriate support and training to be able to adapt the approach to support the new, unintended service-user group. If 
Woodlands was to accept both subgroups; individuals with a PD, and individuals with severe mental illness, this may be problematic as different types of care would be required within the same setting. However, if Woodlands does not reconsider its intended serviceuser group, and how they can support all persons in their recovery, then the existence of the service may be compromised.

There may be contention over the uniqueness of Woodlands. As outlined in the introduction, there are differences between the term rehabilitation and recovery (see section 1.3.1.2.1), however, Woodlands claimed to be providing something novel and different to other services in the form of recovery, as opposed to rehabilitation. However, these areas of difference could be contested based upon how the service was delivered in practice.

Government policies clearly state that it is the expectation that ALL services provide recovery-oriented care, service-users have autonomy, provide holistic care and focus upon the individual behind the mental illness. Therefore, it could be argued that ALL services have the same intentions as Woodlands, an example of this based upon the findings of this thesis is that Woodlands focused upon changes or skill development within the individual, rather than societal wider issues such as poverty, unemployment and stigma. Whilst it is reasonable to assume that recovery is unlikely to be lived within the confines of services; there was a focus upon developing the skills widely accepted within Western society (e.g. cooking, cleaning) so that service-users could look after themselves in the community. This could be interpreted as a neoliberal view of recovery, which omits the social model of disability view of some service-users (Recovery in the Bin, 2019) (see section 1.3.1.4.1). This raises the question of what role we want our mental health services to have, do we want them to support on an individual level, or do we want them to be agents of social change to ensure wider barriers to individuals living a meaningful and purposeful life are mitigated.

Another area of contention was whether the service provided something different to rehabilitation services which aim to support individuals with complex needs and require more intensive, longer-term care. Findings from a realist synthesis that reviewed a training programme that aimed to support staff who work in mental health rehabilitation 
settings to work in recovery-focused ways generated programme theories that reflect the resources that were identified as important within rehabilitation settings:

- Staff Attitudes - Staff have an understanding that service-users with complex needs have non-linear recovery; but staff do not think they are too hard to engage with in recovery;

- Staff Capacity - Work priorities allow for staff to spend time with service-users on activities;

- Recovery Model - There is a shift from custodial model to recovery-based model, and service users are supported in activities of their choice;

- Service-user Involvement - structures and processes are in place that facilitate and maintain service-user involvement in planning and delivery of the service (e.g. peer mentors, service-user led meetings)

- Positive Risk-Taking - There is a culture where service-user autonomy is encouraged;

- Community - Strong and supportive community and family links (Killaspy et al., 2017).

These resources reflect the values and ambitions of Woodlands, which suggests that these may not be as novel as programme architects at Woodlands articulated. This is likely due to the policy shifts where ROC is the expectation. A reason for this could be that there was a long gestation period before Woodlands was opened - the idea was 25 years old, and the consultations were over 10 years ago. Therefore, at the time of inception these would have been unique and novel ways of working, however in the current policy landscape this appears the expected standard. This may reflect why Woodlands had difficulties in commissioners accepting that they were providing something different to other rehabilitation services.

\section{- To develop transferable theory of wider value to the mental health sector.}

The findings relating to the establishment of a new recovery-oriented service, and how it struggled to deliver upon key principles of ROC can have transferable value for the wider mental health sector. Having considered the findings collectively there appeared to be 
several incongruences, which prevented Woodlands receiving the number of referrals it anticipated and delivering upon its ambition of ROC.

- How Woodlands was classified within the referrals system - recovery versus rehabilitation);

- That Woodlands was providing something unique and could use this uniqueness to distinguish itself from other providers and secure referrals - Woodlands providing ROC versus policies which state that all mental health services must provide ROC;

- Between the evidence Woodlands was able to provide and the evidence that commissioners wanted - anecdotal/awards versus quantified service-user progress and outcomes

- Between the service-users eventually accepted at Woodlands - motivated, ready to work on their recovery and has psychosis versus unmotivated, challenging and risk behaviours and has a personality disorder;

- Between the skillset and training of staff and who was eventually accepted at the service;

- Inconsistencies in how staff worked to support service-users, particularly in their choices and when taking responsibility for DLT (risk adverse vs positive risk-taking)

- Choice being promoted when individuals are within a locked setting and are sectioned under the MHA where there is a power imbalance;

- Adapted Woodlands' practices to avoid rejection/stigma, which limited serviceuser opportunities to establish purpose and a meaningful role within society;

- Inconsistencies in the level of support provided and the abilities/confidence of service-users to engage with resources (e.g. completing DLT or being able to make choices for themselves).

These incongruences negatively influenced the outcomes at Woodlands. Having identified that incongruence was the dominant causal mechanism in how Woodlands struggled to establish itself as a new provider, and in delivering ROC, I sought to identify a substantial theory to provide a conceptual framework. The substantial theory I have identified to explain these findings is congruence model (Nadler \& Tushman, 1980). 


\subsubsection{Congruence Model (Nadler \& Tushman, 1980)}

Nadler and Tushman's (1980, pg. 40) congruence model states the congruence is the degree to which the needs, demands, goals, objectives, and/or structures of one component are consistent with the needs, demands, goals, objectives, and/or structures of another component. In this case one component is Woodlands, and the other component is the referrals system, which is influenced by the wider political and social landscape.

The theory emphasises the critical system characteristic of interdependence, and notes that the congruence of the various components of the system is a necessary condition for organisation effectiveness. If congruence does not exist, which is identified in the findings of this thesis, then consequences such as reduced performance, conflict and dissatisfaction will develop, as seen in the findings of this thesis and outlined in the bullet points above. The theory presents an organisation as a system, which draws various inputs from both internal and external sources, which are transformed into outputs via four chief components - people, work, structure and culture (see Figure 19).

In regards to the important inputs considered within this project I considered the market demands for a service like Woodlands, as well as the current policy landscape to understand the context in which Woodlands was operating. Recovery and ROC now form part of mental health policy, therefore the market demand for a service which claims to be recovery-oriented may not be as high, as all services are required to operate in this way. This shows that the culture and values of Woodlands may not be as unique as PAs believed. Despite this, it shows that the model of care Woodlands was trying to deliver was congruent with the current policy drive. However, there has also been a shift in the service landscape which indicates the community-based care is preferred over inpatient care, meaning those with the greatest needs are accessing inpatient services. The market demand for locked services was to support individuals with risk behaviours such as violence and self-harm, and predominately females with a personality disorder. This was not the same service-user group that Woodlands was designed to support, therefore showing that there was a low fit between the environment of the referrals system (input) and the culture of Woodlands. Although Woodlands adapted its strategy and culture, it did not adapt its people, work or structure to reflect this change in strategy. This resulted 
in several incongruencies which negatively influenced the way in which Woodlands operated and was able to support individuals in their recovery.

- The People - staff lacked the confidence, skills and training to support the individuals who were eventually accepted at the service. Despite staff possessing importance skills such as making people feel accepted, getting to know serviceusers, these skills were not enough for those with more complex or trauma-based backgrounds. This suggests that there was incongruence between the skillset of the staff and what service-users needed from staff. My analysis indicates that although the service adapted its service-user criterion in order to secure referrals it did not ensure that the staffing group available had the necessary knowledge and experiences to meet this need. Additionally, the service-users were not motivated or ready to engage in their recovery or the resources available at Woodlands. Due to this, the support provided by staff did not meet the needs of these individuals. My analysis of research interviews indicates that some staff did not possess the skills, knowledge or ability to adapt their practices to complete the task of supporting these individuals, meaning there was a congruence gap (or low fit) between the task and individuals.

- The Work - There was uncertainty surrounding the tasks that staff were supposed to complete on a daily basis, resulting in inconsistencies within the staffing team. Due to the limited service-user numbers, there were less tasks for staff to complete than anticipated. When these tasks were complete, staff either completed tasks assigned to service-users or did nothing, which meant that the resource of staff was not being maximised efficiently or effectively to support service-users in their recovery. This shows the importance of staff having a clear understanding of what is expected of them, which did not appear forthcoming. Furthermore, staff's ability to complete tasks, such as supporting service-users in their choices, were influenced by stigma and rejection from those in the community, which were beyond the control of the service. This raises the question of whether these tasks will ever be achievable by staff/services unless societal change occurs and what role we want services to play within the mental health 
system. The task of staff to support service-users in their recovery has been informed by neoliberalism, meaning the expectations of staff are to support service-users to become independent, self-sufficient and no longer reliant upon services. The consequence of Woodlands being indirectly, or directly, influenced by recovery policy, which has a neoliberal agenda, is that the culture of delivering recovery as defined by service-users becomes impossible as the focus is on individual service-user change, as opposed to a social model of disability.

- The Structure - Peer mentors also reported that they did not feel they were an established or consulted part of the team and felt that professionally trained staff would make decisions without their input. This hindered the resource of peer mentors and the value of lived experience becoming embedded at Woodlands. This suggests that the structure at Woodlands may not have allowed peer mentors to work as effectively as they could have. This also conflicts with the culture of the service which promotes aims to promote recovery and value the importance of lived experience. Additionally, Woodlands did not have a structure in place to address societal changes needed to fulfil some of its values and vision. This means that the internal structures may support service-users to make choices, but the external structures and the input of societal stigma and rejection into the service prevented the service achieving its strategy of ROC. Blanket rules were adopted by the staffing team which hindered their ability to deliver individualised care which was in keeping with its recovery culture. Lastly, the locked conditions of Woodlands prevented the staff from being able to achieve the culture of recovery where individuals have choice, a meaningful place within society and live their life the way they want to live it.

- The Culture - Woodlands' mission, vision and values related to providing a service that was grounded in recovery. Its purpose was to design and deliver a service based upon service-user defined recovery, as opposed to other conceptualisations of recovery such as clinical recovery. Woodlands positioned this culture as unique and therefore saw this as their unique selling point to establish itself as a new provider. However, when this culture is positioned against the input of 
government policies this vision may not be considered as unique within the mental health system. The operationalisation of ROC at Woodlands could also be considered as neorecovery, which is inconsistent with the vision of promoting service-user recovery that the service set out to achieve but is understandable given its conceptualisation within policy (see section 1.3.1.4.1). Woodlands' recovery approach, which gave responsibility, independence and choice to the service-user, was not considered suitable for individuals with a personality disorder which showed incongruence between the service's culture and the input of who commissioners were referring to locked services.

I have incorporated the findings of this thesis into the organisational congruence model (Nadler \& Tushman, 1980) (see Figure 19). 


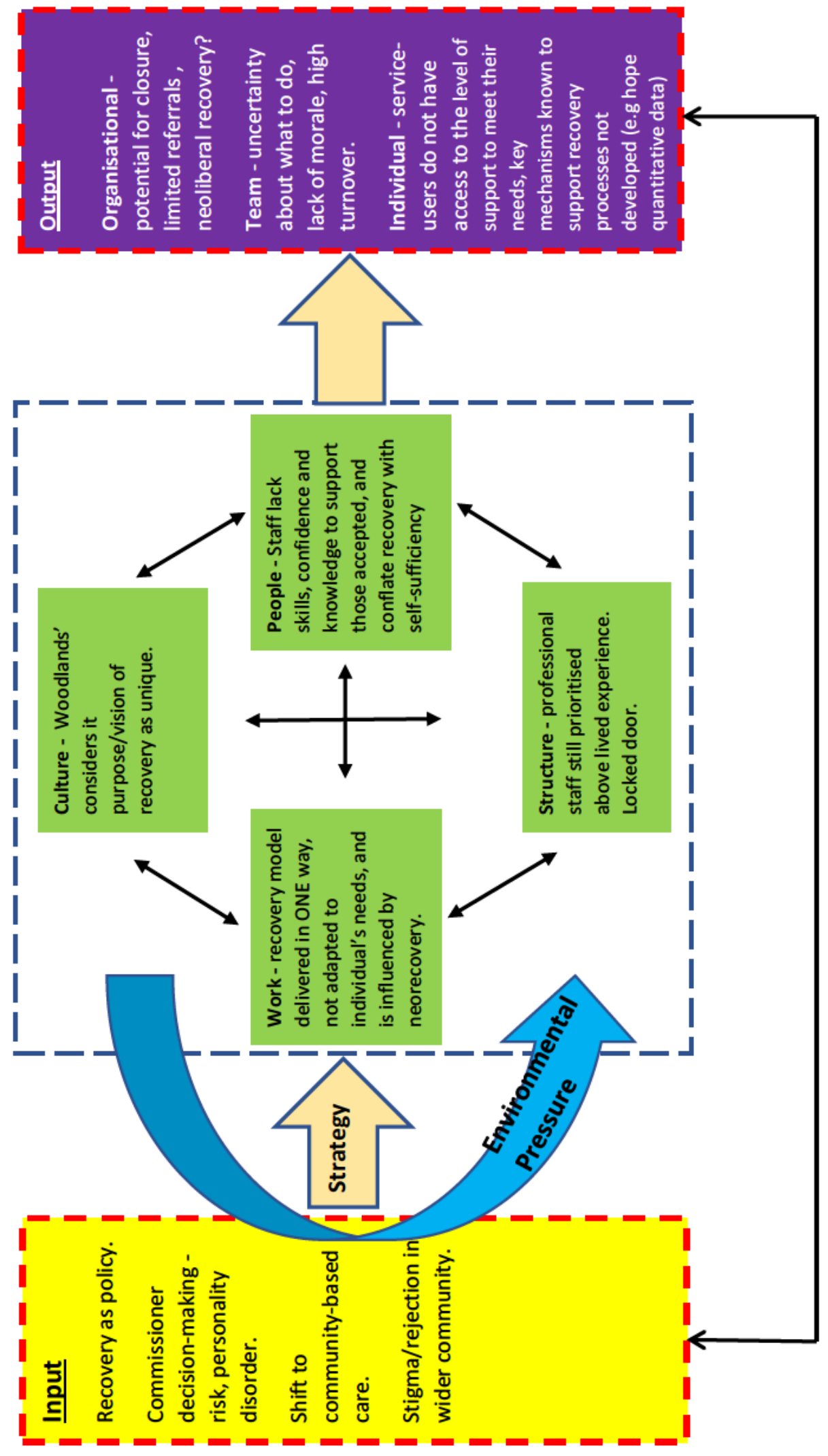

Figure 19 - Proposed Middle-Range Theory (Nasler \& Tushman, 1980)

These findings and the use of organisational congruence model (Nadler \& Tushman, 1980) are translatable for other services who are either struggling to establish themselves as a 
new provider, have adapted their original purpose or are trying to deliver ROC. Being able to identify areas of incongruence is of importance to understand why the organisation, or its value are not being efficiently or effectively delivered. This thesis is the first of its kind, to the author's knowledge, to explore the challenges of a new recovery-oriented inpatient service establishing itself within the UK. The thesis shows the importance of ensuring that the service's strategy aligns with wider environmental inputs. The current policy direction, which is driven by recovery, least restrictive practice, neoliberalism provided a landscape that was not congruent with the culture of Woodlands, therefore it could be argued that struggling to secure referrals was an inevitable eventuality.

If an organisation needs to adapt its original strategy due to environmental inputs, which was the case for Woodlands, then this has ripple effects upon the people, work, culture and structure. Woodlands did not appear to consider the influence upon these facets, which meant there were incongruencies in what Woodlands wanted to provide, and the abilities of those working at the service to be able to provide it. It is therefore imperative that new services are congruent with the current landscape direction and that their strategy and resources reflect this, or else other services are likely to face similar difficulties to what Woodlands encountered.

\subsection{Findings in Relation to Recovery-Oriented Inpatient Care Literature}

The findings of this thesis support the findings reported within the literature review, see Chapter 3. Although in this research project there was not an explicit focus upon how staff conceptualised recovery, several data extracts alluded to a deficit and problem-focused view of recovery, such as staff referring to individuals 'getting better' and service-users needing to change or learn to be able to fit in with society. These articulations, like those presented within other papers on ROC in inpatient contexts (Aston \& Coffey, 2012; Cleary et al., 2013; Coffey et al., 2019a), are clear deviations from service-user definitions of recovery. The findings of this thesis support the findings from the literature review in Chapter 3, where services and staff locate the problem and solution of mental illness with the individual service-user. Aston and Coffey (2012) reported that this could result in 
service-users being held accountable for the success or failure of their recovery. This was reported within the findings of this thesis as in some instances service-users were blamed, or there were potential repercussions, such as referral to more secure services, if serviceusers were unable to live up to the expectations and ideals of the service and recovery.

Hungerford \& Fox (2014) and Coffey et al (2019a) reported that an implication of ROC is that staff adopt a passive and hands-off approach to care. This was supported by the findings of this thesis, which suggested that staff were unsure of how much support should be provided to service-users as this was considered a deviation from recovery.

One finding that did support previous literature was that the context of inpatient settings, which includes the use of restrictions and coercion, prevented ROC being delivered (Chen et al., 2013). It is paradoxical that locked, inpatient mental health services who detain individuals against their will, will be able to provide recovery-oriented environments when there is inequality, a focus upon stabilisation and a need to maintain safety. This raises the question of whether inpatient settings, where individuals are detained in locked conditions, will ever be able to deliver ROC as articulated by service-users (Deegan, 1988; Recovery in the Bin, 2019).

\subsubsection{Original Contribution to Literature}

The findings of this thesis provide several original contributions to literature. Firstly, and most notably, it highlights the processes and challenges of establishing a new recoveryoriented service within the mental health system using congruence model (Nadler \& Tushman, 1980) (see Figure 19). Current ROC literature has only focused upon services that are already established and are shifting their philosophy to be more recovery oriented. In contrast, this study was the first to focus on the establishment and early years of a new service that tried to embed ROC within its model from the outset. The findings of this research captured the difficulties Woodlands faced in securing referrals, and positions these against the policy landscape to understand why these challenges occurred.

I think it is also important and novel to consider the findings of this thesis in relation to recovery as a concept. Whilst I note that recovery as a concept might not have the 
qualities of a theory, it is important to understand the struggles of operationalising recovery in a closed setting such as Woodlands. The original conceptualisations of recovery were based upon the ideas of liberty and freewill, therefore achieving this within the confines of an inpatient setting is contradictory, problematic and arguably impossible. A further issue is that the concept of recovery was never meant originally to be operationalised by services, it was a service-user concept. However, organisations, governments and services such as Woodlands have adopted the notion and incorporated it into frameworks to meet policy needs and forms part of the critique of how recovery has been co-opted and reframed with a neoliberal agenda (Recovery in the Bin, 2019). This thesis shows that the application of recovery by services is problematic, and perhaps what services are really doing is a form of contemporary rehabilitation but dressing it up in the clothes of recovery. My findings highlight that Woodlands encountered a dissonance between what recovery promises and what rehabilitation can deliver, and this lies at the heart of Woodland's issues of delivering ROC. This raises the question of whether it is appropriate or acceptable for services or governments to adopt a serviceuser concept in order to promote their own agenda. Due to the tension surrounding the consensus of 'recovery', any policy or service that has recovery at its centre is precarious and runs the risk of rendering the true meaning and essence of recovery meaningless. The enduring traditions of paternalism and coercion which were evident in the findings of this thesis, regardless of Woodlands' attempt to be patient-centred and offer choice, suggest that the concept of recovery, however it is defined, is unlikely to lead to their elimination, even if the organisation's intentions were well meaning.

Although ROC is considered essential to service delivery within policy, other priorities, such as location and least restrictive practice, are rated more highly. These factors are not inconsistent with ROC but represent that services with a recovery ethos cannot rely upon this philosophy and instead must recognise that commissioners promote ROC in other forms, such as proximity to the persons social networks. These findings are of importance and are an original contribution to the literature as it demonstrates that despite ROC at the forefront of policies in terms of inpatient service delivery, it is prioritised and promoted in a different way by commissioners. Rather than looking at how services individually delivered upon ROC, they used parameters regarding location and social 
support, which is in keeping with the recovery narrative. The findings of this study therefore raise the question of whether commissioners, who are integral to the viability of services, conceptualise or categorise ROC in the same way as how services, such as Woodlands, may attempt to deliver upon this policy. If they do not, then designing an inpatient service with the primary aim of delivering ROC may not be appropriate in the current market and is unlikely to be a unique selling point to distinguish the service from its comparators. If there is a 'low fit' between how the service has been designed in terms of culture, structure, work and people and what commissioners want from inpatient services, then this is likely to result in a limited number of referrals and services needing to adapt their strategy to meet the current policy and market needs. Ultimately, ROC is possible in least restrictive environments, such as independent community living or supported housing, meaning the provision of a locked recovery service is likely to be only appropriate for a very small subset of service-users in a highly competitive market (see section 1.3.3.1 for the prevalence of independent services providing locked rehabilitation).

The unique context of Woodlands provided a focal point for improving 'our' understanding of how contextual layers influenced how the service and its key resources operated in practice (see Figure 20), the words in bold within this figure represent the resources that were considered within this thesis). The findings of this thesis demonstrate the effects contextual layers can have when attempting to introduce a new, recoveryoriented inpatient service into the mental health system (see Figure 20). 


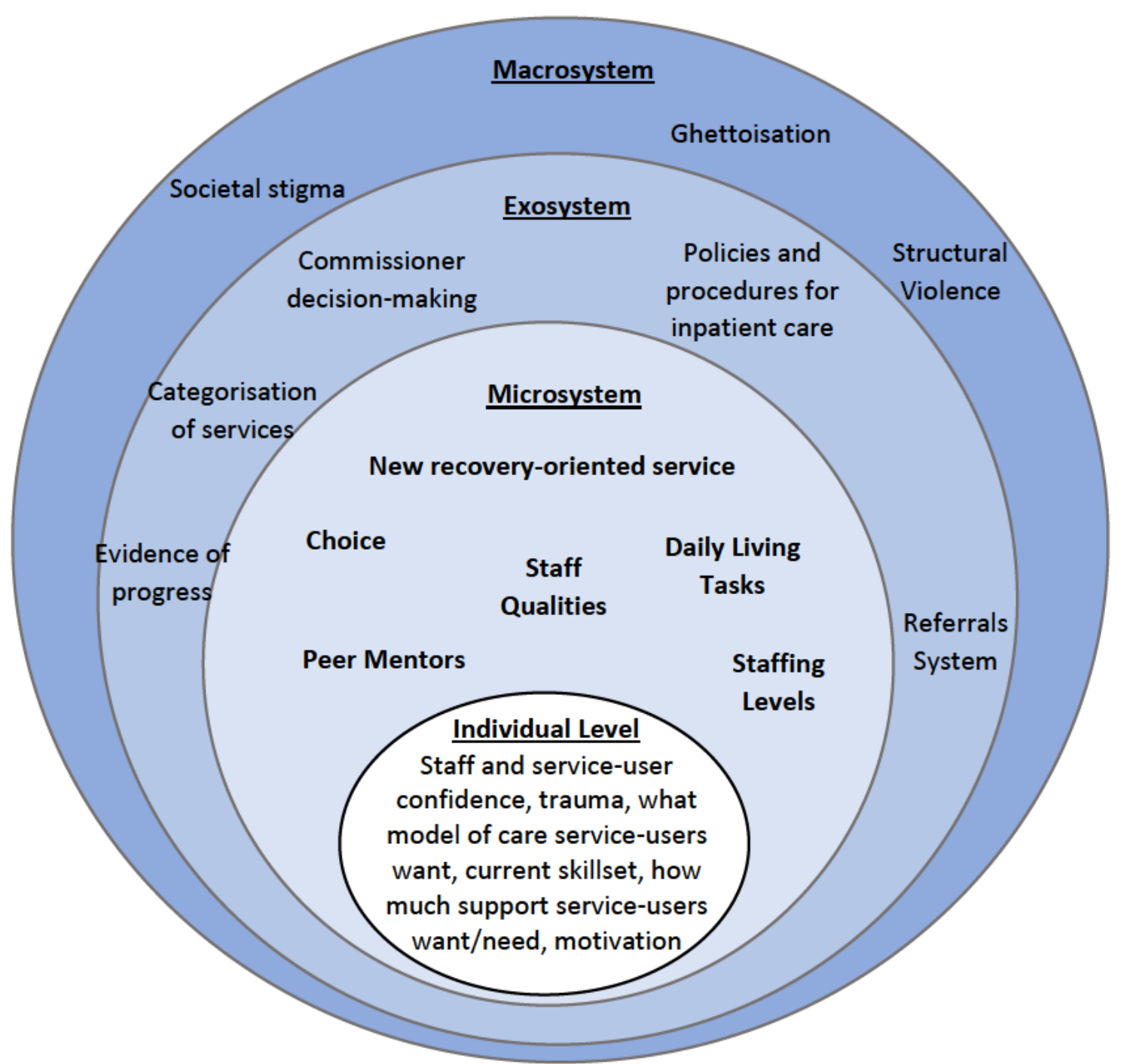

Figure 20 - Using the EST to understand the contextual layers relevant to Woodlands

Lastly, this thesis raises the question of whether mental health services can ever achieve $\mathrm{ROC}$ (as conceptualised within policy) when at the macro-level, processes, such as social exclusion, marginalisation and stigma, continue to influence how mental health services operate. Whilst it is noted that there are individuals who have achieved mental health recovery, despite social systems not always being conducive, it does highlight that even if inpatient services have ambitions to deliver ROC, these may be influenced by social inequalities. Until social change occurs, for some service-users, being equal and participating citizens in society will continue to be rhetoric rather than reality. The findings demonstrate that recoveries may be hampered by negative social experiences and staff may be prompted to limit recovery opportunities to protect service-users from rejection and stigma (see Figure 16). The examples of societal inequalities shared by participants as 
part of this research present unique insights into how inpatient settings can deliver ROC and highlights an important point that policymakers must consider - what role do we want our inpatient services to have within the mental health system, and should this role be to act as agents of social change to address contextual barriers to supporting recovery.

The findings of this thesis show that the challenge of living with a mental illness can be reliant on environmental contingencies. This points to access to independent living, the availability of social support and meaningful daily activity, and relies upon a cycle of increased social capital leading to improved health and secondary benefits of improved confidence and competency in social connections. The availability of community-based resources that facilitate the cycle of increased social capital and its associated benefits, is independent of the actions of Woodlands, or service-users. This means that the implications of stigma and societal prejudice in relation to individuals with mental illhealth is that the cycle to increase social capital and its associated benefits is consistently compromised preventing their recovery. These findings recognise and support the need for social aspects of mental health recovery to be considered within theory and practice.

\subsection{Limitations}

The limitations of this thesis relate to the quantitative data, attribution, the authenticity of accounts and how deviations meant the design changed the focus and outputs of the research.

\subsubsection{Deviation from the Intended Research Project}

The project originally intended to evaluate two interventions, a Physical Health Education Group (PHE) and an Acceptance and Commitment Therapy Group (ACT). The implementation and delivery of these interventions were dependent upon an identified Physical Health Nurse and the Psychologist who were to deliver these approaches. However, due to unforeseen circumstances, these staff members left Woodlands and these interventions were never implemented and delivered.

Despite spending a large part of the PhD designing interview schedules and reviewing relevant literature to develop initial $\mathrm{CMOCs}$, these interventions were unable to be 
evaluated and I had to adapt the research design. This resulted in my decision to evaluate Woodlands as a whole service, meaning considering key components of its philosophy and how the resources available at the service supported individuals in their recovery. This is a slight deviation from typical RE's which focus on an intervention which has parameters and specific resources, whereas, I have used RE principles to evaluate the philosophy and values of a recovery-oriented service. Due to this deviation, and the uniqueness Woodlands being the first inpatient facility run by a charity in the UK, the focus of the research became theory gleaning and development, as opposed to testing and refining theories from literature. In response to being unable to collect data for the PHE and ACT intervention, I also conducted more interviews relating to Woodland's philosophy and was able to include a new participant group, commissioners. This provided me with a rich causal insight into how Woodlands worked within the wider mental health system.

Although the service anticipated that these interventions would be up and running for me to evaluate, this did not occur, showing the complexity of conducting research in realworld settings. From this experience I have learnt the importance of having a Plan B option for research. Based on this experience, I would also recommend to Woodlands that they do not base an entire intervention upon the knowledge or experience of one individual. It also highlights that new services may benefit from knowing what interventions are going to be delivered and how these are going to be achieved before opening and accepting service-users, or else it could result in no interventions being available at the service.

\subsubsection{Not Using A Realist Synthesis to Inform the Development of Initial CMOCs}

I reviewed the resources available relating to ROC in inpatient contexts, as I felt this was the most relevant body of literature that would capture the overall aim of Woodlands to support individuals in their recovery. Due to the scarcity of resources available in this area, I felt that the development of CMOCs would be better achieved through a RE, rather than realist synthesis. Therefore, I focused more of my time and attention upon conducting realist interviews with PAs, staff, service-users and commissioners to develop knowledge of what works, for whom and in what circumstances regarding ROC from the practical 
example of Woodlands. My decision meant that I conducted a review of ROC to identify barriers and facilitators, as opposed to a realist synthesis to develop initial CMOCs that could then be tested at Woodlands. It could be argued that if I reviewed all the resources available for the specific mechanisms that were key to Woodlands' philosophy, I may have achieved more ontological depth and built upon theories previously identified in the literature, rather than starting theory development from scratch.

Due to the challenges I faced, I consulted Dr Justin Jagosh, the Director of the Centre for Advancement in Realist Evaluation and Synthesis (CARES), to advise on how to manage these difficulties. CARES provides education on and promotes the use of realism through training, provides ongoing support for research teams adopting realist evaluation in their projects, and conducts original realist research for health and health-related sciences. Justin advised that, based on the uniqueness of Woodlands, I should instead focus upon developing initial CMOCs specifically from Woodlands which would create new theories about what works, for whom and in what circumstances regarding ROC in inpatient services. My theory development was therefore driven from my understanding of the social architecture of Woodlands, the implicit assumptions about its activity, and how these assumptions played out at Woodlands. This meant I relied upon PAs, staff, serviceusers and commissioners accounts, as opposed to literature. Deriving CMOC development directly from Woodlands also enabled me to capture the unique context of the service, as the UK's first charity-run mental health inpatient facility. This also enabled me to evaluate and develop CMOCs regarding the challenges of establishing a service within the current mental health context, which became a central feature of the project.

Furthermore, as already stated, I had already conducted a realist synthesis of the PHE and ACT intervention intended for Woodlands, however, these interventions were never delivered in practice. Due to this, I also lost the valuable time needed to conduct a realist synthesis which is known to be labour intensive. As the adaption of the research design meant I was evaluating multiple components, resources and values of Woodlands' philosophy, it was hard to condense all these aspects into a realist synthesis that would be manageable and feasible.

In the future, if I were to conduct another RE, I would ensure that the intervention was ready to be delivered, and I fully understood the parameters of the intervention so I could 
also use the resources available to develop initial CMOCs about that intervention under evaluation.

\subsubsection{Limited Quantitative Data Available}

Despite the agreed protocol, the quantitative data was inconsistent and limited by its sample size which meant inferences could not be drawn and I had to rely upon descriptive statistics. There was limited data available for service-users upon admission and therefore a baseline could not determine to note changes over time from the point of admission.

On reflection, it may have been advantageous for me to use the gatekeeper to the service more effectively to ensure that the staff at Woodlands were following the agreed protocol. I used my gatekeeper to establish entry to the service, discuss ethical considerations, understand the day-to-day running of the service and to identify potential participants, however, once data collection began, I did not use this resource as frequently. My rationale for this was to maintain my distance from the service as an independent researcher, but in doing so I lost a potential opportunity for the gatekeeper to ensure the agreed protocol was followed. As she was someone already operating within the delivery of Woodlands, she would have been best placed to oversee this form of data collection, but as I was informed it was routine, I assumed structures were already in place monitoring the collection of this data. In hindsight, I wish I had asked to have monthly access to check the database so I would have had both physical and verbal confirmation that data collection was occurring. This may have also opened up a dialogue between me and the clinical team regarding the decision to cease data collection, and if needs be, I could have taken responsibility for collecting the data, which would have removed my dependency upon the site and its processes.

The quantitative results were based upon data from $43 \%$ of service-users who had accessed Woodlands and therefore I am aware that the data only represents a proportion of service-users. This may mean that the sample may be skewed and therefore biased, for example being more motivated, engaged, or frustrated with the service compared to nonrespondents. Due to this, I acknowledge that different trends may have been identified amongst the service-users who did not complete the outcome measures. Therefore, I have made summations about the people that completed the measures and not 
generalising this to the whole population of service-users who have accessed Woodlands. Despite these limitations, the data still provides some patterns of interest regarding service-users QoL, psychological distress and perception of Woodlands. These potential trends could be used as a guide to help to inform service delivery and to ensure that the service is aligning with its recovery-oriented philosophy.

Woodlands may benefit from discussing with staff and service-users the best way in which routine data could be collected at the service, which is consistent and is built into service delivery. If the routine data collection remains limited this may potentially exacerbate the referrals situation as commissioners want services to quantify service-user progress, which at the point of data collection Woodlands was unable to provide. This highlights the importance of staff being aware of the benefit of routinely data collection and the need for it to be embedded within the organisational structure at Woodlands.

REs recognise that outcomes are different for everyone to some extent due to the individual's capabilities forming part of the context. Due to this the quantitative data was not used to form a $\mathrm{CMOC}$ but was instead used to highlight any convergence, divergence and complementary findings between the quantitative and qualitative datasets. It also enabled me to identify contextual factors, such as service-user and staff attitudes, which added to my understanding of the role of context at Woodlands. Lastly, the data that was collected shows that routine collection of this type of information has promise and could be used internally within the service to learn and focus on improvement. It also has the potential to generate data that will help commissioners in decisions about referrals.

\subsubsection{Carer Perspective Not Captured}

Due to service-user numbers, the potential pool of carers that could be accessed for this project was less than anticipated. As a result, carers were not involved as research participants. Although carers were not the direct recipient of receiving treatment and care at Woodlands, there was an intention for carers to be included as part of the service. Due to this, I considered them secondary recipients and wanted to consider their perspective within the project. I acknowledge that the inclusion of individuals who are not PAs, facilitators or direct recipients of the interventions does not typically reflect other REs, which aims to identify participants who can provide the richest causal insights into what 
works, for whom and in what circumstances (Pawson \& Tilley, 1997). However, it has long been suggested that carers have the potential to contribute and influence service development due to their expertise, experience and knowledge of services (Pearlman \& Holzahausen, 2002). Therefore, I did not want to lose this knowledge, which might lead to unearthing hidden mechanisms, such as service-user responses, as well as outcomes.

In this project, the barrier for carer involvement was service-users not wanting to nominate a carer. Reasons included not having a carer, friend, or loved one, having a difficult relationship with their care, not wanting to burden their carer due to their poor health and fearing consequences of carer involvement about the care they receive at Woodlands. This suggests that there were both structural and relational barriers to recruiting carers as part of this project.

As I required service-users to consent and nominate carers, this highlights the complexity of involving carers in research. I did this to protect the confidentiality and rights of serviceusers as to whether carers are involved in discussions about their care. The recruitment of carers as research participants poses an ethical dilemma on how to access this participant group, whilst also maintaining service-user confidentiality. In future, I may consider using anonymised paper, or online, surveys to access carers, which may help in addressing service-users' concerns regarding the potential repercussions on their care, and may be less of a burden on carer's health problems when compared to the intended face-to-face interview. However, in this project, the most common reason was serviceusers did not have a carer, or had a complicated relationship with their carer. This suggests that mental health services may need to provide support and structures so service-users are able to maintain or work on their relationships with their loved ones, or friends; which is likely to have considerable benefits for individuals in their recovery, but may also indirectly increase the potential pool of carer participants in future research.

In response to being unable to recruit carers for this project, I decided to adapt the research design and recruit commissioners as a new and unintended participant group (see section 2.9). 


\subsubsection{Attribution}

A limit of this project is that it does not claim to draw any conclusions about the effectiveness of the service in supporting service-users in their mental health recovery. The focus was exploratory, seeking to discover and refine theory about the service's causative mechanisms and the circumstances which facilitated or discouraged their activation at the service. The findings do not provide evidence of effect. As a result of taking such an approach, the outcomes accounted for within the research interviews cannot be safely attributed to the service, or its philosophy of care towards choice and responsibility, or the staffing model. The findings presented, therefore, carry a caveat relating to attribution. This is in keeping with RE principles as Pawson (2013, pg. 192) stated "only partial truths emerge from evaluative enquiry"; highlighting that my findings are partial and corrigible and should be subjected to ongoing refinement in future research.

To gain only partial knowledge of Woodlands after extensive time and effort can feel disappointing. However, realists consider that programmes are so complex that no research design would be able to capture a complete picture of every potential influencing feature. RE is, however, more open about the presentation of 'provisional theory' than other epistemologies (Pawson \& Tilley, 1997), which allowed me to develop and refine initial CMOCs about what was, or was not, working, about delivering ROC within a mental health inpatient setting, which can be further tested and refined in future research.

I have highlighted the complexities of delivering service-user-focused recovery within an inpatient context. This may be beneficial in showcasing that by merely placing ROC within mental health policy does not account or overcome the challenges that arise in inpatient contexts and prevent ROC from being delivered within practice. I hope that this RE will pave the way for other researchers to evaluate the delivery of service-user-focused recovery within inpatient care so that we can begin to understand what contexts are conducive for firing the mechanisms needed to support service-user recovery. 


\subsubsection{Authenticity of Accounts}

I am aware that people tell their stories for a particular reason, and seek to engage the listener as they are pulled into the teller's point of view (Reissman, 1993); meaning that caution is needed as narratives need to be viewed as articulating a particular version of themselves and events. This was particularly relevant in this project because of the limited referrals received and accepted at the service and the very real possibility that Woodlands was going to close. Due to the gravity of the situation, such as participants potentially losing their job, I was aware that there was a tension at the time of conducting my interviews and that participants accounts may have been influenced by these circumstances.

In terms of addressing authenticity, I could have considered including elements of ethnography to observe Woodlands operating, and to observe how choice and responsibility, and the staffing model were delivered in practice. This would have presented an opportunity to obtain additional information to understand the theory of change underpinning the Woodlands' philosophy of care in supporting service-users in their recovery. Ethnographic research and realism are both theory-driven and provide detailed and contextualised theoretical accounts of the phenomena in question. Ethnography may therefore offer a complementary method to further understand complex contexts, mechanisms and outcomes.

Although I relied upon participants' interpretations, this does not devalue their insights and experience into what was, and was not, working at Woodlands concerning securing referrals, the staffing model and choice and responsibility, as these offered rich insights in the workings of ROC at Woodlands.

\subsection{Future Research}

Manzano (2016) describes the realist interview as integral to RE and further identifies it as having three phases, namely theory gleaning, refinement and consolidation. Accounts from PAs, staff, service-users and commissioners allowed me to glean and refine theories; however, the resulting CMOCs were not presented to participants as part of the ongoing 
refinement of the CMOCs. Although the findings of the research and subsequent CMOCS formed part of the report disseminated to research participants, I did not ask for responses or hold further interviews to incorporate their views into a further refinement of the CMOCs. A recommendation for future study, therefore, is that the CMOCs are presented to participants, with the aim of further testing and refinement, potential in the form of additional interviews or focus groups.

The current literature and future research relating to ROC may benefit from defining and identifying the parameters of what ROC means in terms of inpatient care. There is a lack of consensus of what ROC means within literature, policy and practice. It may be necessary, based on the findings of this project, for services to consider whether they are delivered upon service-user-focused recovery, or whether neoliberalism has implicitly informed their service delivery. To add to the development of the literature and current evidence-base, which remains in its infancy, further research about the resources of ROC in inpatient contexts, and the mechanisms of change which support recovery would generate a deeper understanding of how recovery-oriented services work to support individuals in their recovery. This could be achieved by further testing of the CMOCs developed in this thesis in other recovery-oriented services. Future research should, therefore, focus on whether recovery-oriented resources are working as intended, what circumstances enable or prevent these resources from working as intended and who these resources work for.

Lastly, barriers to the delivery of service-user-focused recovery within Woodlands, such as stigma, ghettoisation and structural violence, were identified throughout this thesis. All of these barriers impacted upon Woodlands' ability to support service-users in their recovery as intended. The barriers I identified need to be explored and ways to overcome them need to be identified. The insights developed in this thesis could be used to develop more understanding of how, why and in what circumstances services can deliver upon service-user-focused recovery with successful outcomes. 


\subsection{Conclusions}

Pawson $(2006 ; 2013)$ refers to the contextual layers that shape and influence attempts to introduce and sustain new initiatives, such as Woodlands. Contextual layers consist of the individual capacities of the key actors, the interpersonal relationships involved, the institutional setting and wider infrastructural systems; and these can influence attempts to embed interventions into practice (Pawson \& Tilley, 1997).

Having trouble securing and accepting referrals was identified as one of the biggest challenges Woodlands encountered (see Figure 7 on page 141). The contextual layer of the referrals system, factors that influence commissioner's decision-making and the classification of services into predefined categories, became challenging and unexpected contexts in which Woodlands was operating (see Figure 20 on page 273). These unexpectedly challenging contexts negatively influenced Woodlands' ability to secure referrals and establish itself as a new service.

Woodlands eventually accepted individuals with a primary diagnosis of PD and challenging behaviours. This resulted in some staff feeling they did not have the skills, training or confidence to support these individuals in their recovery. If Woodlands is going to continue to accept individuals who do not meet the initial service-user criterion then staff need to be provided with the appropriate resources, so they are able to support all individuals in their recovery. Commissioners appeared to value more tried and tested approaches to inpatient care, which posed challenges for Woodlands trying to establish itself as not only a new provider, but also as a new provider with a different model of care, and meant the service was unable to compete with more established providers. The referrals system was an important contextual layer that participants identified, and some suggested that unless this improves this could cause Woodlands to close (see Figure 7 on page 141).

The resources of the staffing model worked to varying degrees of success, despite serviceusers identifying staff as a valuable part of the service in both the qualitative and quantitative data. The findings show that service-user characteristics and low occupancy were identified as influencing how the staffing model worked in practice. Staff qualities 
were influenced by whether service-users wanted to, and could, form relationships with staff (individual-level), or whether their life experiences, such as trauma, impeded their ability to do this (see Figure 10 on page 153 and Figure 20 on page 273). The trouble of securing referrals affected the 1:2 or 1:2.5 staff to service-user ratios that formed part of the staffing model as there were considerably more staff than service-users (see Figure 12 on page 159). Despite these influences service-users valued positive contact time with staff.

Another influential component was the extent to which appropriate organisational support was put in place to best utilise peer mentors' knowledge and lived experience. PMs needed to be able to distinguish themselves from the rest of the team, and whether service-users were aware of their role. The context in which PMs are embedded within needs to embrace the lived experience of these individuals, so they can differentiate themselves from the other support available at the service. The response to PMs was also shaped by whether service-users were able to see the similarities between themselves and the PMs (individual-level), which influenced whether service-users accessed the lived experience knowledge of PMs (see Figure 14 on page 169). Whilst the liminal identity of PMs was challenging when trying to establish themselves as different to staff, if PMs knowledge and experience was valued by service-users and distinct from other support available, it enabled different ways of working, that professional staff could not achieve, as well as the development of valuable bonds between service-users and staff. This highlighted that the resource of PMs worked in contexts where this form of knowledge was valued by the clinical team and service-users, and when PMs could differentiate themselves from the other forms of support available.

This thesis shows that people building recoveries from mental ill health need support to develop the skills to become independent, and that this support needs to be flexible. The promotion of choice was affected by service-user readiness and their experience of making choices. For service-users who did not have the skills or confidence to make choices of their own they would default back to staff making these choices for them (see Figure 16 on page 191). This highlights a challenge for staff at the service in being able to adapt their approach to choice to meet the needs of each individual service-user. However, despite the need for a flexible approach to choice, some staff members held 
the assumption that all service-users had the ability and skillset needed to make choices and needed to do this without support. The findings showed that this blanket approach to choice was not suitable, and meant that opportunities to learn how to make choices were lost. It is important for staff to find a balance between providing the space to make choices for themselves and providing the support and resources for service-users to be able to engage; and are able to be receptive to each service-user's needs and can adapt their approach accordingly.

Social barriers such as stigma and marginalisation were seen within the findings. These contextual factors, although not part of the immediate environment for service-users, restricted how Woodlands operated. This was shown by staff opting to only use mental health specific opportunities to protect service-users from further discrimination and rejection (see Figure 16 on page 191 and Figure 20 on page 273). Although the immediate environment may support and advocate choice and independence, this was not reflective of all the environments service-users engaged within. The challenge Woodlands faced was attempting to support service-users to be part of their community, where stigma remains an ongoing social problem. These findings show that inpatient mental health settings cannot be viewed as operating within isolation as social processes, that are beyond the control of Woodlands, can negatively influence individual's recovery.

The final resource considered in this thesis was the expectation that DLT would be completed by service-users. For service-users with little to no experience and confidence, there were concerns that they would be judged and therefore they avoided engaging in such tasks (see Figure 18 on page 200). For staff who lacked confidence in completing the DLT, they did not encourage or provide service-users with the support and motivation needed to engage, whereas other staff members who were confident proactively engaged others and encouraged others to partake. There was variation in how this resource was delivered which resulted in differing outcomes of service-user participation. Inpatient services are also governed by policies and procedures (see Figure 18 on page 200 and Figure 20 on page 273), which was a source of frustration for service-users as it prevented them from easily accessing the equipment needed to complete the DLT. These contextual layers, as well as the service-user responses to the resource meant that staff often 
completed the DLT, meaning service-users missed out on opportunities to develop the skills PAs identified as necessary for independent living.

The overall research question for this thesis was:

What works, for whom and in what circumstances at Woodlands in supporting serviceuser recovery?

It appears from the findings presented that Woodlands encountered several challenges when trying to establish itself as a new service within the mental health referrals system. This meant that Woodlands was not working as anticipated as it was operating at lower numbers than expected. The circumstances that prevented Woodlands from working as intended largely centred on factors influencing commissioners' decision-making which limited the potential pool of service-users referred, and the fact the service could not provide the evidence needed to compete with tried and tested providers. This had ripple effects on how Woodlands delivered its recovery model, for example in terms of the staff to service-user ratios (see Figure 12 on page 159), and staff deviating from the expected service delivery by completing DLT on service-users behalf (see Figure 18 on page 200).

Woodlands appeared to work best for individuals who made what staff saw as the right decisions, were ready to accept responsibility for their recovery and could imagine living a life within the community. However, this might be due to the fact this was PAs vision of who Woodlands would support, and staff may have felt more comfortable in supporting these individuals in their recovery. The findings of this thesis raise the question of whether the individuals that Woodlands is considered to work best for are likely to be referred to a specialist, private locked service in the current market of least restrictive practice and less outsourcing of care to private providers. If Woodlands does not consider this question, then the problem of referrals is likely to persist and could result in the closure of the service.

The findings of this thesis show the interrelationships between the different levels of the system and how the context of Woodlands shaped the way the service operated. These findings are informative, transferable and identify important issues that need to be 
addressed academically, politically and within practice. It also highlights that an ecological lens within mental health may be advantageous to capture the complexity of mental health recovery and service delivery. In terms of the impact of this thesis, the research highlights the importance of context when establishing a new, recovery-oriented mental health service. These findings will be of direct relevance to mental health service providers, service-users, policymakers and academics to ensure we are reminded that mental health services do not operate in isolation and are part of a wider system that needs to work together to ensure we are delivering upon the promise and vision of ROC. But arguably most importantly, these findings raise the question of whether ROC, as defined by service-users, will ever be able to be achieved within inpatient settings - and if this is an impossible task, then where does recovery-oriented policies go from here? 


\section{REFERENCES}

Aday, L. A., \& Cornelius, L. J. (2006). Designing and conducting health surveys: a comprehensive guide. John Wiley \& Sons.

Ådnøy Eriksen, K., Arman, M., Davidson, L., Sundfør, B., \& Karlsson, B. (2014). Challenges in relating to mental health professionals: Perspectives of persons with severe mental illness. International Journal of Mental Health Nursing, 23(2), 110-117.

Akinci, F., Esatoglu, A. E., Tengilimoglu, D., \& Parsons, A. (2005). Hospital choice factors: a case study in Turkey. Health Marketing Quarterly, 22(1), 3-19.

Altheide, D. L., \& Johnson, J. M. (2011). Reflections on interpretive adequacy in qualitative research. The SAGE handbook of qualitative research, 4, 581-594.

Altman, D. G., Schulz, K. F., Moher, D., Egger, M., Davidoff, F., Elbourne, D., \& Lang, T. (2001). The revised CONSORT statement for reporting randomized trials: explanation and elaboration. Annals of internal medicine, 134(8), 663-694.

Altrichter, H., Posch, P., \& Somekh, B. (1996). Teachers investigate their work: An introduction to the methods of action research. Psychology Press.

Andresen, R., Oades, L., \& Caputi, P. (2003). The experience of recovery from schizophrenia: towards an empirically validated stage model. Australian \& New Zealand Journal of Psychiatry, 37(5), 586-594.

Anthony, W. A (1993) Recovery from mental illness: The guiding vision of the mental health system in the 1990s. Psychosocial Rehabilitation Journal, 16(4), 11-23.

Anzul, M., Downing, M., Ely, M., \& Vinz, R. (2003). On writing qualitative research: Living by words. Routledge.

Appelbaum, P. S., \& Grisso, T. (2001). MacArthur competence assessment tool for clinical research (MacCAT-CR). professional resource press/professional resource exchange.

Aschbrenner, K. A., Mueser, K. T., Bartels, S. J., \& Pratt, S. I. (2013). Perceived social support for diet and exercise among persons with serious mental illness enrolled in a healthy lifestyle intervention. Psychiatric Rehabilitation Journal, 36(2), 65.

Askey, R., Holmshaw, J., Gamble, C., \& Gray, R. (2009). What do carers of people with psychosis need from mental health services? Exploring the views of carers, service users and professionals. Journal of Family Therapy, 31(3), 310-331.

Astbury, B. (2013). Some reflections on Pawson's Science of Evaluation: A Realist Manifesto. Evaluation 19. (4). 383-401.

Aston, V., \& Coffey, M. (2012). Recovery: what mental health nurses and service users say about the concept of recovery. Journal of Psychiatric and Mental Health Nursing, 19(3), 257-263.

Arnett, J. J. (1995). Broad and narrow socialization: The family in the context of a cultural theory. Journal of Marriage and the Family, 617-628. 
Australian Government. (2009). Fourth National Mental Health Plan: An Agenda for Collaborative Government Action in Mental Health 2009-2014. Canberra: Commonwealth of Australia.

Aylward, M., Phillips, C. \& Howson, H. (2013). Simply Prudent Healthcare - achieving better care and value for money in Wales - discussion paper. Cardiff: The Bevan Commission.

Bachrach, L. L. (1997). Lessons from the American experience in providing communitybased services. Care in the community: illusion or reality, 21-36.

Baumgartner, J. N., \& Burns, J. K. (2014). Measuring social inclusion-a key outcome in global mental health. International journal of epidemiology, 43(2), 354-364.

Bazeley, P., \& Jackson, K. (Eds.). (2013). Qualitative data analysis with NVivo. Sage Publications Limited.

Beck, A. T., Epstein, N., Brown, G., \& Steer, R. A. (1988). An inventory for measuring clinical anxiety: psychometric properties. Journal of consulting and clinical psychology, 56(6), 893.

Beitinger, R., Kissling, W., \& Hamann, J. (2014). Trends and perspectives of shared decision-making in schizophrenia and related disorders. Current Opinion in Psychiatry, 27(3), 222-229.

Bell, E., (2014). Reconfiguring the state under the Coalition: Shoring up state power through the Big Society. Observatoire de la société britannique, (15), 129-144.

Berardelli, I., Sarubbi, S., Rogante, E., Hawkins, M., Cocco, G., Erbuto, D., \& Pompili, M. (2019). The Role of Demoralization and Hopelessness in Suicide Risk in Schizophrenia: A Review of the Literature. Medicina, 55(5), 200.

Beresford, P. (2002) 'Thinking about "mental health": Towards a social model', Journal of Mental Health, 11(6), $581-4$.

Bergman, M. (2008). The Straw Men of the Qualitative-Quantitative Divide and their Influence on Mixed Methods Research. Advances in Mixed Methods Research: Theories and Applications. Bergman, M. London, Sage Publications.

Bhaskar, R. (1975). A realist theory of science. Leeds: Leeds Book.

Bhaskar, R. (2008) A Realist Theory of Science. London: Routledge.

Blanc, X., Collet, T., Auer, R., Fischer, R., Locatelli, I., Iriarte, P., Cornuz, J. (2014). Publication trends of shared decision making in 15 high impact medical journals: A full text review with bibliometric analysis. BMC Medical Informatics and Decision Making, 14(1), 1.

Blaxter, M. (2010) Health. (2nd ed), Cambridge: Polity Press.

Borg, M., \& Kristiansen, K. (2004). Recovery-oriented professionals: Helping relationships in mental health services. Journal of mental health, 13(5), 493-505. 
Borg, M., Sells, D., Topor, T., Mezzina, R., Marin, I., \& Davidson, L. (2005). What Makes a House a Home: The Role of Material Resources in Recovery from Severe Mental Illness. American Journal of Psychiatric Rehabilitation, 8, 243- 256.

Bowers, L., Carr-Walker, P., Allan, T., Callaghan, P., Nijman, H., \& Paton, J. (2006). Attitude to personality disorder among prison officers working in a dangerous and severe personality disorder unit. International Journal of Law and Psychiatry, 29(5), 333-342.

Braslow, J. T. (2013). The manufacture of recovery. Annual review of clinical psychology, 9 , 781-809.

Braun, V., \& Clarke, V. (2006). Using thematic analysis in psychology. Qualitative research in psychology, 3(2), 77-101.

Brijnath, B., \& Antoniades, J. (2016). "I'm running my depression:" Self-management of depression in neoliberal Australia. Social Science \& Medicine, 152, 1-8.

Brett, J. O., Staniszewska, S., Mockford, C., Herron-Marx, S., Hughes, J., Tysall, C., \& Suleman, R. (2014). Mapping the impact of patient and public involvement on health and social care research: a systematic review. Health Expectations, 17(5), 637-650.

Brohan, E., Elgie, R., Sartorius, N., Thornicroft, G., \& GAMIAN-Europe Study Group. (2010). Self-stigma, empowerment and perceived discrimination among people with schizophrenia in 14 European countries: the GAMIAN-Europe study. Schizophrenia research, 122(1-3), 232-238.

Bronfenbrenner, U. (1975). Reality and research in the ecology of human development. Proceedings of the American Philosophical Society, 119(6), 439-469.

Bronfenbrenner, U. (1977). Toward an experimental ecology of human development. American psychologist, 32(7), 513.

Bronfenbrenner, U. (1978). The social role of the child in ecological perspective. Journal of Sociology, 7 (1), 4-20.

Byng, R., Norman, I. \& Redfern, S. (2005) Using realistic evaluation to evaluate a practice level intervention to improve primary healthcare for people with long-term mental illness. Evaluation. 11. 69-93.

Byrne, M. K., Sullivan, N. L. \& Elsom, S. (2007). Clinician optimism: Development and psychometric analysis of a scale for mental health clinicians. Australian Journal of Rehabilitation Counselling, 12, 11-20.

Cahill, J., Paley, G., \& Hardy, G. (2013) What do patients find helpful in psychotherapy? Implications for the therapeutic relationship in mental health nursing. Journal of Psychiatric and Mental Health Nursing, 20(9), 782-791.

Charles, C., Gafni, A., \& Whelan, T. (1997). Shared decision-making in the medical encounter: what does it mean?(or it takes at least two to tango). Social science \& medicine, 44(5), 681-692. 
Charlton, J. I. (2000). Nothing about us without us: Disability oppression and empowerment. Univ of California Press.

Chin, M. H., Kirchhoff, A. C., Schlotthauer, A. E., Graber, J. E., Brown, S. E., Rimington, A., \& Shook, M. E. (2008). Sustaining quality improvement in community health centers: perceptions of leaders and staff. The Journal of ambulatory care management, 31(4), 319.

Chen, D., Liu, S., Kingsbury, P., Sohn, S., Storlie, C. B., Habermann, E. B., \& Liu, H. (2019). Deep learning and alternative learning strategies for retrospective real-world clinical data. NPJ digital medicine, 2(1), 1-5.

Chen, S. P., Krupa, T., Lysaght, R., McCay, E., \& Piat, M. (2013). The development of recovery competencies for in-patient mental health providers working with people with serious mental illness. Administration and Policy in Mental Health and Mental Health Services Research, 40(2), 96-116

Chisholm, J., \& Petrakis, M. (2020). Peer Worker Perspectives on Their Potential Role in the Success of Implementing Recovery-Oriented Practice in a Clinical Mental Health Setting. Journal of Evidence-Based Social Work, 1-17.

Chow, W. S., \& Priebe, S. (2013). Understanding psychiatric institutionalization: a conceptual review. BMC psychiatry, 13(1), 169.

Cleary, M., Hunt, G.E., Horsfall, J., Deacon, M. (2012). Nurse-Patient Interaction in Acute Adult Inpatient Mental Health Units: a Review and Synthesis of Qualitative Studies, Issues in Mental Health Nursing, 33(2), 66-79.

Cleary, M., Horsfall, J., O'Hara-Aarons, M., \& Hunt, G. E. (2013). Mental health nurses' views of recovery within an acute setting. International Journal of Mental Health Nursing, 22(3), 205-212.

Coffey, M., Hannigan, B., Barlow, S., Cartwright, M., Cohen, R., Faulkner, A., \& Simpson, A. (2019a). Recovery-focused mental health care planning and co-ordination in acute inpatient mental health settings: a cross national comparative mixed methods study. $B M C$ psychiatry, 19(1), 115.

Coffey, M., Hannigan, B., Meudell, A., Jones, M., Hunt, J., \& Fitzsimmons, D. (2019b). Quality of life, recovery and decision-making: a mixed methods study of mental health recovery in social care. Social psychiatry and psychiatric epidemiology, 54(6), 715-723.

Cohen, L., \& Manion, L. (1986). Research methods in education. Routledge. London: Croom Helm.

Cohen, L., Manion, L. and Morrison, K. (2010). Research Methods in Education. London and New York: Routledge.

Compte, M. D. L., \& Goetz, J. P. (1982). Problems of reliability and validity in ethnographic research. Review of educational research, 52(1), 31-60.

Compton, M. T., Reed, T., \& Broussard, B. (2014). Development, implementation, and preliminary evaluation of a recovery-based curriculum for community navigation 
specialists working with individuals with serious mental illnesses and repeated hospitalizations. Community Mental Health Journal, 50, 383-387.

Cornuz, J., Kuenzi, B., \& Krones, T. (2011). Shared decision making development in Switzerland: room for improvement. Z Evid Fortbild Qual Gesundhwes, 105(4), 296-299.

Corrigan, P. (2004). How stigma interferes with mental health care. American psychologist, 59(7), 614.

Corrigan, P. W., \& Phelan, S. M. (2004). Social support and recovery in people with serious mental illnesses. Community mental health journal, 40(6), 513-523.

Corrigan, P. W., \& Rao, D. (2012). On the self-stigma of mental illness: Stages, disclosure, and strategies for change. The Canadian Journal of Psychiatry, 57(8), 464-469.

Coulter, A., \& Collins, A. (2011). Making shared decision-making a reality: no decision about me, without me. King's Fund.

Cox, A., Hayter, M., \& Ruane, J. (2010). Alternative approaches to 'enhanced observations' in acute inpatient mental health care: a review of the literature. Journal of psychiatric and mental health nursing, 17(2), 162-171.

Cradock, G. (2007). The responsibility dance: Creating neoliberal children. Childhood, 14(2), 153-172

Cree, L., Brooks, H. L., Berzins, K., Fraser, C., Lovell, K., \& Bee, P. (2015). Carers' experiences of involvement in care planning: a qualitative exploration of the facilitators and barriers to engagement with mental health services. BMC psychiatry, 15(1), 208.

Creswell, J. W., \& Clark, V. L. P. (2017). Designing and conducting mixed methods research. Sage publications.

Critical Appraisal Skills Programme. (2014). CASP checklists. Critical Appraisal Skills Programme (CASP): Making sense of evidence. Retrieved $20^{\text {th }}$ March 2020, from http://www.casp-uk.net/appraising-the-evidence.

Crowhurst, I. (2013). The fallacy of the instrumental gate? Contextualising the process of gaining access through gatekeepers. International Journal of Social Research Methodology, 16(6), 463-475.

Currid, T. J., Turner, A., Bellefontaine, N. \& Spada, M. M. (2012). Mental health issues in primary care: implementing policies in practice. British Journal of Community Nursing, 17(1), 21-26.

Dalkin, S., Greenhalgh, J., Jones, D., Cunningham B. \& Lhussier M. (2015). What's in a mechanism? Implementation Science. 10.(49).

Davidson, L., Borg, M., Marin, I., Topor, A., Mezzina, R., \& Sells, D. (2005). Process of recovery in mental illness: Findings from a multinational study. American journal of Psychiatric Rehabilitation, 8, 177-201. 
Davidson, L. E., Harding, C. E., \& Spaniol, L. E. (2005). Recovery from severe mental illnesses: Research evidence and implications for practice, 1. Centre for Psychiatric Rehabilitation/Boston U.

Davidson, L., Chinman, M., Sells, D., \& Rowe, M. (2006). Peer support among adults with serious mental illness: a report from the field. Schizophrenia bulletin, 32(3), 443-450.

Davidson, L., \& Roe, D. (2007). Recovery from versus recovery in serious mental illness: One strategy for lessening confusion plaguing recovery. Journal of Mental Health, 16(4), 459-470.

Davidson, L., Rowe, M., Tondora, J., O'Connell, M. J., \& Lawless, M. S. (2008). A practical guide to recovery-oriented practice: Tools for transforming mental health care. Oxford University Press.

Davidson, L., Drake, R. E., Schmutte, T., Dinzeo, T., \& Andres-Hyman, R. (2009). Oil and water or oil and vinegar? Evidence-based medicine meets recovery. Community mental health journal, 45(5), 323-332.

Davidson, L., \& Chan, K. K. (2014). Common factors: evidence-based practice and recovery. Psychiatr Serv, 65. 675-677.

Davidson, L., Carr, E., Bellamy, C., Tondora, J., Fossey, E., Styron, T., \& Elsamra, S. (2016). Principles for recovery-oriented inpatient care. In Handbook of recovery in inpatient psychiatry, 39-58. Springer, Cham.

Deegan, M. J. (1989). American ritual dramas: Social rules and cultural meanings. Greenwood Publishing Group.

Deegan, P. E. (1988). Recovery: The lived experience of rehabilitation. Psychosocial rehabilitation journal, 11(4), 11.

Deegan, P. (2001). Recovery: The Lived Experience of Rehabilitation. Psychological and Social Aspects of Psychiatric Disability. Centre for Psychiatric Rehabilitation. Sargent College of Health and Rehabilitation Sciences. Boston University. Boston, 92-98.

Deegan, P. E. (2002). Recovery as a self-directed process of healing and transformation. Occupational Therapy in Mental Health, 17(3-4), 5-21.

Deegan, P. E., \& Drake, R. E. (2006). Shared decision making and medication management in the recovery process. Psychiatric Services (Washington, D.C.), 57(11), 1636-1639.

Denscombe, M. (2010). The Good Research Guide: for small-scale social research. McGraw Hill.

Denzin, N.K. (1970). The research act: A theoretical introduction to sociological methods. Chicago: Aldine.

Denzin, N.K. (1978). Sociological methods: A sourcebook. New York, NY: McGraw-Hill.

Denzin, N. K. (1994). Introduction: Entering the field of qualitative research. Handbook of qualitative research. 
Denzin, N. K. (2009). The research act: A theoretical introduction to sociological methods (3rd ed.). Englewood Cliffs, NJ: Prentice Hall.

Denzin, N.K., \& Lincoln, Y.S. (2005). Introduction: The discipline and practice of qualitative research'. In Denzin, N.K., \& Lincoln, Y.S. (Eds.), The Sage handbook of qualitative research, 1-32. Thousand Oaks, CA: Sage.

De Laine, M. (2000). Fieldwork, participation and practice: Ethics and dilemmas in qualitative research. London, United Kingdom: Sage.

Delaney, K. R. (2012). Moving to a recovery framework of care: focusing attention on process. Archives of psychiatric nursing, 26(2), 165-166.

Dennison, S. (2010). Peer mentoring: Untapped potential. Journal of Nursing Education, 49(6), 340-342.

Department of Health. (2001) The Journey to Recovery- The Governments Vision for Mental Health Care. London.

Department of Health. (2006) Our Health, Our Care, Our Say. London.

Department of Health. (2007) Best practice in managing risk: principles and guidance for best practice in the assessment and management of risk to self and others in mental health services: London.

Department of Health. (2008a) High Quality Care for All. London.

Department of Health. (2008b) Refocusing the CPA: Policy and Positive Practice Guidance. London.

Department of Health. (2011) No health without mental health: a cross-government mental health outcomes strategy for people of all ages. Crown Copyright: London.

Department of Health. (2015). Mental Health Act 1983: Code of Practice.

Department of Health and Ageing. (2009). Fourth national mental health plan: an agenda for collaborative government action in mental health 2009-2014. Canberra: Commonwealth of Australia.

Department of Health Social Services and Public Safety (Northern Ireland). (2010) Service framework for mental health and wellbeing. Belfast: Department of Health Social Services and Public Safety.

Derogatis, L. R. (1992). SCL-90-R: Administration, scoring \& procedures manual-II for the (revised) version and other instruments of the psychopathology rating scale series. Clinical Psychometric Research., 1-16.

De Ruysscher, C., Vandevelde, S., Tomlinson, P., \& Vanheule, S. (2020). A qualitative exploration of service users' and staff members' perspectives on the roles of inpatient settings in mental health recovery. International Journal of Mental Health Systems, 14(1).

Dierdorff, E. C., \& Morgeson, F. P. (2013). Getting what the occupation gives: Exploring multilevel links between work design and occupational values. Personnel Psychology, 66(3), 687-721. 
Dijs-Elsinga, J., Otten, W., Versluijs, M. M., Smeets, H. J., Kievit, J., Vree, R., \& Marang-van de Mental healtheen, P. J. (2010). Choosing a hospital for surgery: the importance of information on quality of care. Medical Decision Making, 30(5), 544-555.

Disability Rights Commission. (2007). Independent living and the Commission for Equality and Human Rights. London: Disability Rights Commission.

Donald, F., Duff, C., Lee, S., Kroschel, J. \& Kulkarni, J.(2015). Consumer perspectives on the therapeutic value of a psychiatric environment. Journal of Mental Health, 24, 63-67.

Durocher, E., Gibson, B. E., \& Rappolt, S. (2014). Occupational justice: A conceptual review. Journal of Occupational Science, 21(4), 418-430.

Emmel, N., Hughes, K., Greenhalgh, J., \& Sales, A. (2007). Accessing socially excluded people-Trust and the gatekeeper in the researcher-participant relationship. Sociological Research Online, 12(2), 1-13.

Engel, G. L. (1977). The need for a new medical model: a challenge for biomedicine. Science, 196(4286), 129-136.

English Oxford Living Dictionaries (2018), "To ghettoize", Oxford University Press, available at: https://en.ox forddictionaries.com/definition/ghettoize (accessed $2^{\text {nd }}$ March, 2020).

Etheridge, F., Couturier, Y., Denis, J. L., Tremblay, L., \& Tannenbaum, C. (2014). Explaining the success or failure of quality improvement initiatives in long-term care organizations from a dynamic perspective. Journal of Applied Gerontology, 33(6), 672-689.

Etherington, K. (2004). Becoming a reflexive researcher using our selves in research. London: Jessica Kingsley Publishers.

Evans, C., Connell, J., Barkham, M., Margison, F., McGrath, G., Mellor-Clark, J., \& Audin, K. (2002). Towards a standardised brief outcome measure: Psychometric properties and utility of the CORE-OM. The British Journal of Psychiatry, 180(1), 51-60.

Evans, D., \& Killoran, A. (2000). Tackling health inequalities through partnership working: learning from a realistic evaluation. Critical Public Health, 10(2), 125-140.

Evans, J., Rose, D., Flach, C., Csipke, E., Glossop, H., Mccrone, P., \& Wykes, T. (2012). VOICE: developing a new measure of service users' perceptions of inpatient care, using a participatory methodology. Journal of Mental Health, 21(1), 57-71.

Evans, R., \& Collins, H. M. (2007). Rethinking expertise. University of Chicago Press.

Ewbank, L., Thompson, J., \& McKenna, H. (2017). NHS hospital bed numbers: past, present, future. Kings Fund. Available at: https://www.kingsfund.org.uk/publications/nhs-hospital-bed-numbers (accessed on 3rd July 2020).

Fakhoury, W., \& Priebe, S. (2007). Deinstitutionalization and reinstitutionalization: major changes in the provision of mental healthcare. Psychiatry, 6(8), 313-316.

Farber, B. A. (2006). Self-disclosure in psychotherapy. Guilford Press. 
Farmer, P. (2004). An Anthropology of Structural Violence. Current Anthropology, 45(3), 305- 317.

Farmer, T., Robinson, K., Elliott, S. J., \& Eyles, J. (2006). Developing and implementing a triangulation protocol for qualitative health research. Qualitative health research, 16(3), 377-394.

Farkas, M. (2007). The vision of recovery today: what it is and what it means for services. World Psychiatry, 6(2), 68.

Farkas, M., Gagne, C., Anthony, W., \& Chamberlin, J. (2005). Implementing recoveryoriented evidence-based programs: Identifying the critical dimensions. Community mental health journal, 41(2), 141-158.

Fielding, N.G., \& Fielding, J.L. (1986). Linking data (Vol. 4). Beverly Hills, CA: Sage.

Foucault, M. (1985). The use of pleasure. New York: Pantheon Books.

Foucault, M. (2008). Psychiatric power: Lectures at the college de France, 1973--1974. Macmillan.

Foye, U., Li, Y., Birken, M., Parle, K., \& Simpson, A. (2020). Activities on acute mental health inpatient wards: a narrative synthesis of the service users' perspective. Journal of Psychiatric and Mental Health Nursing.

Frank, R. \& Glied, S. (2006) Better but not well: Mental Health Policy in the United States since 1950. Baltimore: The John Hopkins University Press.

Fritzsche, K., Monsalve, S. D., Abbo, C., \& Frahm, G. (2014). The doctor-patient relationship. In Psychosomatic Medicine (pp. 25-31). Springer, New York, NY.

Gale, J., \& Marshall-Lucette, S. (2012). Community mental health nurses' perspectives of recovery-oriented practice. Journal of psychiatric and mental health nursing, 19(4), 348353.

Gibson, D., Gonzales, J. L., \& Castanon, J. (2006). The importance of reputation and the role of public relations. Public relations quarterly, 51(3), 15.

Gilburt, H., Rose, D., \& Slade, M. (2008). The importance of relationships in mental health care: A qualitative study of service users' experiences of psychiatric hospital admission in the UK. BMC health services research, 8(1), 92.

Gillard, S., Gibson, S. L., Holley, J., \& Lucock, M. (2015). Developing a change model for peer worker interventions in mental health services: a qualitative research study. Epidemiology and Psychiatric Sciences, 24(5), 435-445.

Gillespie, M., \& Clarke, A. (2007). Recovery group for people with severe and enduring mental health problems. Clinical Psychology Forum-New Series, 172, 15.

Gillham, B. (2005). Research Interviewing: The Range of Techniques. Berkshire: McGrawHill Education.

Glasby, J. \& Tew, J. (2015). Mental health policy and practice (3rd ed.). London: Palgrave Macmillan. 
Gofal. (2015). Snapshot 4: People's experiences of primary mental health services in Wales - Three years on. Bridgend: Gofal.

Goffman, E. (1961). Encounters: Two studies in the sociology of interaction. Ravenio Books.

Goldberg, D.P. (1972). The detection of psychiatric illness by questionnaire. Maudsley Monograph. 21. Oxford: Oxford University Press.

Greenhalgh, T., Wong, G., Jagosh, J., Greenhalgh, J., Manzano, A., Westhorp, G., \& Pawson, R. (2015). Protocol-the RAMESES II study: developing guidance and reporting standards for realist evaluation. BMJ open, 5(8).

Greenhalgh, T., Greenhalgh, J., Pawson., R., Manzano, A., Wong, G., Jagosh, J., Westhorp, G. (2017). Retroduction in realist evaluation. The RAMESES II Project. Viewed $3^{\text {rd }}$ October 2019 https://www.ramesesproject.org/media/RAMESES_II_Retroduction.pdf

Greenhalgh, T., Greenhalgh, J., Pawson., R., Manzano, A., Wong, G., Jagosh, J., Westhorp, G. (2017). Quality Standards for Realist Evaluation. For Evaluators and Peer-reviewers. The $\begin{array}{lllll}\text { Rameses II Project. Viewed } & \text { Ord }\end{array}$ http://ramesesproject.org/media/RE_Quality_Standards_for_evaluators_and_peer_revi ewers.pdf

Guba, E. G., \& Lincoln, Y. S. (1994). Competing paradigms in qualitative research. Handbook of qualitative research. 163-194.

Gwinner, K., \& Ward, L. (2015). Storytelling, Safeguarding, Treatment, and Responsibility: attributes of recovery in psychiatric intensive care units. Journal of Psychiatric Intensive Care, 11(2), 105-118.

Hammersley, M., \& Atkinson, P. (2007). Ethnography: Principles in practice. Routledge.

Hansen, H. F. (2005). Choosing evaluation models: a discussion on evaluation design. Evaluation, 11(4), 447-462.

Helman, C.G. (2007). Culture health and illness. International Review of Psychiatry, 21(5), 489-489.

Henderson, J. (2005) Neo-liberalism, community care and Australian mental health policy. Health Sociology review, 14(3), 242-254.

Henderson, C., Noblett, J., Parke, H., Clement, S., Caffrey, A., Gale-Grant, O., \& Thornicroft, G. (2014). Mental health-related stigma in health care and mental health-care settings. The Lancet Psychiatry, 1(6), 467-482.

Hendryx, M., Green, C. A., \& Perrin, N. A. (2009). Social support, activities, and recovery from serious mental illness: STARS study findings. The journal of behavioral health services \& research, 36(3), 320-329.

Hermanowicz, J. C. (2002). The great interview: 25 strategies for studying people in bed. Qualitative Sociology, 25(4), 479-499.

Herzberg, F., Mausner, B., \& Snyderman, B. B. (1959). The Motivation to Work, John Wiley 
\& Sons. Inc., New York, 195.

Hilal, A. H., \& Alabri, S. S. (2013). Using NVivo for data analysis in qualitative research. International interdisciplinary journal of education, 2(2), 181-186.

Holt-Lunstad, J. (2018). Why social relationships are important for physical health: A systems approach to understanding and modifying risk and protection. Annual review of psychology, 69, 437-458.

Holt-Lunstad, J., Smith, T. B., Baker, M., Harris, T., \& Stephenson, D. (2015). Loneliness and social isolation as risk factors for mortality: a meta-analytic review. Perspectives on psychological science, 10(2), 227-237.

Hopkins, J. E., Loeb, S. J., \& Fick, D. M. (2009). Beyond satisfaction, what service users expect of inpatient mental health care: a literature review. Journal of Psychiatric and Mental Health Nursing, 16(10), 927-937.

Horwitz, A. V., \& Scheid, T. L. (Eds.). (1999). A handbook for the study of mental health: Social contexts, theories, and systems. Cambridge University Press.

Huber, C. G., Schneeberger, A. R., Kowalinski, E., Fröhlich, D., von Felten, S., Walter, M., \& Lang, U. E. (2016). Suicide risk and absconding in psychiatric hospitals with and without open door policies: a 15 year, observational study. The Lancet Psychiatry, 3(9), 842-849.

Hungerford, C., \& Richardson, F. (2013). Operationalising Recovery-oriented services: The challenges for carers. Advances in Mental Health, 12(1), 11-21.

Hungerford, C., \& Fox, C. (2014). Consumer's perceptions of Recovery-oriented mental health services: An Australian case-study analysis. Nursing \& health sciences, 16(2), 209215.

Jackson, D. (2009). The importance of optimism. Journal of Clinical Nursing, 18, 13771378.

Jacobson, N., \& Curtis, L. (2000). Recovery as policy in mental health services: Strategies emerging from the states. Psychiatric Rehabilitation Journal, 23(4), 333.

Jacobson, N., \& Greenley, D. (2001). What is recovery? A conceptual model and explication. Psychiatric services, 52(4), 482-485.

Jagosh, J. (2018). Introduction to Realist Evaluation and Synthesis, $19^{\text {th }}$ February 2018, London.

Jas, E., \& Wieling, M. (2018). Providers' competencies positively affect personal recovery of involuntarily admitted patients with severe mental illness: A prospective observational study. International Journal of Social Psychiatry, 64(2), 145-155.

Jenner, S. (2005). Social construction as a research tool-Building a new model based on grounded theory and heuristic research. 
Joosten, E. A., DeFuentes-Merillas, L., De Weert, G. H., Sensky, T., Van Der Staak, C. P. F., \& de Jong, C. A. (2008). Systematic review of the effects of shared decision-making on patient satisfaction, treatment adherence and health status. Psychotherapy and psychosomatics, 77(4), 219-226.

Jordan, K., Porcheret, M., \& Croft, P. (2004). Quality of morbidity coding in general practice computerized medical records: a systematic review. Family practice, 21(4), 396412.

Kaiser, K. (2009). Protecting respondent confidentiality in qualitative research. Qualitative health research, 19(11), 1632-1641.

Karasz, A., Garcia, N., \& Ferri, L. (2009). Conceptual models of depression in primary care patients: A comparative study. Journal of Cross-Cultural Psychology. 40. 1041-1059.

Katsakou, C., Marougka, S., Barnicot, K., Savill, M., White, H., Lockwood, K., \& Priebe, S. (2012). Recovery in borderline personality disorder (BPD): a qualitative study of service users' perspectives. PloS one, 7(5).

Keetharuth, A. D., Brazier, J., Connell, J., Carlton, J., Taylor Buck, E., \& Ricketts, T. (2017). Development and Validation of the Recovering Quality of Life (ReQoL) Outcome Measures. EEPRU Technical Research Report 050. Policy Research Unit in Economic Evaluation of Health and Care Interventions, Universities of Sheffield and York.

Kelly, M., \& Gamble, C. (2005). Exploring the concept of recovery in schizophrenia. Journal of Psychiatric and Mental Health Nursing, 12(2), 245-251.

Kelly, B. D. (2005). Structural violence and schizophrenia. Social science \& medicine, 61(3), 721-730.

Killaspy, H., King, M., Holloway, F., Craig, T. J., Cook, S., Mundy, T., \& Marston, L. (2017). The Rehabilitation Effectiveness for Activities for Life (REAL) study: a national programme of research into NHS inpatient mental health rehabilitation services across England.

King, A. J., \& Simmons, M. B. (2018). A systematic review of the attributes and outcomes of peer work and guidelines for reporting studies of peer interventions. Psychiatric services, 69(9), 961-977.

Knaak, S., Mantler, E., \& Szeto, A. (2017). Mental illness-related stigma in healthcare: Barriers to access and care and evidence-based solutions. In Healthcare management forum. 30, (2), 111-116. Sage CA: Los Angeles, CA: SAGE Publications.

Kornhaber, R., Walsh, K., Duff, J., \& Walker, K. (2016). Enhancing adult therapeutic interpersonal relationships in the acute health care setting: An integrative review. Journal of multidisciplinary healthcare, 9, 537.

Kuhn, T. (1970). The Structure of Scientific Revolutions (2nd ed.). Chicago: University of Chicago Press.

Lakeman, R. (2016). Paradoxes of personal responsibility in mental health care. Issues in mental health nursing, 37(12), 929-933. 
Lakoff, G. (1987). Women, fire, and dangerous things: What categories reveal about the mind. Chicago: University of Chicago Press.

Laugharne, R., Priebe, S., McCabe, R., Garland, N., \& Clifford, D. (2012). Trust, choice and power in mental health care: Experiences of patients with psychosis. International Journal of Social Psychiatry, 58(5), 496-504.

Leamy, M., Bird, V., Le Boutillier, C., Williams, J., \& Slade, M. (2011). Conceptual framework for personal recovery in mental health: systematic review and narrative synthesis. The British Journal of Psychiatry, 199(6), 445-452.

Le Boutillier, C., Slade, M., Lawrence, V., Bird, V. J., Chandler, R., Farkas, M., \& Shepherd, G. (2015). Competing priorities: staff perspectives on supporting recovery. Administration and Policy in Mental Health and Mental Health Services Research, 42(4), 429-438.

Lee, R. M. (1993). Doing research on sensitive topics. Sage. 4-5.

Légaré, F., Ratté, S., Gravel, K., \& Graham, I. D. (2008). Barriers and facilitators to implementing shared decision-making in clinical practice: update of a systematic review of health professionals' perceptions. Patient education and counselling, 73(3), 526-535.

Leung, J., \& Arthur, D. G. (2004). Clients and facilitators' experiences of participating in a Hong Kong self-help group for people recovering from mental illness. International journal of mental health nursing, 13(4), 232-241.

Liebenberg, L., Ungar, M., \& Ikeda, J. (2015). Neo-liberalism and responsibilisation in the discourse of social service workers. British Journal of Social Work, 45(3), 1006-1021.

Lieberman, J. A., Drake, R. E., Sederer, L. I., Belger, A., Keefe, R., Perkins, D., \& Stroup, S. (2008). Science and recovery in schizophrenia. Psychiatric Services, 59(5), 487-496.

Lofland, J., Snow, D., Anderson, L., \& Lofland, L. H. (2006). Analyzing Social Settings: A Guide to Qualitative Observation and Analysis. Belmont, CA: Wadsworth/Thomson Learning.

Long, A. F., \& Godfrey, M. (2004). An evaluation tool to assess the quality of qualitative research studies. International Journal of Social Research Methodology, 7(2), 181-196.

Lorant, V., Grard, A., \& Nicaise, P. (2016). Implementing a Nation-Wide Mental Health Care reform: an analysis of stakeholders' priorities. Community mental health journal, 52(3), 343-352.

Lynch, M. (2000). Against reflexivity as an academic virtue and source of privileged knowledge. Theory, Culture \& Society, 17(3), 26-54.

Mahon, J. F. (2002). Corporate reputation: Research agenda using strategy and stakeholder literature. Business \& Society, 41(4), 415-445.

Mancini, M. A., Hardiman, E. R., \& Lawson, H. A. (2005). Making sense of it all: consumer providers' theories about factors facilitating and impeding recovery from psychiatric disabilities. Psychiatric rehabilitation journal, 29(1), 48. 
Manzano, A. (2016). The craft of interviewing in realist evaluation. Evaluation, 22(3), 342360.

Marchal, B., van Belle, S., van Olmen, J., Hoeree, T., \& Kegels G. (2012). Is realist evaluation keeping its promise? A review of published empirical studies in the field of health systems research. Evaluation. 18. (2) 192-212.

Mattingly, C. (2010). The Paradox of Hope: Journeys through a Clinical Borderland, Berkeley, University of California Press.

Maxwell, J. A. (2008). The value of a realist understanding of causality for qualitative research. In Denzin, N. (Ed.). Qualitative research and the politics of evidence. 163-181.

May, T., \& Perry, B. (2014). Reflexivity and the practice of qualitative research. Los Angeles: SAGE.

McAndrew, S., Chambers, M., Nolan, F., Thomas, B., Watts, P. (2014). Measuring the evidence: Reviewing the literature of the measurement of therapeutic engagement. International Journal of Mental Health Nursing, 23, 212-220.

McCoy, M. S., Jongsma, K. R., Friesen, P., Dunn, M., Neuhaus, C. P., Rand, L., \& Sheehan, M. (2018). National Standards for Public Involvement in Research: missing the forest for the trees. Journal of Medical Ethics, 44(12), 801-804.

McKeown, M., Wright, K. \& Mercer, D. (2017). Care planning: a neoliberal three card trick. Journal of Psychiatric and Mental Health Nursing, 24(6), 451-460

McKenna, B., Furness, T., Dhital, D., Ennis, G., Houghton, J., Lupson, C., \& Toomey, N. (2014). Recovery-oriented care in acute inpatient mental health settings: An exploratory study. Issues in Mental Health Nursing, 35(7), 526-532.

McPherson, P., Krotofil, J., \& Killaspy, H. (2018). Mental health supported accommodation services: a systematic review of mental health and psychosocial outcomes. BMC psychiatry, 18(1), 1-15.

McWade, B. (2016). Recovery-as-policy as a form of neoliberal state making. Intersectionalities: A Global Journal of Social Work Analysis, Research, Polity, and Practice, 5(3), 62-81.

Mead, S., Hilton, D., \& Curtis, L. (2001). Peer support: A theoretical perspective. Psychiatric rehabilitation journal, 25(2), 134.

Mental Health Commission of Canada. (2012) Changing directions, changing lives: the mental health strategy for Canada. Calgary: Mental Health Commission of Canada.

Metz, M. J., Veerbeek, M. A., van der Feltz-Cornelis, C. M., de Beurs, E., \& Beekman, A. T. (2018). Decisional conflict in mental health care: a cross-sectional study. Social psychiatry and psychiatric epidemiology, 53(2), 161-169.

Milbourn, B. T., McNamara, B. A., \& Buchanan, A. J. (2014). Do the everyday experiences of people with severe mental illness who are "hard to engage" reflect a journey of personal recovery? Journal of Mental Health, 35(5), 241- 245. 
Mol, A., \& Law, J. (2004). Embodied action, enacted bodies: The example of hypoglycaemia. Body \& society, 10(2-3), 43-62.

Molder, B. (2010) Mind Ascribed: an elaboration and defence of interpretivism. Philadelphia USA: John Benjamins.

Morera, T., Pratt, D., \& Bucci, S. (2017). Staff views about psychosocial aspects of recovery in psychosis: A systematic review. Psychology and Psychotherapy: Theory, Research and Practice, 90(1), 1-24.

Morgen, S. (2001) The Agency of Welfare Workers: Negotiating Devolution, Privitization, and the Meaning of Self-Sufficiency. American Anthropologist, 103(3), 747-761.

Morris, J. (1997). Care of empowerment? A disability rights perspective. Social Policy \& Administration, 31(1), 54-60.

Morrow, M. (2013). Recovery: Progressive Paradigm or Neoliberal Smokescreen? In B.A. LeFrancois, R. Menzies \& G. Reaume Mad Matters: A Critical Reader in Canadian Mad Studies. Toronto: Canadian Scholar's Press.

Muir-Cochrane, E., Oster, C., Grotto, J., Gerace, A., \& Jones, J. (2013). The inpatient psychiatric unit as both a safe and unsafe place: Implications for absconding. International Journal of Mental Health Nursing, 22(4), 304-312.

Nadler, D. A., \& Tushman, M. L. (1980). A model for diagnosing organizational behavior. Organizational Dynamics, 9(2), 35-51.

National Collaborating Centre for Methods and Tools. (2011) Critical appraisal tools to make sense of evidence. Hamilton, ON: McMaster University. Retrieved 20 ${ }^{\text {th }}$ March 2020, from http://www.nccmt.ca/ resources/search/87.

National Collaborative Commissioning Unit (2020). NHS Wales Quality Assurance Improvement Service 8th Annual Position Statement 2019-2020. Available at: http://www.wales.nhs.uk/sites3/page.cfm?orgid=480\&pid=99597 (accessed on $13^{\text {th }}$ August 2020).

National Institute for Health Research. (2016) NIHR annual report 2015/16: National Institute for Health Research. Available at: https://www.nihr.ac.uk/documents/aboutus/our-contribution-to-research/research-performance/NIHR-ANNUAL-REPORT-201617.pdf. (accessed on $9^{\text {th }}$ July 2020 ).

New Freedom Commission on Mental Health. (2003) Achieving the promise: transforming mental health care in America. Final report. Rockville: U.S. Department of Health and Human Services.

Newing, H. (2010). Conducting research in conservation: social science methods and practice. Routledge.

NICE. (2011). Service user experience in adult mental health services. London: National Institute for Mental Health. 
Noordsy, D., Torrey, W., Mueser, K., Mead, S., O'Keefe, C., \& Fox, L. (2002). Recovery from severe mental illness: an intrapersonal and functional outcome definition. International Review of Psychiatry, 14(4), 318-326.

Novick, G. (2008). Is there a bias against telephone interviews in qualitative research? Research in Nurshing \& Health, 31, 391-398.

Oades, L., Deane, F., Crowe, T., Gordon Lambert, W., Kavanagh, D., \& Lloyd, C. (2005). Collaborative recovery: An integrative model for working with individuals who experience chronic and recurring mental illness. Australasian Psychiatry, 13(3), 279-284.

O'Cathain, A., Murphy, E., \& Nicholl, J. (2010). Three techniques for integrating data in mixed methods studies. BMJ, 341.

Oborn, E., Barrett, M., Gibson, S., \& Gillard, S. (2019). Knowledge and expertise in care practices: the role of the peer worker in mental health teams. Sociology of health \& illness, 41(7), 1305-1322.

Ogrinc, G., \& Batalden, P. (2009). Realist evaluation as a framework for the assessment of teaching about the improvement of care. Journal of Nursing Education, 48(12), 661-667.

Oliver, M, (2004). The social model of action: if I had a hammer. In Barnes, C., \& Mercer, G. (Eds.). Implementing the Social Model of Disability: Theory and research. Leeds: Disability Press.

Onken, S. J., Dumont, J. M., Ridgway, P., Dornan, D. H., \& Ralph, R. O. (2002). Mental health recovery: What helps and what hinders? A national research project for the development of recovery facilitating system performance indicators. Prepared for National Technical Assistance Centre for State Mental Health Planning, National Association of State Mental Health Program Directors.

Onken, S. J., Craig, C. M., Ridgway, P., Ralph, R. O., \& Cook, J. A. (2007). An analysis of the definitions and elements of recovery: a review of the literature. Psychiatric rehabilitation journal, 31(1), 9.

Opdenakker, R. (2006). Advantages and Disadvantages of Four Interview Techniques in Qualitative Research. Forum: Qualitative Social Research, 7(4).

Osborn, L. A., \& Stein, C. H. (2019). Recovery-oriented services in an inpatient setting: The role of consumers' views of therapeutic alliance and practitioner directiveness on recovery and well-being. American Journal of Orthopsychiatry, 89(1), 115.

Owen-Smith, A., Bennewith, O., Donovan, J., Evans, J., Hawton, K., Kapur, N., \& Gunnell, D. (2014). When You're in the Hospital, You're in a Sort of Bubble. Understanding the high risk of self-harm and suicide following psychiatric discharge: a qualitative study. Crisis.

Palumbo, C., Volpe, U., Matanov, A., Priebe, S., \& Giacco, D. (2015). Social networks of patients with psychosis: a systematic review. BMC research notes, 8(1), 560.

Patton (2003). Qualitative evaluation checklist. Evaluation Checklists Project.

Parker, S., Dark, F., Newman, E., Korman, N., Rasmussen, Z., \& Meurk, C. (2017). Reality of working in a community-based, recovery-oriented mental health rehabilitation unit: $\mathrm{A}$ 
pragmatic grounded theory analysis. International Journal of Mental Health Nursing, 26(4), 355-365.

Pawson, R. (1996). Theorizing the interview. The British Journal of Sociology. 42. 20.

Pawson, R., Greenhalgh, T., Harvey, G., \& Walshe, K. (2004). Realist synthesis: an introduction. Manchester: ESRC Research Methods Programme, University of Manchester.

Pawson, R. (2006). Evidence-based policy: a realist perspective. London, SAGE.

Pawson, R. (2013). The Science of Evaluation: A Realist Manifesto. London, SAGE.

Pawson, R. \& Manzano-Santaella, A. (2012). A realist diagnostic workshop. Evaluation. 18. (2). 176-191.

Pawson, R. \& Tilley, N. (1997). Realistic Evaluation, London, Sage.

Peacock, M., Bissell, P., \& Owen, J. (2014). Dependency denied: health inequalities in the neo-liberal era. Social Science \& Medicine, 118, 173-180.

Pearlman, V., \& Holzhausen, E. (2002). Adding Value: Carers as drivers of social change. Carers UK.

Peplau L.A., \& PerIman, D. (1982). Perspectives on loneliness. In: Peplau LA, Perlman D (eds) Loneliness: a sourcebook of current theory, research, and therapy. Wiley, New York, $1-18$.

Perkins, R., \& Slade, M. (2012). Recovery in England: transforming statutory services?. Int Rev Psychiatry, 24, 29-39.

Pharoah, F., Mari, J. J., Rathbone, J., \& Wong, W. (2010). Family intervention for schizophrenia. Cochrane database of systematic reviews, (12).

Piat, M., Sofouli, E., Sabetti, J., Lambrou, A., Chodos, H., Briand, C., \& Curran, J. (2017). Protocol for a mixed studies systematic review on the implementation of the recovery approach in adult mental health services. BMJ Open, 7(8).

Pilgrim, D. (2008). Recovery and current mental health policy. Chronic illness, 4(4), 295304.

Pilgrim, D. (2009) Key Concepts in Mental Health. Second Edition. London. Sage.

Pincus, H. A., Spaeth-Rublee, B., Sara, G., Goldner, E. M., Prince, P. N., Ramanuj, P., \& van Weeghel, J. (2016). A review of mental health recovery programs in selected industrialized countries. International journal of mental health systems, 10(1), 73.

Prochaska, J. O., \& DiClemente, C. C. (1982). Transtheoretical therapy: toward a more integrative model of change. Psychotherapy: theory, research \& practice, 19(3), 276.

Pritchard, A. (2018). Advice for managing multiple funders, their expectations and agendas. PSYPag The Quarterly. 109. 40-42. 
Ramanuj, P. P., Carvalho, C. F., Harland, R., Garety, P. A., Craig, T. K. \& Byrne, N. (2015). Acute mental health service use by patients with severe mental illness after discharge to primary care in South London. Journal of Mental Health, 24(4), 208-213.

Ramon, S., Healy, B., \& Renouf, N. (2007). Recovery from mental illness as an emergent concept and practice in Australia and the UK. International Journal of Social Psychiatry, 53(2), 108-122.

Raven, M. C., Doran, K. M., Kostrowski, S., Gillespie, C. C., \& Elbel, B. D. (2011). An intervention to improve care and reduce costs for high-risk patients with frequent hospital admissions: a pilot study. BMC health services research, 11(1), 270.

Reason, P., \& Bradbury, H. (Eds.). (2001). Handbook of action research: Participative inquiry and practice. Sage.

Recovery in the Bin. (2019). RITB - Key Principles. Available: https://recoveryinthebin.org/ritbkeyprinciples/ (accessed 30 th March 2020).

Reis, H. T., \& Shaver, P. (1988). Intimacy as an interpersonal process. Handbook of personal relationships.

Repper, J. \& Perkins, R. (2003) Social Inclusion and Recovery. A Model for Mental Health Practice. London: Balliere Tindall.

Riege, A.M. (2003), Validity and reliability tests in case study research: a literature review with 'hands-on' applications for each research phase, Qualitative Market Research: An International Journal. 6. (2) 75-86.

Riessman, C. K. (1993). Narrative analysis, 30. Sage.

Ritchie, J. \& Spencer, L., 1994. Qualitative data analysis for applied policy research. In B. Bryman \& R. Burgess, Analysing qualitative data. 173-194.

Roberts, L. W., \& Kim, J. P. (2014). Giving voice to study volunteers: Comparing views of mentally ill, physically ill, and healthy protocol participants on ethical aspects of clinical research. Journal of psychiatric research, 56, 90-97.

Rogers, P. J. (2008). Using programme theory to evaluate complicated and complex aspects of interventions. Evaluation, 14(1), 29-48.

Rose, N. (1996). The death of the social? Re-figuring the territory of government. International Journal of Human Resource Management, 25(3), 327-356.

Rose, D. (2014). The mainstreaming of recovery. Journal of Mental Health, 23 (5), 217218.

Russinova, Z., Rogers, E. S., Ellison, M. L., \& Lyass, A. (2011). Recovery-promoting professional competencies: Perspectives of mental health consumers, consumerproviders and providers. Psychiatric Rehabilitation Journal, 34(3), 177. 
Rycroft-Malone, J., Fontenla, M., Bick, D., \& Seers, K. (2010). A realistic evaluation: the case of protocol-based care. Implementation science, 5(1), 38.

Salyers, M. P., \& Tsemberis, S. (2007). ACT and recovery: Integrating evidence-based practice and recovery orientation on assertive community treatment teams. Community Mental Health Journal, 43(6), 619-641.

Sayer, A. (2000) Realism and Social Science. London: Sage.

Schrank, B., Hayward, M., Stanghellini, G., \& Davidson, L. (2011). Hope in psychiatry. Advances in Psychiatric Treatment, 17(3), 227-235.

Schultz, E. M., \& McDonald, K. M. (2014). What is care coordination? International Journal of Care Coordination, 17(12), 5-25.

Schwartz, B., \& Ward, A. (2004). Doing better but feeling worse: The paradox of choice. Positive psychology in practice, 86-104.

Schwartz, R., Estein, O., Komaroff, J., Lamb, J., Myers, M., Stewart, J., \& Park, M. (2013). Mental health consumers and providers dialogue in an institutional setting: $A$ participatory approach to promoting recovery-oriented care. Psychiatric Rehabilitation Journal, 36(2), 113.

Scott, M. B., \& Lyman, S. M. (1968). Accounts. American sociological review, 46-62.

Searle, J. R., \& Willis, S. (1995). The construction of social reality. Simon and Schuster.

Shahar, G., Trower, P., Iqbal, Z., Birchwood, M., Davidson, L., \& Chadwick, P. (2004). The person in recovery from acute and severe psychosis: the role of dependency, selfcriticism, and efficacy. American Journal of Orthopsychiatry, 74(4), 480-488.

Sharac, J., McCrone, P., Clement, S., \& Thornicroft, G. (2010). The economic impact of mental health stigma and discrimination: A systematic review. Epidemiology and Psychiatric Sciences, 19(3), 223-232.

Sharac, J., McCrone, P., Sabes-Figuera, R., Csipke, E., Wood, A., \& Wykes, T. (2010). Nurse and patient activities and interaction on psychiatric inpatients wards: a literature review. International journal of nursing studies, 47(7), 909-917.

Shearn, K., Allmark, P., Piercy, H., \& Hirst, J. (2017). Building realist program theory for large complex and messy interventions. International Journal of Qualitative Methods, 16(1).

Shepherd, G., Boardman, J., \& Slade, M. (2008). Making recovery a reality (pp. 1-3). London: Sainsbury Centre for mental health.

Shepherd, A., Shorthouse, O., \& Gask, L. (2014). Consultant psychiatrists' experiences of and attitudes towards shared decision making in antipsychotic prescribing, a qualitative study. BMC psychiatry, 14(1), 127.

Sieber, J. E., \& Stanley, B. (1988). Ethical and professional dimensions of socially sensitive research. American psychologist, 43(1), 49. 
Silverstein, S. M., \& Bellack, A. S. (2008). A scientific agenda for the concept of recovery as it applies to schizophrenia. Clinical psychology review, 28(7), 1108-1124.

Simpson, A., \& Penney, S. (2011). The recovery paradigm in forensic mental health services. Criminal Behaviour and Mental Health, 21(5), 299-306.

Simpson, A., Oster, C., \& Muir-Cochrane, E. (2018). Liminality in the occupational identity of mental health peer support workers: A qualitative study. International journal of mental health nursing, 27(2), 662-671.

Slade, M. (2009). Personal Recovery and Mental illness: A guide for Mental Health Practitioners. Cambridge, UK, Cambridge University Press.

Slade, M., Adams, N., \& O'Hagan, M. (2012). Recovery: past progress and future challenges. Int. Rev. Psychiatry. 24, 1-4.

Slade, M., Williams, J., Bird, V., Leamy, M. and Le Boutillier, C. (2012) 'Recovery grows up'. Journal of Mental Health, 21(2), 99-104.

Slade, M., Amering, M., Farkas, M., Hamilton, B., O'Hagan, M., Panther, G., \& Whitley, R. (2014). Uses and abuses of recovery: implementing recovery-oriented practices in mental health systems. World Psychiatry, 13(1), 12-20.

Slade, M., \& Longden, E. (2015). Empirical evidence about recovery and mental health. BMC psychiatry, 15(1), 285.

Slade, M. (2017). Implementing shared decision making in routine mental health care. World psychiatry, 16(2), 146-153.

Soss, J., Fording, R. C., Schram, S. F., \& Schram, S. (2011). Disciplining the poor: Neoliberal paternalism and the persistent power of race. University of Chicago Press.

Soss, J., Fording, R. C., \& Schram, S. F. (2009). Governing the poor: the rise of the neoliberal paternalist state. In Annual Meeting of the American Political Science Association, Toronto.

Stewart, J. L. (2019). The ghettoization of persons with severe mental illnesses. Mental Health and Social Inclusion.

Stickley, T., \& Felton, A. (2006). Promoting recovery through therapeutic risk taking. Mental Health Practice, 9(8).

Stickley, T., Wright, N., Slade, M. (2018). The art of recovery: outcomes from participatory arts activities for people using mental health services, Journal of Mental Health, 27:4, 367373.

Storey, K., Shute, T., \& Thompson, A. (2008). Building a culture of recovery: a comprehensive recovery education strategy. Journal of Ethics in Mental Health, 3, 1- 4.

Stylianos, S., \& Kehyayan, V. (2012). Advocacy: Critical component in a comprehensive mental health system. American Journal of Orthopsychiatry, 82(1), 115.

Srivastava, A., \& Thomson, S. B. (2009). Framework analysis: a qualitative methodology for applied policy research. 
Sullivan, M. (1994) Modern Social Policy. Hertfordshire: Harvester Wheatsheaf.

Szasz, T. (2009). Coercion as cure: A critical history of psychiatry. New Brunswick, N.J. London: Transaction Publishers.

Taylor, A. (2002). I'll call you back on my mobile: A critique of the telephone interview with adolescent boys. Westminster Studies in Education, 25(1), 19-34.

Taylor, G. W., \& Ussher, J. M. (2001). Making sense of S\&M: A discourse analytic account. Sexualities, 4(3), 293-314.

Teghtsoonian, K. (2009). Depression and mental health in neoliberal times: A critical analysis of policy and discourse. Social Science \& Medicine, 69(1), 28-35.

Tennant, R., Hiller, L., Fishwick, R., Platt, S., Joseph, S., Weich, S., \& Stewart-Brown, S. (2007). The Warwick-Edinburgh mental well-being scale (WEMWBS): development and UK validation. Health and Quality of life Outcomes, 5(1), 63.

Terry, J., \& Coffey, M. (2019). Too Busy to Talk: Examining service user involvement in nursing work. Issues in mental health nursing, 40(11), 957-965.

Tew, J., Ramon, S., Slade, M., Bird, V., Melton, J., \& Le Boutillier, C. (2012). Social factors and recovery from mental health difficulties: a review of the evidence. The British Journal of Social Work, 42(3), 443-460.

Thompson, J., Bissell, P., Cooper, C. L., Armitage, C. J., \& Barber, R. (2014). Exploring the impact of patient and public involvement in a cancer research setting. Qualitative health research, 24(1), 46-54.

Thornicroft, G., Rose, D., \& Kassam, A. (2007). Discrimination in health care against people with mental illness. International review of psychiatry, 19(2), 113-122.

Thornton, T., Crepaz-Keay, D., Birch, S., \& Verhaegh, J. (2017). Generating nursing knowledge. Psychiatric and Mental Health Nursing: The craft of caring.

Tilley, N. (1998). Evaluating the effectiveness of CCTV schemes. Surveillance, closed circuit television and social control, 139-153.

Tilley, N. (2000). The evaluation jungle. In: Ballintyne S, Pease K and McLaren V (eds) Secure Foundations: Key Issues in Crime Prevention, Crime Reduction and Community Safety. London: Institute for Public Policy Research.

Tolson, D., McIntosh, J., Loftus, L., \& Cormie, P. (2007). Developing a managed clinical network in palliative care: a realistic evaluation. International journal of nursing studies, 44(2), 183-195.

Tsai, J., \& Salyers, M. P. (2010). Recovery orientation in hospital and community settings. The journal of behavioural health services \& research, 37(3), 385-399.

Tudge, J. R., Payir, A., Merçon-Vargas, E., Cao, H., Liang, Y., Li, J., \& O'Brien, L. (2016). Still misused after all these years? A reevaluation of the uses of Bronfenbrenner's bioecological theory of human development. Journal of Family Theory \& Review, 8(4), 427-445. 
Turner, V. (1969). From Ritual to Theatre: The Human Seriousness of Play. Chicago: Aldione.

Turner, J. C., Hogg, M. A., Oakes, P. J., Reicher, S. D., \& Wetherell, M. S. (1987). Rediscovering the social group: A self-categorization theory. Basil Blackwell.

Ungar, M., Ghazinour, M., \& Richter, J. (2013). Annual research review: What is resilience within the social ecology of human development?. Journal of child psychology and psychiatry, 54(4), 348-366.

Vygotsky, L. (1978). Interaction between learning and development. Readings on the development of children, 23(3), 34-41.

Waldemar, A. K., Arnfred, S. M., Petersen, L., \& Korsbek, L. (2015). Recovery-oriented practice in mental health inpatient settings: A literature review. Psychiatric Services, 67(6), 596-602.

Waldemar, A. K., Esbensen, B. A., Korsbek, L., Petersen, L., \& Arnfred, S. (2018a). Recoveryoriented practice: Participant observations of the interactions between patients and health professionals in mental health inpatient settings. International journal of mental health nursing, 28(1), 318-329.

Waldemar, A. K., Esbensen, B. A., Korsbek, L., Petersen, L., \& Arnfred, S. (2018b). Recovery orientation in mental health inpatient settings: Inpatient experiences?. International journal of mental health nursing, 27(3), 1177-1187.

Wanat, C. L. (2008). Getting past the gatekeepers: Differences between access and cooperation in public school research. Field methods, 20(2), 191-208.

Watkins, M., \& Shulman, H. (2010). Toward psychologies of liberation. Palgrave.

Watts, M. (2012). Recovery from 'Mental Illness' as a Re- enchantment with Life: a narrative study, unpublished PhD, University of Dublin, Trinity College.

Weaver, N., Coffey, M., \& Hewitt, J. (2017). Concepts, models and measurement of continuity of care in mental health services: a systematic appraisal of the literature. Journal of Psychiatric and Mental Health Nursing, 24(6), 431-450.

Welsh Government. (2011). Implementing the Mental Health (Wales) Measure 2010: Guidance for local health boards and local authorities. Cardiff: Welsh Government.

Welsh Government. (2015). The duty to review final report: post-legislative assessment of the Mental Health (Wales) Measure 2010. Cardiff: Welsh Government.

Westhorp, G., \& Manzano., A. (2017). Realist Evaluation Interviewing - A 'Starter Set' of Questions. The RAMESES II Project. Viewed $3^{\text {rd }}$ October 2019 https://www.ramesesproject.org/media/RAMESES_II_Realist_interviewing_starter_ques tions.pdf

Williams, L., Burton, C., \& Rycroft Malone, J. (2013) What works: a realist evaluation case study of intermediaries in infection control practice. Journal of Advanced Nursing. 69.(4). 915-926. 
Whitley, R., Gingerich, S., Lutz, W. J., \& Mueser, K. T. (2009). Implementing the illness management and recovery program in community mental health settings: facilitators and barriers. Psychiatric Services, 60(2), 202-209.

Whyte, W. F. (1993). Street corner society: The social structure of an Italian slum. Chicago, IL: University of Chicago Press.

Wong, G., Greenhalgh, T., Westhorp, G., \& Pawson, R. (2012). Realist methods in medical education research: what are they and what can they contribute?. Medical education, 46(1), 89-96.

Wong, G., Westhorp, G., Manzano, A., Greenhalgh, J., Jagosh, J., \& Greenhalgh, T. (2016). RAMESES II reporting standards for realist evaluations. BMC medicine, 14(1), 96.

World Health Organisation. (2008). Integrating mental health into primary care: a global perspective. Helsinki: World Health Organization.

Young, S. L., \& Ensing, D. S. (1999). Exploring recovery from the perspective of people with psychiatric disabilities. Psychiatric rehabilitation journal, 22(3), 219. 
APPENDICES 


\section{Appendix A - Programme Architect and Staff Participant Information Sheet}

\section{Participant Information Sheet - A Realist Evaluation of Woodlands}

You are being invited to take part in some research. Before you decide whether or not to participate, it is important for you to understand why the research is being conducted and what it will involve. Please read the following information carefully.

\section{What is the purpose of the research?}

The aim of this project is to evaluate the Philosophy of Care at Woodlands. The aim is to understand what is and is not currently working at Woodlands and to understand why and how it is or is not working. Your participation in this study will take approximately 1 hour in the form of an interview.

\section{Why have I been chosen?}

You have been chosen as you are a staff member at Woodlands and will be able to provide valuable information to enable the researcher to evaluate the services available at Woodlands. Clients and their carers will also be approached to see if they would like to participate in the research project.

\section{Who is carrying out the research?}

The data are being collected by Amy Pritchard, who is part of the Psychology Department within College of Human and Health Sciences. The research has been approved by the College of Human and Health Sciences Research Ethics Committee.

\section{What happens if I agree to take part?}

You will be asked to participate in a one-hour interview about the whole person approach to care / philosophy of care at Woodlands. You will be asked if you still consent to participate in the research and will be required to fill out a paper form demonstrating you understand the research process. All the interviews will be audio recorded, but the audio tapes will be destroyed once they have been typed up by Amy. The data will be stored securely on a password protected computer and paper forms will be in a locked cupboard. All data will be pseudonymised prior dissemination.

\section{Are there any risks associated with taking part?}

The research has been approved by the College of Human and Health Sciences Research Ethics Committee. There are no significant risks associated with participation.

\section{Data Protection and Confidentiality}

Your data will be processed in accordance with the Data Protection Act 2018 and the General Data Protection Regulation 2016 (GDPR). All information collected about you will be kept strictly confidential. Your data will only be viewed by the researcher/research team.

All electronic data will be stored on a password-protected computer file at Swansea University. All paper records will be stored in a locked filing cabinet at Swansea University. Your consent information will be kept separately from your responses to minimise risk in the event of a data breach. 
Please note that the data we will collect for our study will be made anonymous, this will take place immediately after the interview as a pseudonym will be used, thus it will not be possible to identify and remove your data at a later date, should you decide to withdraw from the study. Therefore, if at the end of this research you decide to have your data withdrawn, please let me know before the $31^{\text {st }}$ December 2019.

\section{Will my taking part in this project be kept confidential?}

All information which is collected from you during the research project will be kept strictly confidential, and only the key researcher will have access to the data as it will be kept on a password-protected computer. This information refers to the consent forms you complete, the audio recordings of the interview and the transcript of your interview. Although if any safeguarding risks are identified then confidentiality will need to be breached, this includes if there is an identified risk to others or self.

On occasions, the key researcher may need to share information with her supervisory team, however, your identity will remain anonymous. All information from the research project will be destroyed after 7 years. Individual comments that you make during the interview will not be disclosed to the staff at Woodlands. Some anonymised direct quotations from interviews may be reported within my PhD thesis and other publications.

\section{What will happen to the information I provide?}

An analysis of the information will form part of our report at the end of the study and may be presented to interested parties and published in scientific journals and related media. All information presented in any reports or publications will be anonymous and unidentifiable.

Is participation voluntary and what if I wish to later withdraw?

Your participation is entirely voluntary - you do not have to participate if you do not want to. If you decide to participate, but later wish to withdraw from the study, then you are free to withdraw at any time, without giving a reason and without penalty. You have until the $31^{\text {st }}$ December 2019 to do this.

\section{Data Protection Privacy Notice}

The data controller for this project will be Swansea University. The University Data Protection Officer provides oversight of university activities involving the processing of personal data and can be contacted at the Vice Chancellors Office.

Your personal data will be processed for the purposes outlined in this information sheet.

Standard ethical procedures will involve you providing your consent to participate in this study by completing the consent form that has been provided to you.

The legal basis that we will rely on to process your personal data will be processing is necessary for the performance of a task carried out in the public interest. This public interest justification is approved by the College of Human and Health Sciences Research Ethics Committee, Swansea University.

The legal basis that we will rely on to process special categories of data will be processing is necessary for archiving purposes in the public interest, scientific or historical research purposes or statistical purposes. 


\section{How long will your information be held?}

We will hold any personal data and special categories of data for 7 years. This length of time is justified as the PhD length is 3 years and this will allow for the publication and dissemination of findings.

\section{What are your rights?}

You have a right to access your personal information, to object to the processing of your personal information, to rectify, to erase, to restrict and to port your personal information. Please visit the University Data Protection webpages for further information in relation to your rights.

Any requests or objections should be made in writing to the University Data Protection Officer:-

University Compliance Officer (FOI/DP) Vice-Chancellor's Office

Swansea University

Singleton Park

Swansea

SA2 8PP

Email: dataprotection@swansea.ac.uk

\section{How to make a complaint}

If you are unhappy with the way in which your personal data has been processed you may in the first instance contact the University Data Protection Officer using the contact details above.

If you remain dissatisfied then you have the right to apply directly to the Information Commissioner for a decision. The Information Commissioner can be contacted at: -

Information Commissioner's Office,

Wycliffe House,

Water Lane,

Wilmslow,

Cheshire,

SK9 5AF

www.ico.org.uk

\section{What if I have other questions?}

If you have further questions about this study, please do not hesitate to contact us:

Amy Pritchard, Department of Psychology, Swansea University, 967181@swansea.ac.uk

Prof Jaynie Rance, Department of Psychology, Swansea University, j.y.rance@swansea.ac.uk 
Participant Consent Form - A Realist Evaluation of Woodlands

Researcher - Amy Pritchard

Supervisor - Jaynie Rance -

\begin{tabular}{|c|c|c|}
\hline & & $\begin{array}{l}\text { Participant } \\
\text { initial }\end{array}$ \\
\hline 1. & $\begin{array}{l}\text { I (the participant) confirm that I have read and understand the } \\
\text { information sheet for the above study which is attached to this } \\
\text { form. }\end{array}$ & \\
\hline 2. & $\begin{array}{l}\text { I understand that my participation is voluntary and that I am } \\
\text { free to withdraw at any time, without giving any reasons. }\end{array}$ & \\
\hline 3. & $\begin{array}{l}\text { I understand what my role will be in this research, and all my } \\
\text { questions have been answered to my satisfaction. }\end{array}$ & \\
\hline 4. & $\begin{array}{l}\text { I understand that I am free to ask any questions at any time } \\
\text { before and during the study. }\end{array}$ & \\
\hline 5. & $\begin{array}{l}\text { I have been informed that the information I provide will be } \\
\text { safeguarded. }\end{array}$ & \\
\hline 6. & $\begin{array}{l}\text { I am happy for the information I provide to be pseudonymised } \\
\text { in academic papers and other formal research outputs. }\end{array}$ & \\
\hline 7. & I am willing for my information to be audio recorded. & \\
\hline 8. & $\begin{array}{l}\text { I have been provided with a copy of the Participant } \\
\text { Information Sheet. }\end{array}$ & \\
\hline 9. & $\begin{array}{l}\text { I agree to the researchers processing my personal data in } \\
\text { accordance with the aims of the study described in the } \\
\text { Participant Information Sheet. }\end{array}$ & \\
\hline 10. & $\begin{array}{l}\text { I understand that my data will be securely stored for } 7 \text { years } \\
\text { and will then be destroyed. }\end{array}$ & \\
\hline
\end{tabular}

Thank you for your participation in this study. Your help is very much appreciated.

Print name of participant

Print name of researcher
Signature

Date

This study is being conducted by Swansea University, College of Human and Health Science.

When complete: Original copy for participant, one copy to be retained by researcher. 
Appendix C - Programme Architect and Staff Debrief Sheet

\section{Staff and Programme Architect Debrief Sheet}

Thank you for taking part in the interview today. Your time and effort throughout this research project has been hugely appreciated.

If you have any queries about this study, please do not hesitate to contact Amy using the email address provided below. As all data collection is anonymous if you would like your data to be removed from the study you will need to provide Amy with your study identifier which is the pseudonym (fake name) you chose for the interview.

Amy Pritchard - Email Address

\section{Useful Numbers and Resources}

If you feel as though you would like extra support after taking part in this research, please contact:

* Contact Jaynie Rance (Supervisor of Amy Pritchard) 
Hello!

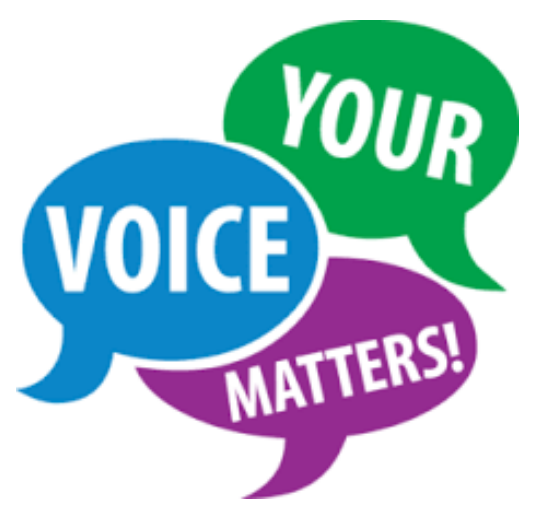

I would like to introduce myself and invite you to a briefing meeting at Woodlands on [insert date]. The meeting will be an opportunity for you to meet me and learn about the research I am doing for Woodlands.

I am Amy and I am a PhD student at Swansea University. Woodlands has asked me to independently evaluate the service, so they want to look at what is and is not working and the impact of the work they are currently doing. For me to do this I would like to listen to as many opinions as possible and would like to invite you to attend a briefing meeting so, you can consider whether you would like to share your views with me. I am interested in the views of clients, client's carers and staff, so others will receive a similar letter telling them about the research.

The briefing meeting is voluntary, so you do not have to attend, but if you would like to find out more information then please come along for a chat, drink and some biscuits. Attending the briefing meeting does not mean you are agreeing to participate in the research, it is just a chance to find out more and to give you more information, so you can make a choice as to whether you would like to participate. Participating in the research is also voluntary so if this is something you do not wish to be a part of then that is fine.

Thank you for taking the time to read this letter. I look forward to potentially seeing you on [insert date].

Best wishes,

Amy 


\section{Appendix E - Service-user Participant Information Sheet}

\section{Participant Information Sheet - A Realist Evaluation of Woodlands}

I would like to invite you to take part in a student research project. Before you decide you need to understand why the research is being done and what it will involve for you as a potential participant. Please take your time to read the following information and decide if you would like to participate in this research project. If you have any questions then please ask Amy, the researcher.

\section{Why is this research being done?}

- The aim of this project is to evaluate Woodlands approach to supporting you in your recovery.

- The aim is to understand what is and is not currently working at Woodlands and to understand why and how it is or is not working.

\section{Why have I been chosen?}

You have been chosen as you are a current client at Woodlands and will be able to provide valuable information to enable the researcher to evaluate Woodlands.

\section{Do I have to take part?}

This research is completely voluntary, and your care will not be affected if you decide not to take part. You will be provided with all the information about the project first and given some time to consider whether you would like to participate.

\section{What do I have to do if I agree to be part of the research project?}

- You will be invited to take part in up to three separate interviews with Amy, which will last up to 30 minutes each. There will be a gap between each interview of up to a couple of months. All the interviews will be audio recorded, but the audio tapes will be destroyed once they have been typed up by Amy.

- If you give your consent, the information from the questionnaires that you already complete at Woodlands will also be used for the evaluation. You will not need to do anything differently or be expected to fill in any more questionnaires.

\section{Nominating a Carer}

You will also be asked if you would like to nominate a carer to be contacted to participate in the research. It is not compulsory to nominate a carer and it will not affect your participation if you decide you do not wish to nominate anyone. When you are asked if you consent to the research, you will also be given a separate consent, where you will be asked to provide the name and contact information of the nominated individual and sign the form. This form will allow the researcher to contact your carer directly to inform them of the project. If you do not complete this sheet, your carer will not be contacted regarding participation in the research.

Although you may nominate someone, there participation is also voluntary, therefore they will decide if they would like to participate. It is worth considering that this may occur if you decide to nominate someone. 


\section{Are there possible disadvantages of taking part?}

Although the research will not focus upon your life experiences, we will be talking about your experiences of Woodlands, which could be positive and negative, and this may naturally progress onto the current challenges you are facing and about your recovery. If you have had any difficult experiences these may make you feel upset or angry, you will be able to stop the interview at any point.

\section{What are the possible benefits of taking part?}

As the research is an evaluation it gives you the opportunity to have your voice heard in relation to the treatment and services Woodlands currently offers. Although it is important to note that all participation is voluntary and there will be no consequences if you decide not to participate.

\section{Will my taking part in this project be kept confidential?}

All information which is collected about you during the research project will be kept strictly confidential, and only Amy will have access to the data as it will be kept on a password-protected computer. This information refers to the consent forms you complete, the audio recordings of the interview, the routine questionnaire data already collected at Woodlands and the typed-up version of your interview. All information from the research project will be destroyed after 5 years.

On occasions, Amy may need to share information with her supervisory team, these are staff members at Swansea University, however, this information will not contain any information that could identify you. Some direct quotations from interviews may be reported within my PhD thesis and other publications but these will be linked with the pseudonym (fake name) you pick. Individual comments that you make during the interview will not be passed onto staff at Woodlands unless there are any concerns about a risk to yourself or others. If this does happen, Amy will need to share these concerns with the team at the Woodlands so they can support you.

\section{What is a Pseudonym and why is it necessary?}

A pseudonym is a fictional name assigned to give anonymity to a person, group, or place. This is a way to ensure that no one can be identified through the research.

\section{What will happen to the results of the research project?}

Results will be presented at conferences, written up for journal articles and will be part of the write up of Amy's PhD thesis. If any of your individual data is presented and/or published, the data will be totally anonymous, therefore there will be no means of identifying you as the individual involved.

\section{What if I decide I no longer want to take part in the research project?}

You are free to change your mind and withdraw from the research at any time until November 2019, which is at the end of the data collection period. All you need to do is contact Amy and she will remove all the information you have provided from the data.

\section{Who is organising the research?}


Amy Pritchard is a PhD student at Swansea University who will be independently evaluating Woodlands and two interventions it offers. Although Woodlands approached Swansea University for this project, the data collection and analysis will be completely independent of Woodlands.

\section{Who has reviewed this project?}

REC [Wales Research Ethics Committee 6]

\section{Contact for further information}

If you have any questions relating to this project, then please contact the Chief Investigator on j.y.rance@swansea.ac.uk. If you do not have internet access contact Woodlands to contact the researcher on your behalf and Amy will respond.

\section{How to make a complaint regarding the research}

If you are unhappy with the way in which your personal data has been processed you may in the first instance contact the University Data Protection Officer using the contact details:

University Compliance Officer (FOI/DP) Vice-Chancellor's Office,

Swansea University, Singleton Park,

Swansea,

SA2 8PP

Email: dataprotection@swansea.ac.uk

If you remain dissatisfied then you have the right to apply directly to the Information Commissioner for a decision. The Information Commissioner can be contacted at: -

Information Commissioner's Office,

Wycliffe House,

Water Lane,

Wilmslow,

Cheshire,

SK9 5AF

www.ico.org.uk

How to make a complaint regarding Woodlands

If you are unhappy with regards to the Woodlands, you can make a complaint to:

Healthcare Inspectorate Wales ,

Welsh Government, Rhydycar Business Park, Merthyr Tydfil,

CF48 $1 \mathrm{UZ}$

Tel: 03000628163
Healthcare Inspectorate Wales, Llywodraeth Cymru, Parc Busnes Rhydycar, Merthyr Tudful, CF48 $1 \mathrm{UZ}$ Ffon:0300 0628163 


\section{Service-User Consent Form - A Realist Evaluation of Woodlands}

Please read and complete this form carefully before placing your initials in each of the box. If you are willing to participate in this study, please complete this form and sign and date the declaration at the end. If you do not understand anything and would like more information, please ask.

- I confirm that I have read and fully understand the participant information sheet provided to me for this study and have had the opportunity to ask any questions.

- I understand that participation in this study is voluntary and I may withdraw from this study at any time without having to give an explanation by contacting the researcher, Amy Pritchard. I am aware that withdrawing from the project will not affect my current care or treatment at Woodlands.

- I give consent for my data, including direct quotations, to be used anonymously for Academic research purposes including journal publications, presentation at academic conferences and in the write up of the researcher's PhD thesis. I give consent to my data being analysed in various ways.

- I understand that the audiotape material from the interview will be used solely for research purposes and will be destroyed once it has been transcribed.

- I give permission for the routine data collected at Woodlands to be used for the purposes of this research.

- I understand that the researcher, Amy, will be discussing the progress of the research with her supervisor at Swansea University.

- I give consent for the researcher, Amy Pritchard, to contact the individual I have nominated to be invited to participate in this research.

I freely give my consent to participate in this research study and have been given a copy of this form for my own information.

Participant Name :

Participant Signature:

Date:

Researcher Signature:

If you have any questions or wish to withdraw for the study, please contact the Chief Investigator on the following email address: on j.y.rance@swansea.ac.uk Please include your pseudonym (fake name) so you can be removed from the data. Thank you. 
Appendix G - Service-user Debrief Form

A Realist Evaluation of Woodlands - Debrief After Interviews

Thank you for taking part in data collection today. Your time and effort throughout this research project has been hugely appreciated.

If you have any queries about this study, please do not hesitate to contact the Chief Investigator using the email address provided below. As all data collection is anonymous if you would like your data to be removed from the study you will need to provide your study identifier which is the pseudonym (fake name) you chose for the interview.

Chief Investigator - Jaynie Rance - on j.y.rance@swansea.ac.uk

If you cannot email directly, ask a member of staff at Woodlands to contact the Chief Investigator on your behalf. The Chief Investigator or a member of the research team will then respond to you either via telephone communication or face-to-face at Woodlands. If you do ask staff to contact the research team on your behalf, please do not share with them your reasons so that confidentiality can be maintained.

\section{Useful Numbers and Resources}

If you feel as though you would like extra support after taking part in this research, please contact:

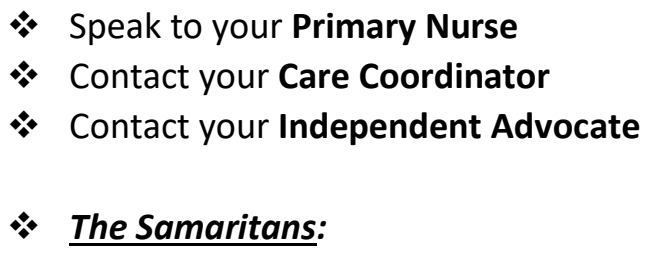

Telephone Number: 116123 (this telephone number is free of charge)

Samaritans telephone line is open 24 hours a day, 365 days a year, to listen to anything that is upsetting you. Samaritans also offer a Welsh Language Line on 03001233011 (from 7pm-11pm only, seven days a week.

Website: $h t t p: / / w w w . s a m a r i t a n s . o r g /$ 


\section{Consent for Contacting Carer}

Please read and complete this form carefully before placing your initials in the box. If you are willing for the researcher to contact your carer to invite them to participate in this study, please complete this form and sign and date the declaration at the end. If you are happy, then please provide their name and contact information of your identified carer below. If you do not understand anything and would like more information, please ask.

Please be mindful that the participation of your carer is voluntary and it is their decision if they would like to participate in this research. Your nomination does not guarantee that the carer will consent and participate in this project.

I give my consent for the researcher to contact my carer so that they can be invited to participate in this research.

Name of Carer:

\section{Address:}

I freely give my consent for the researcher to contact my carer regarding this research study and have been given a copy of this form for my own information.

Participant Name :

Participant Signature:

Date:

Researcher Signature:

If you have any questions or wish to withdraw for the study, please contact the Chief Investigator on j.y.rance@swansea.ac.uk please include your pseudonym (fake name) so you can be identified and removed. 


\section{Letter to Carers to Inform them of the Project}

Dear [insert name],

[insert date]

I am writing to inform you about a research project that is currently underway at Woodlands. Woodlands has asked me, a researcher from Swansea University, to independently evaluate the service, to look at what is and is not working and the impact of the work they are currently doing. For me to do this I would like to listen to as many opinions as possible and would really value the input of carers.

You have been identified by a current client at Woodlands as their carer and they have consented for me to contact you directly to invite you to consider participating in this project. Participation in this project is voluntary and therefore if you decide not to participate this will not impact your current involvement with the individual at Woodlands or the team. A participant information sheet has also been included in this letter to inform you more about the research, what is required of participants and how your comments will be kept confidential, secure and anonymous. If you decide to participate in this project, you will be required to participate in a 30-minute interview with the researcher to give your opinions on the treatment and care provided at Woodlands and to understand your experience from the perspective of a carer.

If you would like to contact me directly you can email the Chief Investigator on or you can provide the clinical team at Woodlands with your contact number and consent to be contacted and I will call you to discuss the research.

Thank you for taking the time to read this letter.

Best wishes,

Amy Pritchard.

PhD Researcher at Swansea University.

Swansea University, Singleton Campus, Vivian Tower. $3^{\text {rd }}$ Floor Postgraduate Office. SA2 8PP. 


\section{Appendix J - Carer Participant Information Sheet}

\section{$\underline{\text { A Realist Evaluation of Woodlands - Carer Participant Information Sheet }}$}

I would like to invite you to take part in a research project. Before you decide you need to understand why the research is being done and what it will involve for you as a potential participant. Please take your time to read the following information and to decide if you would like to participate in this research project. If you have any questions then please ask the key researcher, Amy.

\section{Why is this research being done?}

- The aim of this project is to evaluate how Woodlands supports individuals in their mental health recovery.

- The aim is to understand what is and is not currently working at Woodlands and to understand why and how it is or is not working.

\section{Why have I been chosen?}

You have been nominated as you are a carer to a current client at Woodlands and will be able to provide valuable information to enable the researcher to evaluate the services available at Woodlands.

\section{Do I have to take part?}

This research is completely voluntary and will not affect the care of the client at Woodlands. You will be provided with all the information about the project first and given some time to consider whether you would like to participate. You will then be asked to read and sign a consent form to show you have voluntarily agreed to take part in the research. You are free to change your mind and withdraw from the research at any time until December 2019, which is the end of the data collection period.

\section{What do I have to do if I agree to be part of the research project?}

You will be invited to take part in an interview with Amy, which will last up to 30 minutes. All the interviews will be audio recorded, but the audio tapes will be destroyed once they have been typed up by Amy.

\section{Are there possible disadvantages and/or risks of taking part?}

Although the research will not focus upon your life experiences, we will be talking about your experiences of Woodlands, which could be positive and negative, and this may naturally progress onto the individual's current challenges and about their mental health recovery. If you have had any difficult experiences these may make you feel upset or angry, the researcher will support you and you are able to stop the interview at any point.

\section{What are the possible benefits of taking part?}

As the research is an evaluation it gives you the opportunity to have your voice heard in relation to the treatment and services Woodlands currently offers. Although it is important to note that all participation is voluntary and there will be no consequences if you decide not to participate. 


\section{Will my taking part in this project be kept confidential?}

All information which is collected about you during the research project will be kept strictly confidential, and only the key researcher will have access to the data as it will be kept on a password-protected computer or a locked cabinet. On occasions, the key researcher may need to share information with her supervisory team, however, your identity will remain anonymous. All information from the research project will be destroyed after 5 years.

Individual comments that you make during the interview will not be disclosed to the staff at Woodlands unless there are any concerns about a risk to or the treatment of the individual at Woodlands or others. If this does occur, the researcher will have to breach confidentiality and share this with the Healthcare Wales Inspectorate if appropriate.

\section{What is a Pseudonym and why is it necessary?}

A pseudonym is a fictional name assigned to give anonymity to a person, group, or place. This is a way to ensure that no one can be identified through the research.

\section{What will happen to the results of the research project?}

Results will be presented at conferences, written up for journal articles and will be part of the write up of the key researcher's PhD thesis. If any of your individual data is presented and/or published, the data will be totally anonymous, therefore there will be no means of identifying you as the individual involved.

\section{Who is organising the research?}

Amy Pritchard is a PhD student at Swansea University who will be independently evaluating three interventions that Woodlands offer. Woodlands approached Swansea University to work collaboratively on this project, however, the data collection and analysis will be completely independent of Woodlands.

\section{Who has reviewed this project?}

REC [Wales Research Ethics Committee 6]

\section{Contact for further information}

If you have any questions relating to this project, then please contact the Chief Investigator on j.y.rance@swansea.ac.uk. If you do not have internet access contact Woodlands to contact the researcher on your behalf and the researcher will respond to this either via telephone communication or face-to-face communication at Woodlands.

\section{How to make a complaint regarding the research}

If you are unhappy with the way in which your personal data has been processed you may in the first instance contact the University Data Protection Officer using the contact details:

University Compliance Officer (FOI/DP) Vice-Chancellor's Office

Swansea University, Singleton Park 
Swansea

SA2 8PP

Email: dataprotection@swansea.ac.uk

If you remain dissatisfied then you have the right to apply directly to the Information Commissioner for a decision. The Information Commissioner can be contacted at: -

Information Commissioner's Office,

Wycliffe House,

Water Lane,

Wilmslow,

Cheshire,

SK9 5AF

www.ico.org.uk

\section{How to make a complaint regarding Woodlands}

If you are unhappy with regards to the Woodlands, you can make a complaint to:

Healthcare Inspectorate Wales,

Welsh Government,

Rhydycar Business Park,

Merthyr Tydfil,

CF48 1UZ

Tel: 03000628163
Healthcare Inspectorate Wales, Llywodraeth Cymru, Parc Busnes Rhydycar, Merthyr Tudful,

CF48 1 UZ

Ffon:0300 0628163 


\section{$\underline{\text { A Realist Evaluation of Woodlands - Consent Form for Carers }}$}

Please read and complete this form carefully before placing your initials in each of the box. If you are willing to participate in this study, please complete this form and sign and date the declaration at the end. If you do not understand anything and would like more information, please ask.

- I confirm that I have read and fully understand the participant information sheet provided to me for this study and have had the opportunity to ask any questions.

- I understand that participation in this study is voluntary and I may withdraw from this study at any time without having to give an explanation by contacting the researcher, Amy Pritchard. I am aware that withdrawing from the project will not affect my involvement with the person you care for or the team at Woodlands.

- I give consent for my data, including direct quotations, to be used anonymously for Academic research purposes including journal publications, presentation at academic conferences and in the write up of the researcher's PhD thesis.

- I understand that the researcher, Amy, will be discussing the progress of the research with her supervisor at Swansea University.

I freely give my consent to participate in this research study and have been given a copy of this form for my own information.

Participant Name :

Participant Signature:

Date:

Researcher Signature:

If you have any questions or wish to withdraw for the study, please contact the Chief Investigator on please include your pseudonym (fake name) so you can be identified and removed.

Thank you. 
Appendix L - Carer Debrief Sheet

\section{Carer Debrief After Interviews}

Thank you for taking part in this interview today. Your time and effort throughout this research project has been hugely appreciated.

If you have any queries about this study, please do not hesitate to contact Amy using the email address provided below. As all data collection is anonymous if you would like your data to be removed from the study you will need to provide Amy with the pseudonym (fake name) you chose for the interview.

Amy Pritchard - Email Address

If you do not have internet access to contact Amy directly then you can contact Woodlands and provide them with an alternative means of Amy contacting you, such as a phone number. Woodlands will then contact Amy on your behalf, providing her with your contact information. If you do contact Woodlands, please do not tell the Woodlands the reason for needing contact with Amy to maintain confidentiality and anonymity.

\section{Useful Numbers and Resources}

If you feel as though you would like extra support after taking part in this research, please contact:

\section{- Carers Trust Wales:}

Telephone Number: 02920090087

E-mail: wales@carers.org

Website: https://carers.org/country/carers-trust-wales-cymru

\section{* Carers UK Advice Line:}

Telephone Number: 080807777

Open Mondays and Tuesdays 10am to 4pm

\section{* The Samaritans:}

Telephone Number: 116123 (this telephone number is free of charge)

Samaritans telephone line is open 24 hours a day, 365 days a year, to listen to anything that is upsetting you. Samaritans also offer a Welsh Language Line on 03001233011 (from 7pm-11pm only, seven days a week.

Website: http://www.samaritans.org/ 


\section{Topic Guide for Programme Architect Interviews -}

\section{Introduction:}

Thank the participant for agreeing to the interview. Check to ensure the participant is aware of the purpose of the research, has given informed consent and is aware and OK with the interview being recorded. Ask them if they have any questions before the interview begins.

Explain that you are here to talk to them because of their role in the development of a [specific intervention] delivered at Woodlands.

\section{Topics to be covered during the Realist Interview:}

\section{Context:}

Questions will relate to the specific details about the individuals who deliver the intervention, the recipients of the intervention, the wider culture of the service and organisation and the interpersonal relationships that might impact upon the way in which the intervention works. The aim of these questions will be to determine the pre-existing factors that make up the environment in which the intervention has been introduced. The programme architect will then be asked how they feel the context provides an environment that supports or hinders the intervention being delivered.

For example:

- Why was this service considered necessary?

- What subgroup of clients is Woodlands aiming to support?

- Can you tell me about the barriers and facilitators to the successful delivery of Woodlands?

\section{Mechanisms:}

The questions relating to the mechanisms of the intervention consist of two parts:

Questions will relate to the specific resources the intervention provides the recipients, such as the information or skills the recipients will receive because of engaging in the intervention. The programme architect will be questioned on their decisions to provide these resources and what they expected the recipients to get from these resources.

For example:

- What resources are provided at Woodlands to support individuals in their recovery?

- How are these resources delivered at Woodlands?

The second part of the mechanism relates to the recipient response. This is the cognitive or emotional response that recipients have to the intervention or the resources it provides. The programme architect will be questioned about how they believe recipients will think or feel in response to the intervention.

For example: 
- What emotional responses would you expect to see from clients who engage with the [insert resource]?

\section{Outcomes:}

The questions relating to the outcomes of the intervention will relate to the expected and unexpected client outcomes and will aim to identify narratives that demonstrate the impact the intervention has had at Woodlands.

For example:

- What outcomes would you expect if an individual engaged with this resource?

\section{Ending the Interview:}

- Is there anything else you'd like to say?

- What is the message you'd like me to really take away today?

Close the interview and thank the participant for their participation. 


\section{Appendix N - Topic Guide for Staff}

\section{Topic Guide for Staff Interviews}

\section{Introduction:}

Thank the participant for agreeing to the interview. Check to ensure the participant is aware of the purpose of the research, has given informed consent and is aware and OK with the interview being recorded. Ask them if they have any questions before the interview begins.

Explain that you are here to talk to them because of their role in delivering [specific intervention] at Woodlands.

\section{Topics to be covered during the Realist Interview:}

\section{Context:}

Questions will relate to the specific details about the individuals who deliver the intervention, the recipients of the intervention, the wider culture of the service and organisation and the interpersonal relationships that might impact upon the way in which the intervention works. The aim of these questions will be to determine the pre-existing factors that make up the environment in which the intervention has been introduced. The aim of staff interviews will be to test if the programme is working as intended and to see what the facilitators have experienced in practice.

For example:

- Was the resource of [insert resource] suitable for the clients at Woodlands?

- Can you tell me about the barriers and facilitators to the successful delivery of Woodlands?

\section{Mechanisms:}

The questions relating to the mechanisms of the intervention consist of two parts:

Questions will relate to the specific resources the intervention provides the recipients, such as the information or skills the recipients will receive. The facilitators will be asked if there are aspects of the intervention that, in practice, seem to work better and why this was the case.

For example:

- Relating to resource of [insert resource] what information or skills did guests develop?

The second part of the mechanism relates to the recipient response. This is the cognitive or emotional response that recipients have to the intervention or the resources it provides. The facilitators will be asked if there were any noticeable changes in recipients' responses to the intervention and resources.

For example:

- What emotional responses would you expect to see within guests who engage with the resource of [inset resource] at Woodlands?

\section{Outcomes:}

The questions relating to the outcomes of the intervention will relate to the expected and unexpected client outcomes and will aim to identify narratives that demonstrate the impact the intervention has had at Woodlands. 
For example:

- Do you have any stories that demonstrate the success of the Woodlands?

\section{Ending the Interview:}

- Is there anything else you'd like to say?

- What is the message you'd like me to really take away today?

Close the interview and thank the participant for their participation. 


\section{Appendix O - Service-user Topic Guide}

\section{$\underline{\text { Topic Guide for Client Interviews }}$}

\section{Introduction:}

Thank the participant for agreeing to the interview. Check to ensure the participant is aware of the purpose of the research, has given informed consent and is aware and OK with the interview being recorded. Ask them if they have any questions before the interview begins.

Explain that you are here to talk to them because of being a recipient of the [specific intervention] being evaluated at Woodlands.

Topics to be covered during the Realist Interview: The client interviews have been amended so that they are shorter and less of a burden on participants. They will cover the areas that the client will be able to provide the richest data on. The main aim of interviews with clients is to determine outcomes and satisfaction of the intervention being evaluated.

\section{Mechanism:}

The questions relating to the mechanisms of the intervention consist of two parts:

Questions will relate to the specific resources the intervention provides the recipients, such as the information or skills the recipients will receive. The recipients will be asked if there were any areas of the intervention that were better compared to others and why.

For example:

- What did you like, if anything, about Woodlands?

The second part of the mechanism relates to the recipient response. This is the cognitive or emotional response that recipients have to the intervention or the resources it provides. The recipient will be asked if the intervention made them think or feel any different afterwards and if this impacted upon their behaviour as a result.

For example:

- How does [insert resource] make you feel?

\section{Outcomes:}

The questions relating to the outcomes of the intervention will relate to the impact the intervention had on the client from the client's perspective.

For example:

- What have you learnt from being at Woodlands?

\section{Ending the Interview:}

- Is there anything else you'd like to say?

- What is the message you'd like me to really take away today?

Close the interview and thank the participant for their participation. 


\section{$\underline{\text { Topic Guide for Carer Interviews }}$}

\section{Introduction:}

Thank the participant for agreeing to the interview. Check to ensure the participant is aware of the purpose of the research, has given informed consent and is aware and OK with the interview being recorded. Ask them if they have any questions before the interview begins.

Explain that you are here to talk to them because of being a carer for someone at Woodlands.

Topics to be covered during the Realist Interview: The carer interviews have been amended so that they are shorter and less of a burden on participants. They will cover the areas that the carer will be able to provide the richest data on. The main aim of interviews with carers is to determine outcomes and satisfaction of the service Woodlands provides.

\section{Outcomes:}

The questions relating to outcomes will relate to the changes carers have seen within the individual at Woodlands and if they attribute these changes to anything that Woodlands specifically offers. Also, the questions will aim to identify their experience of attending/working with the care team at Woodlands.

For example:

- Have you noticed any changes in the person you care for during their stay at Woodlands?

\section{Ending the Interview:}

- Is there anything else you'd like to say?

- What is the message you'd like me to really take away today?

Close the interview and thank the participant for their participation. 


\section{ReQoL

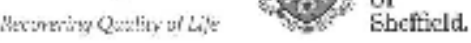

For each of the following statements, please tick one box that best describes your thoughts, feelings and activities over the last week.

\begin{tabular}{|c|c|c|c|c|c|}
\hline Over the last week & $\begin{array}{l}\text { None } \\
\text { of the time }\end{array}$ & $\begin{array}{c}\text { Only } \\
\text { occasionally }\end{array}$ & Sometimes & Often & $\begin{array}{l}\text { Most or all } \\
\text { of the time }\end{array}$ \\
\hline $\begin{array}{l}\text { 1. I found it difficult to get sta } \\
\text { with everyday tasks }\end{array}$ & & & & & \\
\hline 2. I felt able to trust others & & & & & \\
\hline 3. I felt unable to cope & & & & & \\
\hline $\begin{array}{l}\text { 4. I could do the things I want } \\
\text { to do }\end{array}$ & & & & & \\
\hline 5. I felt happy & & & & & \\
\hline $\begin{array}{l}\text { 6. I thought my life was not } w \\
\text { living }\end{array}$ & & & & & \\
\hline 7. I enjoyed what I did & & & & & \\
\hline 8. I felt hopeful & & & & [ & \\
\hline 9. I felt lonely & & & & & \\
\hline 10. I felt confide & ] & & & & 1 \\
\hline & $\begin{array}{l}\text { No } \\
\text { problems }\end{array}$ & $\begin{array}{l}\text { Slight } \\
\text { problems }\end{array}$ & $\begin{array}{l}\text { Moderate } \\
\text { problems }\end{array}$ & $\begin{array}{l}\text { Severe } \\
\text { problems }\end{array}$ & $\begin{array}{l}\text { Very severe } \\
\text { problems }\end{array}$ \\
\hline $\begin{array}{l}\text { Please describe your physical } \\
\text { health (problems with pain, } \\
\text { mobility, difficulties caring for } \\
\text { yourself or feeling physically } \\
\text { unwell) over the last week }\end{array}$ & & & & & \\
\hline
\end{tabular}

For official use

ReQoL-10 Score =

ReQol- Vervion 1.10 Copyright, The University of Shetrield 2016, 2018. All Rights Reserved. The suthors have asserted their moral rights. Ouford University Innovation Limited is exclusively ficensed to frant permissions to use the ReQoL"-

ReQol-10 English for United Kingdom. 


\section{VOICE}

\section{Views On Inpatient Care}

\section{Service Users' Perceptions Questionnaire}

Developed through the PERCEIVE programme (NIHR) www.perceive.iop.kcl.ac.uk

\section{CONFIDENTIAL}

Your answers on this questionnaire will be confidential and will not be shown to any of the staff on this ward.

Please give answers based on your experiences on this ward.

We know that individual staff can vary, but try to think about the majority of staff and come up with an average answer.

Please tick one answer per question.

\section{percelve}

(c) 2010 King's College London

$-1-$ 


\section{ADMISSION}

1 I was made to feel welcome when I arrived on this ward.

○

0

○

○

○

○

Strongly

Agree

Slightly

Agree

Slightly

Disagree

Disagree

Strongly

Agree

Any comments

\section{CARE AND TREATMENT}

2 I have a say in my care and treatment.

O

O

O

O

O

Strongly
Agree

Agree

Slightly

Agree

Slightly

Disagree

Disagree

Disagree

3 Ward rounds are useful for me.

O

O

O

O

0

O

Strongly

Agree

Slightly

Agree

Slightly

Disagree

Disagree

Strongly

Agree

Any comments

\section{MEDICATION}

4 I feel my medication helps me.

O

o

o

0

o

O

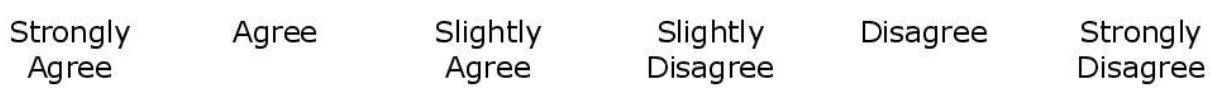

() 2010 King's College London 
$5 \quad$ I have the opportunity to discuss my medication and side effects.

$\begin{array}{cccccc}0 & 0 & 0 & 0 & 0 & 0 \\ \begin{array}{c}\text { Strongly } \\ \text { Agree }\end{array} & \text { Agree } & \begin{array}{c}\text { Slightly } \\ \text { Agree }\end{array} & \begin{array}{c}\text { Slightly } \\ \text { Disagree }\end{array} & \text { Disagree } & \begin{array}{c}\text { Strongly } \\ \text { Disagree }\end{array}\end{array}$

6 Staff give me medication instead of talking to me.
O
O
O
O
O
$\mathrm{O}$

\begin{tabular}{|c|c|c|c|}
\hline $\begin{array}{c}\text { Strongly } \\
\text { Agree }\end{array}$ & Agree & $\begin{array}{c}\text { Slightly } \\
\text { Agree }\end{array}$ & $\begin{array}{c}\text { Slightly } \\
\text { Disagree }\end{array}$ \\
\hline
\end{tabular}

\section{Any comments}

\section{STAFF}

$7 \quad$ Staff take an interest in me.
$\mathrm{O}$
O
O
O
O
O

\begin{tabular}{|c|c|c|c|}
\hline $\begin{array}{c}\text { Strongly } \\
\text { Agree }\end{array}$ & Agree & $\begin{array}{c}\text { Slightly } \\
\text { Agree }\end{array}$ & $\begin{array}{l}\text { Slightly } \\
\text { Disagree }\end{array}$ \\
\hline
\end{tabular}

8 Staff are available to talk to when I need them.

O

O

O

O

O

$\mathrm{O}$

\begin{tabular}{|c|c|c|c|c|}
\hline $\begin{array}{c}\text { Strongly } \\
\text { Agree }\end{array}$ & Agree & $\begin{array}{c}\text { Slightly } \\
\text { Agree }\end{array}$ & $\begin{array}{c}\text { Slightly } \\
\text { Disagree }\end{array}$ & Disagree \\
\hline
\end{tabular}

$9 \quad$ I trust the staff to do a good job.
O
O
O
O
O
O

\begin{tabular}{|c|c|c|c|c|}
\hline $\begin{array}{c}\text { Strongly } \\
\text { Agree }\end{array}$ & Agree & $\begin{array}{c}\text { Slightly } \\
\text { Agree }\end{array}$ & $\begin{array}{l}\text { Slightly } \\
\text { Disagree }\end{array}$ & Disagree \\
\hline
\end{tabular}

(c) 2010 King's College London 
10 I feel that staff understand how my illness affects me.

O

Strongly

Agree
O

Slightly

Agree
O

Slightly Disagree
O

Disagree

Strongly Disagree

11 I feel that staff treat me with respect.

O

$$
\begin{aligned}
& \text { Strongly } \\
& \text { Agree }
\end{aligned}
$$

O Slightly
Agree
O

Slightly Disagree o

Disagree
O

Strongly Disagree

\section{Any comments}

\section{THERAPY AND ACTIVITIES}

12 I think the activities on the ward meet my needs.

O

Strongly Agree o

Agree

Agree
O

Slightly Agree
O

Slightly Disagree
$\mathrm{O}$

Disagree

Strongly Disagree

13 I find one-to-one time with staff useful.

O

Strongly Agree
O Slightly
Agree
O

Slightly Disagree
O

Disagree
O

Strongly Disagree

\section{Any comments}




\section{ENVIRONMENT}

14 I find it easy to keep in contact with family and friends when I'm on the ward.

O

O

O

O

O

O

\begin{tabular}{|c|c|c|c|}
\hline $\begin{array}{c}\text { Strongly } \\
\text { Aqree }\end{array}$ & Agree & $\begin{array}{c}\text { Slightly } \\
\text { Aqree }\end{array}$ & $\begin{array}{c}\text { Slightly } \\
\text { Disagree }\end{array}$ \\
\hline
\end{tabular}

15 I feel safe on the ward.

$\mathrm{O}$

$\mathrm{O}$

O

O

O

\begin{tabular}{|c|c|c|c|}
\hline $\begin{array}{c}\text { Strongly } \\
\text { Agree }\end{array}$ & Agree & $\begin{array}{c}\text { Slightly } \\
\text { Agree }\end{array}$ & $\begin{array}{l}\text { Slightly } \\
\text { Disagree }\end{array}$ \\
\hline
\end{tabular}

16 I feel staff respond well when the panic alarm goes off.

O

O

O

O

O

Strongly Agree Slightly Slightly Disagree Strongly

17 I feel staff respond well when I tell them I'm in crisis.

O

O

O

O

0

$\mathrm{O}$

\begin{tabular}{|c|c|c|c|}
\hline $\begin{array}{c}\text { Strongly } \\
\text { Aaree }\end{array}$ & Agree & Slightly & Slightly \\
\hline
\end{tabular}

Any comments

(C) 2010 King's College London 


\section{DIVERSITY}

18 I feel able to practice my religion whilst $I^{\prime} m$ in hospital.

O

O

O

$\mathrm{O}$

O

O

Strongly

Agree

Agree

Slightly

Agree

Slightly

Disagree

Disagree

Strongly Disagree

19 I think staff respect my ethnic background.

O

Strongly

Agree

Agree

O

O

O

O

O

Any comments

(C) 2010 King's College London 


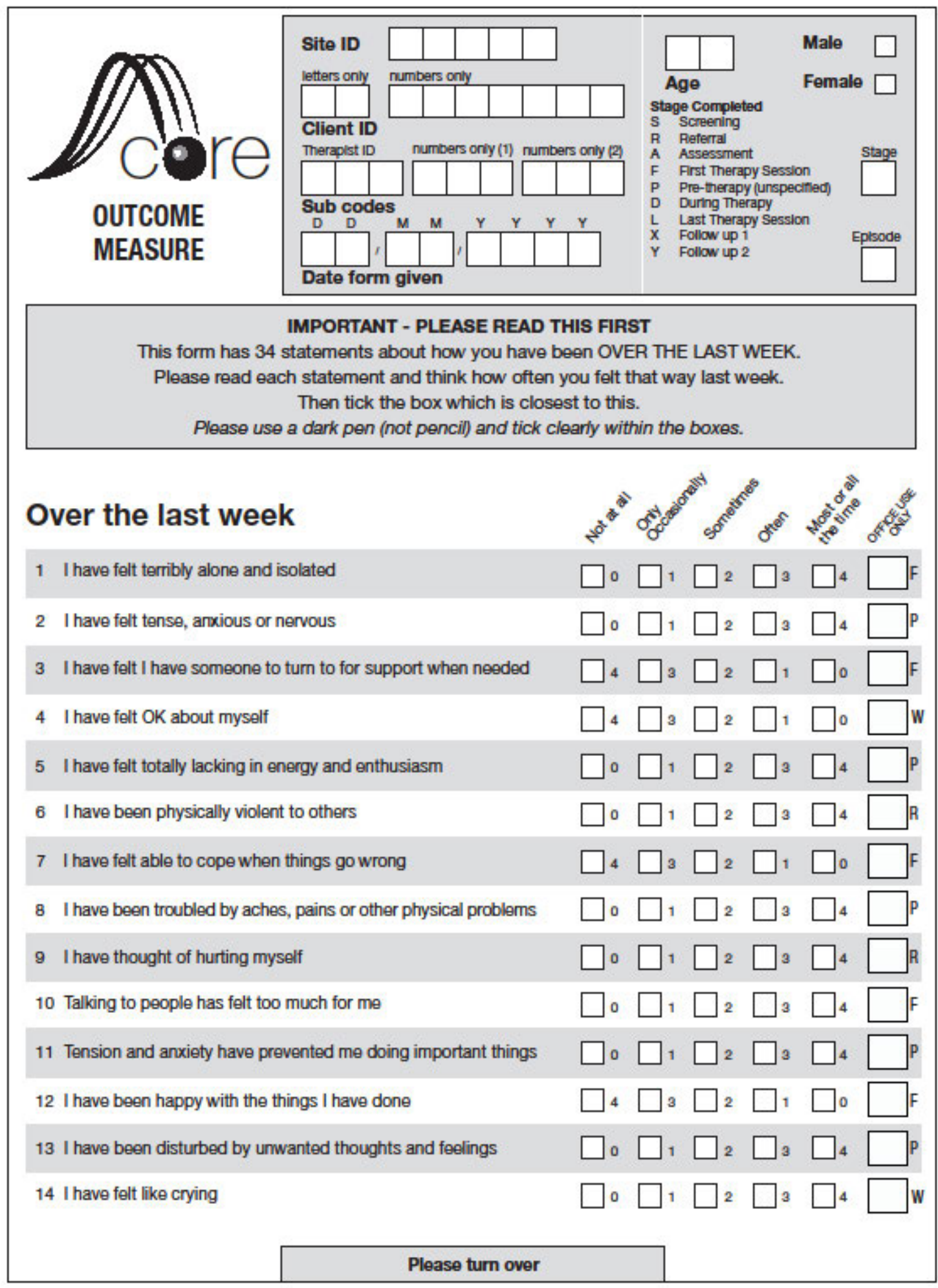

survoy. 151

Q CORE System Trust: http://www.coraims.co.uk/copyright.pdf

Fago: 1 Supported by www.coreims.co.uk 


\section{Over the last week}

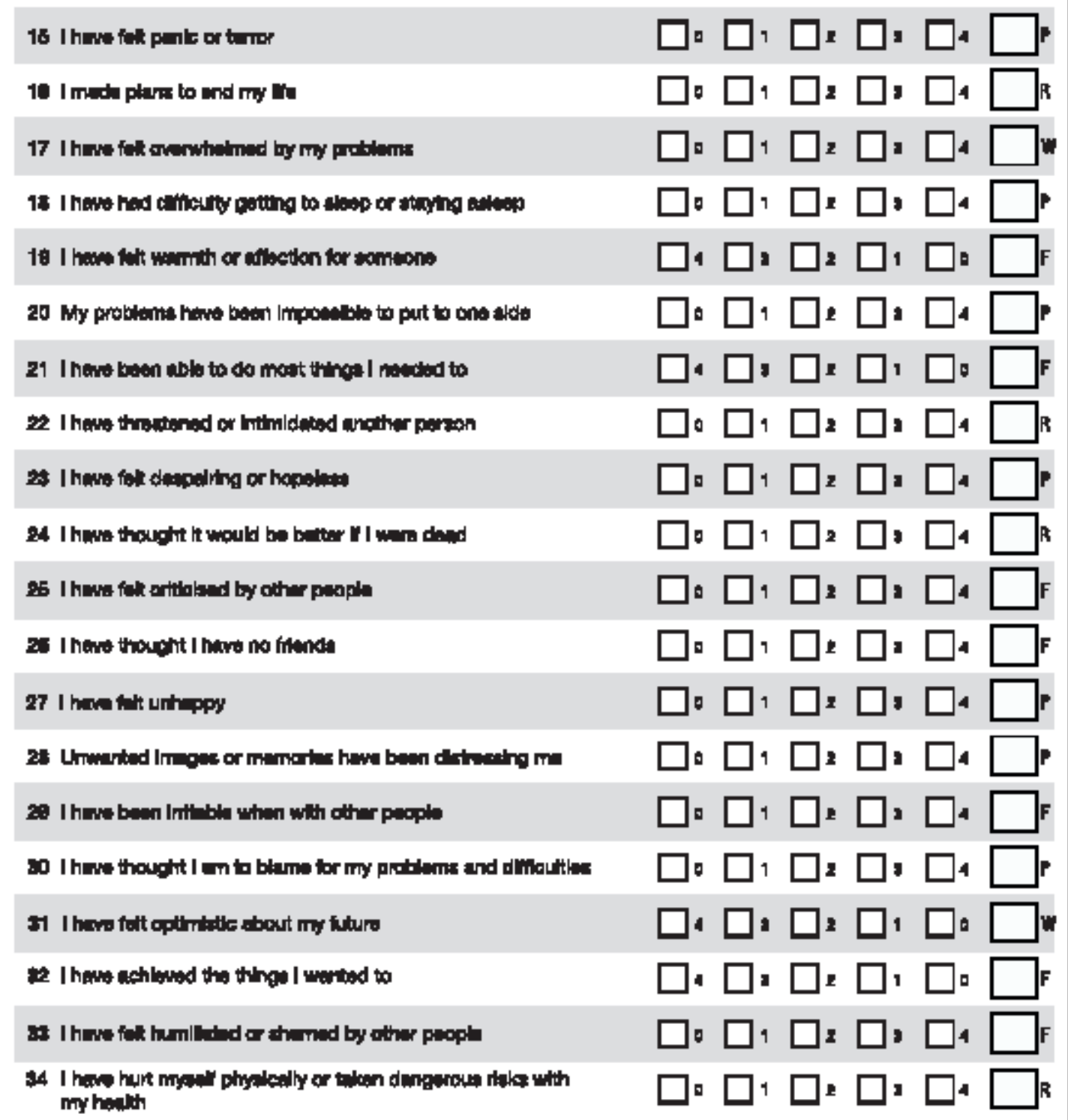

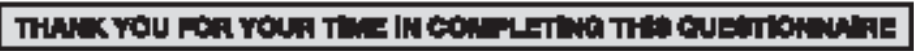

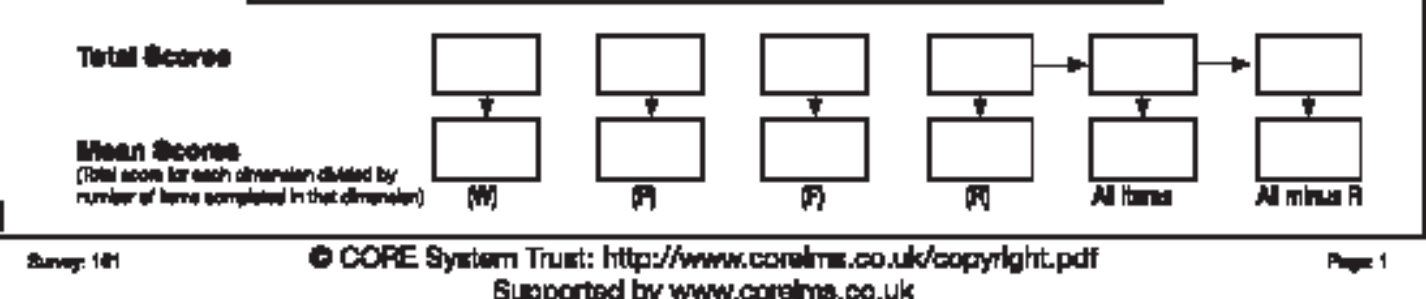


Appendix T - Framework Approach

\begin{tabular}{|c|c|}
\hline Initial Codes & $\begin{array}{c}\text { Overarching Categories used within } \\
\text { Framework }\end{array}$ \\
\hline Referrals & \multirow[b]{2}{*}{$\begin{array}{l}\text { This category related to the difficulties the } \\
\text { service experienced in securing referrals. This } \\
\text { considered any reasons behind the troubles in } \\
\text { securing referrals and the influence this had in } \\
\text { practice. }\end{array}$} \\
\hline $\begin{array}{l}\text { C Caps System / NHS } \\
\text { Framework } \\
\text { Low service-user numbers }\end{array}$ & \\
\hline Training / capabilities & \multirow{3}{*}{$\begin{array}{l}\text { This category related to the staffing group } \\
\text { working at Woodlands and how they } \\
\text { supported service-users in their recovery. }\end{array}$} \\
\hline Staffing Levels & \\
\hline Peer Mentors & \\
\hline \multirow[t]{2}{*}{ Daily Living Tasks } & \multirow{3}{*}{$\begin{array}{l}\text { This category related to factors where the } \\
\text { service-user was expected to take } \\
\text { responsibility for daily living tasks as these } \\
\text { skills were considered necessary for } \\
\text { community living. }\end{array}$} \\
\hline & \\
\hline Skill Development & \\
\hline Choice about Activities & \multirow{7}{*}{$\begin{array}{l}\text { This category related to the types of decisions } \\
\text { service-users made whilst at Woodlands. } \\
\text { Service-user choice was discussed in terms of } \\
\text { discussions about what service-users wanted } \\
\text { to do, the community meeting and how } \\
\text { importance was placed upon service-user's } \\
\text { ability and desire to accept the responsibility } \\
\text { that comes with choice. }\end{array}$} \\
\hline $\begin{array}{l}\text { Accessing community-based } \\
\text { opportunities }\end{array}$ & \\
\hline Choices about Treatment & \\
\hline $\begin{array}{l}\text { Choice about Carer } \\
\text { Involvement }\end{array}$ & \\
\hline Community Meeting & \\
\hline $\begin{array}{l}\text { Choice as a learning } \\
\text { opportunity }\end{array}$ & \\
\hline Positive risk-taking to learn & \\
\hline
\end{tabular}




\begin{tabular}{|c|c|}
\hline Physical Environment & $\begin{array}{c}\text { Physical Environment } \\
\text { This category related to the physical design of } \\
\text { the service and how this created a safe and } \\
\text { comfortable environment for recovery. }\end{array}$ \\
\hline Wider Organisation & $\begin{array}{c}\text { Wider Organisation } \\
\text { This category related to the wider organisation } \\
\text { in which Woodlands operated and their } \\
\text { previous experiences of providing support for } \\
\text { service-users. }\end{array}$ \\
\hline Service-user criteria & $\begin{array}{c}\text { Intended Service-user Group } \\
\text { This category related to who Woodlands was } \\
\text { designed to support. }\end{array}$ \\
\hline
\end{tabular}

In practice, staff, service-users and commissioners made very little reference to the role of the physical environment and the wider organisation in supporting their recovery and therefore there was very little data relating to this and due to this I focused my attention on the more data rich categories of the framework. 


\section{Appendix U - Criteria for Determining Relevance of a Data Extract}

If the data extract fulfilled one or more of the following criteria, then it was considered as providing rich causal insights into how the service supported service-users in their mental health recovery. This was used to guide what exerts from the data transcripts were coded and how they were coded (either as a context, mechanism (resource and reasoning were separated) or an outcome). When using the term 'resource' this refers to an element that is mechanistically contributing to the workings of the service.

If the participant identified:

- a key resource that was specifically used to support service-users in their mental health recovery;

- how the key resource supported service-users in their mental health recovery;

- how service-users responded (cognitively or emotionally) to the key resource;

- how staff responded (cognitively or emotionally) to delivering the key resource;

- a service-user outcome from receiving a particular resource at the service;

- different responses or outcomes amongst the service-users at the service;

- an outcome of a particular resource at the service that impacted future service delivery;

- a staff outcome from delivering a particular resource at the service;

- a particular service-user characteristics that supported or hindered the success of a key resource supporting them within their mental health recovery (this could include from the perspective of the service-user or staff);

- a particular staff characteristics that supported or hindered the success of a key resource supporting service-users in their mental health recovery (this could include from the perspective of the service-user or staff);

- a particular service or organisational characteristics that supported or hindered the success of a key resource supporting clients in their mental health recovery (this could include from the perspective of the service-user or staff);

- a wider contextual factor that supported or hindered the success of a key resource supporting service-users in their mental health recovery (this could include from the perspective of the service-user or staff). 
Appendix V - Method Triangulation Table

\begin{tabular}{|c|c|c|}
\hline Key Findings & Interviews & Routinely Collected Data \\
\hline \multicolumn{3}{|l|}{ Wider Mental Health System } \\
\hline $\begin{array}{l}\text { The Service is } \\
\text { designed for } \\
\text { motivated, ambitious } \\
\text { service-users who } \\
\text { want to and are ready } \\
\text { to work on their } \\
\text { recovery }\end{array}$ & $\begin{array}{l}\text { Partial Agreement - } \\
\text { eventually Woodlands } \\
\text { accepted individuals with } \\
\text { personality disorder, } \\
\text { challenging behaviours. }\end{array}$ & $\begin{array}{l}\text { Partial Agreement - increase } \\
\text { in service-user thoughts of } \\
\text { self-harm, harming } \\
\text { themselves, having a suicide } \\
\text { plan and occasional violence } \\
\text { towards others. }\end{array}$ \\
\hline \multicolumn{3}{|l|}{ Choice and Responsibility } \\
\hline $\begin{array}{l}\text { - Service-users } \\
\text { encouraged to make } \\
\text { choices }\end{array}$ & $\begin{array}{l}\text { Partial Agreement - service- } \\
\text { users need to learn how to } \\
\text { make choices, staff could not } \\
\text { support certain choices, } \\
\text { activities need to be } \\
\text { meaningful, stigma from } \\
\text { community. }\end{array}$ & $\begin{array}{l}\text { Partial Agreement - activities } \\
\text { did not meet service-users' } \\
\text { needs or what they wanted, } \\
\text { service-users did not feel } \\
\text { they had a say. }\end{array}$ \\
\hline $\begin{array}{l}\text { Service-users take } \\
\text { responsibility by } \\
\text { completing Daily } \\
\text { Living Tasks }\end{array}$ & $\begin{array}{l}\text { Partial Agreement - staff } \\
\text { completed daily living tasks } \\
\text { on behalf of service-users. }\end{array}$ & $\begin{array}{l}\text { Partial Agreement - } \\
\text { struggled to start everyday } \\
\text { tasks. }\end{array}$ \\
\hline \multicolumn{3}{|l|}{ Staffing Model } \\
\hline $\begin{array}{l}\text { Staff will have people } \\
\text { skills and will be able } \\
\text { to work in a way } \\
\text { which moves beyond } \\
\text { focusing upon an } \\
\text { individual's mental } \\
\text { illness. }\end{array}$ & $\begin{array}{l}\text { Partial Agreement - look at } \\
\text { all aspects of life, acceptance } \\
\text { from staff, good therapeutic } \\
\text { relationships }\end{array}$ & $\begin{array}{l}\text { Partial Agreement - Physical } \\
\text { health did not improve, } \\
\text { reduction in trust of others, } \\
\text { service-users did value one } \\
\text { to ones with staff, staff did } \\
\text { not understand how service- } \\
\text { users mental illness affected } \\
\text { them. }\end{array}$ \\
\hline $\begin{array}{l}\text { Staffing levels will } \\
\text { mean that service- } \\
\text { users will be able to } \\
\text { access staff. }\end{array}$ & $\begin{array}{l}\text { Disagreement - staff } \\
\text { accessible all the time, } \\
\text { monitoring service-users. }\end{array}$ & $\begin{array}{l}\text { Disagreement - servicer-user } \\
\text { loneliness }\end{array}$ \\
\hline $\begin{array}{l}\text { Peer Mentors are a } \\
\text { source of hope, can } \\
\text { bridge the gap } \\
\text { between } \\
\text { professionals and } \\
\text { service-users and they } \\
\text { can use their } \\
\text { knowledge to support } \\
\text { service-users in ways }\end{array}$ & $\begin{array}{l}\text { Disagreement - peer } \\
\text { mentors brought knowledge } \\
\text { of mental illness from their } \\
\text { lived experience, hope and } \\
\text { inspired by peer mentors, } \\
\text { trust peer mentors. }\end{array}$ & $\begin{array}{l}\text { Disagreement - staff did not } \\
\text { understand how mental } \\
\text { illness affected service-users, } \\
\text { reduction in hope and trust } \\
\text { over time. }\end{array}$ \\
\hline
\end{tabular}


that professionals cannot. 
Appendix W - Ethical Approval - Swansea University

$29 / 04 / 2020$

Emall - PRITCHARD A. (967181) - Outlook

Ethics application Amy Pritchard

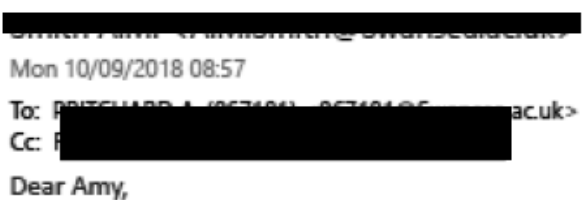

Application 010818a

Formal approval for this aspect of your study is now granted. I wish you well with your research.

Best wishes,

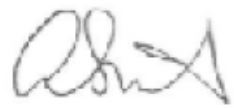

Angela Smith

Lecturer in Health Care Law and Ethics | Darlithydd mewn cyfraith Gofal lechyd a Moeseg

Chair, CHHS Ethics Committee

wwwswanseanacuk/humanandhealthsciences/research/research-ethics-committee

College of Human and Health Sciences | Coleg y Gwyddorau Dynol ac lechyd

Singleton Park | Parc Singleton

Swansea | Abertawe

Wales | Cymru

SA2 BPP

Room 700 Vivian Tower| Ystafell 700 , Vivian

Swansea University | Prifysgol Abertawe

Phone | Ffôn Ext. 01792602265

Email | Ebost a.m.smith@swansea.acuk

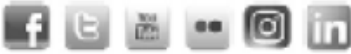

wow.swansea_ac.ulk I woww.abertawe,aculs

wowwswansea_aculk I wowwabertawe_aculk 


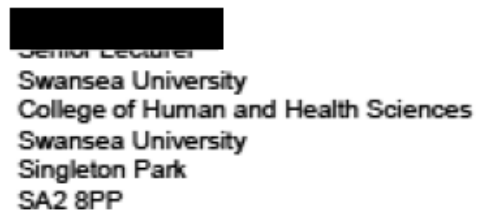

Study title:

A Realist Evaluation of Gellinudd Recovery Centre, Acceptance and Commitment Therapy and Physical Health

REC reference:

Protocol number: Education.

IRAS project ID:

18/WA/0315

RIO 011-18

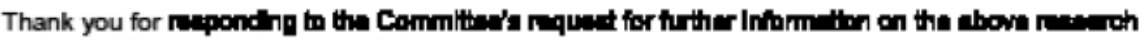
and submitting revised documentation.

The further information has been considered on behalf of the Committee by the Chair.

We plan to publish your research summary wording for the above study on the HRA website, together with your contact details. Publication will be no earlier than three months from the date of this opinion letter. Should you wish to provide a substitute contact point, require further information, or wish to make a request to postpone publication, please contact hra.studvreaistration.minhs.net outlining the reasons for your request.

Confirmation of ethical opinion

On behalf of the Committee, I am pleased to confirm a favourable ethical opinion for the above research on the basis described in the application form, protocol and supporting documentation as revised, subject to the conditions specified below.

Conditions of the favourable opinion

The REC favourable opinion is subject to the following conditions being met prior to the start of the study.

\section{Management nermission must be obtained from each host organisation orior to the start of the study} at the site concerned.

Management permission should be sought from all NHS organisations involved in the study in accordance with NHS research govemance arrangements. Each NHS organisation must confirm through the signing of agreements and/or other documents that it has given permission for the research to proceed (except where explicitly specified otherwise). 
Guidance on applying for HRA and HCRW Approval (England and Wales)/ NHS permission for research is available in the Integrated Research Application System, at www.hra.nhs.uk or at htto://umweroffonum.nhs,uk.

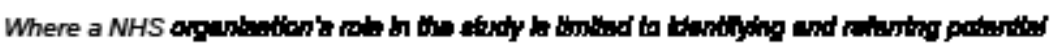

participants to research sites ("participant identification centre"), guidance should be sought from the $R \& D$ office on the information it requires to give permission for this activity.

For non-NHS sites, site management permission should be obtained in accordance with the procedures of the relevant host organisation.

Sponsors are not required to notify the Committee of management permissions from host organisations

\section{Reqistration of Clinical Trials}

All clinical trials (defined as the first four categories on the IRAS filter page) must be registered on a publically accessible database within 6 weeks of recruitment of the first participant (for medical device studies, within the timeline determined by the current registration and publication trees).

There is no requirement to separately notify the REC but you should do so at the earliest opportunity e.g. when submitting an amendment. We will audit the registration details as part of the annual progress reporting process.

To ensure transparency in research, we strongly recommend that all research is registered but for non-clinical trials this is not currently mandatory.

If a sponsor wishes to request a deferral for study registration within the required timeframe, they should contact hra.studvregistration inhs, net. The expectation is that all clinical trials will be registered, however, in exceptional circumstances non registration may be permissible with prior agreement from the HRA. Guidance on where to register is provided on the HRA website.

It is the responsibility of the sponsor to ensure that all the conditions are complied with before the start of the study or its initiation at a particular site (as applicable).

Ethical review of research sites

Non-NHS sites

\section{Approved documents}

The final list of documents reviewed and approved by the Committee is as follows:

\begin{tabular}{|c|c|c|}
\hline Document & Version & Date \\
\hline Copies of advertisement materials for research participants [Poster] & 1 & 16 July 2018 \\
\hline Covering letter on headed paper [Letter of Permission from Hafal] & 1 & 16 April 2018 \\
\hline $\begin{array}{l}\text { Evidence of Sponsor insurance or indemnity (non NHS Sponsors } \\
\text { only) [Letter to Evidence Sponsor Insurance or Indemnity] }\end{array}$ & 2 & 27 September 2018 \\
\hline $\begin{array}{l}\text { Interview schedules or topic guides for participants [Topic Guide for } \\
\text { Client and Carer Interviews] }\end{array}$ & 1 & 30 July 2018 \\
\hline IRAS Checklist XML [Checklist_07082018] & & 07 August 2018 \\
\hline IRAS Checklist XML [Checklist_01102018] & & 01 October 2018 \\
\hline $\begin{array}{l}\text { Letter from sponsor [Letter from Sponsor with Dr Sherrill } \\
\text { Snelgrove's details on] }\end{array}$ & 2 & 27 September 2018 \\
\hline Letters of invitation to participant [Invitation Letter for Clients] & 1 & 30 July 2018 \\
\hline Letters of invitation to participant [Invitation Letter for Carers] & 2 & 27 September 2018 \\
\hline Other [GCP Certificate] & 1 & 04 July 2018 \\
\hline Other [GDPR Certificate] & 1 & 27 June 2018 \\
\hline Other [Safeguarding Certificate] & 1 & 20 November 2017 \\
\hline
\end{tabular}

U: Lawmary FTHIC S CORRESPONDENCE 2018 CORRESPONDENCE 250186 18WA0315 fruther information fav opinion 5-10-18.dor 


\begin{tabular}{|c|c|c|}
\hline Other [Curriculum Vitae] & 1 & 30 July 2018 \\
\hline Other [Response to ethics No Opinion letter] & 1 & 28 August 2018 \\
\hline Participant consent form [Carer Consent Form] & 2 & 27 September 2018 \\
\hline $\begin{array}{l}\text { Participant consent form [Consent Form for Nominating Carer - } \\
\text { separate to consent form as declining to nominate does not impact } \\
\text { client participation] }\end{array}$ & 1 & 27 September 2018 \\
\hline Participant consent form [Client consent form] & 3 & 27 September 2018 \\
\hline $\begin{array}{l}\text { Participant information sheet (PIS) [Carer Participant Information } \\
\text { Sheet] }\end{array}$ & 2 & 27 September 2018 \\
\hline Participant information sheet (PIS) [Carer Debrief Sheet] & 2 & 27 September 2018 \\
\hline $\begin{array}{l}\text { Participant information sheet (PIS) [Client Participant Information } \\
\text { Sheet] }\end{array}$ & 2 & 27 September 2018 \\
\hline Participant information sheet (PIS) [Client Debrief Sheet] & 2 & 27 September 2018 \\
\hline REC Application Form [REC_Form_01102018] & & 01 October 2018 \\
\hline $\begin{array}{l}\text { Referee's report or other scientific critique report [Internal Scientific } \\
\text { Critique] }\end{array}$ & 1 & 27 September 2018 \\
\hline Research protocol or project proposal [Protocol - Version 2] & 2 & 28 September 2018 \\
\hline Summary CV for Chief Investigator (Cl) [Curriculum Vitae] & 2 & 27 September 2018 \\
\hline Summary CV for student [Curriculum Vitae] & 1 & 30 July 2018 \\
\hline Summary CV for supervisor (student research) [Curriculum Vitae] & 1 & 30 July 2018 \\
\hline Summary CV for supervisor (student research) [Curriculum Vitae] & 1 & 07 August 2018 \\
\hline Validated questionnaire [AAQ-II Questionnaire] & 1 & 30 July 2018 \\
\hline Validated questionnaire [CORE-Outcome Measure] & 1 & 30 July 2018 \\
\hline Validated questionnaire [CAMS-R Outcome Measure] & 1 & 30 July 2018 \\
\hline $\begin{array}{l}\text { Validated questionnaire [Health Confidence Scale Outcome } \\
\text { Measure] }\end{array}$ & 1 & 30 July 2018 \\
\hline $\begin{array}{l}\text { Validated questionnaire [Recovering Quality of Life Outcome } \\
\text { Measures] }\end{array}$ & 1 & 30 July 2018 \\
\hline $\begin{array}{l}\text { Validated questionnaire [Valued Living Questionnaire Outcome } \\
\text { Measure] }\end{array}$ & 1 & 30 July 2018 \\
\hline $\begin{array}{l}\text { Validated questionnaire [Views on Inpatient Care Outcome } \\
\text { Measure] }\end{array}$ & 1 & 30 July 2018 \\
\hline
\end{tabular}

Statement of compliance

The Committee is constituted in accordance with the Governance Arrangements for Research Ethics Committees and complies fully with the Standard Operating Procedures for Research Ethics Committees in the UK.

\section{After ethical review}

\section{Reporting requirements}

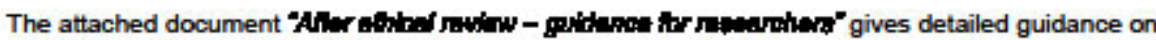
reporting requirements for studies with a favourable opinion, including:

- Notifying substantial amendments

- Adding new sites and investigators

- Notification of serious breaches of the protocol

- Progress and safety reports

- Notifying the end of the study

The HRA website also provides guidance on these topics, which is updated in the light of changes in reporting requirements or procedures. 


\section{User Feedback}

The Health Research Authority is continually striving to provide a high quality service to all applicants and sponsors. You are invited to give your view of the service you have received and the application procedure. If you wish to make your views known please use the feedback form available on the HRA website: http://www.hra.nhs.uk/about-the-hra/governance/quality-assurance/

\section{HRA Training}

We are pleased to welcome researchers and R\&D staff at our training days - see details at http://www.hra.nhs.uk/hra-trainingl

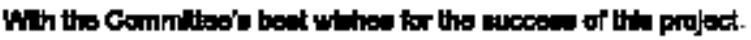

Yours sincerely

$$
\text { The Exy }
$$

Dr M J Lawrence

Chair

Email:penny.beresford@wales.nhs.uk

Enclosures:

"After ethical review - guidance for nowishers' [SL-AR2]

Copy to:

Dr Sherrill Snelgrove, Swansea University 


\section{Appendix Y - Ethical Amendment Approval - Commissioners}

Ymchwil lechyd a Gofal Cymru

Health and Care

Research Wales
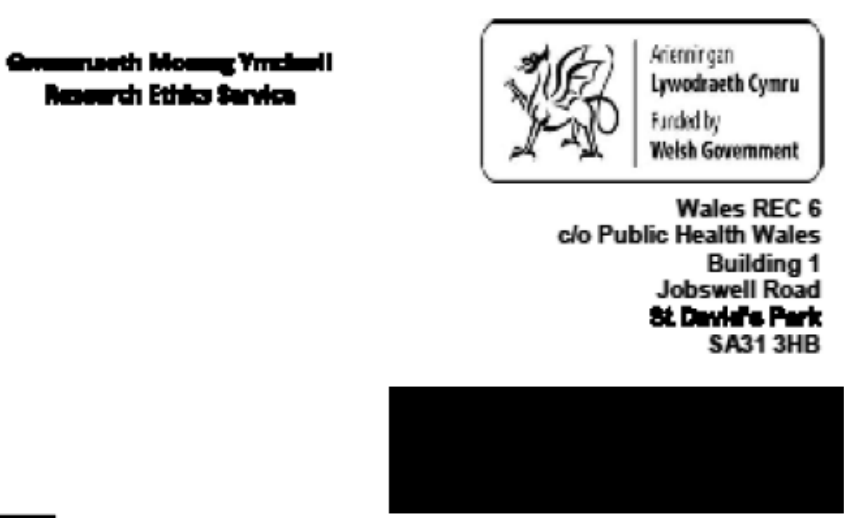

Please note: This is the favourable

epinion of the REC only and does not

allow the amendment to be

implemented at NHS sites in England

until the outcome of the HRA

assessment has been confirmed.

07 August 2019

Miss Amy Pritchard

PhD Student

Swansea University

Swansea University

Singleton Campus, Singleton Park

Vivian Tower 3rd Floor Room 318

SA2 8PP

Dear Miss Pritchard

Study title:

A Realist Evaluation of Gellinudd Recovery Centre, Acceptance and Commitment Therapy and Physical Health Education.

REC reference: 18/WA/0315

Protocol number:

Amendment number:

Amendment date: RIO 011-18

IRAS project ID:

22 July 2019

250186

The above amendment was reviewed at the meeting of the Sub-Committee held on 29 July 2019 by the Sub-Committee in correspondence.

Ethical opinion

The members of the Committee taking part in the review gave a favourable ethical opinion of the amendment on the basis described in the notice of amendment form and supporting documentation.

Approved documents 
The documents reviewed and approved at the meeting were:

\begin{tabular}{|l|l|l|}
\hline Document & Version & Date \\
\hline $\begin{array}{l}\text { Interview schedules or topic guides for participants [Commisioners } \\
\text { Interview Topic Guide] }\end{array}$ & 1 & 19 July 2019 \\
\hline Non-validated questionnaire [Commissioners] & 1 & 19 July 2019 \\
\hline $\begin{array}{l}\text { Notice of Substantial Amendment (non-CTIMP) [Amendment } \\
\text { Number 1, 19 Jul 2019] }\end{array}$ & & 22 July 2019 \\
\hline Other [Commissioners - Notes on Amendments to Protoco[] & 1 & 19 July 2019 \\
\hline Other [Commissioning/Referrals Debrief Sheet] & 1 & 19 July 2019 \\
\hline Other [Organisation Information Document] & 1.0 & 01 August 2019 \\
\hline Other [Schedule of Events] & 1.0 & 01 August 2019 \\
\hline Participant consent form & 1 & 19 July 2019 \\
\hline $\begin{array}{l}\text { Participant information sheet (PIS) [Commissioner Participant } \\
\text { Information Sheet ABMU] }\end{array}$ & 1 & 19 July 2019 \\
\hline $\begin{array}{l}\text { Participant information sheet (PIS) [Commissioner Participant } \\
\text { Information Sheet Hywel Dda] }\end{array}$ & 1 & 19 July 2019 \\
\hline Participant information sheet (PIS) [Swansea Bay] & 2.0 & 31 July 2019 \\
\hline Participant information sheet (PIS) [Hwyel Dda] & 2.0 & 31 July 2019 \\
\hline Research protocol or project proposal & 3.0 & 19 July 2019 \\
\hline
\end{tabular}

Membership of the Committee

The members of the Committee who took part in the review are listed on the attached sheet.

\section{Working with NHS Care Organisations}

Sponsors should ensure that they notify the R\&D office for the relevant NHS care organisation of this amendment in line with the terms detailed in the categorisation email issued by the lead nation for the study.

\section{Statement of compliance}

The Committee is constituted in accordance with the Governance Arrangements for Research Ethics Committees and complies fully with the Standard Operating Procedures for Research Ethics Committees in the UK.

\section{HRA Learning}

We are pleased to welcome researchers and research staff to our HRA Learning Events and online learning opportunities- see details at: https://www.hra.nhs.uk/planning-and-improvingresearch/learning/

18/WA0315: $\quad$ Please quote this number on all correspondence

Yours sincerely

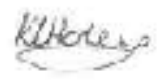




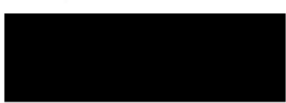

Swansea University

College of Human and Health Sciences

Swansea University

Singleton Park

SA2 8PP

07 August 2019

Dear Dr Rance

\section{HRA and Health and Care}

Research Wales (HCRW)

Approval Letter

Study title:

A Realist Evaluation of Gellinudd Recovery Centre, Acceptance and Commitment Therapy and Physical Health Education.

IRAS project ID: 250186

Protocol number:

RIO 011-18

REC reference:

18/WA/0315

Sponsor

Swansea University

I am pleased to confirm that HRA and Health and Care Research Wales (HCRW) Anoroval has been given for the above referenced study, on the basis described in the application form, protocol, supporting documentation and any clarifications received. You should not expect to receive anything further relating to this application.

Please now work with participating NHS organisations to confirm capacity and capability, in

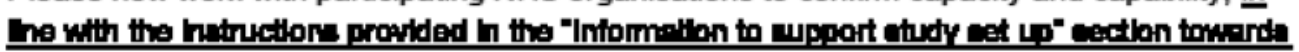
the end of this letter.

How should I work with participating NHS/HSC organisations in Northern Ireland and Scotland?

HRA and HCRW Approval does not apply to NHS/HSC organisations within Northern Ireland and Scotland.

If you indicated in your IRAS form that you do have participating organisations in either of these devolved administrations, the final document set and the study wide governance report 
(including this letter) have been sent to the coordinating centre of each participating nation. The relevant national coordinating function/s will contact you as appropriate.

Please see IRAS Help for information on working with NHS/HSC organisations in Northern Ireland and Scotland.

How should I work with participating non-NHS organisations? HRA and HCRW Approval does not apply to non-NHS organisations. You should work with your non-NHS organisations to obtain local aqreement in accordance with their procedures.

What are my notification responsibilities during the study?

The doumment "After Ethical Review - guidance for sponsors and investigators", baud with your REC favourable opinion, gives detailed guidance on reporting expectations for studies, including:

- Registration of research

- Notifying amendments

- Notifying the end of the study

The HRA website also provides guidance on these topics, and is updated in the light of changes in reporting expectations or procedures.

Who should I contact for further information?

Please do not hesitate to contact me for assistance with this application. My contact details are below.

Your IRAS project ID is 250186. Please quote this on all correspondence.

Yours sincerely,

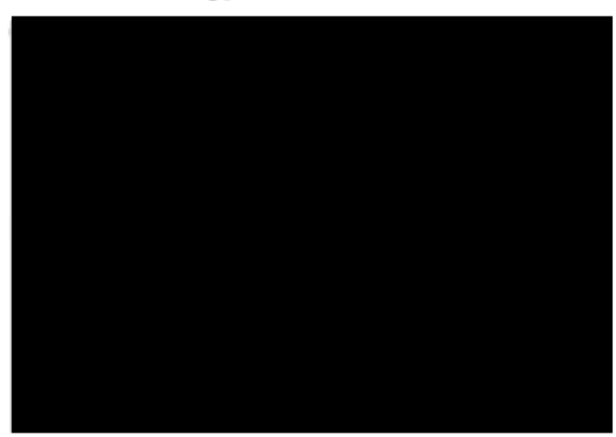




\section{Appendix Z - Commissioner Participant Information Sheet}

\section{Student Study: A Realist Evaluation of Woodlands}

We would like to invite you to take part in our research study. Joining the study is entirely up to you, before you decide we would like you to understand why the research is being done and what it would involve for you. One of our team will go through this information sheet with you, to help you decide whether or not you would like to take part and answer any questions you may have. We'd suggest this should take about 10 minutes. Please feel free to talk to others about the study if you wish. The first part of the Participant Information Sheet tells you the purpose of the study and what will happen to you if you take part. Then we give you more detailed information about the conduct of the study.

Do ask if anything is unclear.

\section{Summary}

The aim of this interview is to provide an independent perspective on the current referrals process at Woodlands. This is important as previous interview data has suggested that there have been difficulties with the current referrals process and therefore, we think it would be beneficial to understand this from a perspective that is external to the service. It is important to gain this perspective so the research team can gain a more balanced understanding of the current referrals process and situation specifically relating to Woodlands.

Participants will be required to participate in a 30-45-minute telephone interview. The interview will give participants the opportunity to share their experience of working with Woodlands, explore their understanding and opinion of the service and to discuss the current process of referrals and any issues that have arisen. Participants who are eligible are those who have been involved in the referrals process to Woodlands, this can include both successful and unsuccessful referrals.

\section{1) Explanation:}

The purpose of this research is to provide additional information to support the current, independent evaluation that is being undertaken at Woodlands. A consistent theme that has arisen from the current interview data is difficulties with the referrals process. Therefore, the purpose of this research is to explore a different perspective of other individuals involved in the referrals process. It is anticipated that there will be 3-5 interviews conducted with commissioners who have been involved in the referrals process at Woodlands in order to understand a new, and potential different narrative around factors that have impacted upon referrals to Woodlands.

You have therefore been nominated as you are involved in the referrals process at Woodlands and will be able to provide valuable information to enable the researcher to better understand the current referrals process and how this has impacted upon the client numbers at Woodlands. 
We are also interested to hear your experience of working with and referring clients to Woodlands.

\section{a) What would taking part involve?}

You will be invited to take part in a telephone interview with the researcher, which will last between 30-45 minutes. All the interviews will be audio recorded, but the audio tapes will be destroyed once they have been typed up by the researcher. You will not be asked to disclose any personal information; however, you will be required to share your personal experiences of working with Woodlands within your job role.

\section{b) What are the possible benefits of taking part?}

You will have the opportunity to:

- share your understanding of what Woodlands provides clients to support them in their mental health recovery;

- $\quad$ provide your opinion on the current referrals process;

- identify any factors that may have facilitators or barriers in the referrals process at Woodlands;

- have your voice heard in relation to the process of referrals at Woodlands so a balanced account can be presented, and the research team can draw conclusions from several different perspectives;

- contribute to the current independent evaluation of Woodlands.

c) What are the possible disadvantages and risks of taking part?

- The possible risks are minimal, however if you have experienced any difficulties in your working relationship then this could cause you to feel upset or distressed, if this arises the researcher will support you and you are able to stop the interview at any point.

\section{2) Supporting Information}

\section{a) What if something goes wrong?}

- If you have any specifically issues relating to this project and would like to contact the research team, then please contact the Chief Investigator on j.y.rance@swansea.ac.uk

- There is also additional information below with regards to the complaints process.

b) What will happen if I don't want to carry on with the study?

- As participation is entirely voluntary you can change your mind at a later stage, this will be at the end of the data collection period the $31^{\text {st }}$ December 2019. If you withdraw your consent before this date, your data will be removed and destroyed. If you withdraw after this date, the interview data will already have been analysed and incorporated within the write up of the findings and therefore withdrawing data will not be possible;

- To withdraw your consent please email the researcher directly and this will be done immediately. 


\section{c) Will my information be kept confidential?}

- Swansea University is the sponsor for this study based in the United Kingdom. We will be using information from your interview in order to undertake this study and will act as the data controller for this study. This means that we are responsible for looking after your information and using it properly.

- Your rights to access, change or move your information are limited, as we need to manage your information in specific ways for the research to be reliable and accurate.

- If you withdraw from the study, we will keep the information about you that we have already obtained. To safeguard your rights, we will use the minimum personally identifiable information possible.

- You can find out more about how we use your information researchgoverance@swansea.ac.uk.

- Only Amy Pritchard will have access to the audio recorders, and Jaynie Rance and Michael Coffey will only have access once the data is anonymised. All data, including electronic consent forms and interview transcripts will be stored as password protected files on a password protected computer based at Swansea University.

- All data used within publications will use pseudonyms.

\section{d) What will happen to the results of this study?}

- Results will be presented at conferences, written up for journal articles and will be part of the write up of the key researcher's PhD thesis. If any of your individual data is presented and/or published, the data will be totally anonymous, therefore there will be no means of identifying you as the individual involved.

\section{e) Who is organising and funding this study?}

- Swansea University is organising this study. The study is part funded by Swansea University and Hafal, although this is an independent evaluation and therefore Hafal are not involved in the research project or contributing to the collection of data.

\section{f) Who has reviewed this study?}

- All research in the NHS is looked at by an independent group of people, called a Research Ethics Committee, to protect your interests. This study has been reviewed and given favourable opinion by Swansea Research Ethics Committee 6.

\section{g) Who to complain to for Data/Management/Health issues?}

The data controller for this project will be Swansea University. The University Data Protection Officer provides oversight of university activities involving the processing of personal data, and can be contacted at the Vice Chancellors Office:

dataprotection@swansea.ac.uk

Your personal data will be processed for the purposes outlined in this information sheet. 
Standard ethical procedures will involve you providing your consent to participate in this study by completing the consent form that has been provided to you. However, the legal basis on which this task is being performed is public interest, approved by the departmental Research Ethics Committee.

Details of your individual rights are available on the ICO website at:

https://ico.org.uk/for-organisations/data-protection-reform/overview-of-the-

gdpr/individuals-rights/

\section{h) Further information and contact details}

- Alternatively, if you would like to discuss this with an independent contact who is part of your health board please use the following details:

[insert local health board information relevant to each commissioner participant] 
Appendix AA - Commissioner Consent Form

Commissioners Participant Consent Form - A Realist Evaluation of Woodlands

Chief Investigator - Jaynie Rance

\begin{tabular}{|c|c|}
\hline & Participant Initial \\
\hline $\begin{array}{l}\text { 1. I (the participant) confirm that I have read and understand } \\
\text { the information sheet for the above study which is } \\
\text { attached to this form. }\end{array}$ & \\
\hline $\begin{array}{l}\text { 2. I understand that my participation is voluntary and that I } \\
\text { am free to withdraw at any time, without giving any } \\
\text { reasons. }\end{array}$ & \\
\hline $\begin{array}{l}\text { 3. I understand what my role will be in this research, and all } \\
\text { my questions have been answered to my satisfaction. }\end{array}$ & \\
\hline $\begin{array}{l}\text { 4. I understand that I am free to ask any questions at any } \\
\text { time before and during the study. }\end{array}$ & \\
\hline $\begin{array}{l}\text { 5. I have been informed that the information I provide will be } \\
\text { safeguarded. }\end{array}$ & \\
\hline $\begin{array}{l}\text { 6. I am happy for the information I provide to be } \\
\text { pseudonymised in academic papers and other formal } \\
\text { research outputs. }\end{array}$ & \\
\hline 7. I am willing for my information to be audio recorded. & \\
\hline $\begin{array}{l}\text { 8. I have been provided with a copy of the Commissioner } \\
\text { Participant Information Sheet. }\end{array}$ & \\
\hline $\begin{array}{l}\text { 9. I understand that my research data will be securely stored } \\
\text { for } 10 \text { years and will then be destroyed. }\end{array}$ & \\
\hline
\end{tabular}

Thank you for your participation in this study. Your help is very much appreciated.

Print name of participant

Signature

Date

Print name of researcher

Signature

Date

This study is being conducted by Swansea University, College of Human and Health Science.

When complete: Original copy for participant, one copy to be retained by researcher 
Appendix BB - Commissioner Debrief Sheet

\section{A Realist Evaluation of Woodlands - Commissioner Debrief Sheet}

Thank you for taking part in the interview today. Your time and effort throughout this research project has been hugely appreciated.

If you have any queries about this study, please do not hesitate to contact Amy using the email address provided below. As all data collection is anonymous if you would like your data to be removed from the study you will need to provide Amy with your pseudonym (fake name) you chose for the interview.

Chief Investigator - Jaynie Rance - Email Address 
Appendix CC - Commissioner Topic Guide

\section{Commissioner Interview Topic Guide}

Introduction:

Thank the participant for agreeing to the interview. Check to ensure the participant is aware of the purpose of the research, has given informed consent and is aware and OK with the interview being recorded. Ask them if they have any questions before the interview begins.

Explain that you are here to talk to them because of their role in the referrals process at Woodlands.

Topics to be covered during the interview:

\section{Understanding of Woodlands:}

These questions will relate to the commissioners understanding of what Woodlands provides as a service and to gain an understanding of how this differs to other providers. These questions will also relate to commissioners understanding of who is suitable for Woodlands.

For example:

1. What is your understanding of what Woodlands provides a client in terms of their mental health recovery?

2. What is your understanding about who is suitable for Woodlands?

\section{Experiences of working with Woodlands:}

These questions will relate to the commissioner's experience of working with Woodlands during the referrals process. This will relate to both successful and unsuccessful referrals and will try to explore why these have, or have not been successful. This will also relate to their awareness of how clients have responded to Woodlands, and if any clients have requested to leave the service after admission.

For example:

1. What factors lead to this being an unsuccessful experience?

2. What have the experiences of clients you have referred to Woodlands been?

\section{Gaining an understanding of the decision-making process for referrals:}

These questions will relate to how decisions are made with regards to client referrals and begin to understand what Woodlands fulfils, and does not fulfil, when commissioners make decisions about client placements. The questions will also relate to whether commissioners believe there is a current issue in terms of referrals to Woodlands:

For example: 
1. Do you believe there is an issue in terms of referrals to Woodlands? If so, what factors do you think are contributing to this issue?

2. I have been informed by the service that as of April 2019, Woodlands reduced its price are the cheapest provider for females and the second cheapest for males - can you explain what other factors, beside finances, are considered when making decisions about appropriate placements for clients?

\section{Ending the Interview:}

- Is there anything else you'd like to say?

Close the interview and thank the participant for their participation. 
Appendix DD - Commissioner Initial Codes

\begin{tabular}{|c|c|}
\hline Code Name & Description \\
\hline $\begin{array}{l}\text { Access to Psychological } \\
\text { Therapies }\end{array}$ & $\begin{array}{l}\text { Decision-making based upon the accessibility of psychological } \\
\text { therapies at the service. Commissioners had an awareness of } \\
\text { what psychological therapies services provide and used this } \\
\text { information if a specialist placement was required. }\end{array}$ \\
\hline $\begin{array}{l}\text { Appropriateness of } \\
\text { Placement }\end{array}$ & $\begin{array}{l}\text { Consideration given to the appropriateness of a placement for } \\
\text { service-users and how this feeds into the decision-making about } \\
\text { a service-user's placement. }\end{array}$ \\
\hline Awareness of Service & $\begin{array}{l}\text { How the commissioner became aware of the service and what } \\
\text { understanding they had about what the service provides. }\end{array}$ \\
\hline C CAP System & $\begin{array}{l}\text { The framework used to identity placements for service-users and } \\
\text { how this is utilised within decision-making. }\end{array}$ \\
\hline $\begin{array}{l}\text { Service-user Group for } \\
\text { Locked Rehab }\end{array}$ & $\begin{array}{l}\text { The service-user group commissioners considered suitable for } \\
\text { locked rehabilitation or considers the current need and demand } \\
\text { of locked rehabilitation within the current climate. }\end{array}$ \\
\hline $\begin{array}{l}\text { Service-users for the } \\
\text { Service }\end{array}$ & $\begin{array}{l}\text { The service-user group the commissioners determine as suitable } \\
\text { for the service based upon their experience of working with the } \\
\text { service. }\end{array}$ \\
\hline $\begin{array}{l}\text { Compared to Other } \\
\text { Providers }\end{array}$ & $\begin{array}{l}\text { Consideration of whether there are any differences between the } \\
\text { service being evaluated and other providers and how this might } \\
\text { be influencing referrals. }\end{array}$ \\
\hline Decision-Making Factors & $\begin{array}{l}\text { Any factor that influences how commissioners make decisions } \\
\text { about service-user placements, which may be impacted upon the } \\
\text { number of referrals received at the service. }\end{array}$ \\
\hline Duration of Care & $\begin{array}{l}\text { Considers whether referring service-users to a new service may } \\
\text { lengthen service-users stay within services. }\end{array}$ \\
\hline Proximity to family & $\begin{array}{l}\text { Consideration of whether the service is accessible for the } \\
\text { service-user's family and how this influences decision-making } \\
\text { with a prioritisation of service-user placements being within the } \\
\text { health board locality. }\end{array}$ \\
\hline Least Restrictive & $\begin{array}{l}\text { Consideration of how commissioner's decision-making is guided } \\
\text { by ensuring the least restrictive option is selected for service- } \\
\text { users, with a shift towards open rehabilitation and community } \\
\text { placements compared to secure services. }\end{array}$ \\
\hline Locality & $\begin{array}{l}\text { Considers the location of the service which is prioritised in } \\
\text { commissioner's decision-making. }\end{array}$ \\
\hline Management of Risk & $\begin{array}{l}\text { Considers the level of risk the service tolerates in comparison to } \\
\text { other locked rehabilitation services. The service's ability or } \\
\text { willingness to manage service-user's risk (self-harm, suicide or } \\
\text { aggression and violence) and how this linked with declining } \\
\text { referrals to the service. }\end{array}$ \\
\hline Meet Service-user Needs & $\begin{array}{l}\text { How commissioner's decision-making is influenced by whether } \\
\text { the service is able to meet service-user's needs. }\end{array}$ \\
\hline Need for Consequences & $\begin{array}{l}\text { Consideration of whether the service's approach to structure and } \\
\text { consequences is suitable for all service-users at the service and } \\
\text { how this has contributed to a breakdown in service-user } \\
\text { placements. }\end{array}$ \\
\hline Need for Locked Rehab & $\begin{array}{l}\text { Discussion about whether there is a current need for locked } \\
\text { rehabilitation within the current climate. }\end{array}$ \\
\hline
\end{tabular}




\begin{tabular}{|l|l|}
\hline $\begin{array}{l}\text { Not an Issue with } \\
\text { Referrals }\end{array}$ & $\begin{array}{l}\text { Determines whether commissioner align that the service has } \\
\text { received low referrals. }\end{array}$ \\
\hline Personality Disorder & $\begin{array}{l}\text { Discusses about how a diagnosis of personality disorder is } \\
\text { typically associated with requiring locked rehabilitation. }\end{array}$ \\
\hline Quality Rating & $\begin{array}{l}\text { Considers how the framework utilises a quality rating and how } \\
\text { this influences commissioner's decision-making. }\end{array}$ \\
\hline Test Open Door & $\begin{array}{l}\text { Commissioners emphasise the importance of service-users being } \\
\text { tested with an open door, which cannot be achieved within } \\
\text { locked rehabilitation services. }\end{array}$ \\
\hline Uniqueness of Service & $\begin{array}{l}\text { Determines whether commissioners perceive the service as } \\
\text { providing something different compared to other services. }\end{array}$ \\
\hline and Placements & $\begin{array}{l}\text { Provides examples of how the service has declined referrals to } \\
\text { the service, the rationale provided for this decision and why } \\
\text { placements have broken down or been unsuccessful for service- } \\
\text { users. }\end{array}$ \\
\hline $\begin{array}{l}\text { Use NHS Provided } \\
\text { Services }\end{array}$ & $\begin{array}{l}\text { Considers how health boards prioritise placing service-users } \\
\text { within NHS provided services before considering other providers. } \\
\text { Commissioners identify what services they provide within their } \\
\text { health board and why this may have influenced why service- } \\
\text { users are not being referred to the service. }\end{array}$ \\
\hline
\end{tabular}


Appendix EE - Commissioner Refined Codes

\begin{tabular}{|c|c|c|}
\hline Category & Description & Initial Codes included within Category \\
\hline $\begin{array}{l}\text { Decision- } \\
\text { Making Factors }\end{array}$ & $\begin{array}{l}\text { Factors that influence } \\
\text { commissioner's } \\
\text { decision-making and } \\
\text { how these have } \\
\text { influenced referrals to } \\
\text { the service. }\end{array}$ & 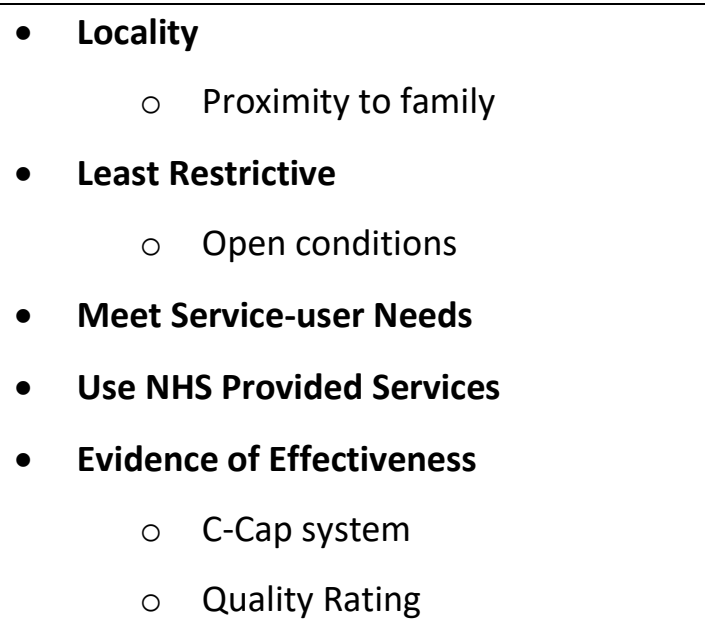 \\
\hline $\begin{array}{l}\text { Understanding } \\
\text { of the Service }\end{array}$ & $\begin{array}{l}\text { Commissioner's } \\
\text { understanding of the } \\
\text { service and how this } \\
\text { differs from what other } \\
\text { services provide and } \\
\text { how this might have } \\
\text { influenced referrals to } \\
\text { the service. }\end{array}$ & $\begin{array}{l}\text { - } \text { Awareness of the Service } \\
\text { - Compared to Other Providers } \\
\text { - } \quad \text { Not an Issue with Referrals } \\
\text { - Uniqueness of the Service }\end{array}$ \\
\hline $\begin{array}{l}\text { Current Market } \\
\text { Demand - Need } \\
\text { and the Use of } \\
\text { Locked } \\
\text { Rehabilitation } \\
\text { Services }\end{array}$ & $\begin{array}{l}\text { The current use and } \\
\text { need for locked } \\
\text { rehabilitation for } \\
\text { service-users within } \\
\text { Wales and how this } \\
\text { might have influenced } \\
\text { referrals to the service. } \\
\text { Commissioners classified } \\
\text { Woodlands as a locked } \\
\text { rehabilitation service. }\end{array}$ & 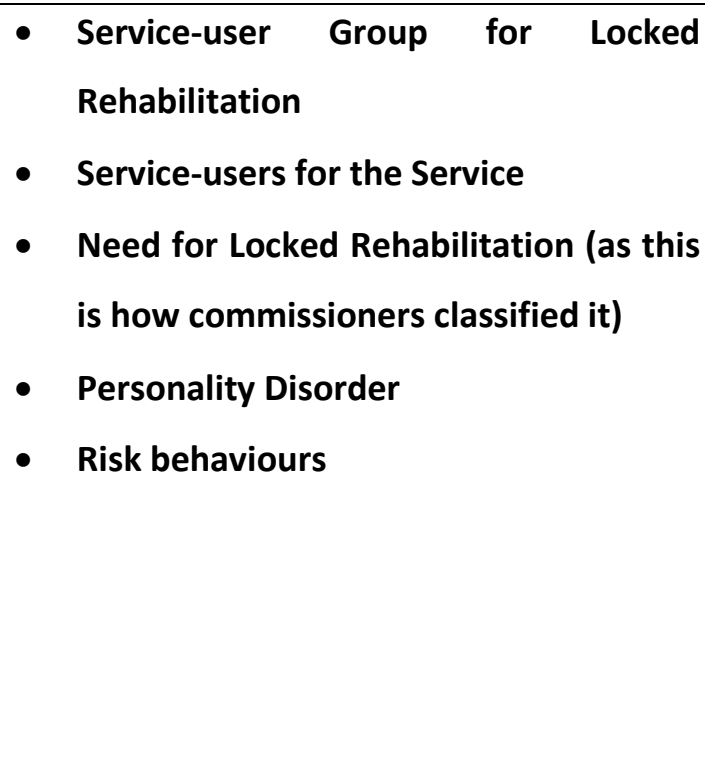 \\
\hline $\begin{array}{l}\text { Unsuccessful } \\
\text { Referrals and } \\
\text { Placements }\end{array}$ & $\begin{array}{l}\text { Reasons provided that } \\
\text { commissioners felt } \\
\text { contributed to } \\
\text { unsuccessful referrals to }\end{array}$ & $\begin{array}{l}\text { - } \quad \text { Management of Risk } \\
\text { - } \quad \text { Need for Consequences. }\end{array}$ \\
\hline
\end{tabular}




\begin{tabular}{|l|l|}
\hline the service and the \\
breakdown in service- \\
user's placements at the \\
service.
\end{tabular} \mid

Please note that some initial codes were not included within the refined codes as these were not discussed across participants, or the was insufficient prevalence within the data extracts to consider it part of an overarching category. These initial codes often were only in the data once. 

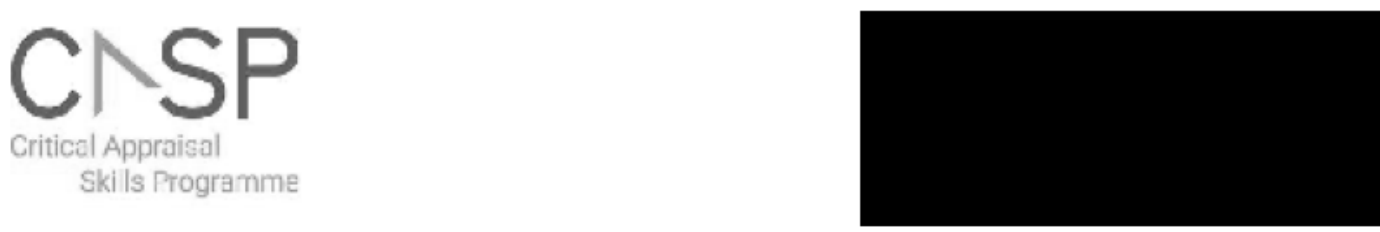

CASP Chectlist 10 questions to help you make sense of a Qualitotve resewch

How to use thk apprabal tapl: Three brow /ssues need to be conskdered when appraldng a qualitative study:

Are the results of the study welid? (Section A]
What are the results?
will the results help locally?
[Section B]

The 10 questions on the folowing pages are deslgned to help you think about these ls5ues spstematicalhy. The first two questlons ane screening questlons and ban be answered quickdy. If the answer to both is "yose, it is worth proceeding with the remaining questions. There is some degree of overlap between the questions, you are asked to record a "yes". "no" or "can't tell" to most of the questions. A number of italkelsed prompts are given efter exch question. These are desimed to remind you why the question is important Record your reasons for your answers in the spaces provided.

About These checklists were desifned to be used as eductional pedapagic tools, as part of a workshop setting, therefore we do not suggett a scoring 5ystem. The core CA5P checkllsts (randamlsed controlled tral is spstematte reviem) were based an JAMA 'Users' guldes to the medleal Ilterature 1994 (adapted from Guyatt GH, Sackett DL, and Cook D), and plloted $w$ th health care practutoners.

For eath new chect|lst, a group of experts were essembled to dewelop and plkt the checkllst and the workshop format with which it would be used. Over the years overall adjustments have been made to the format, but a recent surver of checklst users relterated that the basic format continues ta be useful and appraprlate.

Referencing: we recommend using the Harvard style cltation, le: Critcol Aparaisa' Skiks

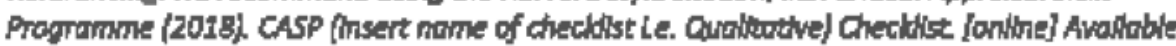
ot Uill Accessed: Dote Acressed.

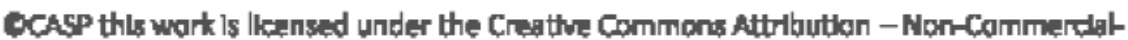

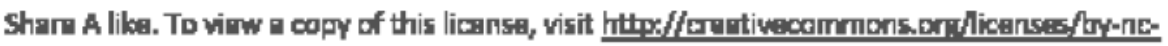

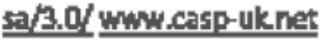


Paper for appralt=l and noloranca:

\section{Sacton A: Ane the realut velde?}

1. Was there a clatr

thimmint of tha alms of

the research?

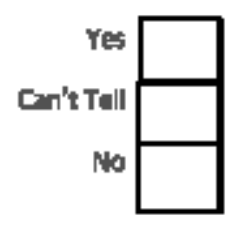

HINT: Consider

- what was the rool of the rusareh

- why it was thought impertant

- Ir riminas

Comments:

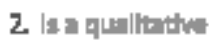
methodaloy uppropricte?

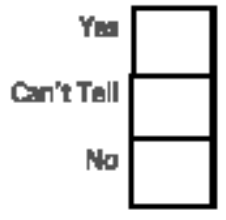

HINT:Considur

- If the rasearch secks to Interprat or Illum|nrte the actlona and/or gublecthe experlences of research particlpants

- Is quallirtive reseaneh the right methodology for addresdng the newarch gosal

Comments:

Is kwarth mantruln?

3. Was the research dedgn appropriate to

iddress the alriv of the reanurch?

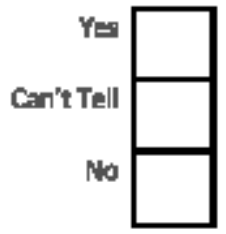

HINT: Consider

- if the researcher hes justified the

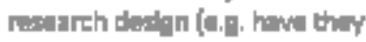
discossed how they decided which method tor un]

Comments: 


\section{CNSP \\ Critical Appraisal \\ Skills Programm}

4. Way the recrultment stritury appropilate tre the aims of the

resuran?

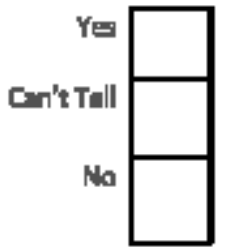

HINT: Corsilder

- If the researcher has explahed how the partd dpents ware sulectrod

- If they aplahed why the partidpanta they selected were the most

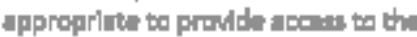
type of knowledge sought by the stuth - If there are any discussions around recruttment (e.t. why sorre people chesa not to bals part

Comments:

5. Was the data collected in a way that addreand the research isue?

$r$

HINT: Corisider

- If the Eatting for the data collectlon was Jwithind

- If it is clear how data were collected le,g. focise group, saml-structurad Intandaw etcil

- If the researcher has justifled the methods choren

- If the researcher has made the methods explicit fe\& for interview method, is there an indication of how intervicus are conducted, or did they use a topic buide!

- If methots were modified during the studk. If so, hos the regen the explained how and why

- If the farm of dito s derriect tape record/n F, video mutertal, notes etc

- If the rexeurcher has discissed miturtion of dot

Comments: 
6. Has the relationship borwoun reartherand perticipents been

adequitrely consldered?

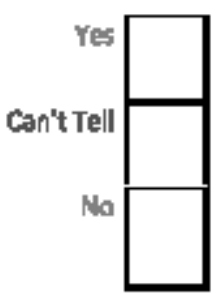

HINT: Consditr

- If tha researeher cttleally exinined their own role, potentlal blas and Influence during (i) formulation of the resarch quextans (b) date collection, including sample recrultement and cholos of

location

- How the researcher responded to Events durlng the study and whether they censsidered tha Implloritlons of uny dianges in the

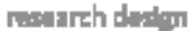

Comments:

Section Bi What are the results?

7. Have ethlcal ksues beer tulter lnto consideration?

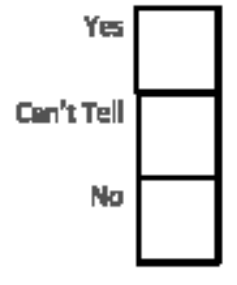

- If there are sufficient detils of how the rasearch wra moplalnad to purtdpants for the nesider to assess whether ethleal stond ords were molmtolned

- If the regracher has discused beves: ralced by the study leg. 1esues around informed consent or tonfifidentialliy or how they have handlad the affects of the stivty on the participants during and after the sodyl

- If approval has bean soungt fram the ethles commitites

Comments: 
a. Wan the data analyals suffidenty rhorate?

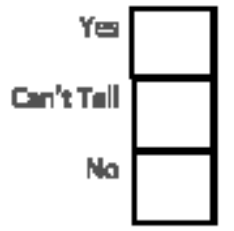

HiNT: Conside

- If there $b$ m in-depth dexpiption of the andyly procuss

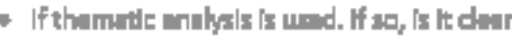
how the cotedoriest themes were defired

from the date

- Whathar tha ressarcher aplains how the data presented were selected from the ortginal gmple to demunstrite the andints prectes

- If esrifidart data are presented to support the finding:

- To wherit extent contradictory dutrare taken Inmo accoumt

- Whether tha rosoancher erttlcally eamined their own role, potential bias and influence during arrelyds and suluction of dita for presentation

Comment:

9. Is there a ceal statement of findln $\rightarrow$ ?

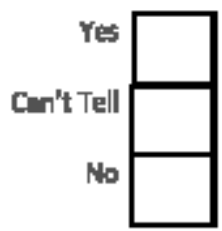

HINT: Consider whether

- If the firdinses ere enplicit

- If there ls adequate discussion of the evidence both for and andinst the resasncher's arguments

- If the researcher has dlsoussed the credbillty of thelr findiny (e. triangulatlen, respondent validathon, mere then one analyot

- If the finding are discussed in relation to the orlalmal manth quatidan

Comments: 


\section{CASP \\ Critical Appraisal \\ Skills Programme}

Section C: W|l the results hedp lacilly?

10. How voluablo lathe

research?
HINT: Con:ulder

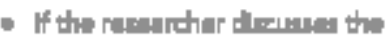
contribution the study males to existing lanawlody or undantanding la.e do they conelder the find ings In retatlan to current predice or pdlcy, or relovirit regench-

based literature

- If thoy ldantif new arases where researd

is necessary

- If the reseanchers hove discussed whether or haw tha findlins on ba transfarred to other populations or corbsidered other

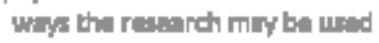

Comments: 


\section{Evaluation Tool for Quantitative Research Studies}

Building on work within a project exploring the feasibility of undertaking systematic reviews of research literature on effectiveness and outcomes in social care, a set of evaluation tools have been developed to assist in the critical appraisal of research studies. The evaluation tool for quantitative studies contains six sub-sections: study evaluative overview; study, setting and sample; ethics; group comparability and outcome measurement; policy and practice implications; and other comments. It provides a template of key questions to assist in the critical appraisal of quantitative research studies.

\begin{tabular}{|c|c|}
\hline Review Area & Key Questions \\
\hline \multicolumn{2}{|c|}{ (1) STUDY OVERVIEW } \\
\hline $\begin{array}{l}\text { Bibliographic } \\
\text { Details }\end{array}$ & 0. Author, title, source (publisher and place of publication), year \\
\hline Purpose & $\begin{array}{l}\text { 1. What are the aims of the study? } \\
\text { 2. If the paper is part of a wider study, what are its aims? }\end{array}$ \\
\hline Key Findings & 3. What are the key findings of the study? \\
\hline $\begin{array}{l}\text { Evaluative } \\
\text { Summary }\end{array}$ & $\begin{array}{l}\text { 4. What are the strengths and weaknesses of the study and theory, policy } \\
\text { and practice implications? }\end{array}$ \\
\hline \multicolumn{2}{|c|}{ (2) STUDY, SETTING, SAMPLE AND ETHICS } \\
\hline The Study & $\begin{array}{l}\text { 5. What type of study is this? } \\
\text { 6. What was the intervention? } \\
\text { 7. What was the comparison intervention? } \\
\text { 8. Is there sufficient detail given of the nature of the intervention and the } \\
\text { comparison intervention? } \\
\text { 9. What is the relationship of the study to the area of the topic review? }\end{array}$ \\
\hline Setting & 10. Within what geographical and care setting was the study carried out? \\
\hline Sample & $\begin{array}{l}\text { 11. What was the source population? } \\
\text { 12. What were the inclusion criteria? } \\
\text { 13. What were the exclusion criteria? } \\
\text { 14. How was the sample selected? } \\
\text { 15. If more than one group of subjects, how many groups were there, and } \\
\text { how many people were in each group? } \\
\text { 16. How were subjects allocated to the groups? } \\
\text { 17. What was the size of the study sample, and of any separate groups? } \\
\text { 18. Is the achieved sample size sufficient for the study aims and to } \\
\text { warrant the conclusions drawn? } \\
\text { 19. Is information provided on loss to follow up? } \\
\text { 20. Is the sample appropriate to the aims of the study? } \\
\text { 21. What are the key sample characteristics, in relation to the topic area } \\
\text { being reviewed? }\end{array}$ \\
\hline \multicolumn{2}{|l|}{ (3) ETHICS } \\
\hline Ethics & $\begin{array}{l}\text { 22. Was Ethical Committee approval obtained? } \\
\text { 23. Was informed consent obtained from participants of the study? } \\
\text { 24. Have ethical issues been adequately addressed? }\end{array}$ \\
\hline
\end{tabular}




\begin{tabular}{|c|c|}
\hline \multicolumn{2}{|c|}{ (4) GROUP COMPARABILITY AND OUTCOME MEASUREMENT } \\
\hline $\begin{array}{l}\text { Comparable } \\
\text { Groups }\end{array}$ & $\begin{array}{l}\text { 25. If there was more than one group was analysed, were the groups } \\
\text { comparable before the intervention? In what respects were they } \\
\text { comparable and in what were they not? } \\
26 \text {. How were important confounding variables controlled (e.g. matching, } \\
\text { randomisation, in the analysis stage)? } \\
27 \text {. Was this control adequate to justify the author's conclusions? } \\
\text { 28. Were there other important confounding variables controlled for in } \\
\text { the study design or analyses and what were they? } \\
\text { 29. Did the authors take these into account in their interpretation of the } \\
\text { findings? }\end{array}$ \\
\hline $\begin{array}{l}\text { Outcome } \\
\text { Measurement }\end{array}$ & $\begin{array}{l}\text { 30. What were the outcome criteria? } \\
\text { 31. What outcome measures were used? } \\
\text { 32. Are the measures appropriate, given the outcome criteria? } \\
\text { 33. What other (e.g. process, cost) measures are used? } \\
\text { 34. Are the measures well validated? } \\
\text { 35. Are the measures of known responsive to change? } \\
\text { 36. Whose perspective do the outcome measures address (professional, } \\
\text { service, user, carer)? } \\
\text { 37. Is there a sufficient breath of perspective? } \\
\text { 38. Are the outcome criteria usefulappropriate within routine practice? } \\
\text { 39. Are the outcome measures useful/appropriate within routine } \\
\text { practice? }\end{array}$ \\
\hline $\begin{array}{l}\text { Time Scale of } \\
\text { Measurement }\end{array}$ & $\begin{array}{l}\text { 40. What was the length of follow-up, and at what time points was } \\
\text { outcome measurement made? } \\
\text { 41. Is this period of follow-up sufficient to see the desired effects? }\end{array}$ \\
\hline \multicolumn{2}{|c|}{ (5) POLICY AND PRACTICE IMPLICATIONS } \\
\hline Implications & 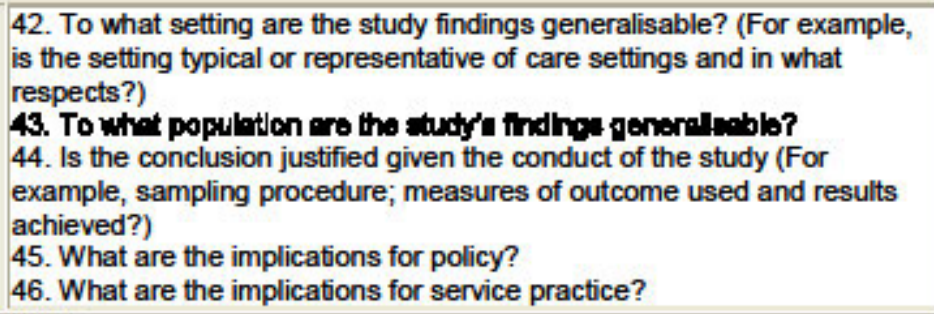 \\
\hline \multicolumn{2}{|c|}{ (6) OTHER COMMENTS } \\
\hline Other Comments & $\begin{array}{l}\text { 47. What were the total number of references used in the study? } \\
\text { 48. Are there any other noteworthy features of the study? } \\
\text { 49. List other study references }\end{array}$ \\
\hline Reviewer & $\begin{array}{l}\text { 50. Name of reviewer } \\
\text { 51. Review date }\end{array}$ \\
\hline
\end{tabular}

Source: Long AF, Godfrey M, Randall T, Brettle AJ and Grant MJ (2002) Developing Evidence Based Social Care Policy and Practice. Part 3: Feasibility of Undertaking Systematic Reviews in Social Care. Leeds: Nuffield Institute for Health.

Note: This tool was developed while the lead author was at the Health Care Practice R\&D Unit (HCPRDU) at the University of Salford. It has since been slightly modified. 


\section{Appendix HH - Mixed Methods Appraisal Tool}

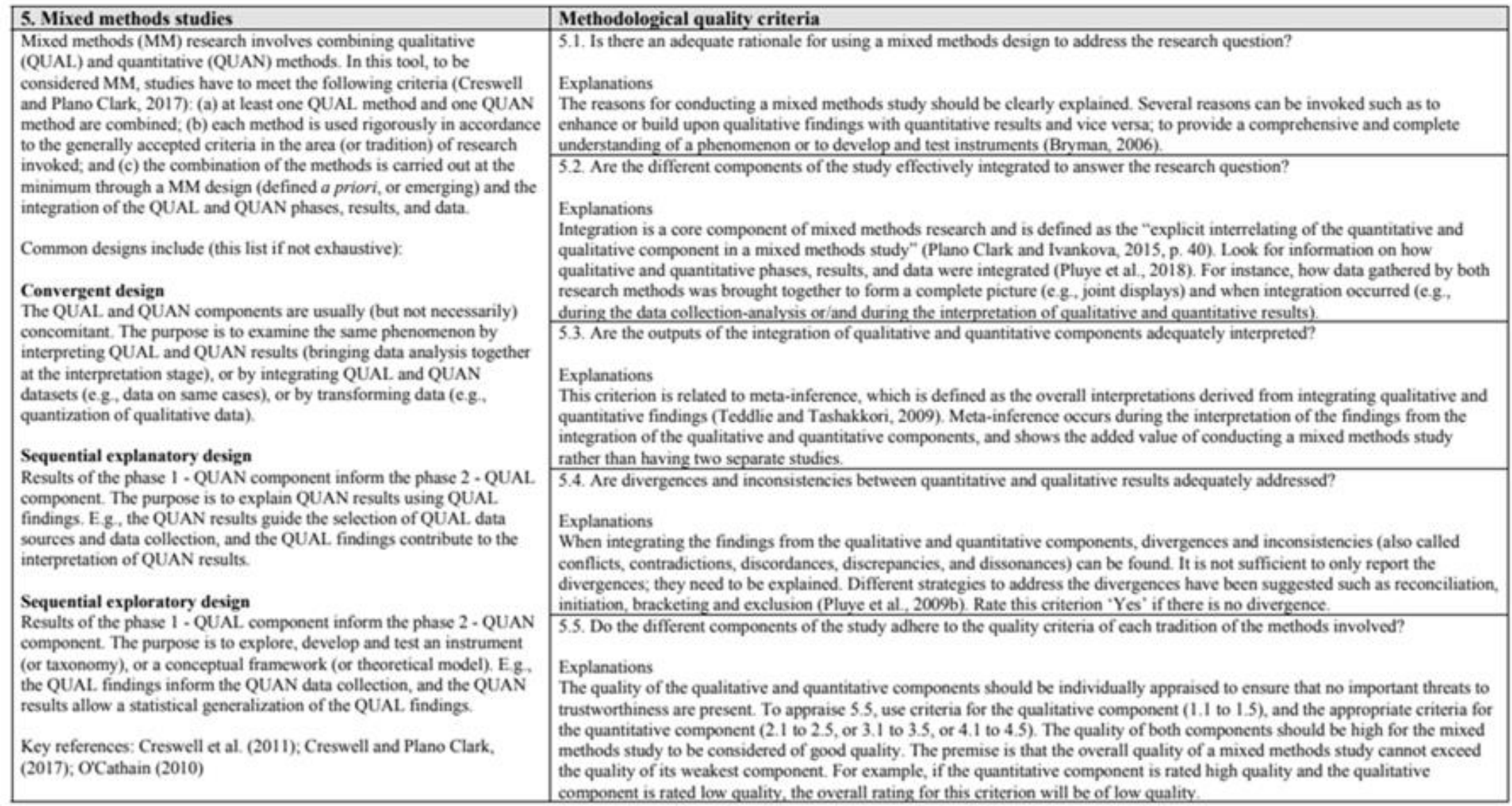



Appendix II - 'If then' statements from the literature review

\section{Staff}

If service-users feel they are treated with care, compassion, dignity and respect (response) then they will form working relationships with staff (outcome). However, if staff are cold, unapproachable and disrespectful (resource) then service-users feel inferior (response), which prevents a relationship from forming (outcome).

If staff are working in inpatient settings where they only see individuals with the greatest needs, or those who are frequently readmitted (context), then they may pessimistic and hopeless towards service-user recovery (outcome).

If staff spend the time to get to know the individual behind the mental illness (resource) then this builds rapport and trust with service-users (response) and develops a therapeutic relationship (outcome). However, staff often have a lack of time to engage in these conversations due to competing priorities (context), leaving service user's feeling alone (response) and with limited opportunities to talk to somebody (outcome).

If staff are unsure of how to use their knowledge of recovery to inform their work and are uncertain about their role in supporting recovery-oriented care (context), then this could limit recovery-oriented care from being implemented within practice (outcome).

If staff are resistant or lack motivation to change to working in recovery-oriented ways (context), then the medical model with remain the dominant framework in inpatient services (outcome).

If staff believe that recovery-oriented care is the responsibility of community and outpatient services (context), then this will create a sense of uncertainty of who is responsibility for recovery-oriented care (response), and will reinforce the medical approach within inpatient settings (outcome).

If there are power imbalances between staff and peer mentors (context), then peer mentors will feel they are not listened to (response), which will be a barrier to their inclusion within services (outcome).

If peer mentors are employed in mental health inpatient settings (resource), then there needs to be wider organisational support through staff understanding the role of peer mentors and how lived experience could be beneficial for service-users (context).

\section{Choice}

If individuals are in crisis, or experience acute mental ill-health (context), then staff assume service-users decision-making abilities have been compromised (response), and therefore limit service-users opportunities for choice and staff make these decisions on their behalf (outcome). It also creates a power imbalance as staff are in a position to remove choice if they deem this relevant (context/outcome). 
If service-users are detained in inpatient settings (context), then they have little choice (outcome) as coercion and compulsory treatment are used (resource). If service-users do not have choice (context), then can recovery-oriented care ever truly be achieved within inpatient settings (outcome).

If service-users decline medical options presented by staff (context), then this was deemed illconsidered by staff (response), preventing the true value of choice from being achieved (outcome).

If service-users are perceptive towards staff's ability to determine the right treatment (context), then they will allow staff to make decisions on their behalf (outcome).

If service-users choose to engage in a particular activity whilst in inpatient care (context), then they have to ask staff for permission (outcome). If staff prioritise meeting individuals needs (context) then they were move likely to support their choices (outcome), whereas if staff prioritise the rules of the ward (context) they are more likely to be risk-adverse in supporting choices (outcome).

If service-users make choices that are harmful, risky or may have bad outcomes (context), then staff are presented with an ethical dilemma between maintaining safety and order, as well as promoting human rights (outcome). As there is a professional responsibility placed upon staff (context), then they may be risk-adverse (response), leading to limited service-user choice (outcome).

If service-users goals and expectations did not align with their professional judgement, then staff were uncertain of how they should support the individual in their choice.

If services experience demands to discharge from external agencies (context), then this may create pressure amongst staff (response) to discharge service-users early (outcome).

If staff make interpretations of service-user needs (context), then this can result in serviceusers requests, questions and needs being dismissed (outcome).

If staff make decisions about service-users care before consulting the service-user (context), then this is not providing recovery-oriented care (outcome).

If there is a no shared language regarding recovery and treatment (context), then this will be a barrier to service-user involvement (outcome), and service-users will be confused (response) by the contradictory advice provided by the clinical team (resource).

\section{Collaboration}

If service-users are in acute distress (context), they may not be able to engage in collaborative care planning (outcome).

If there is a scarcity of time to establish a collaborative relationship (context), then serviceusers may not be involved in decisions about their care (outcome). 
If services assume that carers are provided information by carer groups and advocates (context), then carers feel excluded (response) and a lack of collaboration and information sharing between carers and staff occurs (outcome).

If staff encourage individuals to formulate what they expect from their admission and how the service can help (resource), then service-users can take back agency over their life (outcome).

If individualised approaches to recovery are promoted (resource), then this risks service-users being held responsible and potentially blamed for the success or failure of their recovery (response) and results in a neoliberal view of recovery being adopted in services (outcome).

If service-users lack social skills (context), then their social participation may be impeded (outcome), therefore opportunities to learn and relearn skills would be beneficial (resource).

\section{Community}

If service-users are involved in opportunities outside of the service (resource), then they can identify their social role and form and maintain connections with family, friends and social resources, which will be beneficial upon discharge (outcome).

If staff actively search for opportunities in the community (resource), then this will blur the lines between inpatient settings and the community for both staff and service-users (response). However, these opportunities are typically limited to spaces that welcome those with a mental illness (context), which promotes structural violence and ghettorisation (outcome).

If staff focused upon life outside of hospital (resource), this was scary for some service-users (response), and for those who could not get there basic needs met outside of services (e.g. home, food, money) then there motivation to leave was hindered (outcome).

If there are not sufficient community-based services (resource), then the responsibility of providing support is placed upon carers (response), and service-users may not have access to the resources needed to fulfil their goals and ambitions (outcome). 
- If Woodlands is designed to support individuals who are motivated and ready to engage in their recovery, when policy advocates for least restrictive practice and for the use community-based care, then Woodlands are unlikely to receive the referrals they expected.

- If Woodlands service-user criterion does not align with the current policy direction (e.g. least restrictive practice, community-based care, prudent healthcare, Mental Health (Wales) Measure) then it is likely to continue to receive referrals that the service was not intended to support.

- If Woodlands is categorised as a locked rehabilitation then it will receive referrals based upon this categorisation.

- If Woodlands is classifying itself as providing something unique and different compared to locked rehabilitation services then this difference has not been adequately communicated to commissioners.

- If Woodlands is unable to provide quantifiable evidence of service-user progress (e.g. discharge, employment, symptoms, engagement in community activities) then commissioners will continue to refer to more tried and tested providers.

- If Woodlands does not equip its staff with the training, confidence and skills to support the new service-user group, then they will struggle to be able to fulfil their task of tailoring the service's recovery culture to meet these individual's needs.

- If commissioners are expected to promote least restrictive practice as stated within policy guidelines, then Woodlands' decision to have a locked door may influence the type of individuals referred to the service. 